Helio Aparecido Navarro

\title{
Estudo de Não-linearidades Racionais em Osciladores Mecânicos
}

São Carlos, SP 

Helio Aparecido Navarro

\section{Estudo de Não-linearidades Racionais em Osciladores Mecânicos}

Tese apresentada à Escola de Engenharia de São Carlos da Universidade de São Paulo para a obtenção do título de Professor Livre Docente junto ao Departamento de Engenharia Mecânica.

São Carlos, SP

2017 
AUTORIZO A REPRODUÇÃO E DIVULGAÇÃO TOTAL OU PARCIAL DESTE TRABALHO, POR QUALQUER MEIO CONVENCIONAL OU ELETRÔNICO, PARA FINS DE ESTUDO E PESQUISA, DESDE QUE CITADA A FONTE.

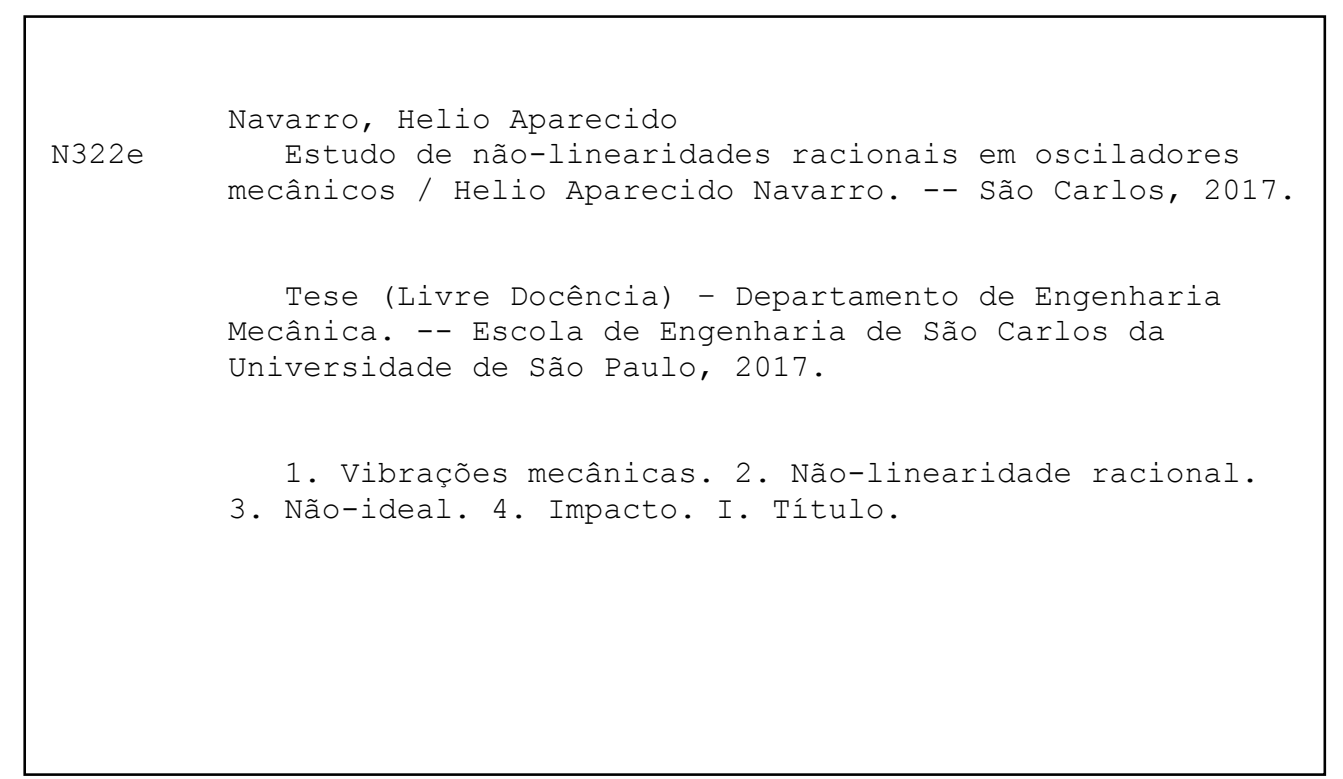


Este trabalho é dedicado:

a minha esposa Eliane, a minha filha Larissa, ao meu filho Gabriel e aos meus pais Helio e Isaura. 



\section{Agradecimentos}

Agradeço a Deus, a minha família, aos meus pais, aos meus familiares, aos meus amigos, aos meus colegas de trabalho do Departamento de Engenharia Mecânica da USP-São Carlos e aos meus colegas da UNESP. 

"Pois nada podemos contra a verdade, só temos poder em favor da verdade."

(2Cor 13, 8) 



\section{Resumo}

NAVARRO, Helio Aparecido. Estudo de Não-linearidades Racionais em Osciladores Mecânicos. 2017. 161f. Tese (Livre Docência) - Escola de Engenharia de São Carlos, Universidade de São Paulo, São Carlos, 2017.

As não-linearidades de ordem fracionária estão presentes em diversos problemas de engenharia envolvendo vibrações mecânicas, acústica, impacto, dispositivos eletro-mecânicos, entre outros. Um tipo de problema especial é o estudo de vibrações em osciladores não-ideais. Geralmente, nesse tipo de problema aparece o efeito Sommerfeld que se caracteriza pela existência de um salto em amplitude e em frequência durante a operação do sistema. No presente trabalho objetiva-se estudar o processo de colisão de osciladores mecânicos nãoideais com não-linearidades de ordem fracionária. As não-linearidades racionais aparecem nos modelos de contato e, também, nas características elásticas dos materiais do oscilador mecânico. Para a delimitação desse problema esta tese apresenta, além da introdução, da revisão bibliográfica e das conclusões, três capítulos principais de desenvolvimento. $\mathrm{O}$ capítulo 3 trata da formulação de soluções aproximadas na forma trigonométrica para problemas de vibração livre em sistemas conservativos com não-linearidades fracionárias. No capítulo 4 são detalhados os modelos de contato baseados na teoria de Hertz. O capítulo 5 apresenta um problema de um oscilador mecânico não-ideal e não-linear colidindo em uma parede rígida. Para o desenvolvimento deste trabalho foram criados diversos códigos computacionais e os resultados obtidos com as soluções aproximadas propostas concordam com os obtidos numericamente. A formulação e a análise realizadas nesta tese constituem uma ferramenta de pesquisa para o desenvolvimento de trabalhos futuros como o estudo do efeito Sommerfeld experimentalmente, entre outros problemas.

Palavras-chave: Vibrações mecânicas. Não-linearidade racional. Não-ideal. Impacto. 



\section{Abstract}

NAVARRO, Helio Aparecido. Study of Rational Nonlinearities in Mechanical Oscillators. 2017. 161f. Thesis - Escola de Engenharia de São Carlos, Universidade de São Paulo, São Carlos, 2017.

The non-linearities of fractional order are present in several engineering problems involving mechanical vibration, acoustics, impact, electro-mechanical devices, among others. A particular type of problem is the study of vibrations in non-ideal oscillators. In this kind of problem it is common to observe the Sommerfeld effect, that is characterized by the existence of a jump in amplitude and frequency during the operation of the system. In the present work we aim to study the collision process of non-ideal mechanical oscillators with non-linearities of fractional order. The rational nonlinearities occur in the contact models and also in the elastic characteristics of the materials of the mechanical oscillator. For the delimitation of this problem, this thesis presents, besides the introduction, the literature review and the conclusions, three main chapters. Chapter 3 deals with the formulation of approximate trigonometric solutions for free vibration problems in conservative systems with rational nonlinearities. In chapter 4 the contact models are detailed based on Hertz's theory. Chapter 5 presents a problem of a non-ideal and non-linear mechanical oscillator colliding in a rigid wall. For the development of this work several computational codes have been created and the results obtained, with the proposed approximate solutions, agree with those obtained numerically. The formulation and the analysis carried out in this thesis constitute a research tool for the development of future works such as the study of the Sommerfeld effect experimentally, among other problems.

Keywords: Mechanical vibration. Rational nonlinearity. Nonideal. Impact. 



\section{Lista de ilustrações}

Figura 1 - Parâmetros $K_{1}(\cdot)$ e $K_{2}(+)$ versus a ordem de não-linearidade $\alpha_{1}$. . . 44

Figura 2 - Solução numérica 'exata', $x_{n}$, (linha contínua) e solução aproximada, $x$, (linha tracejada) para $A=1$ e para $A=0.1\left(c_{1}^{2}=1, c_{2}^{2}=100\right) \ldots . .48$

Figura 3 - Solução numérica 'exata', $x_{n}$, (linha contínua) e solução aproximada, $x$, (linha tracejada) para $A=1$ e para $A=0.1\left(c_{1}^{2}=c_{2}^{2}=c_{3}^{2}=1\right) \ldots$. . . 50

Figura 4 - Erro relativo (\%) versus amplitude, $A$, para $c_{1}^{2}=c_{2}^{2}=c_{3}^{2}=c_{4}^{2}=1$ (linha contínua) e $c_{1}^{2}=c_{2}^{2}=c_{3}^{2}=1, c_{4}^{2}=0$ (linha tracejada). . . . . 5 51

Figura 5 - Erro relativo (\%) versus amplitude, $A$, para $c_{1}^{2}=c_{2}^{2}=c_{3}^{2}=c_{4}^{2}=1$ (linha contínua) e $c_{1}^{2}=c_{4}^{2}=1, c_{2}^{2}=c_{3}^{2}=0$ (linha tracejada). . . . . . 54

Figura 6 - Erros relativos, $\Delta_{T}$ (Eq. 3.116), versus amplitude, $A$, para $v=0$ (linha contínua), $v=10$ (linha tracejada) e $v=100$ (linha pontilhada). . . . . 64

Figura 7 - Erros relativos, $\Delta$ (Eq. 3.121), versus amplitude, $A$, para $v=0$ (linha contínua), $v=10$ (linha tracejada) e $v=100$ (linha pontilhada). . . . 66

Figura 8 - Erros relativos, $\Delta_{T}$ (Eq. 3.116), versus amplitude, $A$, para $v=10$, $\alpha=1 / 2$ (linha contínua), $\alpha=3 / 2$ (Linha tracejada) e $\alpha=3$ (linha pontilhada). . . . . . . . . . . . . . . . . 6 68

Figura 9 - Erros relativo, $\Delta$ (Algoritmo 2), versus amplitude, $A$, para $v=10$, e $\alpha=1 / 2$ (linha contínua), $\alpha=3 / 2$ (linha tracejada) e $\alpha=3$ (linha pontilhada). . . . . . . . . . . . . . . . . .

Figura 10 - Solução numérica, $x(t)$, da Eq. (3.149) (linha contínua) e solução aproximada, $x(t)$, (Eq. 3.156) (linha tracejada): (a) $0 \leq t \leq 60$; (b) $7 \leq t \leq 7.3$. 77

Figura 11 - Solução numérica, $x(t)$, da Eq. (3.149) (linha contínua), solução aproximada, $x(t)$, (Eq. 3.156) (linha tracejada) e solução aproximada, $x(t)$, Eq.(3.157), (linha pontilhada): (a) $0 \leq t \leq 10$; (b) $6.4 \leq t \leq 7.8$. . . . .

Figura 12 - Solução numérica, $x(t)$, da Eq. (3.149) (linha contínua) e solução aproximada, $x(t)$, (Eq. 3.156) (linha tracejada): (a) $0 \leq t \leq 10$; (b) $1 \leq t \leq 5$. 79

Figura 13 - Solução numérica, $x(t)$, da Eq. (3.149) (linha contínua) e solução aproximada, $x(t)$, (Eq. 3.156) (linha tracejada): (a) $0 \leq t \leq 10$; (b) $0.5 \leq t \leq 5.5$. 81

Figura 14 - Esquema de contato entre dois corpos no modelo "esfera-suave" (CUNDALL; STRACK, 1979) . . . . . . . . . . . . . . 84

Figura 15 - Força de contato: sistema massa-mola-amortecedor nas direções normal e tangencial (TSUJI; TANAKA; ISHIDA, 1992) . . . . . . . . . . 85

Figura 16 - Sobreposição adimensional (modelo linear) . . . . . . . . . . . . . . . 90

Figura 17 - Velocidade adimensional (modelo linear) . . . . . . . . . . . . . . . . . 90

Figura 18 - Aceleração adimensional (modelo linear) . . . . . . . . . . . . . . . . . . 91

Figura 19 - Sobreposição adimensional (modelo não-linear, Eq. (4.53)) . . . . . . . . 95 
Figura 20 - Velocidade adimensional (modelo não-linear, Eq. (4.53)) . . . . . . . . . 95

Figura 21 - Aceleração adimensional (modelo não-linear, Eq. (4.53)) . . . . . . . . . 96

Figura 22 - Sobreposição adimensional (modelo não-linear, Eq. (4.71)) . . . . . . . . 99

Figura 23 - Velocidade adimensional (modelo não-linear, Eq. (4.71)) . . . . . . . . . 100

Figura 24 - Aceleração adimensional (modelo não-linear, Eq. (4.71)) . . . . . . . . . 100

Figura 25 - Comparação dos dados experimentias (circulos (STEVENS; HRENYA, 2005)) com os modelos de esfera suave para o coeficiente de restituição (modelo A: linha tracejada; modelo B: linha contínua). . . . . . . . . . 106

Figura 26 - Comparação dos dados experimentias (circulos (STEVENS; HRENYA, 2005)) com os modelos de esfera suave para a duração da colisão (modelo A: linha tracejada; modelo B: linha contínua) . . . . . . . . . . . . . 107

Figura 27 - Esquema da partícula em queda livre. . . . . . . . . . . . . . . . . 111

Figura 28 - Modelo linear $\left(k_{n}=7.94 \times 10^{4} \mathrm{~N} / \mathrm{m}\right.$ e $e_{n}=0.9$ : posição do centro da partícula $y$ (linha sólida); velocidade da partícula $v$ (linha tracejada). . 113

Figura 29 - Modelo linear $\left(k_{n}=7.77 \times 10^{4} \mathrm{~N} / \mathrm{m}\right.$ e $e_{n}=0.7$ : posição do centro da partícula $y$ (linha sólida); velocidade da partícula $v$ (linha tracejada). . 114

Figura 30 - Modelo não-linear "A" $\left(e_{n}=0.9\right)$ : posição do centro da partícula y (linha sólida); velocidade da partícula $v$ (linha tracejada) . . . . . . . . 115

Figura 31 - Modelo não-linear "B" $\left(e_{n}=0.9\right)$ : posição do centro da partícula y (linha sólida); velocidade da partícula $v$ (linha tracejada). . . . . . . 115

Figura 32 - Modelo não-linear "A" $\left(e_{n}=0.7\right)$ : posição do centro da partícula y (linha sólida); velocidade da partícula $v$ (linha tracejada) . . . . . . . 116

Figura 33 - Modelo não-linear "B" $\left(e_{n}=0.7\right)$ : posição do centro da partícula y (linha sólida); velocidade da partícula $v$ (linha tracejada) . . . . . . . 116

Figura 34 - Modelo do sistema mecânico não-ideal. . . . . . . . . . . . . . . . . . . 119

Figura 35 - Curva característica de amplitude e frequência para o sistema e o motor (Adaptado de (ZUKOVIC; CVETICANIN, 2009). . . . . . . . . . . . 128

Figura 36 - Pontos característicos na curva de amplitude e frequência (Adaptado de (CVETICANIN; ZUKOVIC, 2015a). . . . . . . . . . . . . . . 128

Figura 37 - Curva característica de amplitude e frequência para o sistema linear e o motor elétrico $\left(\alpha=1, p^{2}=0.81\right.$ ): (a) $0.6 \leq \Omega \leq 1.3$; (b) $0.89 \leq \Omega \leq 0.93$ (amplificação).

Figura 38 - Curva característica de amplitude e frequência para o sistema linear e o motor elétrico $\left(\alpha=1, p^{2}=1.0\right)$ : (a) $0.6 \leq \Omega \leq 1.3$; (b) $0.99 \leq \Omega \leq 1.04$ (amplificação).

Figura 39 - Histórico no tempo $z-\tau$ para $\alpha=1, p^{2}=0.81, M_{2}=0.92(z(0)=0$, $z^{\prime}(0)=0, \phi(0)=0$ e $\left.\phi^{\prime}(0)=0.0\right)$ : (a) movimento não-estacionário; (b) regime permanente. . . . . . . . . . . . . . . . . 131 
Figura 40 - Histórico no tempo $z-\tau$ para $\alpha=1, p^{2}=0.81, M_{2}=0.92(z(0)=0$, $z^{\prime}(0)=0, \phi(0)=0$ e $\left.\phi^{\prime}(0)=M_{2}\right)$ : (a) movimento não-estacionário; (b) regime permanente. . . . . . . . . . . . . . . . 131

Figura 41 - Histórico no tempo $\Omega-\tau$ para $\alpha=1, p^{2}=0.81, M_{2}=0.92$ : (a) $z(0)=0$, $z^{\prime}(0)=0, \phi(0)=0$ e $\phi^{\prime}(0)=0.0 ;(\mathrm{b}) z(0)=0, z^{\prime}(0)=0, \phi(0)=0$ e $\phi^{\prime}(0)=M_{2}$.

Figura $42-$ Plano de fase $z-z^{\prime}$ para $\alpha=1, p^{2}=0.81, M_{2}=0.92$ : (a) $z^{*}=0.8$, $z(0)=0, z^{\prime}(0)=0, \phi(0)=0$ e $\phi^{\prime}(0)=0.0 ;(b) z^{*}=0.35, z(0)=0$, $z^{\prime}(0)=0, \phi(0)=0$ e $\phi^{\prime}(0)=M_{2}$.

Figura 43 - Curva característica de amplitude e frequência para o sistema não-linear e o motor elétrico $\left(\alpha=4 / 3\right.$ e $\left.p^{2}=0.81\right)$.

Figura 44 - Curva característica de amplitude e frequência para o sistema nãolinear e o motor elétrico $\left(\alpha=5 / 3\right.$ e $p^{2}=0.81$ ): (a) $0 \leq \Omega \leq 1.2$; (b) $0.2 \leq \Omega \leq 0.6$ (amplificação).

Figura 45 - Histórico no tempo para $\alpha=4 / 3, M_{2}=0.8, p^{2}=0.81,(z(0)=0$, $z^{\prime}(0)=0, \phi(0)=0$ e $\left.\phi^{\prime}(0)=0.0\right):($ a) $z-\tau ;$ (b) $\Omega-\tau \ldots \ldots$. . . .

Figura 46 - Histórico no tempo para $\alpha=4 / 3, M_{2}=0.8, p^{2}=0.81,(z(0)=0$,

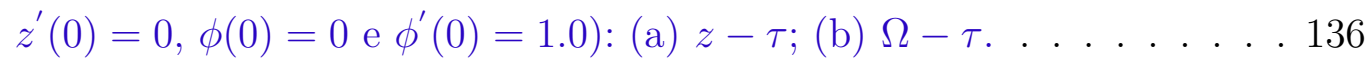

Figura 47 - Histórico no tempo para $\alpha=5 / 3, M_{2}=0.7, p^{2}=0.81,(z(0)=0$, $z^{\prime}(0)=0, \phi(0)=0$ e $\left.\phi^{\prime}(0)=0.0\right):($ a) $z-\tau ;($ b) $\Omega-\tau$.

Figura 48 - Histórico no tempo para $\alpha=5 / 3, M_{2}=0.7, p^{2}=0.81,(z(0)=0$, $z^{\prime}(0)=0, \phi(0)=0$ e $\left.\phi^{\prime}(0)=1.0\right):($ a $) z-\tau ;($ b) $\Omega-\tau$.

Figura 49 - Curva característica de amplitude e frequência para o sistema não-linear e o motor elétrico $\left(\alpha=2 / 3\right.$ e $\left.p^{2}=0.36\right)$.

Figura 50 - Diagramas de impacto, $\alpha=4 / 3, p^{2}=0.81, M_{2}=0.8, z^{*}=0.4, z(0)=0$, $z^{\prime}(0)=0, \phi(0)=0$ e $\phi^{\prime}(0)=0.0$ : (a) Histórico no tempo $z-\tau$; (b) Plano de fase $z-z^{\prime}$.

Figura 51 - Diagramas de impacto, $\alpha=4 / 3, p^{2}=0.81, M_{2}=0.8, z^{*}=0.018$, $z(0)=0, z^{\prime}(0)=0, \phi(0)=0$ e $\phi^{\prime}(0)=1.0$ : (a) Histórico no tempo $z-\tau$; (b) Plano de fase $z-z^{\prime}$.

Figura 52 - Diagramas para $V=0.336 \mathrm{~V}$ : (a) retrato de fase; (b) histórico no tempo $\phi-t$; (c) histórico no tempo $\dot{\phi}-t \ldots \ldots$. . . . . . . . . . 142

Figura 53 - Diagramas para $V=24.0 \mathrm{~V}$ : (a) retrato de fase; (b) histórico no tempo $\phi-t$; (c) histórico no tempo $\dot{\phi}-t . \ldots . \ldots . \ldots 143$

Figura 54 - Curvas de amplitude-frequência: (a) $5 . .50 \mathrm{rad} / \mathrm{s}$; (b) $7 . .15 \mathrm{rad} / \mathrm{s}$ (amplificação). 143

Figura 55 - Curvas de histórico no tempo $(V=0.336 \mathrm{~V})$ : (a) $0 \leq t \leq 600 \mathrm{~s}$; (b) $0 \leq t \leq 10 \mathrm{~s}$ 
Figura 56 - Diagramas de planos de fase: (a) $V=0.336 \mathrm{~V}$; (b) $V=0.336 \mathrm{~V}$ (amplificação). . . . . . . . . . . . . . . . . . . 145

Figura 57 - Curva de amplitude-frequência (asteriscos - para aumento de $V$; Círculos

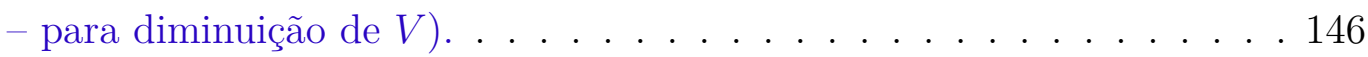




\section{Lista de tabelas}

Tabela 1 - Valores dos parâmetros $K_{1}, K_{2}$ e erros relativos (Eq. 3.56) para valores

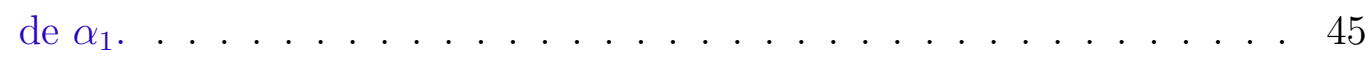

Tabela 2 - Valores dos erros relativos para diversas ordens de não-linearidade $\alpha_{1}$. 47

Tabela 3 - Período de vibração e erros relativos para diversos métodos aproximados (adaptado de Cveticanin (2009a, página 1358)). . . . . . . . . . . 49

Tabela 4 - Período numérico, período aproximado e erros relativos para diversos conjuntos dos parâmetros $c_{1}^{2}, c_{2}^{2}, c_{3}^{2}$, e $c_{4}^{2}$ e valor de amplitude inicial

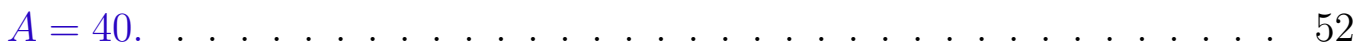

Tabela 5 - Erro relativo para $c_{1}^{2}=c_{2}^{2}=c_{3}^{2}=c_{4}^{2}=1$ e diversas amplitudes inicais $A .55$

Tabela 6 - Erro relativo para $c_{1}^{2}=c_{4}^{2}=1, c_{2}^{2}=c_{3}^{2}=0$ e diversas amplitudes iniciais A. . . . . . . . . . . . . . . . . 56

Tabela 7 - Erros relativos, $\Delta_{T}$ (Eq. 3.116), para $c_{1}^{2}=c_{2}^{2}=1$ e diversas condições iniciais $A, v \ldots \ldots \ldots \ldots$. . . . . . . . . . . . . . . . . . . . .

Tabela 8 - Valores dos erros relativos, $\Delta_{T}(A, v)$ e $\Delta(A, v)$, para diversos valores

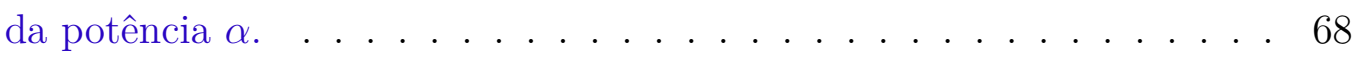

Tabela 9 - Parâmetros adimensionais para o modelo linear. . . . . . . . . . . . . 91

Tabela 10 - Parâmetros adimensionais para o modelo não-linear (4.53) . . . . . . . . 96

Tabela 11 - Parâmetros adimensionais para o modelo não-linear, Eq. (4.71). . . . 101

Tabela 12 - Comparação dos valores de $\nu_{B}^{*}$ para diversos modelos da força dissipativa.104

Tabela 13 - Duração da colisão, $t_{c, n}^{*}$, para o modelo não-linear , Eq. (4.71) . . . . . . 105

Tabela 14 - Coeficiente de rigidez normal para o modelo linear: colisão binária. . 109

Tabela 15 - Coeficiente de rigidez normal para o modelo linear: colisão com a parede.110

Tabela 16 - Parâmetros de simulação para a partícula em queda livre. . . . . . . . . 111

Tabela 17 - Coeficiente de rigidez normal para o modelo linear: partícula em queda livre. . . . . . . . . . . . . . . . . . . . . . 112

Tabela 18 - Parâmetros do sistema mecânico para a simulação numérica. . . . . . . 141

Tabela 19 - Parâmetros de Colisão. . . . . . . . . . . . . . . . . . . . . . . 142 



\section{Lista de abreviaturas e siglas}

DEM Discrete Element Method (Método dos Elementos Discretos)

MFIX Multiphase Flow with Interphase eXchanges 



\section{Lista de símbolos}

\begin{tabular}{|c|c|}
\hline$A$ & Condição inicial para a posição, $[\mathrm{m}]$ \\
\hline$c$ & Coeficiente de amortecimento, $[\mathrm{Ns} / \mathrm{m}]$ \\
\hline$c_{k}$ & Coeficientes \\
\hline$e$ & Coeficiente de restituição \\
\hline$E$ & Módulo de Young, $[\mathrm{Pa}]$ \\
\hline$F$ & Força, $[\mathrm{N}]$ \\
\hline$F_{I}$ & Força de impacto, $[\mathrm{N}]$ \\
\hline$g$ & Aceleração da gravidade, $\left[\mathrm{m} / \mathrm{s}^{2}\right]$ \\
\hline$G$ & Distância, $[\mathrm{m}]$ \\
\hline$H$ & Hamiltoniano, $[\mathrm{J}]$ \\
\hline$J$ & Momento de inércia de massa, $\left[\mathrm{kgm}^{2}\right]$ \\
\hline$k$ & Rigidez, $\left[\mathrm{N} / \mathrm{m}^{\alpha}\right]$ \\
\hline$K$ & Energia Cinética, $[\mathrm{J}]$ \\
\hline$K_{E}$ & Constante de voltagem, $\left[\mathrm{V} / \mathrm{rads}^{-1}\right]$ \\
\hline$K_{T}$ & Constante de torque, $[\mathrm{Nm} / \mathrm{A}]$ \\
\hline$L$ & Grau de polinômio \\
\hline$M$ & Grau de polinômio \\
\hline$M, m$ & Massa, $[\mathrm{kg}]$ \\
\hline$M_{m}$ & Torque do motor, $[\mathrm{Nm}]$ \\
\hline$M_{0}, M_{1}, M_{2}$ & Torque do motor, $[\mathrm{Nm}]$ \\
\hline$Q$ & Força generalizada, $[\mathrm{N}]$ \\
\hline$r$ & Raio, $[\mathrm{m}]$ \\
\hline$R$ & Resistência elétrica, $[\Omega]$ \\
\hline
\end{tabular}




$\begin{array}{ll}t & \text { Instante, }[\mathrm{s}] \\ T & \text { Período, }[\mathrm{s}] \\ T & \text { Energia Cinética, }[\mathrm{J}] \\ V & \text { Energia Potencial, }[\mathrm{J}] \\ v & \text { Velocidade, }[\mathrm{m} / \mathrm{s}] \\ x & \text { Posição, }[\mathrm{m}] \\ X & \text { Coeficiente da Série de Taylor } \\ z & \text { Posição, }[\mathrm{m}]\end{array}$

\section{LETRAS GREGAS}

$\begin{array}{ll}\alpha & \text { Ordem de não-linearidade } \\ \beta & \text { Ordem de não-linearidade } \\ \delta & \text { Sopreposição, }[\mathrm{m}] \\ \Delta & \text { Erro } \\ \eta & \text { Coeficiente de amortecimento, }\left[\mathrm{Ns} / \mathrm{m}^{\mathrm{p}+1}\right] \\ \Gamma & \text { Função Gamma } \\ \nu^{*} & \text { Coeficiente de amortecimento adimensional } \\ \omega & \text { Frequência, }[\mathrm{rad} / \mathrm{s}] \\ \Omega & \text { Rotação, }[\mathrm{rad} / \mathrm{s}] \\ \phi & \text { Posição angular } \\ \Phi & \text { Função dissipativa, }[\mathrm{Nm} / \mathrm{s}] \\ \rho & \text { Massa específica, }\left[\mathrm{kg} / \mathrm{m}^{3}\right] \\ \sigma & \text { Coeficiente de Poisson }\end{array}$




\section{Sumário}

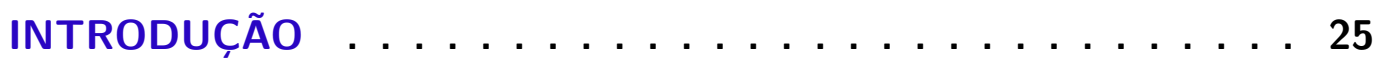

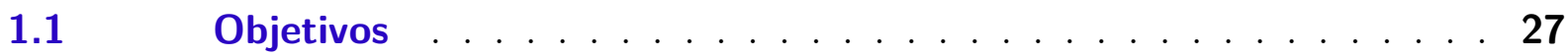

$1.2 \quad$ Organização da tese $\ldots \ldots \ldots \ldots \ldots \ldots \ldots$

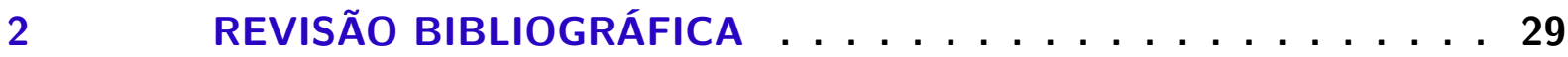

3 VIBRAÇÕES MECÂNICAS NÃO-LINEARES . . . . . . . . 35

3.1 Vibração livre de osciladores conservativos com condições iniciais

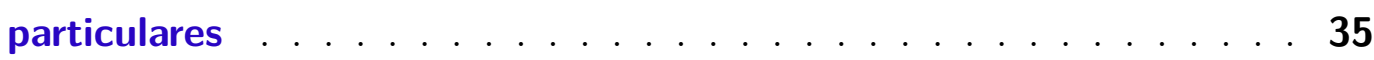

3.1 .1 Solução aproximada . . . . . . . . . . . . . . . . . 36

3.1.2 Estimativa de erro da solução . . . . . . . . . . . . . . . . 40

3.1.3 Comparação entre as soluções aproximada e exata . . . . . . . . . . . . 41

3.1.3.1 Equação com um termo conservativo . . . . . . . . . . . . . . . . . . . . 41

3.1.3.2 Equação com diversos termos conservativos . . . . . . . . . . . . . . . . 47

3.2 Vibração livre de osciladores conservativos com condições iniciais

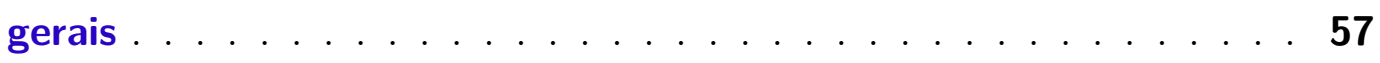

3.2.1 Solução aproximada . . . . . . . . . . . . . . . . . . . 57

3.2.2 Estimativa de erro da solução . . . . . . . . . . . . . . . . 60

3.2.3 Comparação entre as soluções aproximadas e numéricas . . . . . . . . . . . 61

3.2.3.1 Equação de Duffing . . . . . . . . . . . . . . . . . 62

3.2.3.2 Oscilador conservativo não-linear puro . . . . . . . . . . . . 66

3.3 Vibração livre de osciladores com amortecimento linear . . . . . 70

3.3.1 Exemplo de oscilador conservativo . . . . . . . . . . . . . . . 75

3.3.2 Exemplos de osciladores não-conservativos . . . . . . . . . . . . . 77

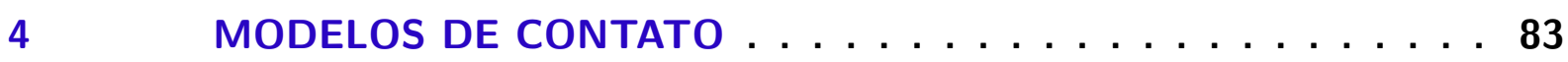

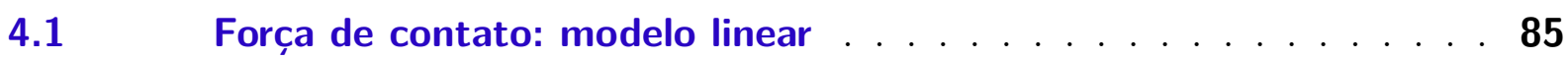

$4.2 \quad$ Força de contato: modelo não-linear . . . . . . . . . . . . 92

$4.3 \quad$ Coeficiente de rigidez - modelo linear . . . . . . . . . . . 106

$4.4 \quad$ Partícula em queda livre . . . . . . . . . . . . . . . . 111

4.4.1 Modelo linear . . . . . . . . . . . . . . . . . . . . . . . . . . . 112

4.4 .2 Modelo não-linear . . . . . . . . . . . . . . . . . . . . . . . . . . . . . 114

5 SISTEMAS COM FONTE DE POTÊNCIA LIMITADA . . . . . 117

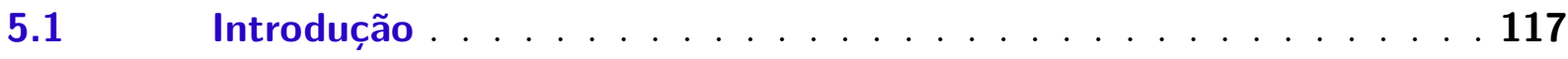

5.2 Modelo do sistema mecânico não-ideal . . . . . . . . . . . . 118 
5.3 Exemplos numéricos de um sistema mecânico não-ideal . . . . . . 129

5.3.1 Sistema mecânico linear . . . . . . . . . . . . . . . . . . . . . . . . . . 129

5.3.2 Sistema mecânico não-linear . . . . . . . . . . . . . . . . . . . . 133

5.3.3 Sistema mecânico colidindo em um parede com forças dissipativas . . . . . 140

6 CONCLUSÕES E RECOMENDAÇÕES . . . . . . . . . . 147

REFERÊNCIAS . . . . . . . . . . . . . . 151 


\section{Introdução}

Na engenharia existem diversas aplicações em que a dinâmica linear e não-linear estão presentes, por exemplo, projetos de aviões, turbinas, pontes, automóveis, entre outras. No entanto, para que essas aplicações sejam eficientes durante seu funcionamento devem ser estudadas as características dos sistemas dinâmicos, buscando as instabilidades e as oscilações que podem ocorrer. Esses sistemas podem ser classificados como ideais e não ideais, de acordo com a forma de excitação dos mesmos.

Os sistemas ideais são caracterizados por ter uma fonte externa com potência ilimitada, em que a excitação externa é integrada juntamente com as equações de movimento da estrutura. Os sistemas não-ideais são caracterizados pela influência da estrutura na fonte de excitação e vice-versa. Para a solução de sistemas não-ideais deve-se adicionar uma equação que descreva o comportamento da fonte não-ideal (ver (KONONENKO, 1969)). Essas fontes têm potência limitada com curvas características como, por exemplo, os motores elétricos de corrente contínua. A primeira descrição da interação entre um sistema oscilante e a fonte de energia foi feita por Sommerfeld (1902). Em seu experimento, utilizando uma mesa acoplada a um motor elétrico, A. Sommerfeld observou que a velocidade do motor não dependia apenas da energia inserida no sistema. Foi verificado que a amplitude de oscilação atinge o valor máximo na região de ressonância, ponto em que o consumo de energia cresce. Após a ressonância, a amplitude decresce bruscamente, enquanto a velocidade do motor cresce rapidamente, aparecendo um salto em amplitude e em frequência. Um exemplo de sistema não-ideal que se destaca por sua aplicabilidade é o oscilador não-ideal simples com massa, mola, amortecedor e fonte de potência limitada, com aplicações em veículos, estruturas acopladas a motores elétricos, sistemas pneumáticos, elétricos, fluídicos, entre outras aplicações.

Os modelos matemáticos dos sistemas dinâmicos não-lineares podem ser resolvidos analiticamente ou numericamente. No caso numérico, a solução fornece informações qualitativas e quantitativas dos fenômenos físicos que podem ocorrer devido ao aparecimento de não-linearidades, por exemplo, saltos, ressonâncias, interações modais, caos, entre outras. No caso analítico, quando a solução exata não é possível, utiliza-se a teoria das perturbações para a obtenção de soluções aproximadas para valores pequenos dos parâmetros. A teoria das perturbações tradicional fornece uma ideia geral da característica das soluções dos problemas. Existem outros métodos recentes, geralmente baseados em técnicas assintóticas, que são válidos, não somente para sistemas levemente não-lineares, mas também para os fortemente não-lineares, sendo que as soluções analíticas aproximadas obtidas são válidas para grande parte ou todo domínio. 
Esses métodos, diferentemente dos primeiros, da teoria de pertubação tradicional, são denominados de métodos perturbativos modificados ou não-perturbativos. Diversos métodos foram desenvolvidos para osciladores levemente não-lineares (ver (NAYFEH, 1981)), como os métodos das múltiplas escalas, os métodos das médias, os métodos baseados em expansão assintótica, entre outros. Recentemente, um número significativo de métodos que foram desenvolvidos para osciladores levemente não-lineares estão sendo adotados para a solução de osciladores fortemente não-lineares, tais como: método de Lindstedt-Poincare (AMORE; ARANDA, 2005), método do balanço harmônico (MICKENS, 1984; HE, 2002), método da pertubação homotópica (HE, 1999a; HE, 2003), método de interação variacional (HE, 1999b), abordagem Hamiltoniana (HE, 2010; BAYAT; PAKAR; CVETICANIN, 2014), método de Krylov-Bogolubov (NAYFEH, 2004; BAYAT; PAKAR, 2013), entre outros.

Em 2010, um método denominado de abordagem Hamiltoniana foi derivado para resolver sistemas oscilatórios altamente não-lineares (HE, 2010). Desde essa época, diversos artigos foram publicados estendendo e aplicando o referido método. Nos artigos de Akbarzade e Kargar (2011a) e Akbarzade e Kargar (2011b) o método é aplicado para a obtenção de soluções analíticas precisas para osciladores não-lineares. Usando a abordagem Hamiltoniana He, Zhong e L. (2010) e Bayat, Pakar e Cveticanin (2014) obtiveram a solução para a equação de Duffing, enquanto Cveticanin et al. (2010) e Cveticanin, Kalami-Yazdi e Askari (2012) obtiveram uma solução generalizando-a para osciladores não-lineares com potência fracionária. Yildirim, Saadatnia e Askari (2011) e Belendez et al. (2011) especificaram o método para osciladores com termos elásticos racionais e irracionais. Xu e He (2010) determinaram o movimento de ciclo limite usando a abordagem Hamiltoniana. Esse método também é aplicado para resolver problemas práticos, como, osciladores não-lineares de uma carga localizada em um campo elétrico em um anel carregado (YILDIRIM et al., 2011), vibração não-linear em sistemas mecânicos micro-elétricos (SADEGHZADEH; KABIRI, 2016), oscilações não-lineares em estruturas (AKBARZADE; KHAN, 2012), entre outras aplicações. Para melhorar a precisão da solução diversos autores desenvolveram a abordagem Hamiltoniana com soluções aproximadas de alta ordem para osciladores não-lineares (DURMAZ; DEMIRBAG; KAYA, 2010; YILDIRIM et al., 2011), com múltiplos osciladores acoplados (DURMAZ; DEMIRBAG; KAYA, 2012) e com múltiplos parâmetros (KHAN; JAMIL; ARA, 2011). Nos métodos listados anteriormente a precisão da solução é comprovada com a solução obtida numericamente. Entretanto, em muitos casos, as diferenças quantitativas ente as soluções não é pequena para grandes tempos de simulação.

Mickens (2001) apresentou aplicações de osiladores com não-linearidade de determinada ordem não-inteira. Seguindo esse autor, diversos trabalhos foram investigados na direção de generalização do modelo em que a não-linearidade possuía qualquer número racional (inteiro ou não-inteiro), tais como: Cveticanin et al. (2010), Cveticanin, Kovacic e 
Rakaric (2010), Cveticanin, Kalami-Yazdi e Askari (2012), Herişanu e Marinca (2010), Kovacic, Rakaric e Cveticanin (2010), Cveticanin (2014), Cveticanin e Pogany (2012), entre outros.

A solução de sistemas dinâmicos na presença de impacto pode levar a configurações caóticas do sistema. Uma abordagem do tratamento do contato advém de escoamentos granulares na interação entre partícula-partícula e partícula-parede. Alder e Wainwright (1957) introduziram métodos da dinâmica molecular como uma metodologia para estudar o comportamento macroscópico de partículas. As técnicas desenvolvidas para a dinâmica molecular podem ser adaptadas para modelos de partículas discretas, incluindo na formulação as interações entre partículas-partículas. Cundall e Strack (1979) propuseram um modelo derivado da dinâmica molecular combinando-o com mecanismos de contato. Esse modelo é denominado de Método dos Elementos Discretos ("Discrete Element Method" - DEM) e tem sido aplicado em diversas áreas, como em mecanismos geotécnicos (CUNDALL; STRACK, 1979); tecnologias de transporte pneumáticos (TSUJI; TANAKA; ISHIDA, 1992); leitos fluidizados (TSUJI; KAWAGUCHI; TANAKA, 1993); segregação de materiais granulares (KETTERHAGEN et al., 2007); escoamento de partículas coesivas (WEBER, 2004); entre outros. Uma revisão abrangente da literatura sobre o método DEM é apresentada nos trabalhos de Zhu et al. (2007) e Zhu et al. (2008). O livro de Ibrahim (2009) apresenta um estudo detalhado da dinâmica de vibro-impacto sob os aspectos de modelagem e aplicações.

Feitas as considerações iniciais de introdução a esta tese, nos próximos itens deste capítulo são descritos os objetivos e a estrutura deste trabalho.

\subsection{Objetivos}

O objetivo principal desta tese é estudar a colisão de osciladores mecânicos nãoideais com não-linearidade de ordem fracionária. Assim, para o desenvolvimento do presente trabalho são definidos os seguintes objetivos específicos:

- formular soluções aproximadas na forma trigonométrica para problemas de vibração livre em sistemas conservativos com não-linearidades racionais e em sistemas não-conservativos (amortecimento linear). Esse estudo é realizado no Capítulo 3. Em problemas mais complexos (por exemplo, osciladores mecânicos não-ideias com nãolinearidade de ordem fracionária), em que se têm parâmetros pequenos, pode-se escrever soluções aproximadas na forma trigonométrica para a construção de gráficos das curvas características de amplitude-frequência do sistema;

- detalhar os modelos de contato utilizando a abordagem DEM em que os cálculos dos parâmetros elásticos são baseados nas propriedades dos materiais (modelos Hertzianos, ver (HERTZ, 1882)) e que as forças não-conservativas são especificadas por modelos 
contínuos (HUNT; GROSSLEY, 1975) (ver Capítulo 4). Baseado na abordagem DEM, durante o impacto, o sistema mecânico é modelado acoplando-o a uma mola e a um amortecedor, sendo que os coeficientes desses elementos têm não-linearidades de ordem não-inteira;

- solucionar analiticamente e numericamente os problemas não-ideias com impacto. Cveticanin e Zukovic (2015a) derivaram equações para problemas em que os osciladores mecânicos eram fortemente não-lineares com qualquer ordem de não-linearidade (inteira ou não-inteira). Utilizando os métodos descritos nos Capítulos 3 e 4, objetiva-se complementar o trabalho de Cveticanin e Zukovic (2015a), considerando um sistema mecânico não-ideal com não-linearidades racionais impactando em uma parede rígida, ver Capítulo 5.

\subsection{Organização da tese}

Esta tese está organizada em seis capítulos. O primeiro capítulo apresenta a introdução da tese e contém uma contextualização bibliográfica sobre sistemas mecânicos com não-linearidades racionais, sistemas não-ideais e modelos de contato. Em seguida, são listados o objetivo principal da tese e os estudos a serem realizados para se alcançar esse objetivo. O capítulo 2 traz a revisão bibliográfica, discorrendo sobre trabalhos da literatura que tratam de temas de pesquisa correlatos a esta tese. O capítulo 3 apresenta o estudo de vibrações mecânicas não-lineares. Nesse capítulo obtém-se soluções aproximadas e numéricas da vibração livre de osciladores conservativos e não-conservativos com nãolinearidade de ordem inteira e não-inteira. A abordagem dos elementos discretos e a descrição de modelos de contato linear e não-lineares são detalhadas capítulo 4 . O capítulo 5 apresenta uma análise analítica e numérica de sistemas mecânicos com impacto excitados por uma fonte de potência limitada. O capítulo 6 contém as conclusões e recomendações para trabalhos futuros. As referências bibliográficas estão listadas na sequência do capítulo 6. 


\section{Revisão Bibliográfica}

Este capítulo apresenta a contextualização bibliográfica com trabalhos que tratam do tema desta tese. A dinâmica não-linear tem sido estudada por diversos autores e faz parte de sistemas oscilatórios presentes na natureza, como nos fenômenos meteorológicos, nos biológicos, entre outros fenômenos e, também, está presente nas indústrias, nos equipamentos ou dispositivos atuais, como máquinas rotativas, micro-atuadores, estruturas de concreto, reatores, entre outros equipamentos. Alguns textos tradicionais que tratam de vibrações não-lineares são os seguintes: Kononenko (1969) descreve os sistemas acoplados com fonte de potência limitada, Nayfeh e Mook (1976) descrevem sistemas não-lineares com um e número finito de graus de liberdade, sistemas excitados parametricamente e sistemas contínuos, Krylov e Bogoliubov (1947) apresentam alguns osciladores não-lineares e soluções aproximadas baseadas em linearização, em periodicidade do sistema, entre outras abordagens, Cunningham (1958) trata de soluções aproximadas de equações nãolineares, da estabilidade de sistemas não-lineares e, também, contém um capítulo dedicado à derivação de soluções exatas de determinadas equações não-lineares, como por exemplo um oscilador mecânico conectado com uma mola não-linear, Fidlin (2006) descreve diversos modelos e soluções aproximadas de problemas não-lineares, como, sistemas oscilatórios com atrito seco, sistemas com colisão quase elástica, sistemas com grande dissipação devido ao alto amortecimento durante colisões, sistemas com alta frequência de excitação, entre outros, Timoshenko (1937) é um livro clássico de vibração que tem um capítulo que trata de vibração de sistemas com características não-lineares, entre outros livros.

As não-linearidades em sistemas mecânicos são comuns e podem decorrer dos tipos de materiais, das características geométricas, das grandes deformações, entre outros fatores. A dinâmica não-linear tem por objetivo avaliar sistemas dinâmicos complexos, com respostas periódicas, multiperiódicas, quasiperiódicas e caóticas. Um exemplo de sistema não-linear que se destaca por sua aplicabilidade é o oscilador não-ideal simples (KONONENKO, 1969) com uma fonte de potência limitada, massa, mola e amortecedor, com aplicações em veículos, estruturas acopladas a motores elétricos, sistemas pneumáticos, elétricos, fluídicos, entre outras aplicações.

Existem diversos sistemas mecânicos que têm propriedades matemáticas de descontinuidades. Esses sistemas são denominados de não-suaves, como por exemplo, sistemas mecânicos que apresentam atrito seco, impacto, folga, entre outros. Os sistemas dinâmicos não-suaves aparecem nos estudos de fenômenos físicos, biológicos, mecânicos, entre outros processos. O livro de Ibrahim (2009) detalha a dinâmica de vibro-impacto de sistemas mecânicos não-suaves. A solução de sistemas dinâmicos na presença de impacto pode levar a configurações caóticas do sistema. 
Uma abordagem para o tratamento das forças de contato advém de escoamentos granulares na interação do particulado. Alder e Wainwright (1957) introduziram métodos da dinâmica molecular como uma metodologia para estudar o comportamento macroscópico de partículas. Essa técnica em conjunto com os mecanismos de contato definem um modelo de Cundall e Strack (1979) denominado de Método dos Elementos Discretos (DEM). Esse método é aplicado em diversas áreas, como na simulação de escoamentos granulares em que cada partícula é analisada individualmente. Dois modelos são usados na modelagem de corpos (ou partículas) quando se utiliza o DEM: o modelo de esfera rígida e o modelo de esfera suave. O modelo de esfera rígida é usado em regime do particulado diluído, isto é, quando as partículas estão dispersas e as colisões entre partículas é análoga a dinâmica molecular em gases rarefeitos. A troca de energia no sistema ocorre por meio das colisões das partículas, da aceleração das partículas, da gravidade e pelas colisões com a fronteira. $\mathrm{Na}$ área de escoamento granulares, a maioria dos trabalhos publicados na literatura tem considerado apenas colisões binárias. As partículas se movimentam em uma trajetória bem definida até que a colisão com outra partícula ocorra. Essa interação entre as partículas são modeladas como colisões instantâneas e o estado de pós-colisão é determinado a partir da dinâmica clássica (CAMPBELL, 1982). O modelo de esfera rígida considera as equações Newtonianas de movimento na forma integral e é baseado nas forças impulsivas e repulsivas que são definidas pelas forças atuando nas partículas durante a colisão. As equações e a descrição detalhada do modelo de esfera rígida podem ser encontradas em Campbell (1982) e Crowe, Sommerfeld e Tsuji (1997). Diversos autores utilizaram o modelo esfera rígida, tais como, Hoomans et al. (1996) usaram esse modelo em leitos fluidizados para analisar o comportamento das partículas na formação de bolhas e durante o processo de segregação. Goldschmidt, Beetstra e Kuipers (2004) utilizaram leitos fluidizados para fazer uma comparação entre o modelo de esfera rígida, o modelo contínuo e testes experimentais, mostrando que os resultados se aproximam dos dados experimentais. Lu et al. (2005) investigaram, também, o comportamento das partículas em um leito fluidizado usando o modelo de esfera rígida. Os autores concluíram que o modelo esfera rígida pode fornecer informações detalhadas das configurações do particulado, no entanto, requer um elevado tempo computacional. De acordo com Müller e Pöschel (2012) existem limitações na utilização do modelo esfera rígida, dependendo do tipo de material e dos parâmetros do sistema. Wang, He e Kim (2015) utilizaram o modelo de esfera rígida para investigar o comportamento de um leito fluidizado borbulhante sob o efeito de diferentes valores da aceleração da gravidade. Os resultados indicaram que os comportamentos das bolhas são significativamente alterados pelas mudanças na gravidade.

Uma grande parte dos escoamentos granulares e gás-sólidos apresenta um regime denso (atrito), em que as partículas estão em contato uma com as outras por um longo período de tempo. O modelo da esfera suave, proposto por Cundall e Strack (1979), é o mais recomendável para ser utilizado na modelagem desse regime. Nesse modelo, as 
partículas são permitidas se "sobreporem (overlap)" em uma pequena distância e as forças de contato são calculadas com base no histórico de deformação usando um mapeamento entre essas forças e um sistema dinâmico. Um estudo detalhado sobre o processo de impacto é apresentado no livro de Goldsmith (1960). O autor mostra o mecanismo de contato e deriva relações entre força e deformação como a lei de contato de Hertz (HERTZ, 1882). Goldsmith (1960) descreve experimentos envolvendo impacto entre esferas, esferas e placas, barras, vigas, entre outros elementos, e também determina a dinâmica das propriedades de diversos materiais sujeitos a tração, compressão, flexão e torção. Timoshenko e Goodier (1970) fornecem uma apresentação clássica da teoria da elasticidade. A energia perdida durante o impacto é associada com mecanismos de amortecimento durante o período de contato. Simo e Hughes (1997) apresentam conceitos teóricos de inelasticidade, uma formulação numérica e, também, uma descrição computacional de algoritmos clássicos de modelos de plasticidade, viscoplasticidade e viscoelastidade. Diversos esquemas têm sido propostos na literatura para modelar as forças de contato, como o de Walton e Braun (1986) que usam duas constantes elásticas diferentes para modelar a energia de dissipação nas direções normal e tangencial. Hunt e Grossley (1975) discutem o modelo de Kelvi-Voigt e introduzem um novo termo de amortecimento não-linear para modelar o impacto entre os corpos sólidos. Yigit, Ulsoy e Scott (1990) comparam, com dados experimentais de vigas flexíveis, alguns modelos do tipo massa mola e amortecedor para caracterizar o impacto. Em escoamentos gás-sólido, o modelo de esfera suave possibilita a análise de algumas interações entre as partículas e entre partículas e parede, que não podem ser obtidas no modelo de esfera rígida.

O modelo dinâmico linear massa-mola-amortecedor é amplamente utilizado nas simulações DEM para o cálculo das forças de contato (ver, por exemplo, (HAFF; WERNER, 1986; HOEF et al., 2006; GARG et al., 2010; BENYAHIA; GALVIN, 2010; GOPALAKRISHNAN; TAFTI, 2013)). Alguns motivos para isso são que o modelo linear é simples, tem solução analítica para os parâmetros de colisão e é computacionalmente mais econômico comparado com os modelos não-lineares. No modelo linear é necessário especificar os valores dos coeficientes de rigidez e de amortecimento. Shäfer, Dippel e Wolf (1996) descrevem que o modelo linear não tem parâmetros livres desde que os coeficientes de rigidez e de amortecimento podem ser ajustados com o coeficiente de restituição e com a duração do contato obtidos de valores de experimentos para determinados tipos de materiais e faixas de velocidade de impacto. As relações para os coeficientes de rigidez e de amortecimento podem ser derivadas analiticamente como uma função do coeficiente de restituição e da duração do contato (ver (HOEF et al., 2006; SHäFER; DIPPEL; WOLF, 1996)).

Na literatura existem diversos modelos não-lineares para a estimativa dos parâmetros de colisão durante o impacto (por exemplo, (TSUJI; TANAKA; ISHIDA, 1992; HUNT; GROSSLEY, 1975; KUWABARA; KONO, 1987)). A força conservativa nesses 
modelos é frequentemente calculada com base na teoria de Hertz (1882) e o coeficiente de rigidez não-linear é estimado pelas propriedades dos materiais, como o módulo de Young e o coeficiente de Poisson. A diferença básica entre os modelos está no cálculo da força de amortecimento não-conservativa. Tsuji, Tanaka e Ishida (1992) considera uma força de amortecimento proporcional a raiz quarta do "overlap" (sobreposição dos corpos) na simulação de partículas não coesivas em um duto horizontal. Hunt e Grossley (1975) consideram uma força contínua no início e no final do contato. Kuwabara e Kono (1987) propuseram uma força de amortecimento proporcional a raiz quadrada do "overlap" para a colisão de partículas esféricas viscoelásticas. Flores et al. (2011) realizaram uma análise de modelos contínuos para determinar a força de contato em problemas de dinâmica de multicorpos. Os autores derivaram uma relação explícita entre o coeficiente de restituição e o fator de amortecimento aplicando-a no problema clássico da queda-livre de uma bola.

No modelo linear, o valor do coeficiente de rigidez é frequentemente estimado de outros experimentos numéricos ou através de uma equivalência dos modelos lineares com os não-lineares (NAVARRO; BRAUN, 2013). O coeficiente de amortecimento é então determinado em função do coeficiente de restituição e da rigidez. Existem vários trabalhos que tratam da equivalência entre os modelos linear e não-linear para o cálculo da rigidez linear. Lan e Rosato (1995) utilizam a energia máxima equivalente para a avaliação da rigidez linear. Os autores assumem que a energia cinética incidente é armazenada na energia de deformação elástica Hertiziana e a rigidez da mola é obtida quando a energia não-linear é igualada à linear. Lan e Rosato (1997) usam uma sobreposição limite para determinar o coeficiente de rigidez. O valor da rigidez linear é associado com uma sobreposição máxima baseada em uma porcentagem do diâmetro da partícula. Dury e Ristow (1997) também determinam um valor para a rigidez linear da mola através de uma sobreposição limite calculada em função da porcentagem da soma dos raios das partículas. Buchholtz e Pöschel (1994) relacionam a rigidez linear com o módulo de Young caracterizando uma restituição elástica das esferas. Antypov e Elliott (2011) mapeiam o modelo não-linear ao linear ajustando as constantes lineares com as não-lineares.

Stevens e Hrenya (2005) discutem um critério para escolher valores típicos dos parâmetros de entrada (coeficiente de rigidez e coeficiente de amortecimento) baseados nos valores dos coeficientes de restituição e de amortecimento obtidos experimentalmente para um determinado valor da velocidade de impacto. Os autores sugerem utilizar uma velocidade de impacto média da faixa de dados experimentais. Os autores também comparam algumas relações da literatura (modelos lineares e não-lineares) com os dados experimentais, sendo que em algumas tanto o coeficiente de restituição quanto o tempo de contato diminuem com o aumento da velocidade de impacto.

As não-linearidades de ordem fracionária aparecem na modelagem das forças restauradoras em diversos problemas na literatura, como devido o contato dos corpos 
(modelos de Hertz), nas características elásticas dos materiais (alumínio, cobre, borracha), nas geometrias das molas (p.ex., molas helicoidais), nas geometrias dos problemas (como em mecanismos biela-manivela, treliças), entre outros problemas. Alves et al. (2015) apresentaram um estudo comparativo de diversos modelos da literatura para o cálculo da força de contato. Os autores examinaram as características fundamentais e o desempenho de cada modelo, em que foi dada ênfase para o processo dissipativo baseado no amortecimento interno. A propriedade elástica dos modelos analisados é baseada na teoria de contato de Hertz. Os resultados indicaram que a previsão do comportamento dinâmico durante o contato dos corpos depende em grande parte da seleção do modelo força de contato. Watanabe (1978) investiga a vibração forçada de sistemas contínuos com condições de contorno não-lineares. O autor estuda equipamentos tubulares de uma planta nuclear através da construção de um método analítico para a obtenção de soluções aproximadas. O autor propôs um modelo dinâmico do sistema contínuo equivalente a um sistema de um grau de liberdade em que ambos trataram a folga (impacto) como um sistema colidindo em uma mola linear de alta rigidez. Davies (1980) analisa vibrações aleatórias de uma viga colidindo em uma barreira elástica. O autor utiliza o processo de linearização para a obtenção da amplitude, velocidade e frequência durante o impacto mostrando que os resultados são coerentes com os obtidos por um sistema não-linear equivalente de um grau de liberdade. Shaw e Holmes (1983) consideram um sistema não-linear de um grau de liberdade em que a não-linearidade está presente em uma força elástica restauradora que é linear por partes devido ao processo de colisão do sistema mecânico. Os autores encontram no sistema analisado movimentos harmônicos, sub-harmônicos e caóticos derivados de bifurcações. Para a caracterização do impacto, os autores modelam o sistema mecânico colidindo com uma mola linear. Pilipchuk (2010) analisa em seu livro diversos problemas de osciladores com impacto. O autor apresenta a solução de problemas com não-linearidades fracionárias que é também detalhado no seu artigo (PILIPCHUK, 2007) que modela matematicamente e apresenta a solução para uma família de osciladores não-lineares em que a força elástica tem uma forma de potência generalizada e a força dissipativa é viscosa. Zhu e Ishitobi (2004) propõem um modelo para a suspensão de um veículo rodoviário considerando a mola e o amortecedor como não-lineares. A força elástica da mola é modelada como uma não-linearidade de ordem fracionária em que os expoentes utilizados são iguais a 1.25 e 1.5. Ario e Watson (2009) estudaram a estabilidade estrutural em treliças em que as não-linearidades fracionárias são decorrentes da geometria da estrutura e, também, devido ao contato entre os nós. Padthe et al. (2008) estudaram um mecanismo do tipo biela-manivela com histerese em que na derivação do modelo matemático aparece uma não-linearidade racional na força restauradora devido à geometria do problema. 



\section{Vibrações Mecânicas Não-lineares}

Neste capítulo são apresentadas soluções aproximadas e numéricas para osciladores fortemente não-lineares conservativos e não-conservativos.

\subsection{Vibração livre de osciladores conservativos com condições ini- ciais particulares}

Nesta seção é descrito um método para a obtenção de solução aproximada de osciladores conservativos com não-linearidades racionais e com condições iniciais particulares. Será utilizado a abordagem analítica aproximada Hamiltoniana (HE, 2010) para resolver sistemas fortemente não-lineares na forma de soma polinomial de ordem inteira ou não-inteira. A solução da equação diferencial não-linear é assumida na forma de uma função trigonométrica com frequência desconhecida. Usando a hipótese de que a derivada do Hamiltoniano em relação à amplitude de vibração é zero, uma equação para a frequência é obtida. A precisão da solução aproximada é tratada de duas formas: comparando com valores exatos do período de vibração obtidos numericamente e por um método de estimação do erro baseado na razão entre uma função residual média e a energia mecânica do sistema que é constante. O procedimento descrito na presente seção é aplicado a diversos tipos de exemplos como: oscilador com um termo fortemente não-linear; equação de Duffing; oscilador com não-linearidades do tipo polinomial, entre outros.

O oscilador analisado na presente seção tem não-linearidades do tipo polinomial com ordem que são números racionais. O modelo do oscilador é representado pela equação:

$$
\ddot{x}+f(x)=0,
$$

com as seguintes condições iniciais particulares:

$$
x(0)=A, \dot{x}(0)=0,
$$

onde $g(x)=-f(x)$ é uma força conservativa sendo $f(x)$ representado por uma soma polinomial de não-linearidades de ordem fracionária expressa por (ver (CVETICANIN; POGANY, 2012)):

$$
f(x)=\sum_{k=1}^{p} c_{k}^{2} x|x|^{\alpha_{k}-1},
$$

e $c_{k}^{2}$ são constantes positivas pequenas ou grandes e as ordens de não-linearidades de $\alpha_{k}$ são números racionais (inteiros ou não-inteiros), isto é, $\alpha_{k} \in \mathbb{Q}_{\geq 0}$. Para resolver a Eq. (3.1), a abordagem Hamiltoniana desenvolvida por He (2010) é extendida para obter uma relação 
entre a amplitude e a frequência no caso de osciladores mecânicos conservativos que são modelados por equações com soma de termos de potência de números inteiros e racionais positivos. A solução na forma trigonométrica é obtida com relação frequência-amplitude É necessário predizer a precisão da solução analítica aproximada obtida. Essa tarefa não é fácil, quando a solução exata na forma explícita não é conhecida. Para esse caso, usualmente, a solução aproximada é comparada com a obtida numericamente, considerada 'exata'. Assim, nesta seção, usando o método de Runge-Kutta, a solução numérica da Eq.(3.1) é calculada para determinadas condições iniciais (3.3). A solução numérica e a solução aproximada são comparadas e as diferenças são obtidas em determinados instantes. Outra maneira de comparar as soluções é tratar o erro da solução aproximada sem conhecer a solução exata do oscilador, isto é, de forma independente do tempo. Nesse caso, o erro médio entre a solução aproximada e a exata tem que ser determinado. Existem diversos procedimentos de estimativa de erro baseado no valor do resíduo quadrático médio (ver (FINLAYSON, 1972; ATANACKOVIC; ACHENBACH, 1985)). A principal desvantagem desses métodos é que eles são complexos e aplicados a determinadas classes de problemas. Recentemente, Farzaneh e Tootoonchi (2010) introduziu um método para a minimização do erro para a frequência do oscilador de Duffing. Na sequência, Akbarzade e Langari (2011) e Yazdi e Tehrani (2016) obtiveram sucesso para aplicar a técnica em diversos problemas nãolineares. Navarro e Cveticanin (2016) derivaram uma relação para a frequência aproximada utilizando a abordagem Hamiltoniana para osciladores com soma de não-linearidades de ordens não-inteiras. Os autores determinaram que um limite superior do erro na solução aproximada pode ser definido através do oscilador com um único termo e esse limite depende da ordem do oscilador.

Nesta seção também é apresentado um método para a estimativa de erro no qual não tem as limitações das mencionadas anteriormente. O método é baseado na média da função residual do oscilador conservativo. O erro é estimado como a integral do quadrado da diferença entre a energia mecânica aproximada e a total do oscilador, isto é, a energia correspondendo ao Hamiltoniano sobre o período de vibração. O erro obtido é uma função da amplitude inicial de vibração, dos coeficientes do modelo e das ordens de não-linearidades. O erro relativo é a razão entre a raiz quadrada da integral e a energia mecânica total inicial do oscilador. O método desenvolvido é aplicado em diversos exemplos e a precisão das soluções aproximadas é testada aplicando o procedimento proposto de estimativa de erro.

\subsubsection{Solução aproximada}

Considerando a Eq.(3.1), o trabalho $\delta W$ realizado no sistema por $-f(x)$ é dado por:

$$
\delta W=g(x) \delta x=-f(x) \delta x,
$$


quando o sistema movimenta da posição $x$ para uma posição vizinha $\delta(x)$. O trabalho $\delta W$ produz mudanças na energia cinética $K$ do sistema, onde $K=\frac{1}{2} \dot{x}^{2}$, isto é,

$$
\delta K=\delta W=-f(x) \delta x
$$

de forma que dividindo a Eq. (3.5) por $\delta(x)$ e tomando $\delta(x) \rightarrow 0$ obtém-se:

$$
\frac{d K(x)}{d x}=-f(x)
$$

Definindo uma função $F(x)$ dada pela relação

$$
\frac{d F(x)}{d x}=-g(x)=f(x)
$$

tem-se que $F(x)$ é a energia potencial total, e para a Eq. (3.3) essa energia é expressa por

$$
F(x)=\sum_{k=1}^{p} \frac{c_{\alpha_{k}}^{2}}{\alpha_{k}+1}|x|^{\alpha_{k}+1} .
$$

Somando as Eqs. (3.6) e (3.7), e integrando em relação a $x$ obtém-se

$$
K(x)+F(x)=H_{0}=\text { constante, }
$$

que representa a conservação da energia mecânica durante o movimento. A energia mecânica total do oscilador corresponde a seu Hamiltoniano:

$$
H=\frac{1}{2} \dot{x}^{2}+F(x)=\frac{1}{2} \dot{x}^{2}+\sum_{k=1}^{p} \frac{c_{k}^{2}}{\alpha_{k}+1}|x|^{\alpha_{k}+1} .
$$

Para osciladores conservativos, a energia mecânica total mantém-se inalterada durante a oscilação. Isso implicada que o Hamiltoniano é constante, isto é, $H=H_{0}=$ constante, e

$$
\frac{1}{2} \dot{x}^{2}+\sum_{k=1}^{p} \frac{c_{k}^{2}}{\alpha_{k}+1}|x|^{\alpha_{k}+1}-H_{0}=0,
$$

onde devido as condições iniciais (3.2)

$$
H_{0}=\sum_{k=1}^{p} \frac{c_{k}^{2}}{\alpha_{k}+1} A^{\alpha_{k}+1} .
$$

Muito frequentemente é impossível obter uma solução analítica fechada para a solução de (3.11). Portanto, assume-se uma solução tentativa na forma trigonométrica de primeira ordem:

$$
x(t)=A \cos (\omega t),
$$

onde $\omega$ é a frquência desconhecida de vibração e $A$ é a amplitude do oscilador. Substituindo a Eq. (3.13) na Eq. (3.10), obtém-se o Hamiltoniano aproximado do sistema:

$$
\tilde{H}=\frac{1}{2} A^{2} \omega^{2} \sin ^{2}(\omega t)+\sum_{k=1}^{p} \frac{c_{k}^{2}}{\alpha_{k}+1} A^{\alpha_{k}+1}\left[\sqrt{\cos ^{2}(\omega t)}\right]^{\alpha_{k}+1} .
$$


Normalmente, a frequência $\omega$ é determinada a partir da derivada da Eq. (3.14)

$$
\frac{\partial \tilde{H}}{\partial A}=0
$$

Infelizmente, o resultado obtido não tem uma boa precisão. Para tratar esse problema, He (2010) derivou um método chamado de abordagem Hamiltoniana. He (2010) introduziu uma nova função da forma:

$$
\bar{H}=\int_{0}^{T / 4} \tilde{H} d t
$$

onde $T$ é o período de vibração. Nesse caso considera-se que o Hamiltoniano é semelhante ('simétrico') a cada quarto de período. Caso isso não ocorra deve-se mudar o período de integração de forma adequada. Após a integração $\bar{H}=\tilde{H} T / 4$, de forma que:

$$
\tilde{H}=4 \frac{\partial \bar{H}}{\partial T}
$$

e de acordo com a Eq. (3.15) resulta em:

$$
\frac{\partial}{\partial A}\left(\frac{\partial \bar{H}}{\partial T}\right)=\frac{\partial}{\partial A}\left(\frac{\partial \bar{H}}{\partial \omega^{-1}}\right)=\frac{\partial}{\partial A}\left(\frac{\partial \bar{H}}{\partial \omega}\right)=0 .
$$

Resolvendo a equação algébrica (3.18), a frequência aproximada do oscilador nãolinear conservativo é obtida (ver Ref. (HE, 2010)). Dessa forma para

$$
\bar{H}(x, \dot{x})=\int_{0}^{T / 4}\left(\frac{1}{2} \dot{x}^{2}+\sum_{k=1}^{p} \frac{c_{k}^{2}}{\alpha_{k}+1}|x|^{\alpha_{k}+1}\right) d t,
$$

e usando a função tentativa (3.13), o Hamiltoniano aproximado, $\bar{H}$, é expresso como

$$
\bar{H}(A, \omega)=\int_{0}^{T / 4}\left\{\frac{1}{2} A^{2} \omega^{2} \sin ^{2}(\omega t)+\sum_{k=1}^{p} \frac{c_{k}^{2}}{\alpha_{k}+1}|A|^{\alpha_{k}+1}|\cos (\omega t)|^{\alpha_{k}+1}\right\} d t .
$$

Substituindo a variável $\psi=\omega t$ e o período de vibração $T=2 \pi / \omega$, a Eq. (3.20) é reescrita:

$$
\bar{H}(A, \omega)=\int_{0}^{\pi / 2}\left\{\frac{1}{2} A^{2} \omega \sin ^{2}(\psi)+\omega^{-1} \sum_{k=1}^{p} \frac{c_{k}^{2}}{\alpha_{k}+1}|A|^{\alpha_{k}+1}|\cos (\psi)|^{\alpha_{k}+1}\right\} d \psi .
$$

Considere a seguinte regra de integração (ver (ASKEY; ROY, 2010, página 142))

$$
\int_{0}^{\pi / 2} \sin ^{\mu-1}(\psi) \cos ^{\nu-1}(\psi) d \psi=\frac{1}{2} \mathrm{~B}\left(\frac{\mu}{2}, \frac{\nu}{2}\right),
$$

onde $\Re \mu>0, \Re \nu>0$ são partes reais positivas, e B é a função beta, também chamada de integral de Euler de primeiro tipo. Essa função especial é definida por (ver (ASKEY; ROY, 2010)) $\Re a>0, \Re b>0$ :

$$
\mathrm{B}(a, b)=\int_{0}^{1} t^{a-1}(1-t)^{b-1} d t=\frac{\Gamma(a) \Gamma(b)}{\Gamma(a+b)},
$$


onde $\Gamma$ é a função especial definida pela integral de Euler ( $z>0$ ) (ver (ASKEY; ROY, 2010, página 136)):

$$
\Gamma(z)=\int_{0}^{\infty} e^{-t} t^{z-1} d t
$$

que para um número inteiro positivo $n$ a Eq. (3.24) é dada por:

$$
\Gamma(n+1)=n !
$$

Assim, usando a Eq. (3.22) as integrais em (3.21) são escritas como:

$$
\int_{0}^{\pi / 2} \sin ^{2}(\psi) d \psi=\frac{\pi}{4}, \quad \int_{0}^{\pi / 2} \cos ^{\alpha_{k}+1}(\psi) d \psi=\frac{1}{2} \mathrm{~B}\left(\frac{1}{2}, \frac{\alpha_{k}+2}{2}\right) .
$$

Substituindo a Eq. (3.26) na Eq. (3.21), o Hamiltoniano é representado por:

$$
\bar{H}(A, \omega)=\frac{1}{2} A^{2} \omega \frac{\pi}{4}+\omega^{-1} \sum_{k=1}^{p} \frac{c_{k}^{2}}{\alpha_{k}+1}|A|^{\alpha_{k}+1} \frac{1}{2} \mathrm{~B}\left(\frac{1}{2}, \frac{\alpha_{k}+2}{2}\right) .
$$

de forma que usando a expressão (3.18) obtém-se:

$$
\frac{\partial}{\partial A}\left(\frac{\partial \bar{H}}{\partial \omega}\right) \equiv A \frac{\pi}{4}-\omega^{-2} \sum_{k=1}^{p} c_{k}^{2}|A|^{\alpha_{k}} \frac{1}{2} \mathrm{~B}\left(\frac{1}{2}, \frac{\alpha_{k}+2}{2}\right)=0 .
$$

Resolvendo a equação algébrica (3.28), a frequência aproximada de vibração, $\omega$, é obtida

$$
\omega=\sqrt{\frac{2}{\pi}}\left\{\sum_{k=1}^{p} c_{\alpha_{k}}^{2}|A|^{\alpha_{k}-1} \mathrm{~B}\left(\frac{1}{2}, \frac{\alpha_{k}+2}{2}\right)\right\}^{1 / 2} .
$$

Essa frequência depende dos coeficientes de rigidez do oscilador, $c_{k}^{2}$, das ordens de nãolinearidade, $\alpha_{k}$, e da amplitude inicial de vibração, $A$.

Usando a relação entre as funções especiais Beta B e Gamma $\Gamma$ (3.23) e a definição $\Gamma(1 / 2)=\sqrt{\pi}$, a expressão (3.29) para a frequência de vibração é reescrita como

$$
\omega=\frac{\sqrt{2}}{\pi^{1 / 4}}\left\{\sum_{k=1}^{p} c_{k}^{2}|A|^{\alpha_{k}-1} \frac{\Gamma\left(1+\frac{\alpha_{k}}{2}\right)}{\Gamma\left(\frac{\alpha_{k}+3}{2}\right)}\right\}^{1 / 2} .
$$

Assim, a solução aproximada da equação (3.1) com (3.3) é

$$
x(t)=A \cos \left(\frac{\sqrt{2}}{\pi^{1 / 4}}\left\{\sum_{k=1}^{p} c_{k}^{2}|A|^{\alpha_{k}-1} \frac{\Gamma\left(1+\frac{\alpha_{k}}{2}\right)}{\Gamma\left(\frac{\alpha_{k}+3}{2}\right)}\right\}^{1 / 2} t\right),
$$

e o período aproximado de vibração é

$$
T=\frac{2 \pi}{\omega}=\sqrt{2} \pi^{5 / 4}\left\{\sum_{k=1}^{p} c_{k}^{2}|A|^{\alpha_{k}-1} \frac{\Gamma\left(1+\frac{\alpha_{k}}{2}\right)}{\Gamma\left(\frac{\alpha_{k}+3}{2}\right)}\right\}^{-1 / 2} .
$$


Comparando a frequência aproximada de vibração (3.30) com o resultado obtido por Cveticanin et al. (2010, página 998), observa-se que ambas têm formas similares:

$$
\omega=\left\{\frac{\sum_{k=1}^{p} c_{k}^{2} a_{k}|A|^{\alpha_{k}-1}}{a_{0}}\right\}^{1 / 2}
$$

onde

$$
\begin{aligned}
& a_{0}=\frac{\pi}{4}, \\
& a_{k}=\frac{\pi}{2^{\alpha_{k}+1}\left(\alpha_{k}+1\right) \mathrm{B}\left(\frac{\alpha_{k}+3}{2}, \frac{\alpha_{k}+1}{2}\right)} .
\end{aligned}
$$

\subsubsection{Estimativa de erro da solução}

Nesta seção é desenvolvido um procedimento para estimar o erro da solução aproximada. No método proposto não é necessário conhecer a solução exata explicitamente e nem numericamente. O erro médio relativo é determinado sobre um período de vibração caracterizando a diferença na forma da resposta entre a solução aproximada e a exata. Assim substituindo a Eq. (3.13) na Eq. (3.10) tem-se:

$$
\tilde{H}=\frac{1}{2} A^{2} \omega^{2} \sin ^{2}(\omega t)+\sum_{k=1}^{p} \frac{c_{k}^{2}}{\alpha_{k}+1} A^{\alpha_{k}+1}|\cos (\omega t)|^{\alpha_{k}+1},
$$

onde a frequência de vibração $\omega$ é expressa pela Eq. (3.30) e $\tilde{H}$ é o Hamiltoniano calculado para a solução aproximada. É evidente que, para a solução aproximada (3.13), o Hamiltoniano (3.35) difere da energia mecânica total $H_{0}$. A diferença entre $\tilde{H}$ e $H_{0}$ fornece o seguinte resíduo:

$$
R(t)=\tilde{H}-H_{0}=\frac{1}{2} A^{2} \omega^{2} \sin ^{2}(\omega t)+\sum_{k=1}^{p} \frac{c_{k}^{2}}{\alpha_{k}+1} A^{\alpha_{k}+1}|\cos (\omega t)|^{\alpha_{k}+1}-\sum_{k=1}^{p} \frac{c_{k}^{2}}{\alpha_{k}+1} A^{\alpha_{k}+1} .
$$

Observa-se através da Eq. (3.36) que o resíduo varia com o tempo. A relação (3.36) fornece a diferença entre a energia calculada baseada na solução aproximada, para qualquer instante, e a energia total do oscilador.

Para determinar o valor médio, o erro é estimado como a integral do quadrado da diferença entre a energia mecânica calculada e a energia mecânica total, isto é, a integral do quadrado da função residual sobre o período de vibração. Para fazer essa estimativa o seguinte funcional é introduzido:

$$
\Delta_{2}=\frac{1}{T} \int_{0}^{T} R(t)^{2} d t=\frac{1}{T} \int_{0}^{T}\left(\tilde{H}-H_{0}\right)^{2} d t
$$

Substituindo a Eq. (3.36) na Eq. (3.37) obtém-se

$$
\Delta_{2}=\frac{4}{T} \int_{0}^{T / 4}\left[\frac{1}{2} A^{2} \omega^{2} \sin ^{2}(\omega t)+\sum_{k=1}^{p} \frac{c_{k}^{2}}{\alpha_{k}+1} A^{\alpha_{k}+1}|\cos (\omega t)|^{\alpha_{k}+1}-\sum_{k=1}^{p} \frac{c_{k}^{2}}{\alpha_{k}+1} A^{\alpha_{k}+1}\right]^{2} d t
$$


e para $\psi=\omega t$ e $T=2 \pi / \omega$ :

$$
\Delta_{2}=\frac{2}{\pi} \int_{0}^{\pi / 2}\left[\frac{1}{2} A^{2} \omega^{2} \sin ^{2}(\psi)+\sum_{k=1}^{p} \frac{c_{k}^{2}}{\alpha_{k}+1} A^{\alpha_{k}+1}|\cos (\psi)|^{\alpha_{k}+1}-\sum_{k=1}^{p} \frac{c_{k}^{2}}{\alpha_{k}+1} A^{\alpha_{k}+1}\right]^{2} d \psi .
$$

A solução obtida é uma função da amplitude inicial, ordens de não-linearidades e coeficientes de não-linearidade: $\Delta_{2}=\Delta_{2}\left(A, c_{k}, \alpha_{k}\right)$. O erro relativo é calculado como a razão entre a raiz quadrada da função residual média e a função da energia mecância inicial que é constante, $H_{0}$

$$
\Delta=\frac{\sqrt{\Delta_{2}}}{H_{0}} .
$$

É possível representar o erro relativo na forma percentual por: $\Delta_{\%}=\left(\frac{\sqrt{\Delta_{2}}}{H_{0}}\right) 100 \%$.

\subsubsection{Comparação entre as soluções aproximada e exata}

\subsubsection{Equação com um termo conservativo}

Considerando um oscilador mecânico com um único termo não-conservativo, também chamado de oscilador puro, a Eq. (3.1) para $p=1$ é representada por:

$$
\ddot{x}+c_{1}^{2} x|x|^{\alpha_{1}-1}=0 .
$$

De acordo com a Eq. (3.31), a solução aproximada é

$$
x(t)=A \cos (\omega t),
$$

onde

$$
\omega^{2}=c_{1}^{2}|A|^{\alpha_{1}-1} K_{\alpha 1}
$$

e

$$
K_{\alpha 1}=\frac{2}{\pi} B\left(\frac{1}{2}, \frac{\alpha_{1}+2}{2}\right) .
$$

Dessa forma, a solução aproximada pode ser expressa por:

$$
x(t)=A \cos \left(\frac{\sqrt{2}}{\pi^{1 / 4}}\left\{c_{1}^{2}|A|^{\alpha_{1}-1} \frac{\Gamma\left(1+\frac{\alpha_{1}}{2}\right)}{\Gamma\left(\frac{\alpha_{1}+3}{2}\right)}\right\}^{1 / 2} t\right)
$$

e o período aproximado de vibração, $T$, é

$$
T=\frac{K_{1}}{\left|c_{1}\right||A|^{\left(\alpha_{1}-1\right) / 2}}
$$

onde

$$
K_{1}=\sqrt{2} \pi^{5 / 4} \sqrt{\frac{\Gamma\left(\frac{\alpha_{1}+3}{2}\right)}{\Gamma\left(1+\frac{\alpha_{1}}{2}\right)}} .
$$


O período exato de vibração da Eq. (3.41), de acordo com Cveticanin (2009b, página 1067) e Cveticanin e Pogany (2012, página 4), é expresso por:

$$
T_{e x}=\frac{K_{2}}{\left|c_{1}\right||A|^{\left(\alpha_{1}-1\right) / 2}}
$$

onde

$$
K_{2}=\frac{2 \sqrt{2 \pi}}{\sqrt{\alpha_{1}+1}} \frac{\Gamma\left(\frac{1}{\alpha_{1}+1}\right)}{\Gamma\left(\frac{3+\alpha_{1}}{2\left(\alpha_{1}+1\right)}\right)} .
$$

A solução exata para a Eq. (3.41) é derivada por Cveticanin e Pogany (2012, página 8) para valores gerais de $\alpha_{1}>0$ por meio da solução por funções-Ateb, como:

$$
x_{\text {ex }}(t)=A \cdot \operatorname{ca}\left(\alpha_{1}, 1, \frac{\sqrt{\alpha_{1}+1}\left|c_{1}\right|}{\sqrt{2}} A^{\left(\alpha_{1}-1\right) / 2} t\right),
$$

onde ca $(\alpha, 1, z)$ é a função-Ateb coseno, que é par e $\Pi_{\alpha}$-periódica com $\Pi_{\alpha}=\mathrm{B}\left(\frac{1}{\alpha+1}, \frac{1}{2}\right)$, ver (CVETICANIN; POGANY, 2012, página 7) e (ROSENBERG, 1963). Rosenberg (1963) propôs as funções Ateb(h) que são inversões das funções incompleta Beta. Elas são utilizadas para expressar soluções de vibrações de sistemas não-lineares de múltiplos graus de liberdade como as funções trigonométricas são as soluções dos correspondentes sistemas lineares. Segundo Rosenberg (1963), as funções Ateb reduzem para as funções trigonométricas como as funções Atebh para as funções hiperbólicas com o parâmetro 1. Quando o parâmetro é igual a 2 elas reduzem para as funções elípticas. As funções Ateb(h) têm diversas propriedades como identidades, derivadas, integrais, equações diferenciais, entre outras.

\section{Caso $\alpha_{1}=1$ :}

Quando o oscilador for linear $\left(\alpha_{1}=1\right)$, as soluções exatas e aproximadas são equivalentes. Considerando a Eq. (3.50) onde ca $(1,1, z)=\cos z$ e Eq. 3.42 onde $\Gamma\left(\frac{3}{2}\right)=\frac{\sqrt{\pi}}{2}$ e $\Gamma(2)=1$, as soluções são escritas como $x(t)=x_{e x}(t)=A \cdot \cos \left(\left|c_{1}\right| t\right)$, com o período de vibração expresso pelas equações (3.46) e (3.48) $\operatorname{como} T=T_{e x}=\frac{2 \pi}{\left|c_{1}\right|} \operatorname{com} \Gamma\left(\frac{1}{2}\right)=\sqrt{\pi}$ e $\Gamma(1)=1$, que é o período obtido da teoria de vibrações lineares.

Caso $\alpha_{1}=3$ :

A solução exata para a equação cúbica $\left(\alpha_{1}=3\right)$ é (Eq. 3.50)

$$
x_{e x}(t)=A \cdot \operatorname{ca}\left(3,1, \frac{\sqrt{3+1}\left|c_{1}\right|}{\sqrt{2}} A^{(3-1) / 2} t\right)=A \cdot \operatorname{ca}\left(3,1, \sqrt{2}\left|c_{1}\right| A t\right),
$$

e a solução exata na forma das funções elípticas de Jacobi (ver (BYRD; FRIEDMAN, 1971)) é expressa por ((CVETICANIN; POGANY, 2012), (CVETICANIN, 2009b), (COPPOLA; 
RAND, 1990)):

$$
x_{e x}(t)=A \cdot \operatorname{cn}\left(\Omega t, k^{2}\right),
$$

onde $\Omega=\left|c_{1}\right| A$ e $k^{2}=\frac{1}{2}$. Usando a Eq. (3.48) ou o fato de que a função elíptica, $\operatorname{cn}\left(u, k^{2}\right)$, é periódica com período $4 K\left(k^{2}\right)$, onde $K\left(k^{2}\right)$ é a integral elíptica completa de primeiro tipo (BYRD; FRIEDMAN, 1971), o período exato de vibração é (CVETICANIN, 2009b):

$$
T_{e x}=\frac{\sqrt{2 \pi}}{\left|c_{1}\right||A|}\left\{\frac{\Gamma\left(\frac{1}{4}\right)}{\Gamma\left(\frac{3}{4}\right)}\right\}=\frac{4 K\left(\frac{1}{2}\right)}{\left|c_{1}\right||A|}=\frac{7.4163}{\left|c_{1}\right||A|} .
$$

Utilizando a Eq. (3.42), a solução aproximada para a equação do oscilador cúbico puro é:

$$
x(t)=A \cos \left(0.866\left|c_{1} \| A\right| t\right),
$$

onde o período aproximado é expresso usando a Eq. (3.46) e para $\Gamma(5 / 2)=3 \sqrt{\pi} / 4, \Gamma(3)=2$ tem-se:

$$
T=\frac{\sqrt{2} \pi^{5 / 4}}{\left|c_{1}\right||A|}\left\{\frac{\Gamma(3)}{\Gamma\left(\frac{5}{2}\right)}\right\}^{1 / 2}=\frac{7.2552}{\left|c_{1}\right||A|} .
$$

sendo que o erro em porcentagem calculado pela Eq. (3.56) é $2.1723 \%$.

\section{Caso $\alpha_{1} \geqslant 0$ :}

Através das relações (3.47) e (3.49) calculam-se os parâmetros $K_{1}$ e $K_{2}$ para diversos valores de $\alpha_{1}$. A Figura (1) ilustra a variação dos parâmetro $K_{1}$ e $K_{2}$ versus as potências $\alpha_{1}$. Observa-se que para $\alpha_{1} \leq 3$ ocorre uma boa concordância entre as soluções aproximada e exata. Para altos valores de $\alpha_{1}$ a diferença entre as soluções aumenta.

A seguir para comparar o período exato $T_{e x}$ (3.48) com o aproximado $T$ (3.46), determina-se o erro em porcentagem da forma:

$$
\left|\frac{T_{e x}-T}{T_{e x}}\right| \cdot 100 \%=\left|1-\frac{K_{1}}{K_{2}}\right| \cdot 100 \% .
$$

Na Tabela 1 são apresentados os valores dos parâmetros $K_{1}$ e $K_{2}$, e também o erro relativo (3.56) para diversos valores de $\alpha_{1}$.

Observa-se o aumento significante do período de vibração aproximado com o aumento da ordem de não-linearidade $\alpha_{1}$. Dessa forma, o presente método é adequado para valores da ordem de não-linearidade até aproximadamente $\alpha_{1}=5$. 


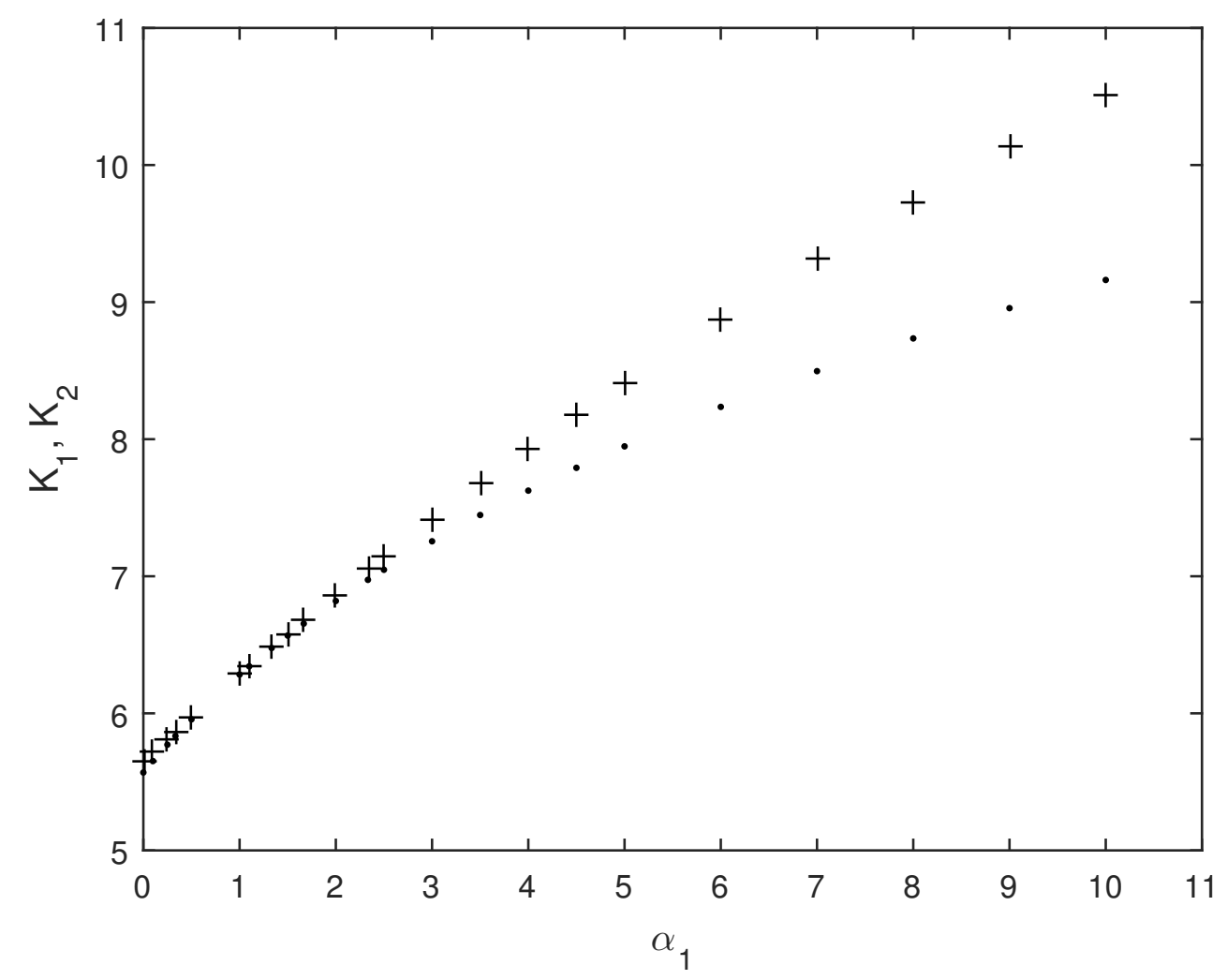

Figura 1 - Parâmetros $K_{1}(\cdot)$ e $K_{2}(+)$ versus a ordem de não-linearidade $\alpha_{1}$. 
Tabela 1 - Valores dos parâmetros $K_{1}, K_{2}$ e erros relativos (Eq. 3.56) para valores de $\alpha_{1}$.

\begin{tabular}{llll}
\hline$\alpha_{1}$ & $K_{1}$ & $K_{2}$ & Erro \% \\
\hline 0 & 5.56833 & 5.65685 & 1.5649 \\
$1 / 10$ & 5.65200 & 5.72105 & 1.2069 \\
$1 / 4$ & 5.77138 & 5.81679 & 0.7807 \\
$1 / 3$ & 5.83481 & 5.86966 & 0.5938 \\
$1 / 2$ & 5.95613 & 5.97467 & 0.3103 \\
1 & 6.28319 & 6.28319 & 0.0000 \\
1.1 & 6.34304 & 6.34366 & 0.0098 \\
$4 / 3$ & 6.47666 & 6.48313 & 0.0997 \\
$3 / 2$ & 6.56743 & 6.58136 & 0.2117 \\
$5 / 3$ & 6.65468 & 6.67845 & 0.3560 \\
2 & 6.81978 & 6.86926 & 0.7203 \\
$7 / 3$ & 6.97393 & 7.05571 & 1.1591 \\
$5 / 2$ & 7.04739 & 7.14736 & 1.3987 \\
3 & 7.25520 & 7.41630 & 2.1723 \\
$7 / 2$ & 7.44676 & 7.67673 & 2.9956 \\
4 & 7.62475 & 7.92929 & 3.8407 \\
$9 / 2$ & 7.79118 & 8.17456 & 4.6899 \\
5 & 7.94767 & 8.41309 & 5.5321 \\
6 & 8.23567 & 8.87180 & 7.1703 \\
7 & 8.49642 & 9.30874 & 8.7265 \\
8 & 8.73525 & 9.72663 & 10.1925 \\
9 & 8.95601 & 10.12768 & 11.5690 \\
10 & 9.16160 & 10.51373 & 12.8606 \\
\hline & & &
\end{tabular}




\section{Estimativa do erro:}

Para estimar o erro médio da solução aproximada durante um período, o Hamiltoniano da equação (3.41) assume a seguinte forma:

$$
H=\frac{1}{2} \dot{x}^{2}+\frac{1}{\alpha_{1}+1} c_{1}^{2}|x|^{\alpha_{1}+1} .
$$

Considerando as condições iniciais particulares $x(0)=A$ e $\dot{x}(0)=0$, a energia mecânica constante do sistema é:

$$
H_{0}=\frac{1}{\alpha_{1}+1} c_{1}^{2} A^{\alpha_{1}+1} .
$$

Substituindo a solução aproximada (3.42) em (3.57) e usando a relação (3.37), a função de erro de acordo com (3.39) é representada por:

$$
\Delta_{2}=\frac{2}{\pi} \int_{0}^{\pi / 2}\left[\frac{1}{2} A^{2} \omega^{2} \sin ^{2}(\psi)+\frac{c_{1}^{2}}{\alpha_{1}+1} A^{\alpha_{1}+1}|\cos (\psi)|^{\alpha_{1}+1}-\frac{c_{1}^{2}}{\alpha_{1}+1} A^{\alpha_{1}+1}\right]^{2} d \psi .
$$

Após alguns cálculos a função para o erro (3.59) é reescrita como:

$$
\Delta_{2}=c_{1}^{4} A^{2\left(\alpha_{1}+1\right)} K_{3}
$$

onde o parâmetro $K_{3}$ é expresso por

$$
K_{3}=\frac{1-2 C_{1}+C_{2}}{\left(\alpha_{1}+1\right)^{2}}+\frac{K_{\alpha 1}^{2} S_{4}}{4}+\frac{K_{\alpha 1}\left(C_{1}-C_{3}-S_{2}\right)}{\alpha_{1}+1},
$$

com coeficientes

$$
\begin{aligned}
& C_{1}=\frac{2}{\pi} \int_{0}^{\pi / 2} \cos ^{\alpha_{1}+1}(\psi) d \psi=\frac{1}{\pi} B\left(\frac{1}{2}, \frac{\alpha_{1}+2}{2}\right), \\
& C_{2}=\frac{2}{\pi} \int_{0}^{\pi / 2} \cos ^{2\left(\alpha_{1}+1\right)}(\psi) d \psi=\frac{1}{\pi} B\left(\frac{1}{2}, \frac{2 \alpha_{1}+3}{2}\right), \\
& C_{3}=\frac{2}{\pi} \int_{0}^{\pi / 2} \cos ^{\alpha_{1}+3}(\psi) d \psi=\frac{1}{\pi} B\left(\frac{1}{2}, \frac{\alpha_{1}+4}{2}\right), \\
& S_{4}=\frac{2}{\pi} \int_{0}^{\pi / 2} \sin ^{4}(\psi) d \psi=\frac{1}{\pi} B\left(\frac{1}{2}, \frac{5}{2}\right)=\frac{3}{8}, \\
& S_{2}=\frac{2}{\pi} \int_{0}^{\pi / 2} \sin ^{2}(\psi) d \psi=\frac{1}{\pi} B\left(\frac{1}{2}, \frac{3}{2}\right)=\frac{1}{2} .
\end{aligned}
$$

De acordo com (3.40) e (3.60), o erro relativo (3.40) é dado por

$$
\Delta=\frac{\sqrt{\Delta_{2}}}{H_{0}}=\left(\alpha_{1}+1\right) \sqrt{K_{3}}
$$


$\mathrm{Na}$ Tabela 2, o valor do erro relativo $\Delta$ é mostrado para diversos valores de $\alpha_{1}$. De acordo com a Tabela 2 é evidente que o erro é zero para o oscilador linear $\left(\alpha_{1}=1\right)$ quando o Hamiltoniano é calculado de acordo com a solução exata da equação (3.41). Observa-se, também, que o erro aumenta para valores maiores e menores do que a ordem de não-linearidade $\alpha_{1}=1$.

Tabela 2 - Valores dos erros relativos para diversas ordens de não-linearidade $\alpha_{1}$

\begin{tabular}{|l|l|l|l|l|l|l|l|l|l|l|l|l|}
\hline$\alpha_{1}$ & 0 & $1 / 2$ & 1 & $3 / 2$ & 2 & $5 / 2$ & 3 & $7 / 2$ & 4 & 6 & 8 & 10 \\
\hline$\Delta$ & 0.11096 & 0.05957 & 0 & 0.06079 & 0.12047 & 0.17823 & 0.23385 & 0.28736 & 0.33886 & 0.52752 & 0.69433 & 0.84502 \\
\hline
\end{tabular}

\subsubsection{Equação com diversos termos conservativos}

Na presenta seção são comparados a solução aproximada, Eq. (3.31), e o período de vibração, Eq. (3.32), com os valores obtido numericamente. O período de vibração numérico 'exato' numérico, $T_{n}$, é calculado usando a solução numérica da equação Eq. (3.1) com as condições iniciais $x(0)=A, \dot{x}(0)=0$ por meio do método de Runge-Kutta. O histórico no tempo da variável $x$ corresponde a um quarto do período a ser usado nos cálculos. Na sequência são ilustrados alguns exemplos de equações envolvendo mais do que um termo conservativo.

\section{Oscilador de Duffing:}

Seja um oscilador mecânico do tipo Duffing com não-linearidades cúbicas representado por:

$$
\ddot{x}+c_{1}^{2} x+c_{2}^{2} x^{3}=0,
$$

a frequência aproximada obtida da equação (3.30) para $p=2, \alpha_{1}=1, \alpha_{2}=3$ e $\Gamma(3 / 2)=$ $\sqrt{\pi} / 2, \Gamma(5 / 2)=3 \sqrt{\pi} / 4, \Gamma(2)=1, \Gamma(3)=2$ é escrita como:

$$
\omega=\frac{\sqrt{2}}{\pi^{1 / 4}}\left\{c_{1}^{2} \frac{\Gamma\left(\frac{3}{2}\right)}{\Gamma(2)}+c_{2}^{2}|A|^{2} \frac{\Gamma\left(\frac{5}{2}\right)}{\Gamma(3)}\right\}^{1 / 2}=\sqrt{c_{1}^{2}+\frac{3}{4} c_{2}^{2}|A|^{2}},
$$

que é o mesmo valor aproximado mostrado por ((HE, 2002, página 108), (HE, 2007, página 1434), (HE, 2010, página 2313)). A solução analítica exata para a equação de Duffing com não-linearidades cúbicas é escrita na forma de funções elípticas de Jacobi, de acordo com Cveticanin (2009a, páginas 1345,1346)), como:

$$
x_{e x}(t)=A \cdot \operatorname{cn}\left(\Omega t, k^{2}\right),
$$

onde $\Omega=\sqrt{c_{1}^{2}+c_{2}^{2} A^{2}}$ e $k^{2}=\frac{c_{2}^{2} A^{2}}{2 \Omega^{2}}$. O período de vibração exato é $T_{e x}=\frac{4 K(k)}{\Omega}$. He $(2002$, página 109) mostra que para $c_{1}^{2}=1$ e qualquer valor de $c_{2}^{2}>0$ o erro relativo máximo, 


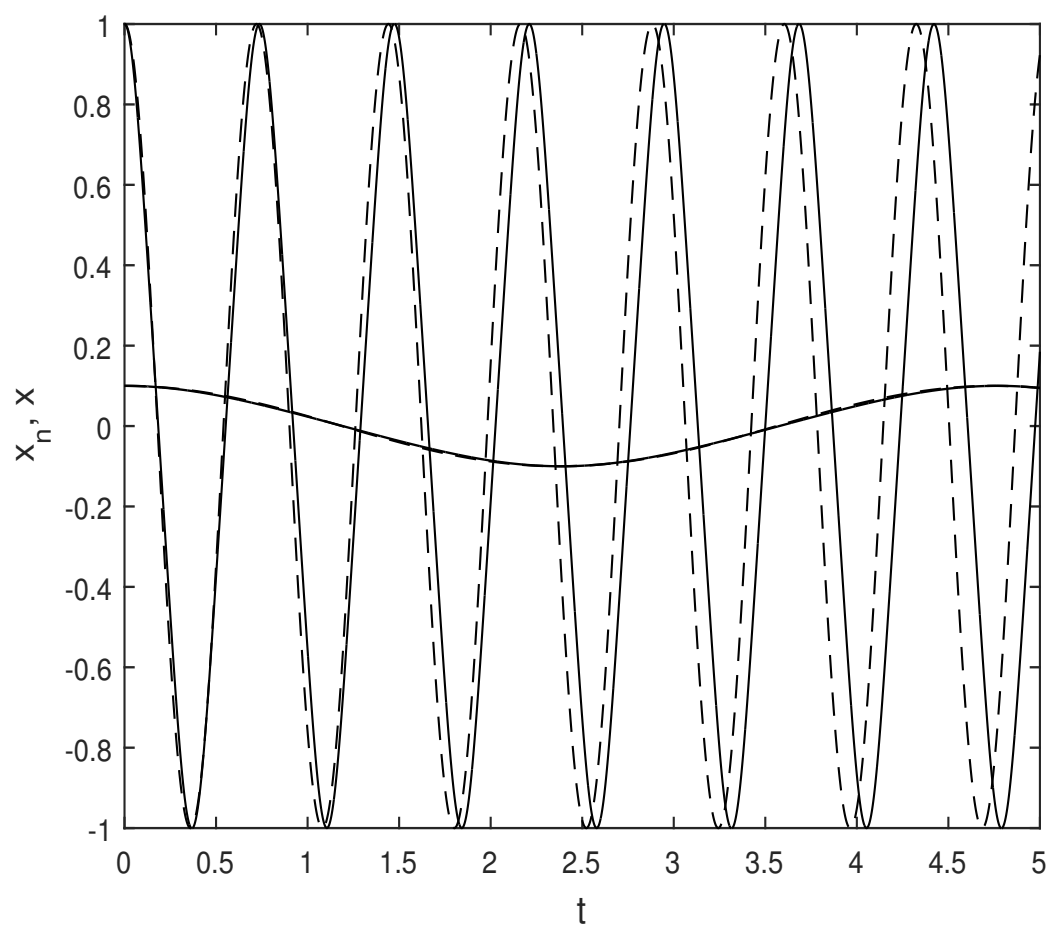

Figura 2 - Solução numérica 'exata', $x_{n}$, (linha contínua) e solução aproximada, $x$, (linha tracejada) para $A=1$ e para $A=0.1\left(c_{1}^{2}=1, c_{2}^{2}=100\right)$.

$\left|\left(T_{e x}-T\right) / T_{e x}\right| \cdot 100 \%<7.6 \%$. O período de vibração numérico é calculado usando RK45 com $0 \leqslant t \leqslant 1.5$ e incremento temporal de $\Delta t=10^{-6}$. Os períodos de vibração são calculados para duas condições iniciais distintas: $A=1$ e $A=0.1$ e para os parâmetros $c_{1}^{2}=1, c_{2}^{2}=100$. Para a amplitude inicial de vibração, $A=1$, os seguintes valores são obtidos: $T=$ $0.72073, T_{e x}=0.73629, T_{n}=0.73636$ e o erro relativo de $\left|\left(T_{e x}-T\right) / T_{e x}\right| \cdot 100 \%=2.113 \%$, $\left|\left(T_{n}-T\right) / T_{n}\right| \cdot 100 \%=2.123 \%$, e $\left|\left(T_{e x}-T_{n}\right) / T_{e x}\right| \cdot 100 \%=0.010 \%$. Para a condição inicial $A=0.1$, os períodos de vibração são: $T=4.7496, T_{e x}=4.7680, T_{n}=4.7680 \mathrm{e}$ os erros relativos de $\left|\left(T_{e x}-T\right) / T_{e x}\right| \cdot 100 \%=0.385 \%,\left|\left(T_{n}-T\right) / T_{n}\right| \cdot 100 \%=0.385 \%$, e $\left|\left(T_{e x}-T_{n}\right) / T_{e x}\right| \cdot 100 \%=0.000 \%$.

A Figura 2 ilustra duas soluções para o intervalo temporal de $(0 \leqslant t \leqslant 5.0)$ : 'exata' (linha contínua) e aproximada (linha tracejada). A solução aproximada é calculada com o método usando um incremento temporal de $\Delta t=10^{-6}$. A Figura 2 mostra as soluções para diferentes condições iniciais $(A=1$ e $A=0.1)$. Para a amplitude inicial $A=1$, a diferença entre as soluções aproximada e numérica é significante para tempos longos. Enquanto para a amplitude inicial de $A=0.1$, a diferença entre as soluções é desprezível. 


\section{Equação de Duffing com não-linearidade quadrática simétrica:}

Seja a seguinte equação de Duffing cúbica com não-linearidade de ordem quadrática simétrica:

$$
\ddot{x}+c_{1}^{2} x+c_{2}^{2} x^{3}+c_{3}^{2} x|x|=0,
$$

derivada a partir da equação Eq. (3.3) para $p=3, \alpha_{1}=1, \alpha_{2}=3, \alpha_{3}=2$. A frequência aproximada (Eq. 3.30) com $\Gamma(3 / 2)=\sqrt{\pi} / 2, \Gamma(5 / 2)=3 \sqrt{\pi} / 4, \Gamma(2)=1, \Gamma(3)=2$ é expressa por:

$$
\begin{aligned}
\omega & =\frac{\sqrt{2}}{\pi^{1 / 4}}\left\{c_{1}^{2} \frac{\Gamma\left(\frac{3}{2}\right)}{\Gamma(2)}+c_{2}^{2}|A|^{2} \frac{\Gamma\left(\frac{5}{2}\right)}{\Gamma(3)}+c_{3}^{2}|A| \frac{\Gamma(2)}{\Gamma\left(\frac{5}{2}\right)}\right\}^{1 / 2} \\
& =\sqrt{c_{1}^{2}+\frac{3}{4} c_{2}^{2}|A|^{2}+\frac{8}{3 \pi} c_{3}^{2}|A|},
\end{aligned}
$$

onde o período de vibração aproximado é $T=2 \pi / \omega$. A seguir compara-se a abordagem Hamiltoniana, (HE, 2010), com quatro métodos aproximados investigados por Cveticanin (2009a) baseados nas funções elípticas de Jacobi. Esses métodos são: método do balanço harmônico elíptico (EHBM), método de Galerkin elíptico (EGM), método de expansão direta (SFEM), e método de Lindstedt-Poincare elíptico (ELPM). A Tabela 3 mostra os períodos de vibração obtidos por esses métodos (HM - método Hamiltoniano; EHBM; EGM; SFEM; e ELPM) para valores particulares dos parâmetros do sistema e de amplitudes iniciais $\left(c_{1}^{2}=c_{2}^{2}=1, c_{3}^{2}=0.1, A=0.1\right) ;\left(c_{1}^{2}=c_{2}^{2}=c_{3}^{2}=1, A=0.1\right)$; e $\left(c_{1}^{2}=c_{2}^{2}=c_{3}^{2}=1\right.$, e $A=1)$. Os erros relativos são calculados por $\left|\left(T_{n}-T\right) / T_{n}\right| \cdot 100 \%$ onde $T_{n}$ é dado por (CVETICANIN, 2009a, página 1358).

Tabela 3 - Período de vibração e erros relativos para diversos métodos aproximados (adaptado de Cveticanin (2009a, página 1358)).

\begin{tabular}{llccccc}
\hline Método & Período & Erro \% & Período & Erro \% & Período & Erro \% \\
Parâmetros & $c_{1}^{2}=c_{2}^{2}=1, c_{3}^{2}=0.1$ & \multicolumn{2}{c}{$\begin{array}{c}c_{1}^{2}=c_{2}^{2}=c_{3}^{2}=1 \\
c_{1}^{2}=c_{2}^{2}=c_{3}^{2}=1\end{array}$} \\
$\begin{array}{llccccc}\text { A } \\
\text { HM }\end{array}$ & 6.2336 & 0.039592 & 6.0116 & 0.006354 & 3.8975 & 0.495290 \\
EHBM & 6.2337 & 0.041196 & 6.0122 & 0.003626 & 3.9139 & 0.076591 \\
EGM & 6.2334 & 0.036382 & 6.0119 & 0.001364 & 3.9173 & 0.010212 \\
SFEM & 6.2357 & 0.073293 & 6.0266 & 0.243150 & 4.4686 & 14.08500 \\
ELPM & 6.2357 & 0.073293 & 6.0201 & 0.135030 & 4.3926 & 12.14500 \\
Numérico & 6.231133 & & 6.011982 & & 3.9169 & \\
\hline
\end{tabular}

Comparando os erros relativos obtidos (Tabela 3), os métodos Hamiltoniano, balanço harmônico elíptico e Galerkin elíptico forneceram resultados precisos independentemente das condições inicias e dos valores dos parâmetros do sistema. Os métodos 


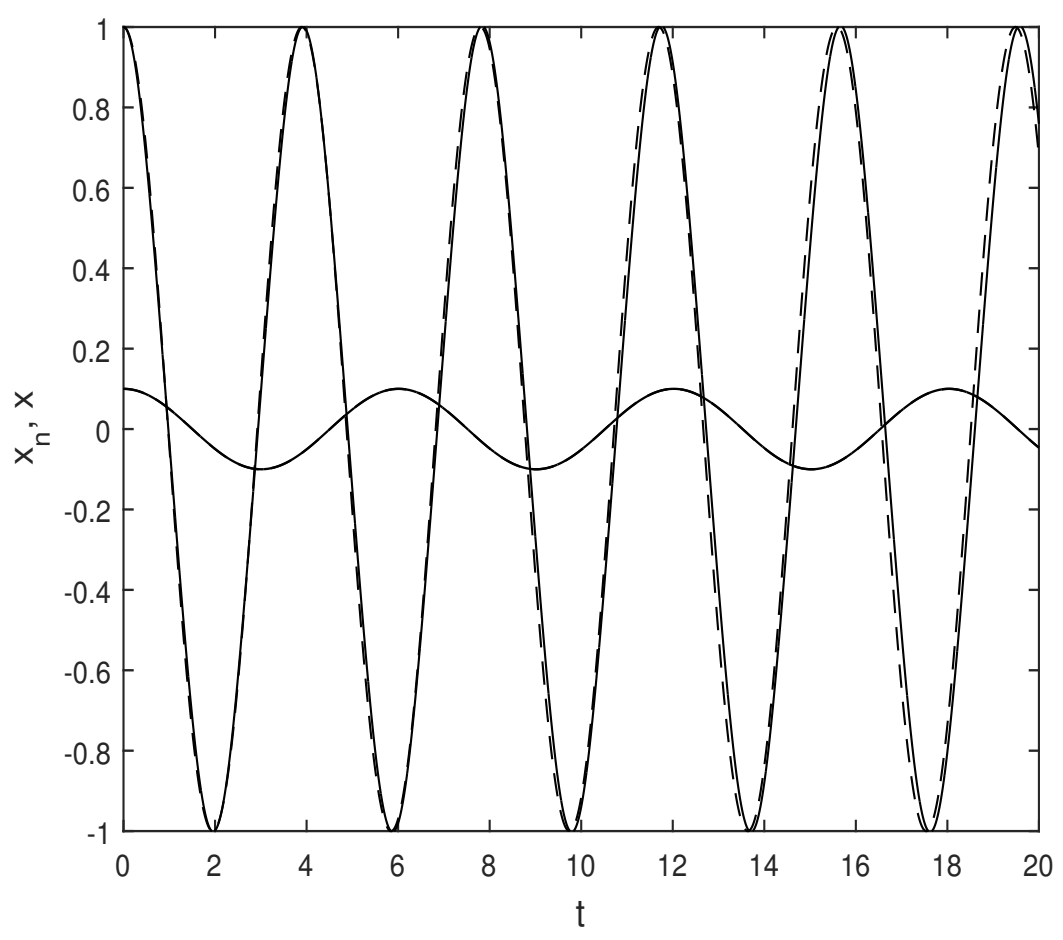

Figura 3 - Solução numérica 'exata', $x_{n}$, (linha contínua) e solução aproximada, $x$, (linha tracejada) para $A=1$ e para $A=0.1\left(c_{1}^{2}=c_{2}^{2}=c_{3}^{2}=1\right)$.

de expansão direta e o Lindsted-Poincare elíptico forneceram os piores resultados para o caso $c_{1}^{2}=c_{2}^{2}=c_{3}^{2}=1$ e $A=1$. A Figura 3 ilustra as soluções numérica (linha contínua) e aproximada (linha tracejada) para duas condições iniciais diferentes $(A=1$ e $A=0.1)$ durante o intervalo de tempo $\left(0 \leqslant t \leqslant 20 \mathrm{com} \Delta t=10^{-6}\right)$. Em ambos casos, as soluções são próximas com uma boa concordância entre elas.

\section{Não-linearidades de ordem ímpar:}

Nesta seção é considerado um oscilador mecânico com não-linearidades de ordem ímpares do tipo cúbica, quinta e sétima representado pela seguinte equação:

$$
\ddot{x}+c_{1}^{2} x+c_{2}^{2} x^{3}+c_{3}^{2} x^{5}+c_{4}^{2} x^{7}=0,
$$

a frequência de vibração aproximada obtida pela equação Eq. (3.30) para $p=4\left(\alpha_{1}=\right.$ $1, \alpha_{2}=3, \alpha_{3}=5$, e $\left.\alpha_{4}=7\right) \operatorname{com} \Gamma(7 / 2)=15 \sqrt{\pi} / 8, \Gamma(9 / 2)=105 \sqrt{\pi} / 16, \Gamma(4)=6, \Gamma(5)=$ 24 é escrita como: 


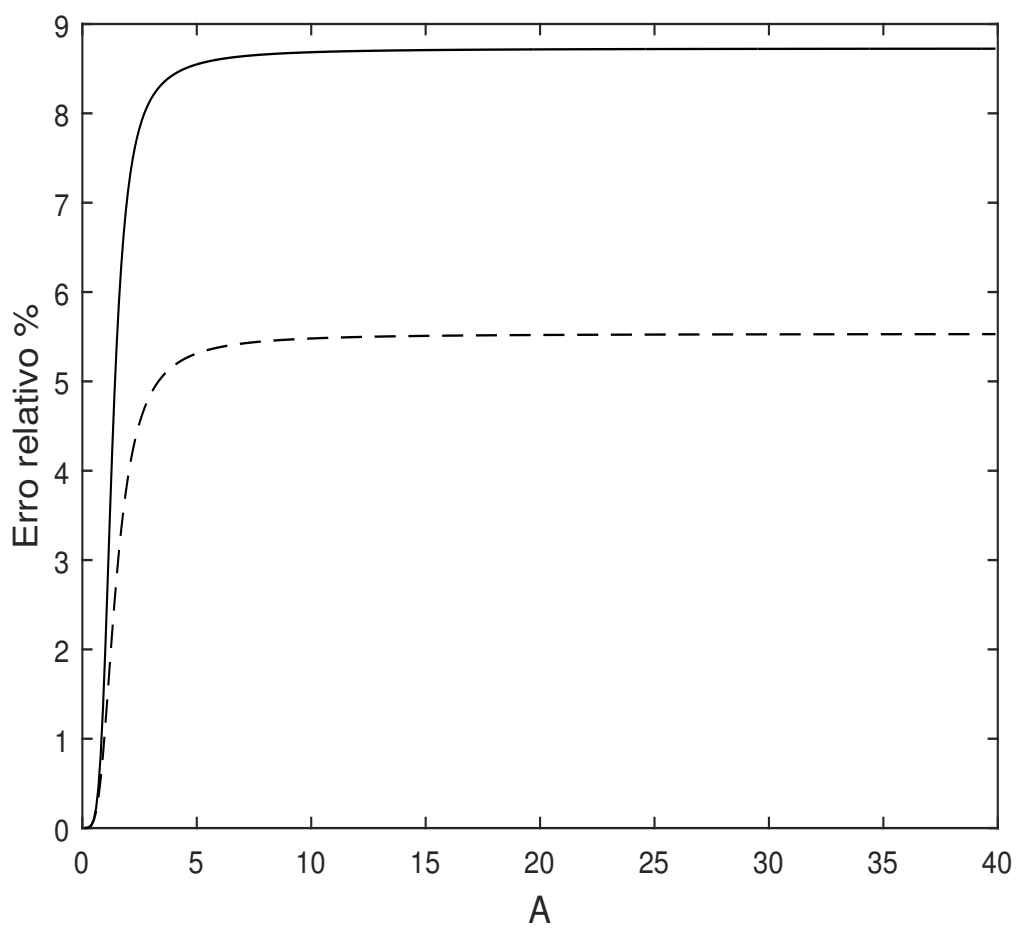

Figura 4 - Erro relativo (\%) versus amplitude, $A$, para $c_{1}^{2}=c_{2}^{2}=c_{3}^{2}=c_{4}^{2}=1$ (linha contínua) e $c_{1}^{2}=c_{2}^{2}=c_{3}^{2}=1, c_{4}^{2}=0$ (linha tracejada).

$$
\begin{aligned}
\omega & =\frac{\sqrt{2}}{\pi^{1 / 4}}\left\{c_{1}^{2} \frac{\Gamma\left(\frac{3}{2}\right)}{\Gamma(2)}+c_{2}^{2}|A|^{2} \frac{\Gamma\left(\frac{5}{2}\right)}{\Gamma(3)}+c_{3}^{2}|A|^{4} \frac{\Gamma\left(\frac{7}{2}\right)}{\Gamma(4)}+c_{4}^{2}|A|^{6} \frac{\Gamma\left(\frac{9}{2}\right)}{\Gamma(5)}\right\}^{1 / 2} \\
& =\sqrt{c_{1}^{2}+\frac{3}{4} c_{2}^{2}|A|^{2}+\frac{5}{8} c_{3}^{2}|A|^{4}+\frac{35}{64} c_{4}^{2}|A|^{6}}
\end{aligned}
$$

que é a mesma relação de frequência-amplitude obtida por Liu (2009, página 2425).

A Figura 4 mostra a variação do erro relativo em porcentagem como uma função da amplitude para: $c_{1}^{2}=c_{2}^{2}=c_{3}^{2}=c_{4}^{2}=1$ (linha contínua) e $c_{1}^{2}=c_{2}^{2}=c_{2}^{3}=1, c_{4}^{2}=0$ (linha tracejada). Como anteriormente, o tempo total de simulação é $T$ que varia para cada valor de amplitude. É observado que a abordagem Hamiltoniana é adequada para ser aplicada para ordens de não-linearidades até aproximadamente $\alpha=5$. Esse fato também é ilustrado na Tabela 4 em que na presença de não-linearidades de ordem sétima o erro relativo converge aproximadamente para $8.7 \%$. 
Tabela 4 - Período numérico, período aproximado e erros relativos para diversos conjuntos dos parâmetros $c_{1}^{2}, c_{2}^{2}, c_{3}^{2}$, e $c_{4}^{2}$ e valor de amplitude inicial $A=40$.

\begin{tabular}{lllllll}
\hline$c_{1}^{2}$ & $c_{2}^{2}$ & $c_{3}^{2}$ & $c_{4}^{2}$ & $T_{n}$ & $T$ & Erro \% \\
\hline 1 & 1 & 1 & 1 & $1.454 \mathrm{e}-4$ & $1.327 \mathrm{e}-4$ & 8.72 \\
1 & 0 & 1 & 1 & $1.454 \mathrm{e}-4$ & $1.327 \mathrm{e}-4$ & 8.72 \\
1 & 1 & 0 & 1 & $1.454 \mathrm{e}-4$ & $1.328 \mathrm{e}-4$ & 8.73 \\
1 & 0 & 0 & 1 & $1.454 \mathrm{e}-4$ & $1.328 \mathrm{e}-4$ & 8.73 \\
1 & 1 & 1 & 0 & $5.256 \mathrm{e}-3$ & $4.965 \mathrm{e}-3$ & 5.53 \\
1 & 0 & 1 & 0 & $5.258 \mathrm{e}-3$ & $4.967 \mathrm{e}-3$ & 5.53 \\
1 & 1 & 0 & 0 & $1.853 \mathrm{e}-1$ & $1.813 \mathrm{e}-1$ & 2.17 \\
100 & 100 & 100 & 100 & $1.454 \mathrm{e}-5$ & $1.327 \mathrm{e}-5$ & 8.72 \\
100 & 0 & 100 & 100 & $1.454 \mathrm{e}-5$ & $1.327 \mathrm{e}-5$ & 8.72 \\
100 & 100 & 0 & 100 & $1.454 \mathrm{e}-5$ & $1.328 \mathrm{e}-5$ & 8.73 \\
100 & 0 & 0 & 100 & $1.454 \mathrm{e}-5$ & $1.328 \mathrm{e}-5$ & 8.73 \\
100 & 100 & 100 & 0 & $5.256 \mathrm{e}-4$ & $4.965 \mathrm{e}-4$ & 5.53 \\
100 & 0 & 100 & 0 & $5.258 \mathrm{e}-4$ & $4.967 \mathrm{e}-4$ & 5.53 \\
100 & 100 & 0 & 0 & $1.853 \mathrm{e}-2$ & $1.813 \mathrm{e}-2$ & 2.17 \\
\hline
\end{tabular}




\section{Oscilador com não-linearidades de ordens não-inteiras:}

Seja um oscilador mecânico descrito por uma equação com não-linearidades polinomial de ordem não-inteiras:

$$
\ddot{x}+c_{1}^{2} x+c_{2}^{2} x|x|^{2 / 3}+c_{3}^{2} x|x|^{4 / 3}+c_{4}^{2} x^{3}=0 .
$$

Utilizando o procedimento proposto na presente seção e a expressão (3.30)), a frequência aproximada de vibração é expressa por:

$$
\omega=\sqrt{c_{1}^{2}+0.89147 c_{2}^{2}|A|^{2 / 3}+0.81171 c_{3}^{2}|A|^{4 / 3}+0.75 c_{4}^{2} A^{2}} .
$$

O período aproximado (3.32) é comparado com o obtido numericamente chamado de período de vibração 'exato', $T_{n}$. Como nos casos anteriores esse período é calculado usando a solução numérica da equação Eq. (3.71) com as condições iniciais $x(0)=A, \dot{x}(0)=0$ por meio do método de Runge-Kutta. A Figura 5 apresenta a variação do erro relativo em porcentagem versus a amplitude de oscilação para dois conjuntos de parâmetros do sistema: $c_{1}^{2}=c_{2}^{2}=c_{3}^{2}=c_{4}^{2}=1$ (linha contínua) e $c_{1}^{2}=c_{4}^{2}=1, c_{2}^{2}=c_{3}^{2}=0$ (linha tracejada). O período de vibração é calculado numericamente no intervalo temporal de $0 \leqslant t \leqslant T$ com o incremento de tempo de $\Delta t=10^{-6} T$, onde $T$ é o período aproximado que, como nos casos anteriores, é uma função da amplitude inicial de vibração $A$ que varia no intervalo $0 \leqslant A \leqslant 40$ com incremento $\Delta A=0.1$. Quando a amplitude é igual a $A=40$, observa-se que o erro relativo converge para ambos casos dos conjuntos dos parâmetros para valores pequenos, isto é, o erro máximo do período de vibração para $c_{1}^{2}=c_{2}^{2}=c_{3}^{2}=c_{4}^{2}=1$ é aproximadamente $2.050 \%$ e para $c_{1}^{2}=c_{4}^{2}=1, c_{2}^{2}=c_{3}^{2}=0$ o erro converge para $2.169 \%$. A Figura 5 mostra que os coeficientes dos termos racionais $\left(c_{2}^{2} \mathrm{e}\right.$ $\left.c_{3}^{2}\right)$ na equação Eq. (3.71) fornecem erros relativos maiores para amplitudes inicias até $A=1.3$. Após esse ponto, a ordem de não-linearidade cúbica é dominante. Mesmo assim, para ambos conjuntos de parâmetros os erros são limitados. Pode-se inferir que o período de vibração calculado analiticamente tem alta precisão independentemente dos valores das amplitudes de vibração inciais, isto é, o erro é menor do que $2.5 \%$ mesmo quando a amplitude é igual a $A=40$.

\section{Estimativa de erro:}

Nesta seção é apresentada uma estimativa de erro da solução aproximada trigonométrica para a Eq. (3.71) com frequência representada pela Eq. (3.72). Escrevendo o Hamiltoniano para a equação Eq. (3.71) obtém-se:

$$
H=\frac{1}{2} \dot{x}^{2}+\frac{1}{2} c_{1}^{2} x^{2}+\frac{3}{8} c_{2}^{2} x^{2}|x|^{2 / 3}+\frac{3}{10} c_{3}^{2} x^{2}|x|^{4 / 3}+\frac{1}{4} c_{4}^{2} x^{4} .
$$




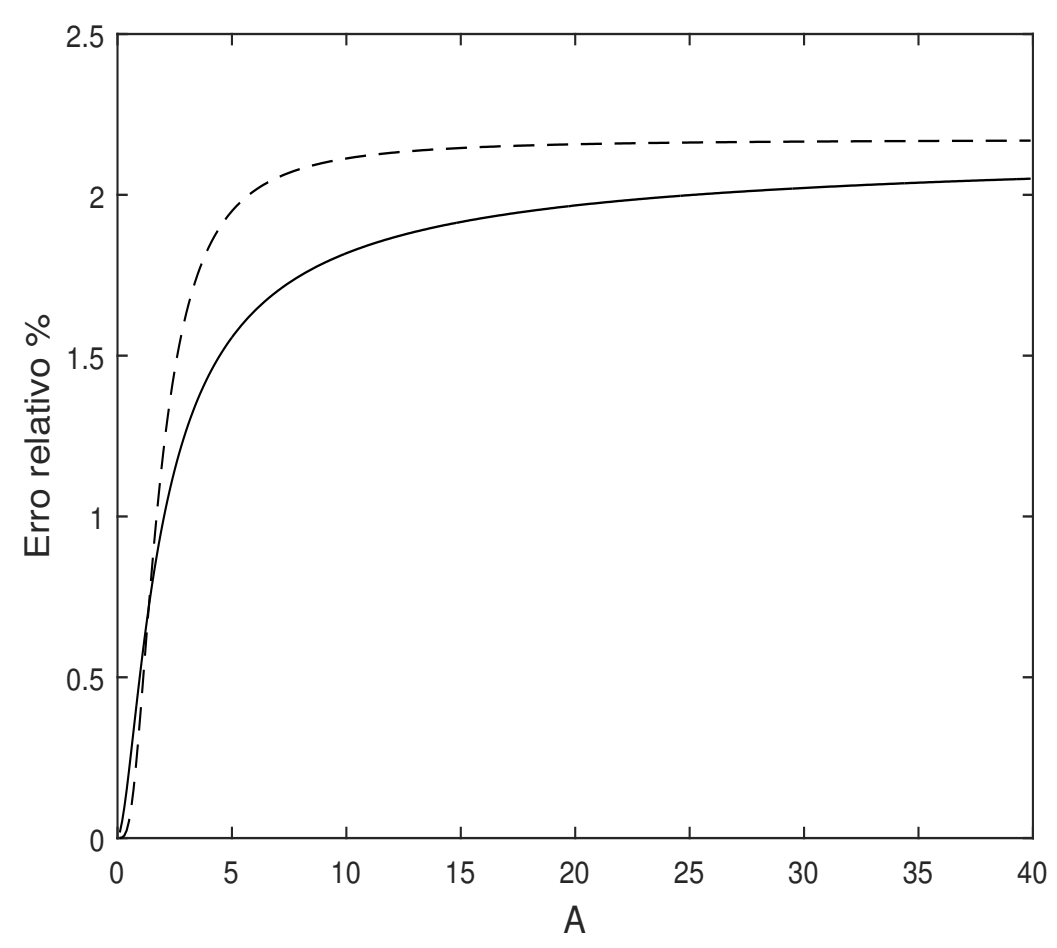

Figura 5 - Erro relativo (\%) versus amplitude, $A$, para $c_{1}^{2}=c_{2}^{2}=c_{3}^{2}=c_{4}^{2}=1$ (linha contínua) e $c_{1}^{2}=c_{4}^{2}=1, c_{2}^{2}=c_{3}^{2}=0$ (linha tracejada).

Considerando as condições iniciais $x(0)=A$ e $\dot{x}(0)=0$, a energia mecânica constante do sistema é

$$
H_{0}=\frac{1}{2} c_{1}^{2} A^{2}+\frac{3}{8} c_{2}^{2} A^{8 / 3}+\frac{3}{10} c_{3}^{2} A^{10 / 3}+\frac{1}{4} c_{4}^{2} A^{4}
$$

Substituindo a solução aproximada Eq. (3.42) na equação Eq. (3.73) e usando a relação (3.37), a função de erro de acordo com (3.39) é expressa por:

$$
\begin{aligned}
\Delta_{2}= & \frac{2}{\pi} \int_{0}^{\pi / 2}\left[\frac{1}{2} A^{2} \omega^{2} \sin ^{2}(\psi)+\frac{1}{2} c_{1}^{2} A^{2} \cos ^{2}(\psi)+\frac{3}{8} c_{2}^{2} A^{8 / 3} \cos ^{2}(\psi)|\cos (\psi)|^{2 / 3}\right. \\
& \left.+\frac{3}{10} c_{3}^{2} A^{10 / 3} \cos ^{2}(\psi)|\cos (\psi)|^{4 / 3}+\frac{1}{4} c_{4}^{2} A^{4} \cos ^{4}(\psi)-H_{0}\right]^{2} d \psi
\end{aligned}
$$

isto é,

$$
\begin{aligned}
& \Delta_{2}=\frac{2}{\pi}\left\{a_{1}^{2} S_{4}+a_{2}^{2} C_{4}+a_{3}^{2} C_{3}+2 a_{1} a_{2} S_{3}+2 a_{1} a_{3} S_{2 / 3}+2 a_{2} a_{3} C_{4 / 3}\right. \\
& +a_{4}^{2} C_{8 / 3}+a_{5}^{2} C_{8}+H_{0}^{2} \frac{\pi}{2}+2 a_{4} a_{5} C_{6 / 3}-2 H_{0} a_{4} C_{10}-2 H_{0} a_{5} C_{4} \\
& +2\left[a_{1} a_{4} C_{14}+a_{2} a_{4} C_{13}+a_{3} a_{4} C_{12}\right. \\
& +2\left[a_{1} a_{5} S_{1}+a_{2} a_{5} C_{6}+a_{3} a_{5} C_{0}\right] \\
& \left.-2\left[a_{1} H_{0} S_{2}+a_{2} H_{0} C_{2}+a_{3} H_{0} C_{2 / 3}\right]\right\}
\end{aligned}
$$

onde

$$
a_{1}=0.5 A^{2} c_{1}^{2}+0.44574 c_{2}^{2} A^{8 / 3}+0.40586 c_{3}^{2} A^{10 / 3}+0.375 c_{4}^{2} A^{4}
$$




$$
a_{2}=\frac{1}{2} c_{1}^{2} A^{2}, \quad a_{3}=\frac{3}{8} c_{2}^{2} A^{8 / 3}, \quad a_{4}=\frac{3}{10} c_{3}^{2} A^{10 / 3}, \quad a_{5}=\frac{1}{4} c_{4}^{2} A^{4},
$$

e

$$
\begin{aligned}
& S_{4}=\int_{0}^{\pi / 2} \sin ^{4}(\psi) d \psi, \quad S_{2 / 3}=\int_{0}^{\pi / 2} \sin ^{2}(\psi) \cos ^{2}(\psi)|\cos (\psi)|^{2 / 3} d \psi, \\
& C_{12}=\int_{0}^{\pi / 2} \cos ^{4}(\psi)|\cos (\psi)|^{2 / 3}|\cos (\psi)|^{4 / 3} d \psi \\
& C_{13}=\int_{0}^{\pi / 2} \cos ^{4}(\psi)|\cos (\psi)|^{4 / 3} d \psi, \quad C_{14}=\int_{0}^{\pi / 2} \sin ^{2}(\psi) \cos ^{2}(\psi)|\cos (\psi)|^{4 / 3} d \psi, \\
& C_{4}=\int_{0}^{\pi / 2} \cos ^{4}(\psi) d \psi, \quad C_{4 / 3}=\int_{0}^{\pi / 2} \cos ^{4}(\psi)|\cos (\psi)|^{2 / 3} d \psi \\
& C_{3}=\int_{0}^{\pi / 2}\left(\cos ^{2}(\psi)|\cos (\psi)|^{2 / 3}\right)^{2} d \psi, \quad C_{8 / 3}=\int_{0}^{\pi / 2}\left(\cos ^{2}(\psi)|\cos (\psi)|^{4 / 3}\right)^{2} d \psi \\
& S_{3}=\int_{0}^{\pi / 2} \sin ^{2}(\psi) \cos ^{2}(\psi) d \psi, \quad C_{8}=\int_{0}^{\pi / 2} \cos ^{8}(\psi) d \psi \\
& S_{2}=\int_{0}^{\pi / 2} \sin ^{2}(\psi) d \psi, \quad C_{6 / 3}=\int_{0}^{\pi / 2} \cos ^{6}(\psi)|\cos (\psi)|^{4 / 3} d \psi \\
& C_{2}=\int_{0}^{\pi / 2} \cos ^{2}(\psi) d \psi, \quad C_{2 / 3}=\int_{0}^{\pi / 2} \cos ^{2}(\psi)|\cos (\psi)|^{2 / 3} d \psi, \\
& S_{1}=\int_{0}^{\pi / 2} \sin ^{2}(\psi) \cos ^{4}(\psi) d \psi, \quad C_{6}=\int_{0}^{\pi / 2} \cos ^{6}(\psi) d \psi \\
& C_{0}=\int_{0}^{\pi / 2} \cos ^{6}(\psi)|\cos (\psi)|^{2 / 3} d \psi, \quad C_{10}=\int_{0}^{\pi / 2} \cos ^{2}(\psi)|\cos (\psi)|^{4 / 3} d \psi .
\end{aligned}
$$

Após alguns cálculos e aplicando as relações (3.74) e (3.76) obtém-se a expressão (3.40) para o erro relativo. A Tabela 5 contém valores do erro $\Delta$ para $c_{1}^{2}=c_{2}^{2}=c_{3}^{2}=c_{4}^{2}=1$ de diversos valores da amplitude inicial $A$. É evidente que o erro está aumentando com o aumento de $A$, entretanto para altos valores da amplitude o erro converge para uma valor constante $\Delta \approx 0.227$.

Tabela 5 - Erro relativo para $c_{1}^{2}=c_{2}^{2}=c_{3}^{2}=c_{4}^{2}=1$ e diversas amplitudes inicais $A$.

\begin{tabular}{|l|l|l|l|l|l|l|}
\hline $\mathrm{A}$ & 0.5 & 1 & 5 & 10 & 20 & 50 \\
\hline$\Delta$ & 0.05739 & 0.09579 & 0.18807 & 0.20813 & 0.21914 & 0.22652 \\
\hline
\end{tabular}

Na Tabela 6 é apresentado o erro relativo para os parâmetros $c_{1}^{2}=c_{4}^{2}=1$, $c_{2}^{2}=c_{3}^{2}=0$ e diversos valores da amplitude inicial $A$. Observa-se que aumentando a 
Tabela 6 - Erro relativo para $c_{1}^{2}=c_{4}^{2}=1, c_{2}^{2}=c_{3}^{2}=0$ e diversas amplitudes iniciais $A$.

\begin{tabular}{|l|l|l|l|l|l|l|}
\hline $\mathrm{A}$ & 0.5 & 1 & 5 & 10 & 20 & 50 \\
\hline$\Delta$ & 0.02598 & 0.07795 & 0.21653 & 0.22927 & 0.23269 & 0.23367 \\
\hline
\end{tabular}

amplitude de vibração o erro converge para um valor limite dado constante $\Delta=0.23385$ (ver Table 2).

Analisando os dados das Tabelas 5 e 6 , pode-se concluir que para $A>3$ o erro cresce rapidamente e que para $A<3$ a precisão é maior para o oscilador do tipo Duffing do que o oscilador com ordens de não-linearidades fracionais. Mesmo assim, para ambos osciladores o erro é limitado para altas amplitudes iniciais. 


\subsection{Vibração livre de osciladores conservativos com condições ini- ciais gerais}

Nesta seção a abordagem Hamiltoniana é estendida para resolver vibrações de osciladores conservativos fortemente não-lineares com condições iniciais gerais. Baseado na hipótese de que a derivada do Hamiltoniano é nula, determina-se a frequência como função da amplitude inicial, velocidade inicial e dos parâmetros do sistema. Apresenta-se também um procedimento para tratamento de erro da solução aproximada baseado na razão entre uma função residual média e a energia mecânica total constante do sistema. Na presente seção são propostos dois algoritmos computacionais quando não é possível derivar explicitamente relações para a frequência (Algoritmo 1 chamado FREQUÊNCIA) e para o erro (Algoritmo 2 chamado DELTA). A abordagem Hamiltoniana estendida é aplicada em dois casos exemplos: equação de Duffing e um oscilador conservativo não-linear puro.

Os osciladores mecânicos não-lineares conservativos tratados na presente seção têm a seguinte equação de movimento geral:

$$
\ddot{x}+f(x)=0,
$$

com as condições iniciais generalizadas:

$$
x(0)=A, \quad \dot{x}(0)=v,
$$

onde $f(x)$ é uma força conservativa que contém não-linearidades de ordem inteiras e não-inteiras.

\subsubsection{Solução aproximada}

A equação Eq. (3.77) descreve o movimento de um oscilador conservativo não-linear com energia cinética expressa por:

$$
K=\frac{\dot{x}^{2}}{2}
$$

onde a energia potencial total, $F(x)$, é:

$$
\frac{d F(x)}{d x}=f(x)
$$

A energia mecânica total do oscilador conservativo corresponde ao seu Hamiltoniano:

$$
H=\frac{1}{2} \dot{x}^{2}+F(x)
$$

Para um oscilador conservativo a energia mecânica total mantém inalterada durante o movimento, e conseqüentemente o Hamiltoniano do sistema é constante, isto é, $H=H_{0}=$ constant, e devido as condições iniciais (3.78) representa $H_{0}$ como:

$$
H_{0}=\frac{1}{2} v^{2}+F(A)
$$


No caso de osciladores não-lineares, frequentemente não é possível obter soluções analíticas para a Eq. (3.81). Nesse caso normalmente buscam-se soluções numéricas ou soluções aproximadas. Nos tipos de vibrações livres uma solução tentativa na forma trigonométrica pode ser usada para se obter soluções aproximadas:

$$
x(t)=C \cos (\omega t-\phi)
$$

onde $\omega$ é a frequência de vibração desconhecida, $C$ é a amplitude do oscilador, e $\phi$ é a fase do oscilador. Usando a Eq. (3.83) e as condições iniciais dadas pela Eq. (3.78), obtém-se o valor máximo de $x(t)$ e a fase que são expressos, respectivamente, por:

$$
C=\sqrt{A^{2}+\left(\frac{v}{\omega}\right)^{2}}
$$

e

$$
\phi=\tan ^{-1}\left(\frac{v}{\omega A}\right)
$$

Substituindo a Eq. (3.83) na Eq. (3.81), obtém-se o Hamiltoniano aproximado:

$$
\tilde{H}=\frac{1}{2} C^{2} \omega^{2} \sin ^{2}(\omega t-\phi)+F(C \cos (\omega t-\phi)) .
$$

Normalmente, a frequência desconhecida $\omega$ é determinada a partir da derivada da Eq. (3.86)

$$
\frac{\partial \tilde{H}}{\partial C}=0
$$

Entretanto, o resultado obtido não é preciso. He (2010) contornou esse problema desenvolvendo um método, descrito na Seção 3.1, denominado de abordagem Hamiltoniana. Nesse método, He (2010) introduziu uma nova função, $\bar{H}$ que tem uma forma similar a:

$$
\bar{H}=\int_{t_{1}}^{t_{1}+T} \tilde{H} d t
$$

onde $T$ é o período de vibração e $t_{1}$ é um instante inicial arbitrário de integração. Após a integração a nova função é escrita por $\bar{H}=\tilde{H} T$, isto é,

$$
\tilde{H}=\frac{\partial \bar{H}}{\partial T}
$$

De acordo com a Eq. (3.87) e com o período de vibração $T=2 \pi / \omega$, reescreve-se a igualdade como:

$$
\frac{\partial}{\partial C}\left(\frac{\partial \bar{H}}{\partial T}\right)=\frac{\partial}{\partial C}\left(\frac{\partial \bar{H}}{\partial \omega^{-1}}\right)=\frac{\partial}{\partial C}\left(\frac{\partial \bar{H}}{\partial \omega}\right)=0 .
$$

A equação algébrica Eq. (3.90) pode ser resolvida para a obtenção da frequência aproximada do oscilador não-linear conservativo. Essa frequência é função da amplitude $C$ (ver Ref. (HE, 2010)). Utilizando as relações dadas pelas Eqs. (3.84) e (3.85), pode-se expressar, quando possível, uma relação explícita para a frequência aproximada em função da amplitude 
inicial $A$, da velocidade inicial $v$, e de outros parâmetros do sistema. Para isso escreve-se a função, $\bar{H}$, como:

$$
\bar{H}(x, \dot{x})=\int_{t_{1}}^{t_{1}+T}\left(\frac{1}{2} \dot{x}^{2}+F(x)\right) d t
$$

e utilizando a função trigonométrica Eq. (3.83), a função $\bar{H}$ é reescrita como

$$
\bar{H}(C, \omega)=\int_{t_{1}}^{t_{1}+T}\left\{\frac{1}{2} C^{2} \omega^{2} \sin ^{2}(\omega t-\phi)+F(C \cos (\omega t-\phi))\right\} d t .
$$

A seguir faz algumas manipulação na função $\bar{H}$. Primeiramente substitui na Eq. (3.92) a variável $\psi=\omega t$ e o período de vibração $T=2 \pi / \omega$, e em seguida defini-se a variável adimensional $\psi^{*}=t_{1} / T$, obtendo:

$$
\bar{H}(C, \omega)=\int_{2 \pi \psi^{*}}^{2 \pi\left(1+\psi^{*}\right)}\left\{\frac{1}{2} C^{2} \omega \sin ^{2}(\psi-\phi)+\omega^{-1} F(C \cos (\psi-\phi))\right\} d \psi
$$

onde $\psi^{*}$ é um valor arbitrário que para evitar erros numéricos é conveniente utilizar $\psi^{*}=n / m$, com $n \in \mathbb{N}$ e $m \in \mathbb{N}^{*}$. A escolha trivial para $\psi^{*}$ é $\psi^{*}=0\left(t_{1}=0\right)$ tal que a Eq. (3.93) transforma em

$$
\bar{H}(C, \omega)=\int_{0}^{2 \pi}\left\{\frac{1}{2} C^{2} \omega \sin ^{2}(\psi-\phi)+\omega^{-1} F(C \cos (\psi-\phi))\right\} d \psi .
$$

As Eqs. (3.90) e (3.94) são utilizadas em conjunto para determinar uma relação aproximada da frequência do oscilador em função da amplitude $C$.

Pode-se observar que a Eq. (3.83) tem valores máximos quando $\omega t-\phi=2 \pi n$, onde $n=0, \pm 1, \pm 2, \ldots$ O valor máximo para $n=0$ ocorre em $t=\phi / \omega$. Adotando um instante inicial $t_{1}=\phi / \omega$ e substituindo a variável $\tau=t-t_{1}$ na Eq. (3.92), reescreve-se essa equação como:

$$
\bar{H}(C, \omega)=\int_{0}^{T}\left\{\frac{1}{2} C^{2} \omega^{2} \sin ^{2}(\omega \tau)+F(C \cos (\omega \tau))\right\} d \tau .
$$

Da mesma forma que foi realizado anteriormente, isto é, substituindo na Eq. (3.95) as variáveis $\psi=\omega \tau$ e $T=2 \pi / \omega$, obtém-se:

$$
\bar{H}(C, \omega)=\int_{0}^{2 \pi}\left\{\frac{1}{2} C^{2} \omega \sin ^{2}(\psi)+\omega^{-1} F(C \cos (\psi))\right\} d \psi .
$$

Pode-se notar que a Eq. (3.96) é a expressão usada para determinar a relação entre a frequência e a amplitude com condições iniciais particulares, isto é, $x(0)=A, \dot{x}(0)=0$ descrita em vários trabalhos da literatura (por exemplo, (HE, 2010; AKBARZADE; KARGAR, 2011a; AKBARZADE; KARGAR, 2011b; HE; ZHONG; L., 2010; BAYAT; PAKAR; CVETICANIN, 2014; CVETICANIN et al., 2010; CVETICANIN; KALAMIYAZDI; ASKARI, 2012; YILDIRIM; SAADATNIA; ASKARI, 2011; BELENDEZ et al., 2011; XU; HE, 2010; YILDIRIM et al., 2011; SADEGHZADEH; KABIRI, 2016; AKBARZADE; KHAN, 2012; DURMAZ; DEMIRBAG; KAYA, 2010; YILDIRIM et al., 
2011; DURMAZ; DEMIRBAG; KAYA, 2012; KHAN; JAMIL; ARA, 2011) ). Para essas condições iniciais particulares têm-se na Eq. (3.96) $C=A$ e $\phi=0$.

Comparando a Eq. (3.94) com a Eq. (3.96), observa-se que ambas podem ser utilizadas em conjunto com a Eq. (3.90) para determinar uma relação para a frequência aproximada em função da amplitude $C$ e outros parâmetros do sistema. É recomendável o uso da Eq. (3.96) pelas seguintes razões: (a) essa expressão é mais simples e (b) é mais fácil determinar a variação do sinal da função potencial $F$ com a variação dos limites de integração, por exemplo, de 0 até $\pi$ ou de 0 até $\pi / 2$. Em alguns casos é possível derivar uma relação explícita para a frequência aproximada em função da amplitude inicial $A$, da velocidade inicial $v$ e dos parâmetros do sistema utilizando a frequência em função de $C$ e a Eq.(3.84). Quando não é possível derivar uma relação explícita para a frequência, essa pode ser determinada numericamente (para valores específicos de $A$ e $v$ ) usando o algoritmo proposto e apresentado como Algoritmo 1 (FREQUÊNCIA), onde cs são parâmetros genéricos do sistema. Após o cálculo das variáveis $\omega$ e $C$, a fase $\phi$ é determinada por meio da Eq. (3.85).

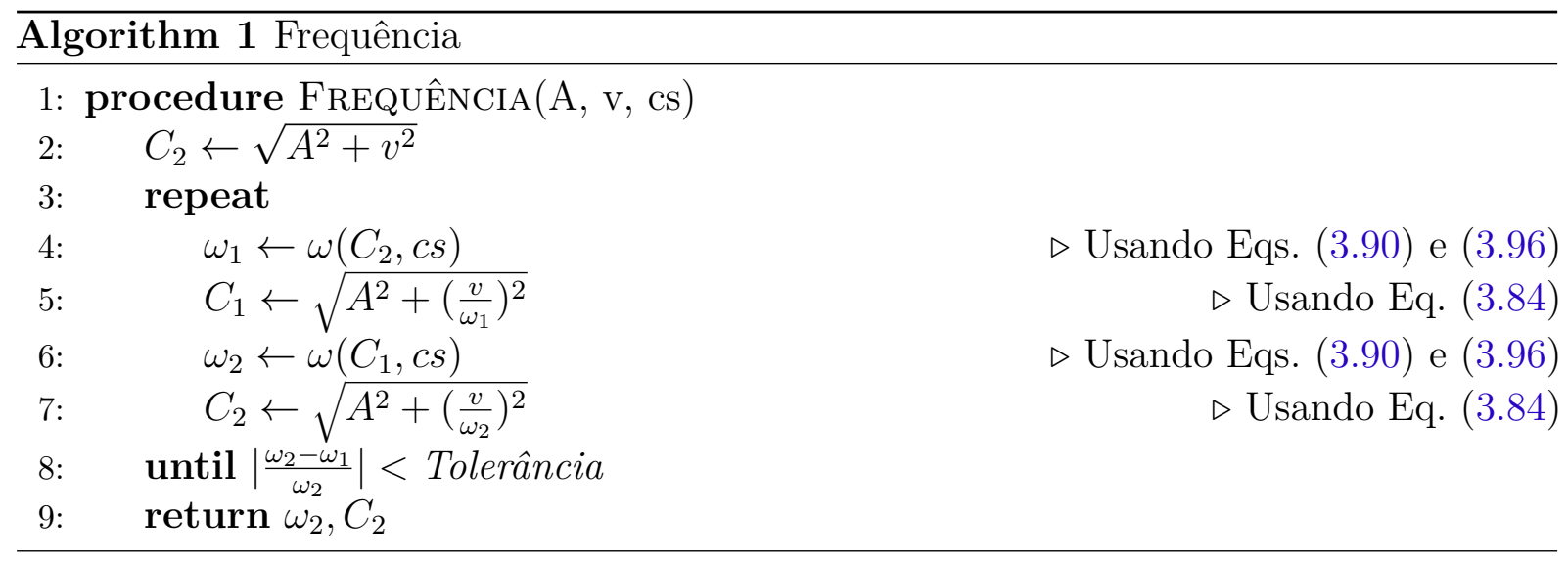

A solução aproximada, Eq. (3.83), também pode ser representada por:

$$
x(t)=C \sin \left(\omega t+\phi_{1}\right)
$$

onde a fase é dada por $\phi_{1}=\pi / 2-\phi$ ou alternativamente por:

$$
\phi_{1}=\tan ^{-1}\left(\frac{\omega A}{v}\right)
$$

\subsubsection{Estimativa de erro da solução}

Nesta seção descreve-se uma estimativa do erro da solução aproximada. A estimativa é baseada em um erro médio durante um período de vibração. Dessa forma, calcula-se o Hamiltoniano para a solução aproximada, Eq. (3.83), que difere da energia mecânica total $H_{0}$. A diferença entre $\tilde{H}$ e $H_{0}$ fornece o resíduo instantâneo expresso por:

$$
R(t)=\tilde{H}-H_{0}=\frac{1}{2} C^{2} \omega^{2} \sin ^{2}(\omega t-\phi)+F(C \cos (\omega t-\phi))-\left(\frac{1}{2} \dot{v}^{2}+F(A)\right) .
$$


Como o resíduo varia com o tempo, para determinar um valor médio, o erro é estimado como a integral o quadrado do resíduo sobre o período de vibração. Assim, para essa estimativa, define-se o seguinte funcional:

$$
\Delta_{2}=\frac{1}{T} \int_{t_{1}}^{t_{1}+T} R(t)^{2} d t=\frac{1}{T} \int_{t_{1}}^{t_{1}+T}\left(\tilde{H}-H_{0}\right)^{2} d t
$$

Substituindo a Eq. (3.99) na Eq. (3.100) obtém-se:

$$
\Delta_{2}=\frac{1}{T} \int_{t_{1}}^{t_{1}+T}\left[\frac{1}{2} C^{2} \omega^{2} \sin ^{2}(\omega t-\phi)+F(C \cos (\omega t-\phi))-\left(\frac{1}{2} \dot{v}^{2}+F(A)\right)\right]^{2} d t
$$

em seguida usando $t_{1}=\phi / \omega$ e $\tau=t-t_{1}$ tem-se:

$$
\Delta_{2}=\frac{1}{T} \int_{0}^{T}\left[\frac{1}{2} C^{2} \omega^{2} \sin ^{2}(\omega \tau)+F(C \cos (\omega \tau))-\left(\frac{1}{2} \dot{v}^{2}+F(A)\right)\right]^{2} d \tau,
$$

e para $\psi=\omega \tau, T=2 \pi / \omega$, e Eq.(3.84), o resíduo médio é escrito como:

$$
\begin{aligned}
\Delta_{2}= & \frac{1}{2 \pi} \int_{0}^{2 \pi}\left[\frac{1}{2}\left(A^{2} \omega^{2}+v^{2}\right) \sin ^{2}(\psi)+F\left(\frac{1}{\omega} \sqrt{A^{2} \omega^{2}+v^{2}} \cos (\psi)\right)\right. \\
& \left.-\left(\frac{1}{2} \dot{v}^{2}+F(A)\right)\right]^{2} d \psi
\end{aligned}
$$

A solução da Eq. (3.103) é função da amplitude inicial, velocidade inicial e dos parâmetros do sistema, isto é, $\Delta_{2}=\Delta_{2}\left(A, v, c_{k}\right)$. O erro relativo é calculado como a razão entre a raiz quadrada da função residual média e a função que representa e energia mecânica (constate) do sistema, $H_{0}$ :

$$
\Delta=\frac{\sqrt{\Delta_{2}}}{H_{0}}
$$

É possível determinar o erro relativo na forma de porcentagem $\Delta_{\%}=\left(\frac{\sqrt{\Delta_{2}}}{H_{0}}\right) 100 \%$. Quando não é possível determinar uma relação explícita para a frequência de vibração para ser usada na Eq. (3.103), o erro relativo médio, $\Delta$, pode ser determinado numericamente pelo Algoritmo 2 (DELTA), onde cs expressam os parâmetros genéricos do oscilador mecânico. O Algoritmo 2 chama o Algoritmo 1 e utiliza a integral dada pela Eq. (3.102).

\subsubsection{Comparação entre as soluções aproximadas e numéricas}

Nesta seção são apresentados os cálculos da frequência aproximada, do erro relativo médio e do erro no período vibração para a equação de Duffing e para um oscilador conservativo não-linear puro. 


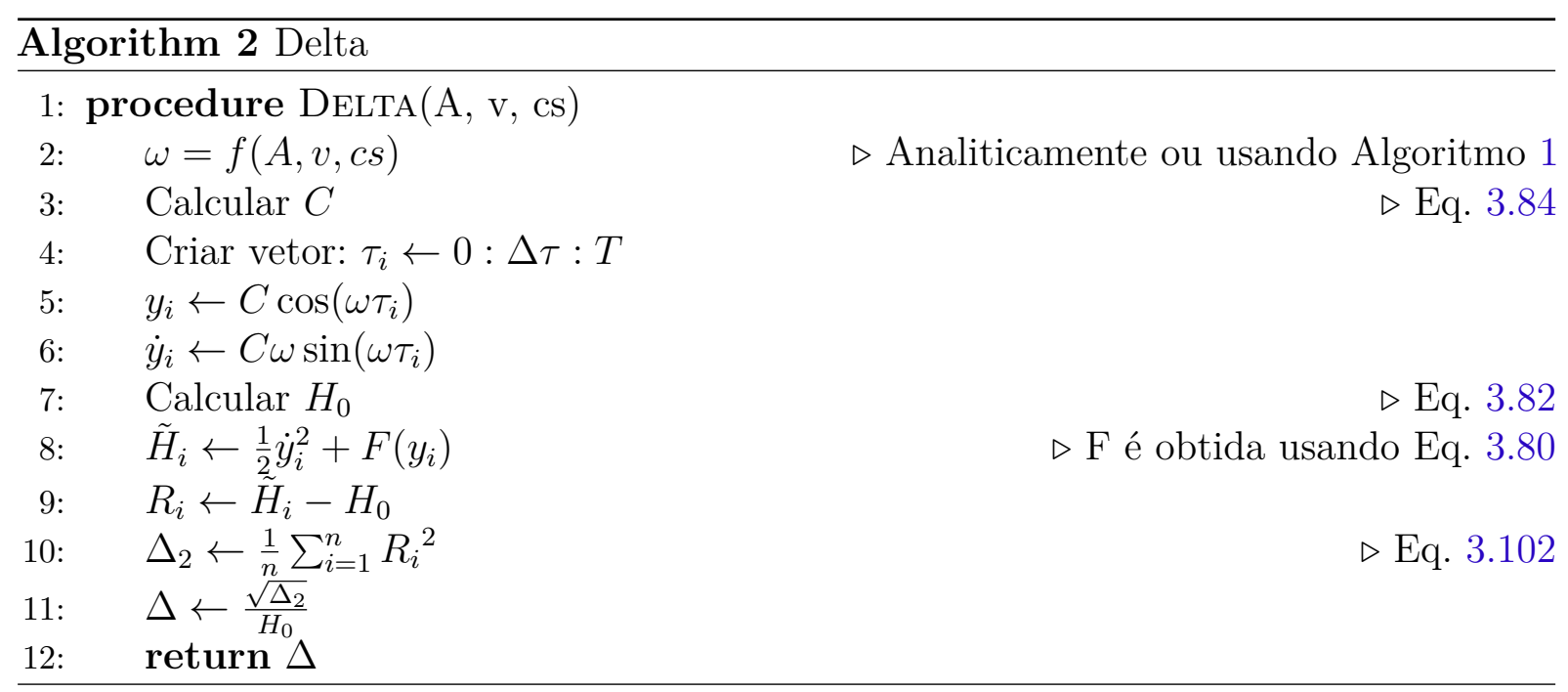

\subsubsection{Equação de Duffing}

Nesta seção é analisado um oscilador mecânico representado pela equação de Duffing

$$
\ddot{x}+c_{1}^{2} x+c_{2}^{2} x^{3}=0,
$$

com condições iniciais (3.78). Para esse oscilador é possível obter soluções explícitas para a frequência de oscilação e para o erro relativo médio sem a necessidade da utilização dos algoritmos 1 e 2. A energia potencial do oscilador de Duffing é representada por:

$$
F(x)=\frac{1}{2} c_{1}^{2} x^{2}+\frac{1}{4} c_{2}^{2} x^{4},
$$

e o Hamiltoniano do oscilador é expresso por

$$
H=\frac{1}{2} \dot{x}^{2}+\frac{1}{2} c_{1}^{2} x^{2}+\frac{1}{4} c_{2}^{2} x^{4} .
$$

Considerando uma solução aproximada escrita na forma da Eq. (3.83) a função (3.96) é representada por:

$$
\begin{aligned}
\bar{H}(C, \omega) & =4 \int_{0}^{\pi / 2}\left\{\frac{1}{2} C^{2} \omega \sin ^{2}(\psi)+\omega^{-1}\left(\frac{1}{2} c_{1}^{2} C^{2} \cos ^{2}(\psi)+\frac{1}{4} c_{2}^{2} C^{4} \cos ^{4}(\psi)\right)\right\} d \psi \\
& =\frac{C^{2} \pi\left(8 c_{1}^{2}+3 C^{2} c_{2}^{2}+8 \omega^{2}\right)}{16 \omega}
\end{aligned}
$$

e calculando as derivadas da Eq. (3.90), isto é,

$$
\frac{\partial}{\partial C}\left(\frac{\partial \bar{H}}{\partial \omega}\right)=2 \pi C-\frac{3 \pi c_{2}^{2} C^{3}}{8 \omega^{2}}-\frac{\pi C\left(8 c_{1}^{2}+3 c_{2}^{2} C^{2}+8 \omega^{2}\right)}{8 \omega^{2}}=0,
$$

a relação aproximada da frequência em função da amplitude máxima é dada por:

$$
\omega=\sqrt{c_{1}^{2}+\frac{3}{4} c_{2}^{2} C^{2}} .
$$

Como mencionado, para a equação de Duffing pode-ser escrever uma relação explícita para a frequência aproximada em função da amplitude inicial $A$, da velocidade inicial $v$, 
e dos parâmetros $c_{1}$ e $c_{2}$. Dessa forma, utilizando as Eqs. (3.84) e (3.110), a frequência aproximada é dada por:

$$
\omega=\sqrt{\frac{c_{1}^{2}}{2}+\frac{3}{8} c_{2}^{2} A^{2}+\sqrt{\left(\frac{c_{1}^{2}}{2}+\frac{3}{8} c_{2}^{2} A^{2}\right)^{2}+\frac{3}{4} c_{2}^{2} v^{2}},}
$$

e de acordo com a Eq. (3.85) a fase é calculada usando a expressão:

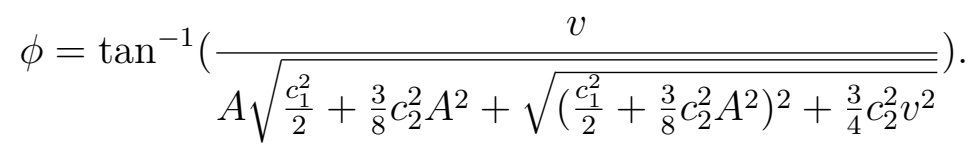

Considerando o caso em que a velocidade inicial é nula, $v=0$, a frequência aproximada e a fase são expressas, respectivamente por:

$$
\begin{aligned}
\omega & =\sqrt{c_{1}^{2}+\frac{3}{4} c_{2}^{2} A^{2}} \\
\phi & =0
\end{aligned}
$$

e para o caso de impacto onde $A=0$ com $v \neq 0$, tem-se:

$$
\begin{aligned}
& \omega=\frac{\sqrt{2}}{2} \sqrt{c_{1}^{2}+\sqrt{c_{1}^{4}+3 c_{2}^{2} v^{2}}} \\
& \phi=\frac{\pi}{2} .
\end{aligned}
$$

Assim, o período aproximada de oscilação é calculado por:

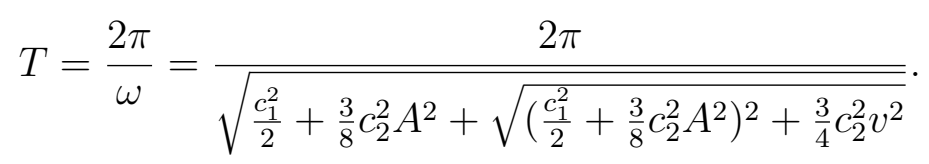

A seguir é comparado o período aproximada dado pela Eq. (3.115) com o obtido numericamente. O período de vibração numérico ('exato'), $T_{n}$, é calculado usando a solução numérica da Eq. (3.105) com as condições iniciais (3.78) pelo método de Runge-Kutta. O histórico no tempo da solução é determinado durante o intervalo $0 \leqslant t \leqslant T$ com incremento temporal de $\Delta t$, onde $T$ é o período aproximado de vibração, Eq. (3.115). Com o histórico no tempo determina-se o período numérico de vibração, sendo que o erro relativo em percentagem é dado por:

$$
\Delta_{T}=\left|\frac{T_{n}-T}{T_{n}}\right| \cdot 100 \%
$$

A Figura 6 ilustra a variação do erro relativo em porcentagem versus a amplitude inicial, $A$, para três valores diferentes da velocidade inicial, isto é, $v=0$ (linha contínua), $v=10$ (linha tracejada) e $v=100$ (linha pontilhada). Na construção da Figura 6 utilizou-se os parâmetros do sistema $c_{1}^{2}=c_{2}^{2}=1$ e a amplitude inicial de vibração variou no intervalo $0 \leqslant A \leqslant 50$ com incremento $\Delta A=0.5$. Na solução numérica da equação de Duffing utilizou-se o incremento temporal de $\Delta t=10^{-5} \mathrm{~T}$. Pode-se observar que para $v=0$ o erro relativo tem um crescimento monotônico, enquanto para $v=10$ e $v=100$ o erro relativo 


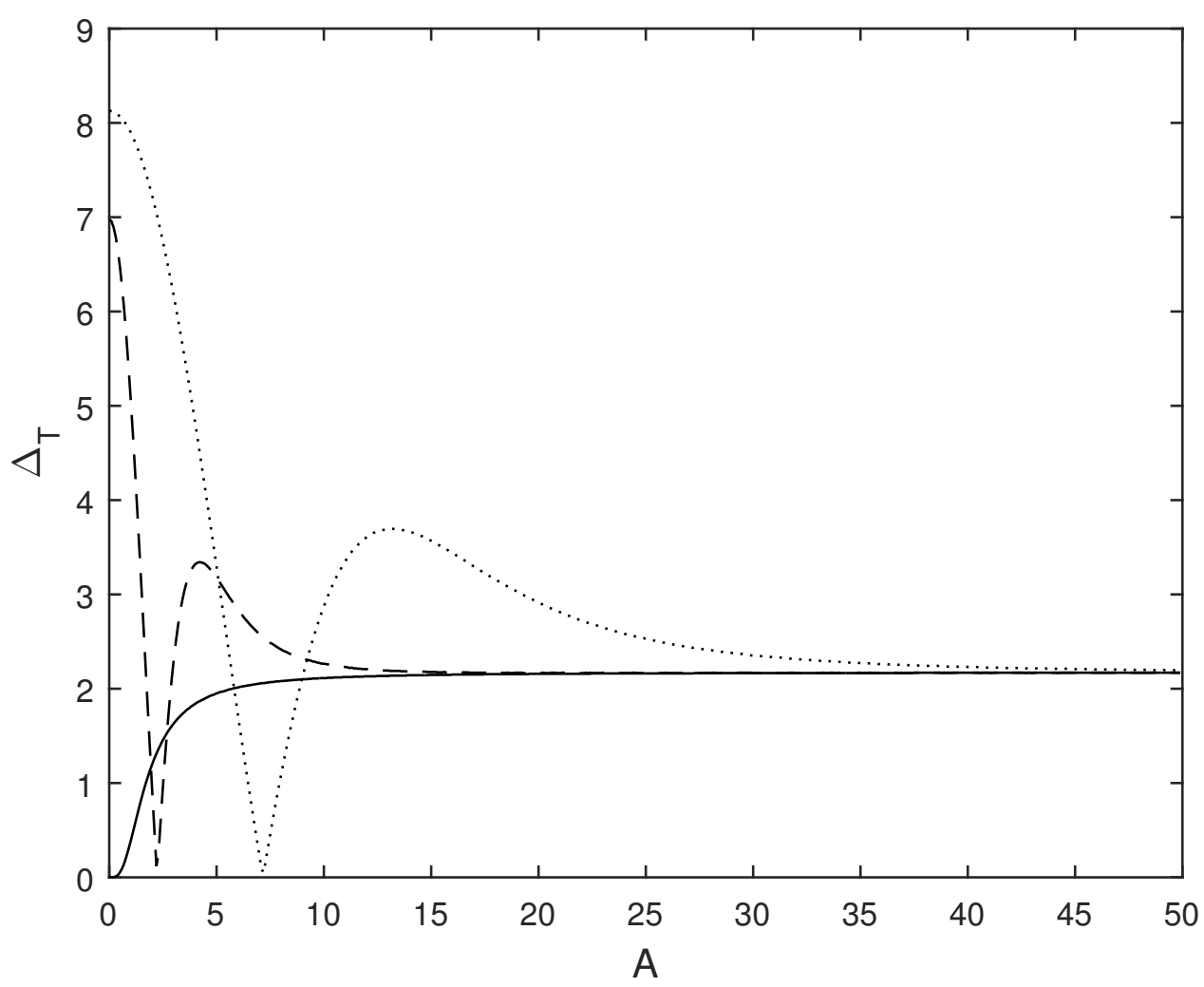

Figura 6 - Erros relativos, $\Delta_{T}$ (Eq. 3.116), versus amplitude, $A$, para $v=0$ (linha contínua), $v=10$ (linha tracejada) e $v=100$ (linha pontilhada).

começa em um valor alto e depois se estabiliza com o crescimento de $A$. Para os três valores da velocidade inicial o erro relativo do período converge para $A=50$. Conclui-se que o erro relativo é sensível a velocidade inicial para pequenos valores da amplitude inicial e que essa sensibilidade aumenta com o valor de $v$.

A Tabela 7 mostra valores do erro relativo do período em porcentagem, Eq. (3.116), para os parâmetros $c_{1}^{2}=c_{2}^{2}=1$ e para diversos valores das condições iniciais, isto é, amplitude $A$ e velocidade $v$. Para o cálculo dos valores da Tabela 7 utilizou-se o incremento temporal de $\Delta t=10^{-7} T$. Todos os números da Tabela 7 foram arredondados para três casas decimais. O mesmo comportamento obtido na Figura 6 é mostrado na Tabela 7. Podemos notar, também, na Tabela 7 um crescimento monotônico para $v=0$ e para valores de velocidade $v>0$, o erro relativo começa em um valor alto para $A=0$ e com o aumento do valor de $A$, o erro relativo decresce até um valor mínimo. Após esse ponto, o erro relativo começa aumentar convergindo para o valor $2.172 \%(A \rightarrow \infty)$, independente do valor de $v$. Observa-se também para altos valores de velocidade $v$ com amplitude inicial nula $A=0$, o erro relativo é maior e esse erro converge para $8.264 \%$ quando $v$ aumenta, isto é, $(A=0, v \rightarrow \infty)$.

A seguir determina-se o erro médio entre as soluções aproximada e exata. Conside- 
Tabela 7 - Erros relativos, $\Delta_{T}$ (Eq. 3.116), para $c_{1}^{2}=c_{2}^{2}=1$ e diversas condições iniciais $A, v$.

\begin{tabular}{|c|c|c|c|c|c|c|c|c|}
\hline & 0 & 1 & 5 & 10 & 50 & 100 & 500 & 1000 \\
\hline 0 & - & 0.385 & 1.949 & 2.113 & 2.170 & 2.172 & 2.172 & 2.172 \\
\hline 1 & 1.354 & 1.010 & 1.972 & 2.115 & 2.170 & 2.172 & 2.172 & 2.172 \\
\hline 5 & 5.861 & 2.807 & 2.431 & 2.152 & 2.170 & 2.172 & 2.172 & 2.172 \\
\hline 10 & 6.977 & 5.070 & 3.179 & 2.263 & 2.170 & 2.172 & 2.172 & 2.172 \\
\hline 50 & 7.993 & 7.542 & 0.106 & 3.615 & 2.176 & 2.172 & 2.172 & 2.172 \\
\hline 100 & 8.127 & 7.898 & 3.294 & 2.873 & 2.196 & 2.173 & 2.172 & 2.172 \\
\hline 500 & 8.237 & 8.190 & 7.108 & 4.180 & 2.707 & 2.212 & 2.172 & 2.172 \\
\hline 1000 & 8.250 & 8.227 & 7.675 & 6.067 & 3.494 & 2.327 & 2.172 & 2.172 \\
\hline
\end{tabular}

rando as condições iniciais $x(0)=A$ e $\dot{x}(0)=v$, o Hamiltoniano do sistema é dado por:

$$
H_{0}=\frac{1}{2} v^{2}+\frac{1}{2} c_{1}^{2} A^{2}+\frac{1}{4} c_{2}^{2} A^{4}
$$

Substituindo a solução trigonométrica aproximada Eq. (3.83) na Eq. (3.107) e usando as relações dadas pelas Eqs. (3.100) e (3.117), a função do erro, Eq. (3.103), é expressa por:

$$
\begin{aligned}
\Delta_{2}= & \frac{2}{\pi} \int_{0}^{\pi / 2}\left[\frac{1}{2} C^{2} \omega^{2} \sin ^{2}(\psi)+\frac{1}{2} c_{1}^{2} C^{2} \cos ^{2}(\psi)+\frac{1}{4} c_{2}^{2} C^{4} \cos ^{4}(\psi)\right. \\
& \left.-\left(\frac{1}{2} v^{2}+\frac{1}{2} c_{1}^{2} A^{2}+\frac{1}{4} c_{2}^{2} A^{4}\right)\right]^{2} d \psi
\end{aligned}
$$

Utilizando as equações para a amplitude e frequência, Eqs.(3.84) e (3.111), respectivamente, e fazendo alguns cálculos, a função $\Delta_{2}$ é reescrita como:

$$
\begin{aligned}
\Delta_{2}= & \frac{A^{4} c_{1}^{4}}{6}+\frac{23 c_{1}^{8}}{1296 c_{2}^{4}}+\frac{A^{2} c_{1}^{6}}{12 c_{2}^{2}}+\frac{1}{8} A^{6} c_{1}^{2} c_{2}^{2}+\frac{135 A^{8} c_{2}^{4}}{4096} \\
& +\frac{85}{576} A^{2} c_{1}^{2} v^{2}+\frac{23 c_{1}^{4} v^{2}}{432 c_{2}^{2}}+\frac{107}{768} A^{4} c_{2}^{2} v^{2}+\frac{23 v^{4}}{1152} \\
& -\frac{263 A^{4} c_{1}^{2} R}{9216}-\frac{23 c_{1}^{6} R}{5184 c_{2}^{4}}-\frac{121 A^{2} c_{1}^{4} R}{6912 c_{2}^{2}}-\frac{121 A^{6} c_{2}^{2} R}{12288} \\
& -\frac{49 A^{2} v^{2} R}{4608}-\frac{23 c_{1}^{2} v^{2} R}{3456 c_{2}^{2}},
\end{aligned}
$$

onde

$$
R=\sqrt{16 c_{1}^{4}+24 A^{2} c_{1}^{2} c_{2}^{2}+9 A^{4} c_{2}^{4}+48 c_{2}^{2} v^{2}},
$$

e de acordo com as Eqs. (3.104) e (3.117), o erro relativo médio, $\Delta$, é dado por

$$
\Delta=\frac{\sqrt{\Delta_{2}}}{\frac{1}{2} v^{2}+\frac{1}{2} c_{1}^{2} A^{2}+\frac{1}{4} c_{2}^{2} A^{4}} .
$$

Como no caso do cálculo do erro relativo do período, a Figura 7 ilustra os erros relativos médios, $\Delta$ (Eq. 3.121), versus a amplitude inicial na faixa $0 \leqslant A \leqslant 50(\Delta A=0.1)$ 


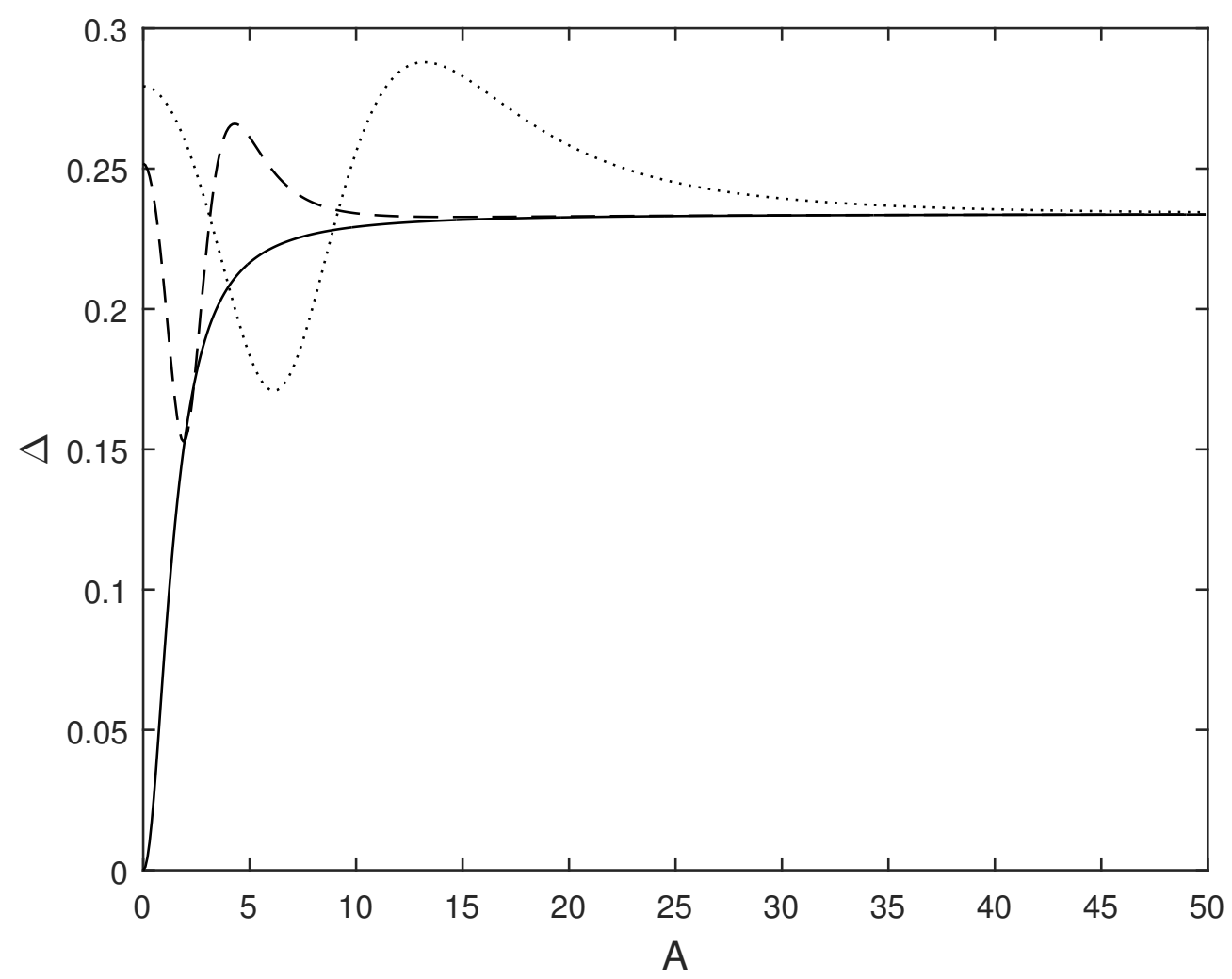

Figura 7 - Erros relativos, $\Delta$ (Eq. 3.121), versus amplitude, $A$, para $v=0$ (linha contínua), $v=10$ (linha tracejada) e $v=100$ (linha pontilhada).

para três valores da velocidade inicial, isto é, $v=0$ (linha contínua), $v=10$ (linha tracejada) e $v=100$ (linha pontilhada), com $c_{1}^{2}=c_{2}^{2}=1$. Observa o mesmo padrão encontrado na Figura 6 para o cálculo do erro relativo médio. A diferença entre os dois casos é que o erro relativo médio envolve uma faixa temporal e dessa forma os erros são maiores do que o caso do erro relativo para um determinado período.

\subsubsection{Oscilador conservativo não-linear puro}

Considerando um oscilador conservativo não-linear puro que apresenta a seguinte equação diferencial:

$$
\ddot{x}+c_{1}^{2} x|x|^{\alpha-1}=0 .
$$

De acordo com a Eq. (3.83), a solução aproximada é escrita como:

$$
x(t)=C \cos (\omega t-\phi),
$$

e usando as Eqs. (3.90) e (3.96), a frequência do oscilador é expressa por:

$$
\omega^{2}=c_{1}^{2}|C|^{\alpha-1} K_{\alpha}
$$

$\mathrm{com}$

$$
K_{\alpha}=\frac{2}{\pi} \mathrm{B}\left(\frac{1}{2}, \frac{\alpha+2}{2}\right)
$$


onde B é a função beta de Euler. Se a velocidade é nula, $v=0$, a frequência e fase aproximadas são representadas, respectivamente, por:

$$
\begin{aligned}
& \omega=\sqrt{c_{1}^{2}|A|^{\alpha-1} K_{\alpha}} \\
& \phi=0
\end{aligned}
$$

para a amplitude inicial nula, $A=0$

$$
\begin{aligned}
\omega & =\left(c_{1}^{2}|v|^{\alpha-1} K_{\alpha}\right)^{\frac{1}{\alpha+1}} \\
\phi & =\frac{\pi}{2}
\end{aligned}
$$

e para valores gerais $A$ e $v$ a frequência do oscilador é calculada usando a Eq. (3.124) e o Algoritmo 1. Com esse valor de frequência, a amplitude máxima $C$ e a fase $\phi$ são determinadas pelas Eqs. (3.84) e (3.85), respectivamente. O período aproximada de oscilação é dado por $T=2 \pi / \omega$, onde a frequência também é determinada pelo Algoritmo 1. O período aproximado, $T$, é comparado com o período 'exato' , $T_{n}$, que é obtido numericamente resolvendo a Eq. (3.122) com as condições iniciais (3.78) pelo método de Runge-Kutta no intervalo temporal $0 \leqslant t \leqslant T$ com incremento $\Delta t=10^{-7} T$. O erro relativo do período, $\Delta_{T}$, é calculado usando a Eq.(3.116). O erro médio relativo, $\Delta$, é calculado pelo Algoritmo 2. A Tabela 8 apresenta valores dos erros relativos $\Delta_{T}$ e $\Delta$ para diversos valores de $\alpha$ e para o parâmetro do sistema $c_{1}^{2}=1$. Quando a velocidade inicial é nula, $v=0$, os erros $\Delta_{T}$ e $\Delta$, são independentes da amplitude inicial $A$ e da constante $c_{1}^{2}$. Observa-se na Tabela 8 que para $\alpha<1$ os erros decrescem com o aumento de $\alpha$ e que para $\alpha>1$ ocorre o contrário, isto é, os erros aumentam com $\alpha$. Para o caso linear, $\alpha>1$, a solução aproximada corresponde a solução exata, $x(t)=C \cos \left(\left|c_{1}\right| t-\phi\right)$, e longe desse valor ( $\alpha=0$ ou $\alpha=3$ ) o erro relativo aumenta devido aos efeitos não-lineares. Navarro e Cveticanin (2016) estudaram as soluções quando $v=0$ e mostram que para altos valores de $\alpha$ o erro relativo diverge. Nota-se também que as seguintes igualdades são válidas para um valor específico de $\alpha: \Delta_{T}(A, 0)=\Delta_{T}(A \rightarrow \infty, v)$ e $\Delta(A, 0)=\Delta(A \rightarrow \infty, v)$. Na Tabela 8 essas igualdades são observadas para a velocidade inicial $v=10$.

As Figuras 8 e 9 mostram, respectivamente, os erros relativos do período, $\Delta_{T}$, e os erros médios relativos, $\Delta$ (calculado pelo Algoritmo 2), em função da amplitude inicial $0 \leqslant A \leqslant 50(\Delta A=0.1)$ e para a velocidade inicial $v=10$ e o parâmetro do sistema $c_{1}^{2}=1$. As Figuras 8 e 9 ilustram três curvas de erros de acordo com a potência $\alpha$, isto é, $\alpha=1 / 2$ (linha contínua), $\alpha=3 / 2$ (linha tracejada) e $\alpha=3$ (linha pontilhada). Observa-se que em ambas as figuras os erros começam em um valor alto e então oscilam convergindo com o aumento da amplitude inicial $(A=50)$. Os valores mínimos dos erros relativos ocorrem para valores menores de $A$ quando a potência $\alpha$ é aumentada. 
Tabela 8 - Valores dos erros relativos, $\Delta_{T}(A, v)$ e $\Delta(A, v)$, para diversos valores da potência $\alpha$.

\begin{tabular}{|l|l|l|l|l|l|l|l|}
\hline$\alpha$ & 0 & $1 / 2$ & 1 & $3 / 2$ & 2 & $5 / 2$ & 3 \\
\hline$\Delta_{T}(A, 0)$ & 1.5649 & 0.3103 & 0 & 0.2117 & 0.7203 & 1.3987 & 2.1723 \\
\hline$\Delta_{(A, 0)}$ & 0.11096 & 0.05957 & 0 & 0.06079 & 0.12047 & 0.17823 & 0.23385 \\
\hline$\Delta_{T}(0,10)$ & 23.370 & 2.7389 & 0 & 1.1411 & 3.3583 & 5.8275 & 8.2642 \\
\hline$\Delta_{(0,10)}$ & 0.52475 & 0.17855 & 0 & 0.11022 & 0.18556 & 0.24056 & 0.28260 \\
\hline$\Delta_{T}(1,10)$ & 22.164 & 2.5547 & 0 & 0.9867 & 2.7627 & 4.5399 & 6.0783 \\
\hline$\Delta_{(1,10)}$ & 0.49605 & 0.16669 & 0 & 0.09817 & 0.16116 & 0.20409 & 0.2379 \\
\hline$\Delta_{T}(5,10)$ & 17.866 & 1.3809 & 0 & 0.1548 & 1.1174 & 2.4310 & 3.4975 \\
\hline$\Delta_{(5,10)}$ & 0.39674 & 0.09721 & 0 & 0.05788 & 0.13734 & 0.21773 & 0.28097 \\
\hline$\Delta_{T}(10,10)$ & 13.516 & 0.3133 & 0 & 0.3714 & 1.0361 & 1.6631 & 2.3273 \\
\hline$\Delta_{(10,10)}$ & 0.30175 & 0.05655 & 0 & 0.07108 & 0.13361 & 0.18736 & 0.23881 \\
\hline$\Delta_{T}(20,10)$ & 7.4054 & 0.4490 & 0 & 0.2667 & 0.7731 & 1.4251 & 2.1824 \\
\hline$\Delta_{(20,10)}$ & 0.18253 & 0.06318 & 0 & 0.06402 & 0.12251 & 0.17910 & 0.23417 \\
\hline$\Delta_{T}(50,10)$ & 0.1732 & 0.5300 & 0 & 0.2181 & 0.7238 & 1.3998 & 2.1725 \\
\hline$\left.\Delta_{(50,10)}(A) \infty, 10\right)$ & 0.10583 & 0.06565 & 0 & 0.06115 & 0.12060 & 0.17826 & 0.23386 \\
\hline$\Delta_{T}(A \rightarrow \infty 949$ & 0.3103 & 0 & 0.2117 & 0.7203 & 1.3987 & 2.1723 \\
\hline$\Delta_{(A \rightarrow \infty, 10)}(A \rightarrow \infty)$ & 0.11096 & 0.05957 & 0 & 0.06079 & 0.12047 & 0.17823 & 0.23385 \\
\hline
\end{tabular}

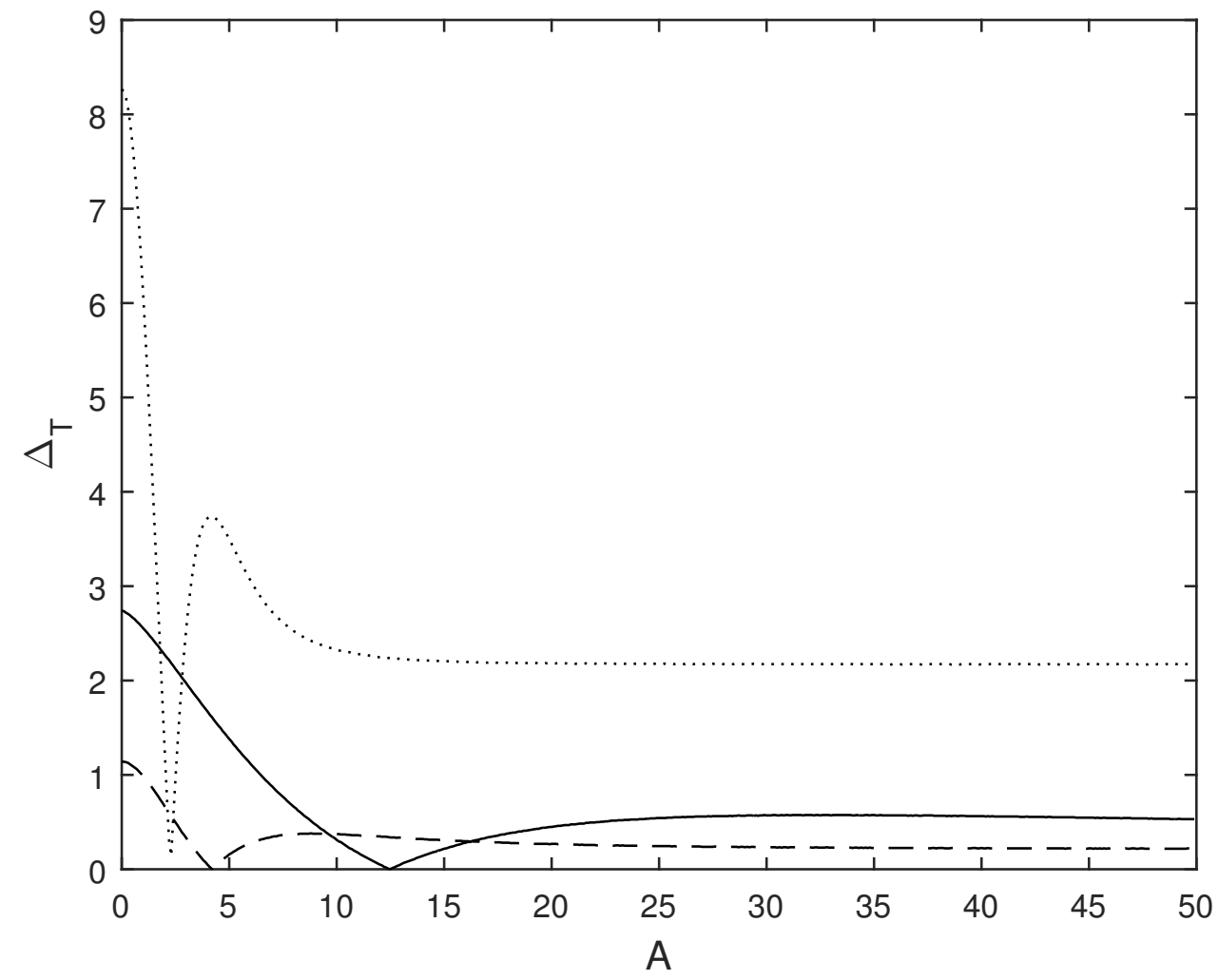

Figura 8 - Erros relativos, $\Delta_{T}$ (Eq. 3.116), versus amplitude, $A$, para $v=10, \alpha=1 / 2$ (linha contínua), $\alpha=3 / 2$ (Linha tracejada) e $\alpha=3$ (linha pontilhada). 


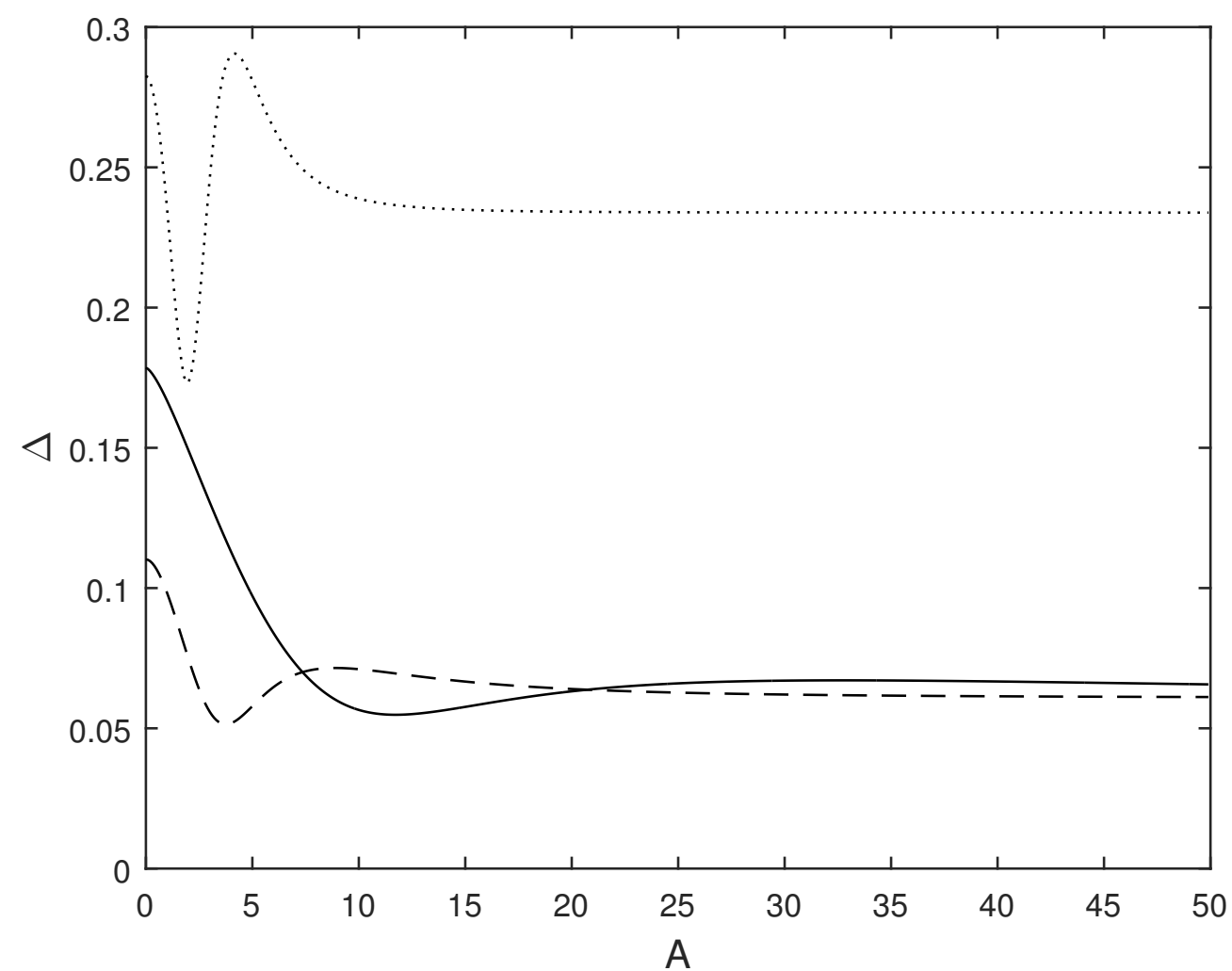

Figura 9 - Erros relativo, $\Delta$ (Algoritmo 2), versus amplitude, $A$, para $v=10$, e $\alpha=1 / 2$ (linha contínua), $\alpha=3 / 2$ (linha tracejada) e $\alpha=3$ (linha pontilhada). 


\subsection{Vibração livre de osciladores com amortecimento linear}

Nesta seção é analisada a vibração livre que ocorre em osciladores não-lineares com qualquer ordem de não-linearidade (inteira e não-inteira) na presença de significante amortecimento viscoso, no qual possui uma linearidade proporcional a velocidade.

Os osciladores mecânicos não-lineares não-conservativos tratados na presente seção têm a seguinte equação de movimento geral:

$$
\ddot{x}+\kappa \dot{x}+c_{1}^{2} x+c_{\alpha}^{2} x|x|^{\alpha-1}=0,
$$

com as seguintes condições iniciais:

$$
x(0)=A, \quad \dot{x}(0)=v,
$$

onde $\kappa$ é a constante de amortecimento linear, $c_{1}^{2}$ é a constante da força conservativa linear, $c_{\alpha}^{2}$ é a constante da força conservativa não-linear e $\alpha$ é a ordem de não-linearidade inteira ou não-inteira.

A equação (3.128) será resolvida usando série de Taylor que, recentemente, por causa da solução iterativa, diversos autores referenciam a solução descrita na presente seção por um método chamado de Método da Transformada (ou Transformação) Diferencial (DTM - "Differential Transformation Method"), como distinto ou como uma generalização do método de Taylor tradicional. Esse método DTM de Pukhov (1978), Pukhov (1981), Pukhov (1982) e Zhou (1986) (trabalho escrito em Chinês) é frequentemente utilizado para resolver equações diferenciais. O artigo de Bervillier (2012) apresenta um extensa revisão bibliográfica do método DTM comparando com o método da série de Taylor. Bervillier (2012) conclui que a maior contribuição do método DTM está em uma fácil generalização do método de Taylor (quer para soluções numéricas ou analíticas de equações diferenciais) para resolver problemas com derivadas não-usuais, como fracionais, fuzzy, ou derivadas- $q$ (KAC; CHEUNG, 2002). Dentro dessa linha de aplicação do DTM, na presente seção, deriva-se um procedimento para a solução de equações com não-linearidades racionais do tipo da Eq. (3.128) baseado na expansão de Taylor.

Hairer, Nørsett e Wanner (1993) atribuem a Newton a ideia de utilizar séries infinitas para resolver equações diferenciais. Newton (1671) considera a solução de equações diferenciais por meio de expansão em séries. Um dos primeiros exemplos de equação de primeira ordem tratada por Newton (1671) é expressa por $y^{\prime}=1-3 x+y+x^{2}+x y$. Considerando a série de Taylor, a solução genérica de uma equação geral, $f\left(t, x, x^{\prime}, \ldots x^{(n)}\right)=$ 0 , com condições iniciais, $x^{(k)}\left(t_{i}\right)=d_{k i}, k=0, \ldots, n-1$, é expressa localmente como uma expansão truncada de Taylor em torno do instante $t_{i}$ (instante inicial $t_{0}$ ou instante intermediário de um procedimento de múltiplos passos $t_{i}=t_{0}+i h$ ) (ver (BERVILLIER, 2012)): 


$$
\begin{aligned}
x(t) & =\sum_{k=0}^{N}\left(t-t_{i}\right)^{k} X_{k}+O\left[\left(t-t_{i}\right)^{N+1}\right], \\
x\left(t_{i}\right) & =d_{k i}, \\
X_{k} & =\left.\frac{1}{k !} \frac{d^{k} x}{d t^{k}}\right|_{t=t_{i}}, k=0,1,2, \ldots, N .
\end{aligned}
$$

Os coeficientes $X_{k}$ são determinados de modo que a equação diferencial ordinária seja satisfeita até a ordem $O\left[\left(t-t_{i}\right)^{N}\right]$ sujeita às condições iniciais ou por uma equação de interação, por exemplo, considerando uma equação de primeira ordem $y^{\prime}=f(x, t), y\left(t_{0}\right)=$ $d_{0}$ e supondo que $f(x, t)$ seja composta de uma sequência de operações algébricas e funções elementares, de forma que para cada termo de $f$ determina-se uma fórmula do $k$-ésimo coeficiente de Taylor como descrito a seguir.

a) $z(t)=x(t)$ :

$$
Z_{k}=X_{k}=\left.\frac{1}{k !} \frac{d^{k} x}{d t^{k}}\right|_{t=t_{i}}
$$

b) $z(t)=\lambda x(t)$ :

$$
Z_{k}=\lambda X_{k}
$$

c) $z(t)=x(t)+y(t)$ :

$$
Z_{k}=X_{k}+Y_{k}=\left.\frac{1}{k !} \frac{d^{k} x}{d t^{k}}\right|_{t=t_{i}}+\left.\frac{1}{k !} \frac{d^{k} y}{d t^{k}}\right|_{t=t_{i}}
$$

d) $z(t)=\frac{d^{n} x}{d t^{n}}$ :

$$
Z_{k}=\left.\frac{1}{k !} \frac{d^{k}\left(d^{n} x\right)}{d t^{k}\left(d t^{n}\right)}\right|_{t=t_{i}}=\left.\frac{(k+n) !}{k !(k+n) !} \frac{d^{k+n} x}{d t^{k+n}}\right|_{t=t_{i}}=\frac{(k+n) !}{k !} Z_{k+n}=(k+n) \ldots(k+1) Z_{k+n}
$$

e) $z(t)=x(t) y(t)$ :

$$
Z_{k}=\left.\frac{1}{k !} \frac{d^{k}(x(t) y(t))}{d t^{k}}\right|_{t=t_{i}}
$$

e usando a regra geral de Leibntz $(u v)^{(n)}=\sum_{k=0}^{n}\left(\begin{array}{l}n \\ k\end{array}\right) u^{(k)} v^{(n-k)}$ onde $\left(\begin{array}{l}n \\ k\end{array}\right)=\frac{n !}{k !(n-k) !}$ é o coeficiente binomial, tem-se que: 


$$
\begin{aligned}
Z_{k} & =\left.\frac{1}{k !} \frac{d^{k}(x(t) y(t))}{d t^{k}}\right|_{t=t_{i}}=\left.\frac{1}{k !} \sum_{i=0}^{k}\left(\begin{array}{c}
k \\
i
\end{array}\right) x^{(i)} y^{(k-i)}\right|_{t=t_{i}}=\left.\frac{1}{k !} \sum_{i=0}^{k} \frac{k !}{i !(k-i) !} x^{(i)} y^{(k-i)}\right|_{t=t_{i}} \\
& =\left.\sum_{i=0}^{k} \frac{x^{(i)}}{i !} \frac{y^{(k-i)}}{(k-i) !}\right|_{t=t_{i}}=\sum_{i=0}^{k} X_{i} Y_{k-i}, k \geq 0 .
\end{aligned}
$$

Esse resultado pode também ser derivado usando o produto de Cauchy.

f) $z(t)=x(t)^{\alpha}$ :

Nesse caso deve-se considerar a potenciação com uma arbitrária potência $\alpha$ de uma série de potências. Euler (1748, parágrafo 76) descobriu um método simples e eficiente para a obtenção de potenciação de séries de potência (ver também, Knuth (1997), Henrici (1956) e Hairer, Nørsett e Wanner (1993)).

$$
Z_{k}=\left.\frac{1}{k !} \frac{d^{k}\left(x(t)^{\alpha}\right)}{d t^{k}}\right|_{t=t_{i}}
$$

Diferenciando $z(t)=x(t)^{\alpha}$ tem-se que $\dot{z} x=\alpha z \dot{x}$ e usando as Eqs. (3.134) e (3.136) obtém-se a seguinte relação:

$$
\sum_{i=0}^{k} i Z_{i} X_{k-i}=\sum_{i=0}^{k} \alpha(k-i) Z_{i} X_{k-i}
$$

e de acordo com a Eq. (3.138) deriva-se a expressão recursiva:

$$
\begin{aligned}
& Z_{0}=X_{0}^{\alpha} \\
& Z_{k}=\frac{1}{k X_{0}}\left(\sum_{i=0}^{k-1}(\alpha k-(\alpha+1) i) Z_{i} X_{k-i}\right), k \geq 1
\end{aligned}
$$

A Eq. (3.139) pode ser utilizada para potências arbitrárias, como: $\alpha=2, \alpha=1 / 2$, $\alpha=-10, \alpha=\pi$, entre outras. Outras fórmulas para os coeficientes de Taylor são derivadas na literatura para termos de $f$ mais complicados como exponenciais, logaritmos, funções trigonométricas, entre outras (ver, por exemplo, o livro de Hairer, Nørsett e Wanner (1993)).

A equação de movimento do oscilador não-conservativo, Eq. (3.128), pode ser reescrita como:

$$
\ddot{x}+\kappa \dot{x}+c_{1}^{2} x+c_{\alpha}^{2} \operatorname{sign}(x)|x|^{\alpha}=0,
$$

onde $\operatorname{sign}(x)$ é a função sinal de $x$ com valor de +1 para $x>0$ e de -1 para $x<0$. Utilizando a expansão truncada de Taylor em torno do instante $t_{i}$ e as relações, Eqs.(3.131), (3.132), (3.133), (3.134), (3.136, (3.139), na equação de movimento, Eq. (3.140), e nas condições iniciais, Eq. (3.129), obtém-se: 


$$
\begin{aligned}
X(k+2) & =\frac{\left(-\kappa(k+1) X(k+1)-c_{1}^{2} X(k)-c_{\alpha}^{2} \operatorname{sign}(X(k))|R(k)|\right)}{(k+1)(k+2)}, k \geq 0, \\
X(0) & =A, X(1)=v, \\
R(0) & =X(0)^{\alpha}, \\
R(k) & =\frac{1}{k X(0)}\left(\sum_{i=0}^{k-1}(\alpha k-(\alpha+1) i) R(i) X(k-i)\right), k \geq 1 .
\end{aligned}
$$

A solução da Eq.(3.128) é obtida por meio da série, Eq.(3.130), e dos coeficientes dados por (3.141). Venkatarangan e Rajalakshmi (1995) propuseram um tratamento da solução obtida por meio da decomposição de Adomian (ADOMIAN, 1986) para sistemas oscilatórios não-lineares. Jiao et al. (2002) menciona que a soluções obtidas pela decomposição de Adomian é uma solução em série de Taylor (ou série de Maclaurin), isto é, na prática uma série truncada. O método da decomposição de Adomian é aplicado diretamente para diversos tipos de equações funcionais, lineares ou não-lineares. O tratamento proposto por Venkatarangan e Rajalakshmi (1995) foi motivado pois a solução obtida na forma de série não exibia periodicidade que é uma característica de sistemas oscilatórios. Para contornar esse problema, os autores aplicaram a transformada de Laplace na série obtida pelo método de decomposição de Adomian, na sequência converteram a série transformada em uma função formada pelos aproximantes de Padé (ver (PADé, 1892), escrito em Francês), e então inverteram os aproximantes obtendo uma solução periódica. Essa técnica de tratamento adicional da série obtida é discutida e aplicada por diversos autores, como Jiao et al. (2002), Momani (2004), Momani e Ertürk (2008), El-Shahed (2008), entre outros. Jiao et al. (2002) descreve que essa técnica de pós-tratamento da série obtida pelos aproximantes de Padé melhora a região e taxa de convergência em relação a série original, obtendo melhores soluções analíticas aproximadas que as séries truncadas de Adomian. Para sistemas oscilatórios o pós-tratamento da série com transformada de Laplace e aproximantes de Padé melhora a acuracidade das soluções analíticas para esses sistemas.

A transformada de Laplace de uma função $f(t)$ real ou complexa e um parâmetro $s$ real ou complexo é definida por (ver p. ex. (ROY; OLVER; WONG, 2010)):

$$
\mathcal{L}(f(t) ; s)=\int_{0}^{\infty} e^{-s t} f(t) d t
$$

e a inversa da transformada de Laplace é definida se $f(t)$ é contínua e $f^{\prime}(t)$ é contínua por partes em $[0, \infty)$ (ver p. ex. (ROY; OLVER; WONG, 2010)):

$$
f(t)=\frac{1}{2 \pi i} \lim _{T \rightarrow \infty}=\int_{\sigma-i T}^{\sigma-i T} e^{t s} \mathcal{L}(f(t) ; s) d s,
$$

onde $\sigma$ é um número real e $i=\sqrt{-1}$. 
Padé (1892) publicou um trabalho que trata das representações aproximadas de uma função por funções racionais. Essas funções são conhecidas por aproximantes de Padé. Segundo Aguilera-Navarro et al. (1999) o método de Padé permite a partir de uma série de potências obter mais informações do que a própria série pode fornecer diretamente, isto é, os aproximantes de Padé convergem mais rapidamente o que a série de Taylor e se estendem a regiões maiores da definida pelo raio de convergência da série de Taylor. De acordo com Baker (1975) o aproximante de Padé $[L / M]$ para uma série de potências $A(x)=\sum_{j=0}^{N} a_{j} x^{j}$ é definido como a razão de dois polinômios construídos a partir dos coeficientes da série $A(x)$ que pode uma série de Taylor originária da expansão de uma função. O aproximante de Padé $[L / M]$ é representado por (BAKER, 1975):

$$
[L / M]=P_{L}(x) / Q_{M}(x), L, M \geq 0,
$$

onde $P_{L}(x)$ e $Q_{M}(x)$ são polinômios de graus menores do que $L$ e $M$, respectivamente, dados por:

$$
P_{L}(x)=p_{0}+p_{1} x+p_{2} x^{2}+\ldots+p_{L} x^{L}
$$

e

$$
Q_{M}(x)=q_{0}+q_{1} x+q_{2} x^{2}+\ldots+q_{M} x^{M}
$$

Como a multiplicação do numerador e denominador por uma constante mantém a Eq. (3.144) inalterada, pode-se normalizar essa expressão fazendo $q_{0}=1$. Os coeficientes $p_{i} \mathrm{e}$ $q_{j}$ são determinados a partir de $A(x)$ através da equação

$$
f(x)-\frac{P_{L}(x)}{Q_{M}(x)}=\mathcal{O}\left(x^{L+M+1}\right)
$$

que requer que a função $A(x)$ e o aproximante de Padé $[L / M]$ diferem apenas nos termos de ordem $\mathcal{O}\left(x^{L+M+1}\right)$. A partir da Eq. (3.147) pode-se escrever

$$
P_{L}(x)=f(x) Q_{M}(x)+\mathcal{O}\left(x^{L+M+1}\right)
$$

onde a Eq. (3.148) representa um sistema de $L+M+1$ equações algébricas para calcular os coeficientes dos polinômios $P_{L}(x)$ e $Q_{M}(x)$. A função $A(x)$ deve ser truncada com a quantidade de termos suficientes para fornecer informações para os aproximantes de Padé, isto é, $L+M \leq N$. Quando $q_{0}=1$, o aproximante de Padé é único para um determinado $L \mathrm{e}$ $M$, ou seja, o aproximante de Padé para a função truncada $A(x)$ é unicamente determinado pelo sistema de equações dado por (3.148) para um dado $L$ e $M$ quando $N \geq L+M$ (ver (JIAO et al., 2002)). Os valores $L$ e $M$ são inteiros não-negativos e se $L=M$ o aproximante de Padé correspondente é denominado diagonal (AGUILERA-NAVARRO et al., 1999). Caso $M=0$ e $q_{0}=1$ a expansão de $A(x)$ coincide com o polinômio $P_{L}(x)$. 


\subsubsection{Exemplo de oscilador conservativo}

A metodologia descrita acima é primeiramente exemplificada em um oscilador mecânico conservativo, $\kappa=0$, com não-linearidade fracionária, $\alpha=5 / 2$, governado pela seguinte equação de movimento geral:

$$
\ddot{x}+x|x|^{\alpha-1}=0
$$

com as seguintes condições iniciais:

$$
x(0)=1, \quad \dot{x}(0)=0 .
$$

Utilizando a transformada da Eq. (3.141) na equação de movimento, Eq. (3.149), e nas condições iniciais, Eq. (3.150), obtém-se:

$$
\begin{aligned}
X(k+2) & =-\frac{\operatorname{sign}(X(k))|R(k)|}{(k+1)(k+2)}, k \geq 0, \\
X(0) & =1, X(1)=0, \\
R(0) & =X(0)^{5 / 2}, \\
R(k) & =\frac{1}{2 k X(0)}\left(\sum_{i=0}^{k-1}(5 k-7 i) R(i) X(k-i)\right), k \geq 1 .
\end{aligned}
$$

Utilizando a Eq. (3.151) e a expansão truncada de Taylor em torno do instante inicial $t_{i}=t_{0}=0$ obtém-se para $N=16$ :

$$
\begin{array}{r}
x(t)=1.0-0.5 t^{2}+0.104167 t^{4}-0.0243056 t^{6}+0.00527034 t^{8} \\
-0.00112296 t^{10}+0.000237108 t^{12}-0.0000495683 t^{14}+0.0000103054 t^{16}
\end{array}
$$

A solução obtida, Eq. (3.152), não tem característica periódica. Assim, aplica-se o pós-tratamento da solução proposto por Venkatarangan e Rajalakshmi (1995), isto é, utiliza-se a transformada de Laplace, Eq. (3.142), na série obtida, na sequência converte a série transformada em uma função formada pelos aproximantes de Padé, Eq. (3.144), e, finalmente, utiliza-se a transformada inversa de Laplace, Eq.(3.143). Esse procedimento é detalhado a seguir. A transformada de Laplace da Eq.(3.152) resulta em:

$$
\begin{array}{r}
\mathcal{L}(x(t) ; s)=\frac{2.15617 \times 10^{8}}{s^{17}}-\frac{4.32128 \times 10^{6}}{s^{15}}+\frac{113575}{s^{13}} \\
-\frac{4075}{s^{11}}+\frac{212.5}{s^{9}}-\frac{17.5}{s^{7}}+\frac{2.5}{s^{5}}-\frac{1}{s^{3}}+\frac{1}{s}
\end{array}
$$

Para fazer a transformação de Padé, a Eq. (3.153) é convertida na forma de um polinômio através da seguinte troca de variável $s=1 / z$ : 


$$
\begin{aligned}
& x^{*}(z)=z-z^{3}+2.5 z^{5}-17.5 z^{7}+212.5 z^{9}-4075 z^{11} \\
& \quad+113575 z^{13}-4.32128 \times 10^{6} z^{15}+2.15617 \times 10^{8} z^{17}
\end{aligned}
$$

A Eq. (3.154) é transformada na razão de dois polinômios através da construção do aproximante de Padé (Eq. 3.144). Os valores de $L$ e $M$ devem ser escolhidos de forma que $L+M \leq N$. Considerando $L=M=8$, obtém-se:

$$
\left[\frac{L}{M}\right]=\left[\frac{8}{8}\right]=\frac{1.0 z+96.3144 z^{3}+2182.82 z^{5}+10648.6 z^{7}}{1.0+97.3144 z^{2}+2277.63 z^{4}+12700.5 z^{6}+8496.88 z^{8}}
$$

Fazendo novamente a troca de variável, $z=1 / s$, na Eq. (3.155) e usando a inversa da transformada de Laplace (Eq. 3.143) resulta em:

$$
\begin{aligned}
& x(t)=0.964251 \cos (0.878948 t)+0.353962 \times 10^{-1} \cos (2.63908 t) \\
& +0.352719 \times 10^{-3} \cos (4.91206 t)+5.08118 \times 10^{-7} \cos (8.09005 t)
\end{aligned}
$$

A figura 10 mostra a comparação entre a solução numérica da Eq. (3.149) com condições iniciais (3.150) obtida pelo método de Runge-Kutta (ver linha contínua) e a solução aproximada, Eq. (3.156), linha tracejada. As soluções são plotadas durante o intervalo de tempo $0 \leq t \leq 60$. O erro relativo médio entre as soluções numérica e aproximada no intervalo de tempo $0 \leq t \leq 60$ com incremento temporal $\Delta t=0.001$ é $6.3139 \%$ e o erro médio absoluto é 0.0024722 . A figura 10b ilustra uma amplificação da solução no intervalo $7 \leq t \leq 7.3$ em que se pode notar a diferença entre as soluções. Essa diferença é perceptível somente na quarta casa decimal dos valores numéricos das soluções.

Utilizando a Eq. (3.45) a solução aproximada obtida pela abordagem Hamiltoniana aplicada para uma função trigonométrica de primeira ordem é expressa por:

$$
x(t)=\cos (0.891562 t) .
$$

A solução aproximada obtida pela Eq. (3.156) apresenta termos de alta ordem sendo os dois primeiros predominantes na solução. O período de vibração aproximado obtido para a Eq.(3.157) é de $T=7.04739$ (Eq. 3.46) que difere do período exato (ver Eq. 3.48) $T_{e x}=7.14736$ em $1.3987 \%$.

A figura 11 mostra a comparação entre a solução numérica da Eq. (3.149) com condições iniciais (3.150) (linha contínua), a solução aproximada, Eq. (3.156), (linha tracejada) e a solução aproximada, Eq.(3.157), (linha pontilhada) durante o intervalo de tempo $0 \leq t \leq 10$. Observa-se na figura 11 b (ver defasagem das soluções) que a solução 


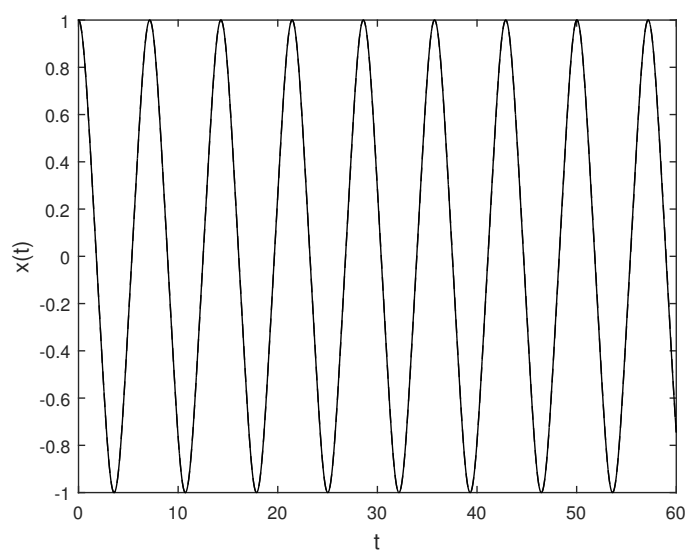

(a)

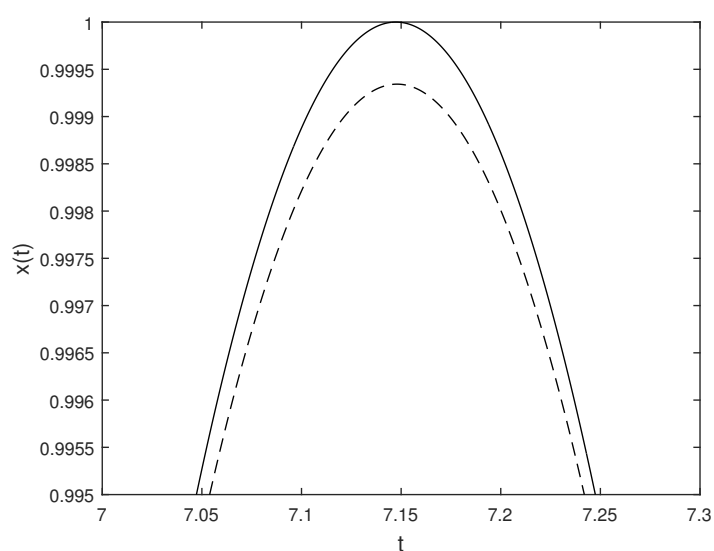

(b)

Figura 10 - Solução numérica, $x(t)$, da Eq. (3.149) (linha contínua) e solução aproximada, $x(t)$, (Eq. 3.156) (linha tracejada): (a) $0 \leq t \leq 60$; (b) $7 \leq t \leq 7.3$.

aproximada pela série de Taylor é mais precisa que a solução obtida pela abordagem Hamiltoniana. Essa diferença se deve ao fato que foi utilizada uma função trigonométrica simples na solução da abordagem Hamiltoniana (com frequência de oscilação 0.891562) e, por causa da não-linearidade, composições de outros termos harmônicos da frequência são relevantes. Observa-se, na solução da Eq. (3.156), que o primeiro termo oscila com uma frequência $\omega_{1}=0.878948$ enquanto o segundo com $\omega_{2} \approx 3 \omega_{1} \approx 2.63908$.

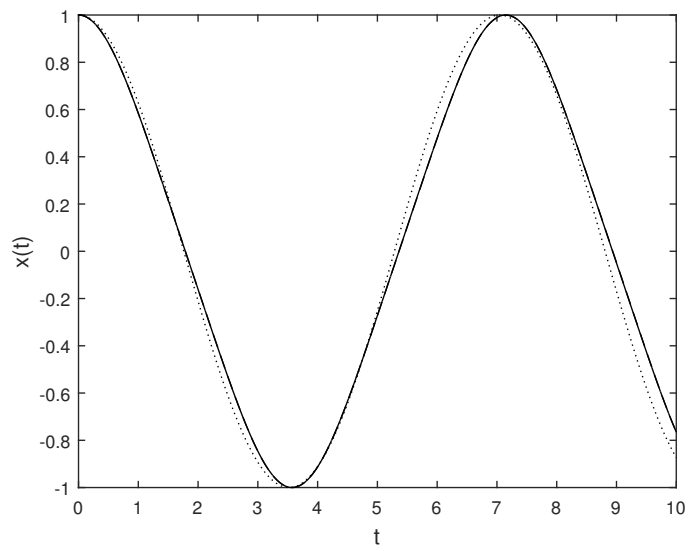

(a)

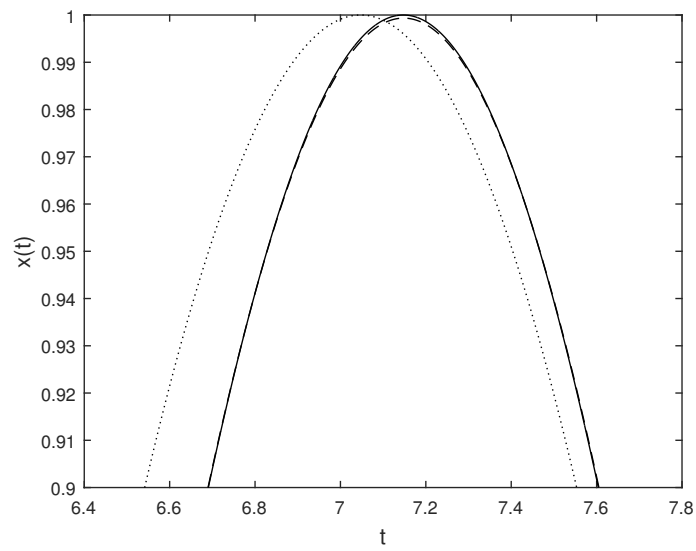

(b)

Figura 11 - Solução numérica, $x(t)$, da Eq. (3.149) (linha contínua), solução aproximada, $x(t)$, (Eq. 3.156) (linha tracejada) e solução aproximada, $x(t)$, Eq.(3.157), (linha pontilhada): (a) $0 \leq t \leq 10$; (b) $6.4 \leq t \leq 7.8$.

\subsubsection{Exemplos de osciladores não-conservativos}

Nesta seção são apresentados alguns exemplos de vibração livre de osciladores não-conservativos com amortecimento linear. A metodologia baseada na expansão em série de Taylor é primeiramente exemplificada em um oscilador mecânico não-conservativo, 
$\kappa=1$, com não-linearidade cúbica $\alpha=3$ (oscilador não-linear de Duffing) governado pela seguinte equação de movimento geral $\left(c_{1}^{2}=20, c_{\alpha}^{2}=2\right.$, ver (NOURAZAR; MIRZABEIGY, 2013)):

$$
\ddot{x}+\kappa \dot{x}+c_{1}^{2} x+c_{\alpha}^{2} x|x|^{\alpha-1}=\ddot{x}+\dot{x}+20 x+2 x^{3}=0,
$$

com as seguintes condições iniciais:

$$
x(0)=-0.2, \quad \dot{x}(0)=2 .
$$

Utilizando a transformada da Eq. (3.141) na equação de movimento, Eq. (3.158), e nas condições iniciais, Eq. (3.159), obtém-se:

$$
\begin{aligned}
X(k+2) & =-\frac{((k+1) X(k+1)+20 X(k)+2 \operatorname{sign}(X(k))|R(k)|)}{(k+1)(k+2)}, k \geq 0, \\
X(0) & =-0.2, X(1)=2, \\
R(0) & =X(0)^{3} \\
R(k) & =\frac{1}{k X(0)}\left(\sum_{i=0}^{k-1}(3 k-4 i) R(i) X(k-i)\right), k \geq 1 .
\end{aligned}
$$

Utilizando a Eq. (3.160) e a expansão truncada de Taylor em torno do instante inicial $t_{i}=t_{0}=0$ obtém-se para $N=4$ :

$$
x(t)=-0.2+2.0 t+1.008 t^{2}-7.082667 t^{3}-0.2891733 t^{4}
$$

A transformada de Laplace da Eq.(3.161) resulta em:

$$
\mathcal{L}(x(t) ; s)=-\frac{0.2}{s}+\frac{2}{s^{2}}+\frac{2.016}{s^{3}}-\frac{42.496}{s^{4}}-\frac{6.94016}{s^{5}}
$$

Fazendo a seguinte troca de variável $s=1 / z$ na Eq. (3.162) obtém-se:

$$
x^{*}(z)=-0.2 z+2 z^{2}+2.016 z^{3}-42.496 z^{4}-6.94016 z^{5}
$$

A partir da Eq. (3.163) constrói-se o aproximante de Padé (Eq. 3.144) para valores $L=M=2, L+M \leq N=4$ :

$$
\left[\frac{L}{M}\right]=\left[\frac{2}{2}\right]=\frac{-0.2 z+1.797093 z^{2}}{1.0+1.014535 z+20.2253 z^{2}}
$$

Utilizando a troca de variável $z=1 / s$ na Eq. (3.164) e a inversa da transformada de Laplace (Eq. 3.143) resulta em: 


$$
\begin{array}{r}
x(t)=-0.2 \exp (-0.507267 t) \cos (4.46856 t) \\
+0.424868 \exp (-0.507267 t) \sin (4.46856 t)
\end{array}
$$

A figura 12 mostra a comparação entre a solução numérica da Eq. (3.158) com condições iniciais (3.159) obtida pelo método de Runge-Kutta (ver linha contínua) e a solução aproximada, Eq. (3.165), linha tracejada. As soluções são plotadas durante o intervalo de tempo $0 \leq t \leq 10$. O erro relativo médio entre as soluções numérica e aproximada no intervalo de tempo $0 \leq t \leq 10$ com incremento temporal $\Delta t=0.001$ é $82.937 \%$ e o erro médio absoluto é 0.0017742 . A figura 12b ilustra uma amplificação da solução no intervalo $1 \leq t \leq 5$ em que se pode notar a diferença entre as soluções.

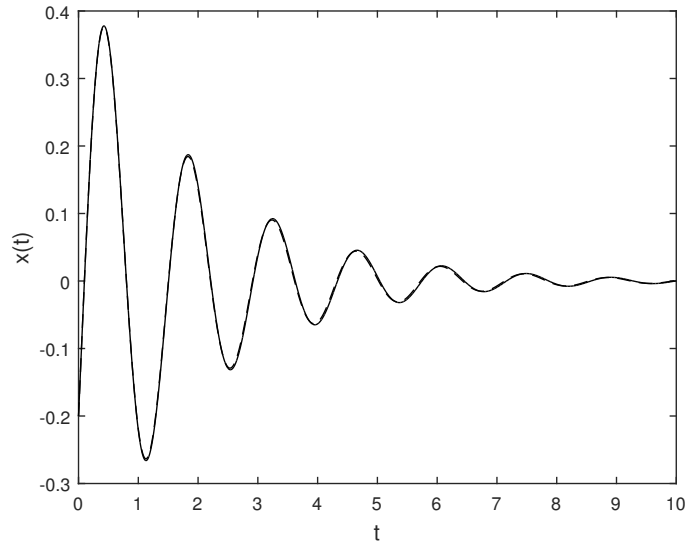

(a)

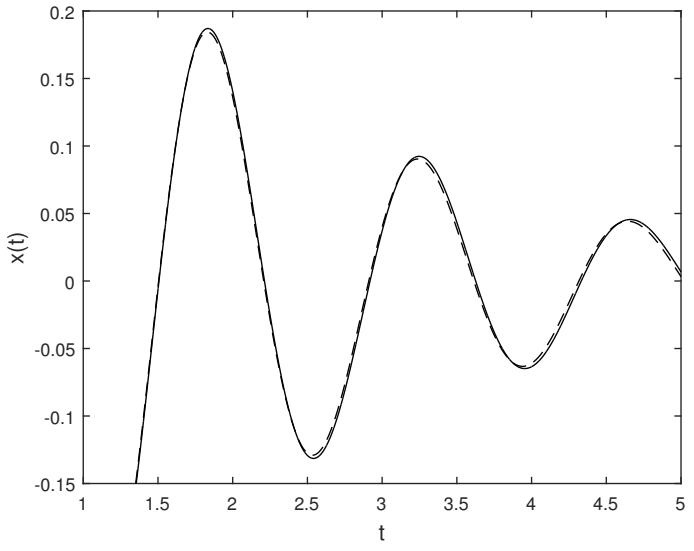

(b)

Figura 12 - Solução numérica, $x(t)$, da Eq. (3.149) (linha contínua) e solução aproximada, $x(t)$, (Eq. 3.156) (linha tracejada): (a) $0 \leq t \leq 10$; (b) $1 \leq t \leq 5$.

A seguir utiliza-se a expansão em série de Taylor em um oscilador mecânico nãoconservativo, $\kappa=2$, com não-linearidade não-inteira $\alpha=3 / 2$ segundo a seguinte equação de movimento geral $\left(c_{1}^{2}=10, c_{\alpha}^{2}=1\right)$ :

$$
\ddot{x}+\kappa \dot{x}+c_{1}^{2} x+c_{\alpha}^{2} x|x|^{\alpha-1}=\ddot{x}+2 \dot{x}+10 x+x \sqrt{|x|}=0,
$$

com as seguintes condições iniciais:

$$
x(0)=0.1, \quad \dot{x}(0)=0 .
$$

Utilizando a transformada da Eq. (3.141) na equação de movimento, Eq. (3.166), e nas condições iniciais, Eq. (3.167), obtém-se: 


$$
\begin{aligned}
X(k+2) & =-\frac{(2(k+1) X(k+1)+10 X(k)+\operatorname{sign}(X(k))|R(k)|)}{(k+1)(k+2)}, k \geq 0, \\
X(0) & =0.1, X(1)=0, \\
R(0) & =X(0)^{3 / 2} \\
R(k) & =\frac{1}{2 k X(0)}\left(\sum_{i=0}^{k-1}(3 k-5 i) R(i) X(k-i)\right), k \geq 1 .
\end{aligned}
$$

Utilizando a Eq. (3.168) e a expansão truncada de Taylor em torno do instante inicial $t_{i}=t_{0}=0$ obtém-se para $N=4$ :

$$
x(t)=0.1-0.5158114 t^{2}+0.3438743 t^{3}+0.2782949 t^{4}
$$

A transformada de Laplace da Eq.(3.169) resulta em:

$$
\mathcal{L}(x(t) ; s)=\frac{0.1}{s}-\frac{1.031623}{s^{3}}+\frac{2.063246}{s^{4}}+\frac{6.679078}{s^{5}}
$$

Fazendo a seguinte troca de variável $s=1 / z$ na Eq. (3.170) obtém-se:

$$
x^{*}(z)=0.1 z-1.031623 z^{3}+2.063246 z^{4}+6.679078 z^{5}
$$

A partir da Eq. (3.171) constrói-se o aproximante de Padé (Eq. 3.144) para valores $L=M=2, L+M \leq N=4$ :

$$
\left[\frac{L}{M}\right]=\left[\frac{2}{2}\right]=\frac{0.1 z+0.2 z^{2}}{1.0+2.0 z+10.31623 z^{2}}
$$

Utilizando a troca de variável $z=1 / s$ na Eq. (3.172) e a inversa da transformada de Laplace (Eq. 3.143) resulta em:

$$
x(t)=0.1 \exp (-t) \cos (3.05225 t)+0.0327627 \exp (-t) \sin (3.05225 t)
$$

As condições iniciais, $x(0)=0.1$ e $\dot{x}(0)=0$, são válidas na equação (3.173). A figura 13 mostra a comparação entre a solução numérica da Eq. (3.166) com condições iniciais (3.167) obtida pelo método de Runge-Kutta (ver linha contínua) e a solução aproximada, Eq. (3.173), linha tracejada. As soluções são plotadas durante o intervalo de tempo $0 \leq t \leq 10$. O erro relativo médio entre as soluções numérica e aproximada no intervalo de tempo $0 \leq t \leq 10$ com incremento temporal $\Delta t=0.001$ é $97.5 \%$ e o erro médio absoluto é 0.0001229. A figura 13b ilustra um zoom da solução no intervalo $0.5 \leq t \leq 5.5$ em que se pode notar a diferença entre as soluções. As duas soluções seguem bem próximas para o regime estacionário que ocorre no instante aproximado de $t=5.5$. 


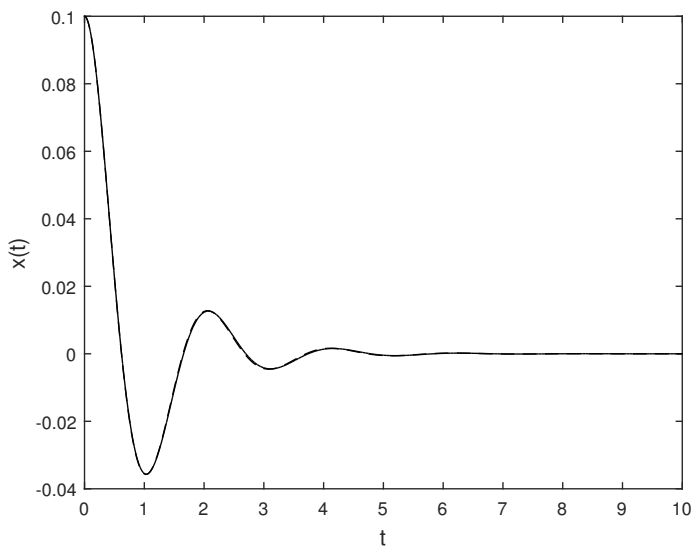

(a)

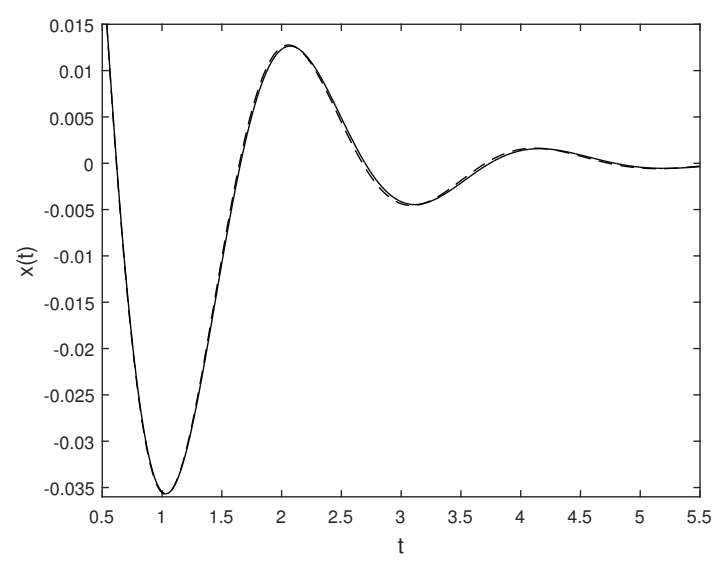

(b)

Figura 13 - Solução numérica, $x(t)$, da Eq. (3.149) (linha contínua) e solução aproximada, $x(t)$, (Eq. 3.156) (linha tracejada): (a) $0 \leq t \leq 10$; (b) $0.5 \leq t \leq 5.5$. 



\section{Modelos de Contato}

Na abordagem do Método dos Elementos Discretos (DEM), o movimento de uma partícula material $i$ com massa $m^{(i)}$ é descrito pela segunda lei de Newton como:

$$
m^{(i)} \frac{d^{2} \mathbf{X}^{(i)}}{d t^{2}}=\mathbf{F}^{(i)}(t)=m^{(i)} \mathbf{g}+\mathbf{F}_{c}^{(i)}(t)
$$

onde $\mathbf{X}^{(i)}$ é a posição do centro de massa da partícula e $\mathbf{F}^{(i)}$ é a soma de todas forças externas agindo na partícula $i$. As forças externas são divididas em forças de campo (ex.: força gravitacional, $m^{(i)} \mathbf{g}$ ) e forças de contato (ex.: força de contato agindo na partícula $i$ devido à colisão com outras partículas, $\mathbf{F}_{c}^{(i)}$ ). $\mathrm{O}$ momento das forças externas atuando na partícula $i$ é calculado por:

$$
\mathbf{T}^{(i)}(t)=I^{(i)} \frac{d \vec{\omega}^{(i)}(t)}{d t}
$$

onde $I^{(i)}$ é o momento de inércia de massa da partícula, que para a forma esférica é expresso por: $I^{(i)}=\frac{2}{5} m^{(i)} r^{(i)^{2}}$, onde $r^{(i)}$ é o raio da partícula.

Para um contato "não instantâneo", as forças de contato no método DEM são calculadas de acordo com o modelo de "esfera-suave" proposto por (CUNDALL; STRACK, 1979). Este modelo é baseado em um sistema dinâmico massa-mola amortecedor que permite análise da interação entre partículas e entre partículas e uma parede rígida. No modelo de "esfera-suave", as forças de contato são calculadas a partir da sobreposição não-física ("deformação", $\delta$ ) e da velocidade relativa entre as partículas.

A Figura 14 (CUNDALL; STRACK, 1979) ilustra a colisão entre duas partículas ( $i$ e $j$, de diâmetros $D^{(i)}$ e $D^{(j)}$ e posições dos centros de massas $\mathbf{X}^{(i)}$ e $\mathbf{X}^{(j)}$ ) no modelo esfera-suave. A sobreposição das partículas $\delta_{n}$ na direção normal é expressa por:

$$
\delta_{n}=0,5\left(D^{(i)}+D^{(j)}\right)-\left|\mathbf{X}^{(i)}-\mathbf{X}^{(j)}\right|
$$

onde $\mathbf{X}^{(i)}$ e $\mathbf{X}^{(j)}$ são as posições dos centros de massas das partículas $i$ e $j$, respectivamente. Para as partículas com velocidades lineares e angulares iguais a $\mathbf{V}^{(i)}$ e $\mathbf{w}^{(i)}$, e $\mathbf{V}^{(j)}$ e $\mathbf{w}^{(j)}$, respectivamente, a velocidade relativa entre as partículas no ponto de contato é:

$$
\mathbf{V}_{i j}=\mathbf{V}^{(j)}-\mathbf{V}^{(i)}+\vec{\omega}^{(j)} \times \mathbf{R}^{(j)}-\vec{\omega}^{(i)} \times \mathbf{R}^{(i)}
$$

onde $\mathbf{R}^{(i)}$ e $\mathbf{R}^{(j)}$ são vetores com origem nos centros de massas das partículas $i$ e $j$, respectivamente, até o ponto de contato (Figura 14).

As componentes normal $\mathbf{V}_{n, i j}$ e tangencial $\mathbf{V}_{t, i j}$ da velocidade relativa no ponto de contato são expressas por:

$$
\mathbf{V}_{n, i j}=\left(\mathbf{V}_{i j} \cdot \mathbf{n}_{i j}\right) \mathbf{n}_{i j}
$$




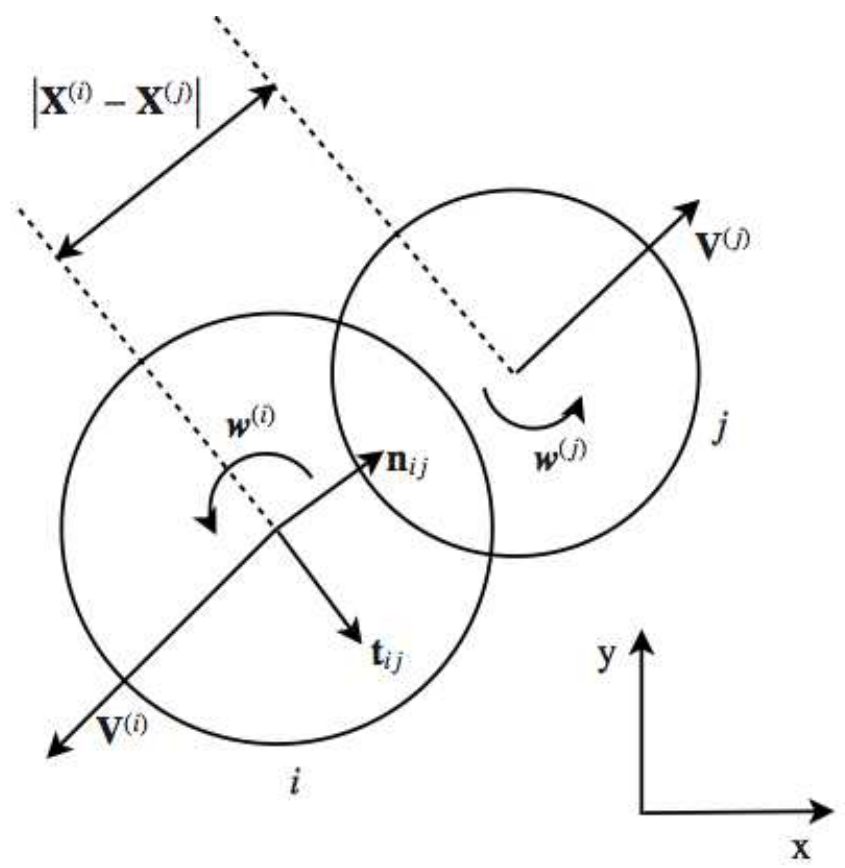

Figura 14 - Esquema de contato entre dois corpos no modelo "esfera-suave" (CUNDALL; STRACK, 1979)

$$
\mathbf{V}_{t, i j}=\mathbf{V}_{i j}-\mathbf{V}_{n, i j}
$$

$\mathbf{n}_{i j}=\left(\mathbf{X}^{(j)}-\mathbf{X}^{(i)}\right) /\left|\mathbf{X}^{(j)}-\mathbf{X}^{(i)}\right|$ é o vetor unitário normal ao longo da linha de contato direcionado da partícula $i$ para a partícula $j$ e $\mathbf{t}_{i j}=\mathbf{V}_{t, i j} /\left|\mathbf{V}_{t, i j}\right|$ é o vetor unitário na direção tangencial. No modelo colisional de esfera-suave, a sobreposição $(\delta)$ entre duas partículas é representada por um sistema massa-mola-amortecedor nas direções normal e tangencial. A mola representa as forças conservativas e o amortecedor as forças dissipativas (ver Figura 15).

A força de contato $\left(\mathbf{F}_{c}^{(i)}\right)$ agindo em uma partícula $i$, no instante $t$, é calculada como a soma das forças de contato de todas partículas $j$ que estão em contato com a partícula $i$ :

$$
\mathbf{F}_{c}^{(i)}(t)=\sum_{j=1}^{N}\left(\mathbf{F}_{n, i j}(t)+\mathbf{F}_{t, i j}(t)\right)
$$

onde $\mathbf{F}_{n, i j}$ e $\mathbf{F}_{t, i j}$ são as componentes normal e tangencial da força de contato entre as partículas $i$ e $j$.

O momento total das forças de contato atuando na partícula $i$ é calculado por:

$$
\mathbf{T}^{(i)}(t)=\sum_{j=1}^{N}\left(\mathbf{R}^{(i)} \times \mathbf{F}_{t, i j}(t)\right)
$$

A partir da Eq. (4.7), as componentes normal e tangencial da força de contato, $\mathbf{F}_{c}^{(i)}$, são decomposta em força conservativa $\left(\right.$ mola, $\mathbf{F}^{S}$ ) e força dissipativa (amortecedor, $\mathbf{F}^{D}$ ):

$$
\mathbf{F}_{n, i j}(t)=\mathbf{F}_{n, i j}^{S}+\mathbf{F}_{n, i j}^{D}
$$




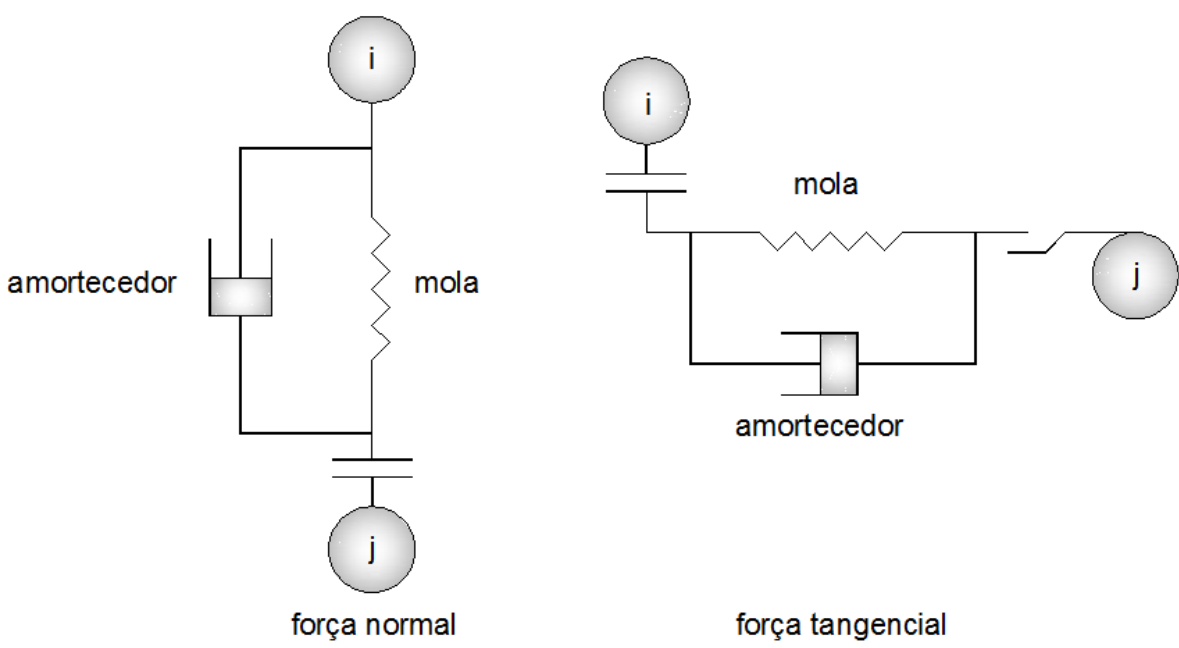

Figura 15 - Força de contato: sistema massa-mola-amortecedor nas direções normal e tangencial (TSUJI; TANAKA; ISHIDA, 1992)

$$
\mathbf{F}_{t, i j}(t)=\mathbf{F}_{t, i j}^{S}+\mathbf{F}_{t, i j}^{D}
$$

Considerando a Lei de Atrito de Coulomb entre as partículas, se a condição $\left|\mathbf{F}_{t, i j}\right|>\mu\left|\mathbf{F}_{n, i j}\right|$ é satisfeita durante o tempo de contato então ocorre o deslizamento entre as partículas e a força de contato tangencial é calculada por:

$$
\mathbf{F}_{t, i j}=-\mu\left|\mathbf{F}_{n, i j}\right| \mathbf{t}_{i j}
$$

onde $\mu$ é o coeficiente de atrito dinâmico.

\subsection{Força de contato: modelo linear}

No modelo linear o sistema massa-mola-amortecedor é linear. A força elástica é descrita pela Lei de Hooke $(F(\delta)=k \delta)$ e a força dissipativa é proporcional à velocidade. A força de mola normal $\left(\mathbf{F}_{n, i j}^{S}\right)$ e a força de mola tangencial $\left(\mathbf{F}_{t, i j}^{S}\right)$ são calculadas com base na sobreposição entre a colisão das partículas:

$$
\begin{gathered}
\mathbf{F}_{n, i j}^{S}=-k_{n} \delta_{n} \mathbf{n}_{i j} \\
\mathbf{F}_{t, i j}^{S}=-k_{t} \delta_{t} \mathbf{t}_{i j}
\end{gathered}
$$

onde $k_{n}$ e $k_{t}$ são a rigidez da mola nas direções normal e tangencial, respectivamente, $\delta_{n}$ é a sobreposição normal entre as partículas $i$ e $j$ e $\delta_{\mathbf{t}}$ é a deformação tangencial entre as partículas $i$ e $j$ (ver p. ex. Garg et al. (2012)). 
As forças dissipativas normal $\left(\mathbf{F}_{n, i j}^{D}\right)$ e tangencial $\left(\mathbf{F}_{t, i j}^{D}\right)$ são calculadas em função das componentes normal e tangencial da velocidade relativa de contato:

$$
\begin{aligned}
& \mathbf{F}_{n, i j}^{D}=-\eta_{n} \mathbf{V}_{n, i j} \\
& \mathbf{F}_{t, i j}^{D}=-\eta_{t} \mathbf{V}_{t, i j}
\end{aligned}
$$

onde $\eta_{n}$ e $\eta_{t}$ são os coeficientes de amortecimento nas direções normal e tangencial, respectivamente.

Considerando a direção normal, para um sistema com duas partículas de massas $m_{i}$ e $m_{j}$ colidindo sob ação das forças de contatos normais (Eq.(4.9) e aplicando a Segunda Lei de Newton (direção normal), escreve-se uma equação de segunda ordem para o movimento de colisão das partículas como:

$$
m_{e f f} \ddot{\delta}_{n}+\eta_{n} \dot{\delta}_{n}+k_{n} \delta_{n}=0
$$

onde $m_{\text {eff }}$ é a massa efetiva e $1 / m_{\text {eff }}=1 / m_{i}+1 / m_{j}$. Considerando as condições inicias $\delta_{n}(0)=0$ e $\dot{\delta}_{n}(0)=v_{0}$, onde $v_{0}$ é a velocidade de impacto, determina-se a solução da Eq. (4.16) para o caso sub-amortecido $(0 \leq \zeta<1)$, como:

$\left.\delta_{n}(t)=\left(v_{0} / \sqrt{k_{n} / m_{e f f}-\eta_{n}^{2} /\left(4 m_{\text {eff }}^{2}\right)}\right) \exp \left(-\left(\eta_{n} /\left(2 m_{\text {eff }}\right)\right) t\right) \sin \left(\sqrt{k_{n} / m_{\text {eff }}-\eta_{n}^{2} /\left(4 m_{\text {eff }}^{2}\right)}\right) t\right)$

onde $\zeta=\eta_{n} /\left(2 \sqrt{m_{e f f} k_{n}}\right)$ é a razão de amortecimento do sistema.

No impacto a sobreposição no final do contato é nula e o coeficiente de restituição representa a relação entre o impulso de restituição e de deformação, de forma que $\delta_{n}=0$ no instante $t=t_{c, n}$ e $\dot{\delta}_{n}\left(t_{c, n}\right)=-e_{n} \dot{\delta}_{n}(0)$, onde $t=t_{c, n}$ é o instante final do impacto e $e_{n}$ é o coeficiente de restituição normal no contato das partículas. Substituindo essas condições na Eq. (4.17) obtém-se expressões para $e_{n}$ e $t_{c, n}$ (ver p.ex. Shäfer, Dippel e Wolf (1996)):

$$
\begin{gathered}
e_{n}=\exp \left(-\frac{\eta_{n}}{2 m_{e f f}} t_{c, n}\right) \\
t_{c, n}=\pi\left(\frac{k_{n}}{m_{\text {eff }}}-\frac{\eta_{n}^{2}}{4 m_{\text {eff }}^{2}}\right)^{-1 / 2}
\end{gathered}
$$

A partir da Eq. (4.18) e da Eq. (4.19) $k_{n}$ e $\eta_{n}$ são calculados por:

$$
k_{n}=\frac{m_{e f f}}{t_{c, n}^{2}}\left(\ln ^{2} e_{n}+\pi^{2}\right)
$$




$$
\eta_{n}=-2 \sqrt{m_{e f f} k_{n}} \frac{\ln e_{n}}{\sqrt{\ln ^{2} e_{n}+\pi^{2}}}=-2 \frac{m_{e f f}}{t_{c, n}} \ln e_{n}
$$

Se os valores de $e_{n}$ e $t_{c, n}$ são conhecidos de medidas experimentais, as quantidades $k_{n}$ e $\eta_{n}$ podem ser determinadas pelas Eq. (4.20) e Eq. (4.21), respectivamente. O usual é conhecer somente o valor de $e_{n}$, de forma que é necessário um procedimento para estimar o valor de $k_{n}$ no modelo linear ou o valor desse parâmetro pode ser assumido de experimentos anteriores. Navarro e Braun (2013) apresentam um método para estimar a rigidez da mola usando analogia com modelos não-lineares (ver Seção 4.3). Sun, Battaglia e Subramaniam (2007) comentam que se o valor da rigidez da mola for grande evita-se sobreposição exagerada das partículas. Entretanto se o valor for muito alto o tempo de contato é pequeno assemelhando-se ao contato "instantâneo" do tipo "esfera-dura".

A seguir, o movimento da partícula na direção normal é detalhado em termos de parâmetros dinâmicos. A equação de movimento para a sobreposição na direção normal, $\delta_{n}$, descrevendo a colisão de duas partículas é reescrita a partir da Eq.(4.16) como:

$$
\ddot{\delta}_{n}+2 \Psi \dot{\delta}_{n}+\Omega_{0}^{2} \delta_{n}=0
$$

onde $\Omega_{0}=\sqrt{k_{n} / m_{e f f}}$ é a frequência natural do oscilador não-amortecido e $\Psi=\eta_{n} /\left(2 m_{e f f}\right)$ é o coeficiente de amortecimento responsável pela dissipação de energia.

A solução da equação diferencial do oscilador amortecido, Eqs. (4.16), (4.22), para o caso sub-amortecido $\left(\Omega_{0}>\Psi\right)$, com condições iniciais $\delta_{n}(0)=0$ e $\dot{\delta}_{n}(0)=v_{0}$ é expressa por:

$$
\delta_{n}(t)=\left(v_{0} / \Omega\right) \exp (-\Psi t) \sin (\Omega t)
$$

Diferenciando a Eq. (4.23) em relação ao tempo obtém-se:

$$
\dot{\delta}_{n}(t)=\left(v_{0} / \Omega\right) \exp (-\Psi t)(-\Psi \sin (\Omega t)+\Omega \cos (\Omega t))
$$

onde $v_{0}$ é a velocidade relativa inicial de impacto e $\Omega=\sqrt{\Omega_{0}^{2}-\Psi^{2}}$ é a freqüência natural amortecida do oscilador. O modelo linear massa-mola-amortecedor é fácil de ser utilizado devido à existência de solução analítica para a sobreposição (Eq. 4.23) e de relação entre os parâmetros $k_{n}$ e $\eta_{n}$ (Eq. 4.26). O tempo de contato entre as partículas é determinado a partir da substituição da condição $\delta_{n}\left(t_{c, n}\right)=0$ na Eq. (4.23) o que resulta em $t_{c, n}=\pi / \Omega$ (ou Eq. 4.19). A velocidade relativa imediatamente após o término do contato entre as 
partículas é igual a $\dot{\delta}_{n}\left(t_{c, n}\right)=-v_{0} \exp \left(-\Psi t_{c, n}\right)$. O coeficiente de restituição na direção normal é dado pela Eq. (4.18) ou

$$
e_{n}=-\frac{\dot{\delta}_{n}\left(t_{c, n}\right)}{\dot{\delta}_{n}(0)}=\exp (-\pi \Psi / \Omega)
$$

Invertendo a Eq. (4.25) calcula-se o coeficiente de amortecimento $\Psi$ ou $\eta_{n}$, ver Eq. (4.21), em função do coeficiente $e_{n}$ :

$$
\Psi=\frac{-\ln e_{n}}{\sqrt{\ln ^{2} e_{n}+\pi^{2}}} \Omega_{0}
$$

No modelo linear massa-mola-amortecedor o coeficiente de restituição normal $e_{n} \mathrm{e}$ o tempo de contato $t_{c, n}$ são independentes da velocidade de impacto $v_{0}$ (ver Eqs. $4.25 \mathrm{e}$ 4.19). A sobreposição máxima é derivada a partir da condição $\dot{\delta}_{n}\left(t_{\max }\right)=0$, e das Eqs. $(4.23)$ e $(4.24)$ :

$$
\delta_{\text {max }}=\left(v_{0} / \Omega_{0}\right) \exp \left(-\frac{\arctan (\beta)}{\beta}\right)
$$

onde $\beta=-\Omega / \Psi=\pi / \ln e_{n}$ para $0<e_{n}<1.0$. O tempo de contato é reescrito como:

$$
t_{c, n}=\frac{\pi}{\Omega_{0}} \sqrt{\left(1+\frac{1}{\beta^{2}}\right)}
$$

Integrando a Eq. (4.23) durante o tempo de contato, a sobreposição média $\bar{\delta}_{n}$ é expressa por:

$$
\bar{\delta}_{n}=\frac{1}{t_{c, n}} \int_{0}^{t_{c, n}} \delta_{n}(t)=\frac{v_{0}}{\pi \Omega_{0}} \frac{-\beta}{\sqrt{1+\beta^{2}}}\left(1+\exp \left(\frac{\pi}{\beta}\right)\right)
$$

Para uma colisão perfeitamente elástica $\left(e_{n}=1.0\right), \Psi=\eta_{n}=0$, a sobreposição máxima, o tempo de contato e a sobreposição média são caculados por $\delta_{\max }=v_{0} / \Omega_{0}$, $t_{c, n}=\pi / \Omega_{0}$ e $\bar{\delta}_{n}=2 v_{0} /\left(\pi \Omega_{0}\right)$, respectivamente.

Utilizando os seguintes parâmetros adimensionais na Eq. (4.22):

$$
\delta_{n}^{*}=\frac{\delta_{n}}{v_{0}} \Omega_{0}, \quad \delta_{n}^{* \prime}=\frac{\dot{\delta}_{n}}{v_{0}}, \quad t^{*}=t \Omega_{0}
$$

a equação do movimento para a sobreposição adimensional na direção normal, $\delta_{n}^{*}$, pode ser escrita como:

$$
\delta_{n}^{* \prime \prime}+2 \nu^{*} \delta_{n}^{* \prime}+\delta_{n}^{*}=0
$$


onde as aspas denotam as derivadas em relação ao tempo adimensional $t^{*}$. O parâmetro $\nu^{*}$ é o coeficiente de amortecimento adimensional dado por $\left(0<e_{n} \leq 1\right)$ :

$$
\nu^{*}=\frac{\Psi}{\Omega_{0}}=\frac{-\ln e_{n}}{\sqrt{\ln ^{2} e_{n}+\pi^{2}}}
$$

onde $0 \leq \nu^{*}<1$. A solução da equação da sobreposição adimensional (Eq. 4.31) para o caso subamortecido $\left(0 \leq \nu^{*}<1\right)$ com condições iniciais $\delta_{n}^{*}(0)=0$ e $\delta_{n}^{* \prime}(0)=1$ é dada por

$$
\delta_{n}^{*}\left(t^{*}\right)=\left(1 / \Omega^{*}\right) \exp \left(-\nu^{*} t^{*}\right) \sin \left(\Omega^{*} t^{*}\right)
$$

onde $\Omega^{*}=\sqrt{1-\nu^{* 2}}$ é a freqüência adimensional do oscilador amortecido. A sobreposição máxima e a duração do contato adimensionais são expressas por:

$$
\begin{gathered}
\delta_{\text {max }}^{*}=\exp \left(-\frac{\arctan \left(\beta^{*}\right)}{\beta^{*}}\right) \\
t_{c, n}^{*}=\pi \sqrt{\left(1+\frac{1}{\beta^{* 2}}\right)}
\end{gathered}
$$

onde $\beta^{*}=-\Omega^{*} / \nu^{*}=\pi / \ln e_{n}=\beta$, para $0<e_{n}<1.0$. Para as colisões perfeitamente elásticas $\left(e_{n}=1.0\right)$, o coeficiente de amortecimento adimensional é igual a zero, e neste caso, a sobreposição máxima e a duração do contato adimensionais são, respectivamente, $\delta_{\max }^{*}=1.0$ e $t_{c, n}^{*}=\pi$.

A Figura 16 ilustra um conjunto de curvas para a sobreposição adimensional, $\delta^{*}$, versus o tempo adimensional para diversos valores do coeficiente de restituição $\left(e_{n}=\right.$ $0.9,0.7,0.5,0.3,0.1)$. Com essas curvas pode-se estimar a duração do contato. Nessa figura a duração do contato aumenta com o decréscimo do coeficiente de restituição (Tabela 9). A sobreposição máxima também aumenta com o coeficiente de restituição até o caso da colisão perfeitamente elástica (Tabela 9). Valores altos para a sobreposição caracterizam a hipótese do modelo de esfera-suave baseado em partículas geometricamente rígidas (limitadas para pequenas deformações ou sobreposições) menos acurado podendo causar erros físicos nesse modelo devido aos efeitos de exclusão dos volumes sobrepostos. No modelo linear, a duração do contato (ver Eqs. 4.19, 4.28, 4.35), independe da velocidade de impacto, oposto o que ocorre nas colisões reais (ver (GOLDSMITH, 1960), (LUN; SAVAGE, 1986)).

Na Figura 17, no final do contato, de acordo com a Eq. (4.25), o coeficiente de restituição tem o valor negativo da velocidade no final do impacto, isto é, $e_{n}=-\delta_{n}^{* \prime}\left(t_{c, n}^{*}\right)$. A Figura 17 e a Eq. (4.25) mostram que o coeficiente de restituição é independente da velocidade de impacto. Para colisões reais, $e_{n}$ decresce com o aumento da velocidade $v_{0}$.

Observa-se na Figura 18 que a força de contato é descontínua entre o início e o final do contato, $\left(a^{*}(0) \neq a^{*}\left(t_{c, n}^{*}\right) \neq 0\right)$ devido as forcas de amortecimento fazendo com que 
a aceleração nem inicia e nem termina com o valor nulo. Nos contatos reais essas forças são contínuas. Observa-se que na região do final do contato as forças são coesivas $\left(a^{*}>0\right)$ sendo nos contatos reais elas são sempre repulsivas $\left(a^{*}<0\right)$ para sistema sem coesão.

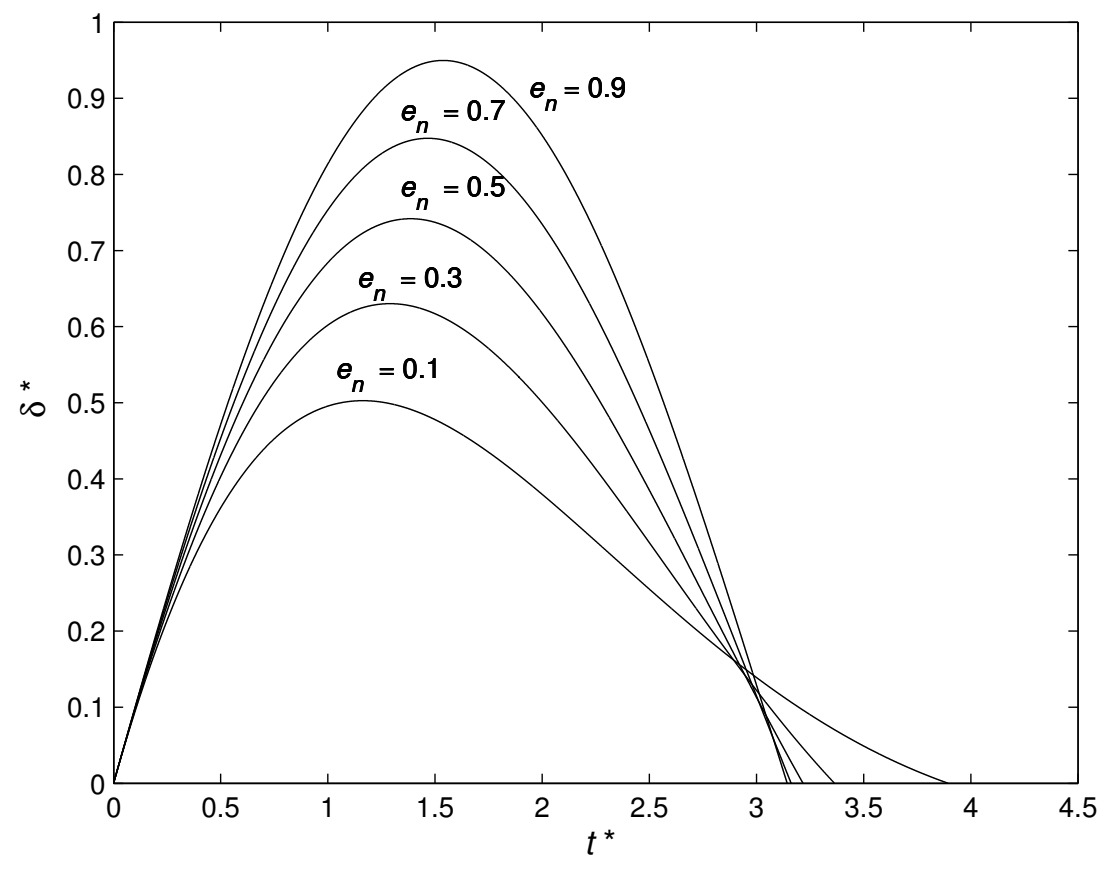

Figura 16 - Sobreposição adimensional (modelo linear).

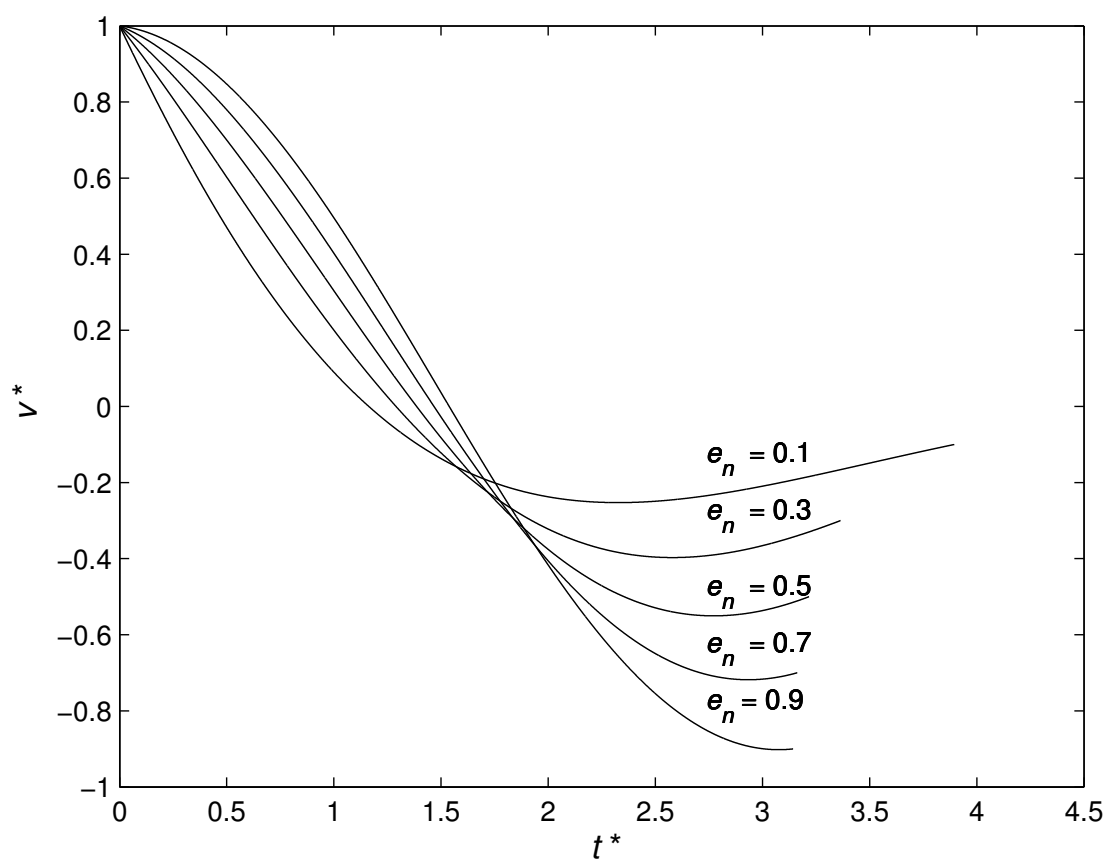

Figura 17 - Velocidade adimensional (modelo linear). 


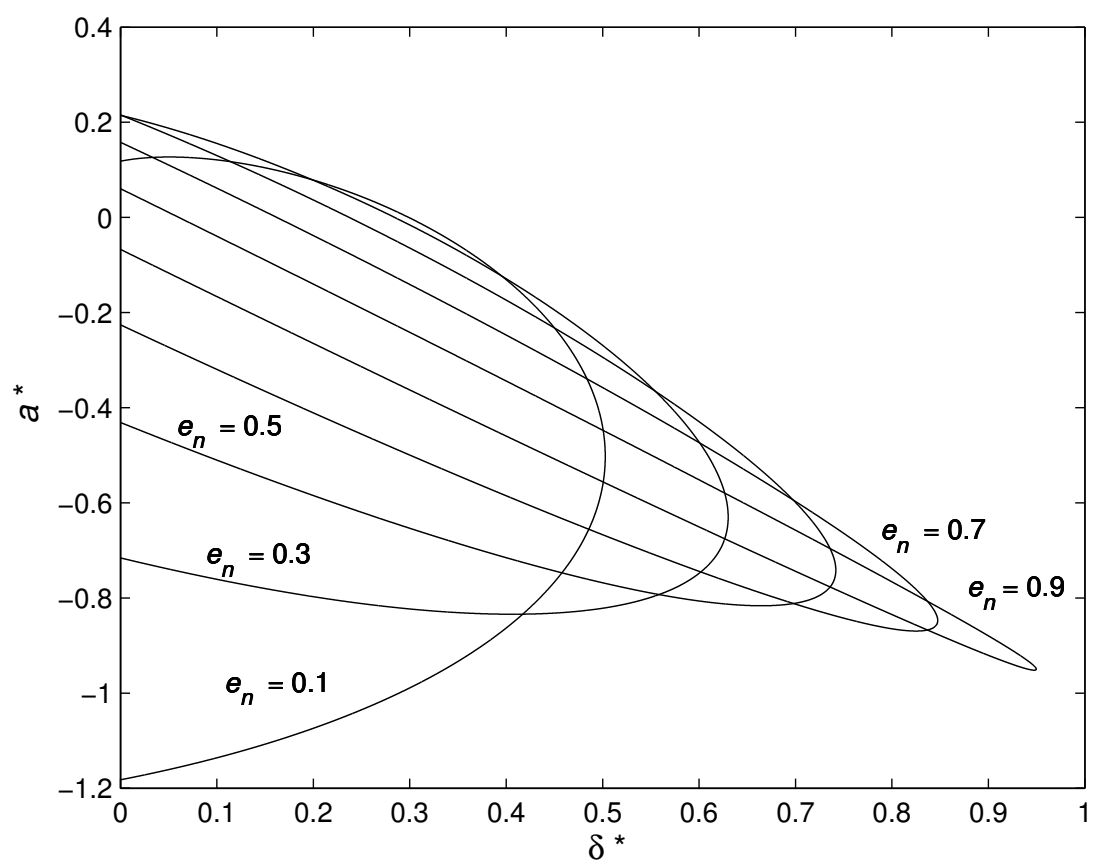

Figura 18 - Aceleração adimensional (modelo linear).

A Tabela 9 mostra, para diversos valores do coeficiente de restituição normal $\left(e_{n}=1.0,0.9,0.7,0.5,0.3,0.1\right)$, os seguintes parâmetros adimensionais: coeficiente de amortecimento $\nu^{*}$, instante na sobreposição máxima $t_{\text {max }}^{*}$, sobreposição máxima $\delta_{\text {max }}^{*}$, duração do contato $t_{c, n}^{*}$, aceleração inicial $a^{*}(0)$, e aceleração no instante final do contato $a^{*}\left(t_{c, n}^{*}\right)$, respectivamente. Observa-se que a força de contato é descontínua no início e no fim do contato entre os corpos $\left(a^{*}(0) \neq a^{*}\left(t_{c, n}^{*} \neq 0\right)\right.$, isto é, a aceleração não começa e termina no mesmo valor na presença de amortecimento no modelo linear. Como descrito anteriormente, a força de contato é coesiva $\left(a^{*}>0\right)$ no final do impacto, sendo que para colisões reais, a força de contato é sempre repulsiva $\left(a^{*}<0\right)$.

Tabela 9 - Parâmetros adimensionais para o modelo linear.

\begin{tabular}{lllllll}
\hline$e_{n}$ & $\nu^{*}$ & $t_{\max }^{*}$ & $\delta_{\max }^{*}$ & $t_{c, n}^{*}$ & $a^{*}(0)$ & $a^{*}\left(t_{c, n}^{*}\right)$ \\
\hline 1 & 0 & 1.5708 & 1 & 3.1416 & 0 & 0 \\
0.9 & 0.0335 & 1.5381 & 0.9498 & 3.1434 & -0.0670 & 0.0603 \\
0.7 & 0.1128 & 1.4671 & 0.8475 & 3.1618 & -0.2256 & 0.1579 \\
0.5 & 0.2155 & 1.3862 & 0.7418 & 3.2172 & -0.4309 & 0.2155 \\
0.3 & 0.3579 & 1.2903 & 0.6302 & 3.3644 & -0.7157 & 0.2147 \\
0.1 & 0.5912 & 1.1635 & 0.5027 & 3.8951 & -1.1823 & 0.1182 \\
\hline
\end{tabular}

Na direção tangencial, de acordo com Shäfer, Dippel e Wolf (1996), a rigidez da mola na direção tangencial é assumida ser igual a dois sétimos da rigidez normal $\left(k_{t}=2 / 7 k_{n}\right)$. O coeficiente de amortecimento tangencial é calculado como a metade do coeficiente na direção normal, isto é, $\eta_{t}=0.5 \eta_{n}$ ((SILBERT et al., 2001), (SILBERT; LANDRY; GREST, 
2003)).

\subsection{Força de contato: modelo não-linear}

A colisão entre corpos pode ser descrita através da teoria de contato Hertziana, ver Hertz (1882), Goldsmith (1960), Johnson (1985). A colisão entre dois corpos elásticos é modelada pela integração da Lei de de Hooke sobre a área deformada e resulta em uma relação não-linear conhecida por Lei de Hertz $\left(F\left(\delta_{n}\right)=k_{n, H z} \delta_{n}^{3 / 2}\right)($ HERTZ, 1882). As forças normal e tangencial, $\left(\mathbf{F}_{n, i j}^{S}\right)$ e $\left(\mathbf{F}_{t, i j}^{S}\right)$, são dadas por (TSUJI; TANAKA; ISHIDA, 1992):

$$
\begin{gathered}
\mathbf{F}_{n, i j}^{S}=-k_{n, H z} \delta_{n}{ }^{\frac{1}{2}} \delta_{n} \mathbf{n}_{i j}=-k_{n, H z} \delta_{n}{ }^{\frac{3}{2}} \mathbf{n}_{i j} \\
\mathbf{F}_{t, i j}^{S}=-k_{t, H z} \delta_{n}{ }^{\frac{1}{2}} \delta_{\mathbf{t}}
\end{gathered}
$$

onde o coeficiente de rigidez da mola nas direções normal, $k_{n, H z}$, e tangencial, $k_{t, H z}$, durante o contato entre os corpos $i$ e $j$, é calculado a partir do módulo de Young e do coeficiente de Poisson por:

$$
\begin{aligned}
& k_{n, H z}=\frac{4}{3} E_{e f f} \sqrt{r_{e f f}} \\
& k_{t, H z}=\frac{16}{3} G_{e f f} \sqrt{r_{e f f}}
\end{aligned}
$$

e $E_{e f f}, G_{e f f}$ e $r_{e f f}$ são, respectivamente, o módulo de Young efetivo, o módulo de cisalhamento efetivo e o raio efetivo dos corpos (partículas esféricas de raios $r_{i}$ e $r_{j}$ ), calculados por:

$$
\begin{gathered}
E_{e f f}=\left[\left(1-\sigma_{i}^{2}\right) / E_{i}+\left(1-\sigma_{j}^{2}\right) / E_{j}\right]^{-1} \\
G_{e f f}=\left[\left(2-\sigma_{i}\right) / G_{i}+\left(2-\sigma_{j}\right) / G_{j}\right]^{-1} \\
r_{e f f}=\left[1 / r_{i}+1 / r_{j}\right]^{-1}
\end{gathered}
$$

$E_{i}$ e $E_{j}$ são os módulos de Young, $\sigma_{i}$ e $\sigma_{j}$ são os coeficientes de Poisson, e $G_{i}$ e $G_{j}$ são os módulos de cisalhamento para os corpos $i$ e $j$, respectivamente. Os módulos de cisalhamento são dados por:

$$
\begin{gathered}
G_{i}=E_{i} / 2\left(1+\sigma_{i}\right) \\
G_{j}=E_{j} / 2\left(1+\sigma_{j}\right)
\end{gathered}
$$

A relação entre a força tangencial (Eq. 4.37) e a sobreposição tangencial foi derivada por Mindlin (1949) e Mindlin e Deresiewicz (1953). De acordo com a teoria desses autores a relação entre força-deslocamento depende da sobreposição normal, $\delta_{n}$. 
Hunt e Grossley (1975) derivaram uma expressão para o termo de amortecimento $\lambda \delta_{n}(t)^{p} \dot{\delta}_{n}(t)^{q}$. No presente trabalho é assumido que $q=1$, mas como discutido por (HUNT; GROSSLEY, 1975) outras escolhas são possíveis para o índice $q$. Considerando os autores Hunt e Grossley (1975) é possível escrever a seguinte expressões para as forças de amortecimento não-linear, nas direções normal e tangencial, respectivamente:

$$
\begin{aligned}
& \mathbf{F}_{n, i j}^{D}=-\eta_{n, H z} \delta_{n}{ }^{p} \mathbf{V}_{n, i j} \\
& \mathbf{F}_{t, i j}^{D}=-\eta_{t, H z} \delta_{n}{ }^{p} \mathbf{V}_{t, i j}
\end{aligned}
$$

Tsuji, Tanaka e Ishida (1992) consideraram $p=1 / 4$ e propuseram uma expressão para o cálculo das forças dissipativas, isto é, as forças de amortecimento normais e tangenciais, $\left(\mathbf{F}_{n, i j}^{D}\right)$ e $\left(\mathbf{F}_{t, i j}^{D}\right)$, sendo descritas por:

$$
\begin{aligned}
& \mathbf{F}_{n, i j}^{D}=-\eta_{n, H z} \delta_{n}{ }^{1 / 4} \mathbf{V}_{n, i j} \\
& \mathbf{F}_{t, i j}^{D}=-\eta_{t, H z} \delta_{n}{ }^{1 / 4} \mathbf{V}_{t, i j}
\end{aligned}
$$

sendo que os coeficientes de amortecimento normal e tangencial, $\eta_{n, H z}, \eta_{t, H z}$, são relacionados com a rigidez não-linear da mola e com o coeficiente de restituição do seguinte modo (TSUJI; TANAKA; ISHIDA, 1992):

$$
\begin{aligned}
\eta_{n, H z} & =-\sqrt{5} \sqrt{m_{e f f} k_{n, H z}} \frac{\ln e_{n}}{\sqrt{\ln ^{2} e_{n}+\pi^{2}}} \\
\eta_{t, H z} & =-\sqrt{5} \sqrt{m_{e f f} k_{t, H z}} \frac{\ln e_{t}}{\sqrt{\ln ^{2} e_{t}+\pi^{2}}}
\end{aligned}
$$

A equação do movimento para a sobreposição na direção normal é expressa pela equação Hertziana não-linear de um oscilador amortecido:

$$
m_{e f f} \ddot{\delta}_{n}+\eta_{n, H z} \delta_{n}^{1 / 4} \dot{\delta}_{n}+k_{n, H z} \delta_{n}^{3 / 2}=0
$$

Substituindo os seguintes parâmetros adimensionais na Eq. (4.51):

$$
\delta_{n}^{*}=\delta_{n}\left(\frac{k_{n, H z}}{v_{0}^{2} m_{e f f}}\right)^{2 / 5}, \quad \delta_{n}^{* \prime}=\frac{\dot{\delta}_{n}}{v_{0}}, \quad t^{*}=t\left(\frac{k_{n, H z} v_{0}^{1 / 2}}{m_{e f f}}\right)^{2 / 5}
$$

onde $v_{0}$ é a velocidade de impacto, a equação de movimento para a sobreposição adimensional na direção normal, $\delta_{n}^{*}$, é expressa por:

$$
\delta_{n}^{* \prime \prime}+2 \nu_{A}^{*} \delta_{n}^{* 1 / 4} \delta_{n}^{* \prime}+\delta_{n}^{* 3 / 2}=0
$$


onde as aspas denotam as derivadas em relação ao tempo adimensional $t^{*}$. O parâmetro $\nu_{A}^{*}$ representa o coeficiente de amortecimento adimensional e é dado por:

$$
\nu_{A}^{*}=\frac{\eta_{n, H z}}{2 \sqrt{\left(m_{e f f} k_{n, H z}\right)}}
$$

A equação (4.53) pode ser resolvida numericamente com condições iniciais $\delta_{n}^{*}(0)=0$ e $\delta_{n}^{* \prime}(0)=1$ para determinar o histórico no tempo das sobreposições adimensionais, isto é, sobreposição $\delta_{n, i}^{*}$ em função dos instantes $t_{i}^{*}$. Utilizando algorítimos computacionais a sobreposição máxima, a duração de contato e a sobreposição média adimensionais podem ser determinados, respectivamente, por:

$$
\begin{gathered}
\delta_{\text {max }}^{*}=\max _{i}\left\{\delta_{n, i}^{*}\left(t_{i}^{*}\right)\right\} \\
\delta_{n, i}^{*}\left(t_{i}^{*}\right)=0, \quad t_{c, n}^{*}=t_{i}^{*} \\
\bar{\delta}_{n}^{*}=\frac{1}{t_{c, n}^{*}} \sum_{0}^{t_{c, n}^{*}}\left\{\delta_{n, i}^{*}\right\}
\end{gathered}
$$

De forma similar que as Figuras 16, 17, 18, as Figuras 19, 20, 21 ilustram a evolução da sobreposição adimensional para o modelo não-linear (Eq. 4.51). As figuras apresentam diversas curvas para valores de amortecimento $\left(\nu_{A}^{*}=0,0.1,0.25,0.4,0.6\right)$ em função do tempo adimensional. Como no caso linear, os gráficos foram plotados até o final do contato $t_{c, n}^{*}$. Observa-se na Figura 19 que a duração do impacto aumenta e a sobreposição máxima diminui com o incremento do valor do amortecimento $\nu_{A}^{*}$. No final do impacto na Figura 20 pode-se determinar o coeficiente de restituição normal $e_{n}$ que é independente da velocidade de impacto (ver Eq. 4.54). Observa-se na Figura 21 que a força é descontínua entre o início e o final do contato. A descontinuidade na força de contato nesse modelo não-linear é menor do que a comparada com o modelo linear (ver Figura 18). Esse fato também é observado nas Tabelas 9 e 10.

A Tabela 10 mostra, para diversos valores do coeficiente de amortecimento, $\left(\nu_{A}^{*}\right)$, os seguintes parâmetros adimensionais: coeficiente de restituição normal $e_{n}$, instante na sobreposição máxima $t_{\text {max }}^{*}$, sobreposição máxima $\delta_{\text {max }}^{*}$, sobreposição média $\bar{\delta}_{n}^{*}$, duração do contato $t_{c, n}^{*}$, aceleração inicial $a^{*}(0)$, e aceleração no final do impacto $a^{*}\left(t_{c, n}^{*}\right)$, respectivamente. Esses parâmetros podem ser calculados a partir de um conjunto de pontos de sobreposição para diversos instantes (Eqs. 4.55, 4.56 e 4.57). O coeficiente de restituição normal é dado por $e_{n}=-\delta_{n}^{* \prime}\left(t_{c, n}^{*}\right)$ e a aceleração por $a^{*}=v^{* \prime}$. 


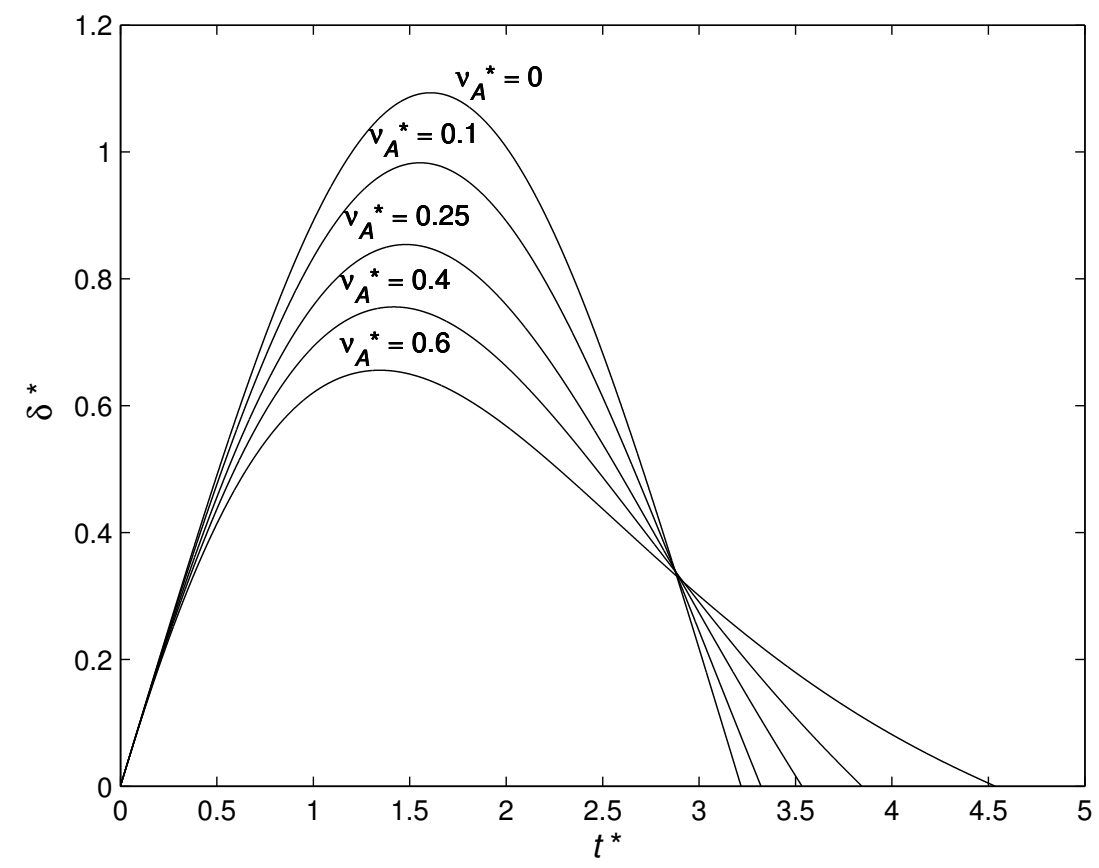

Figura 19 - Sobreposição adimensional (modelo não-linear, Eq. (4.53)).

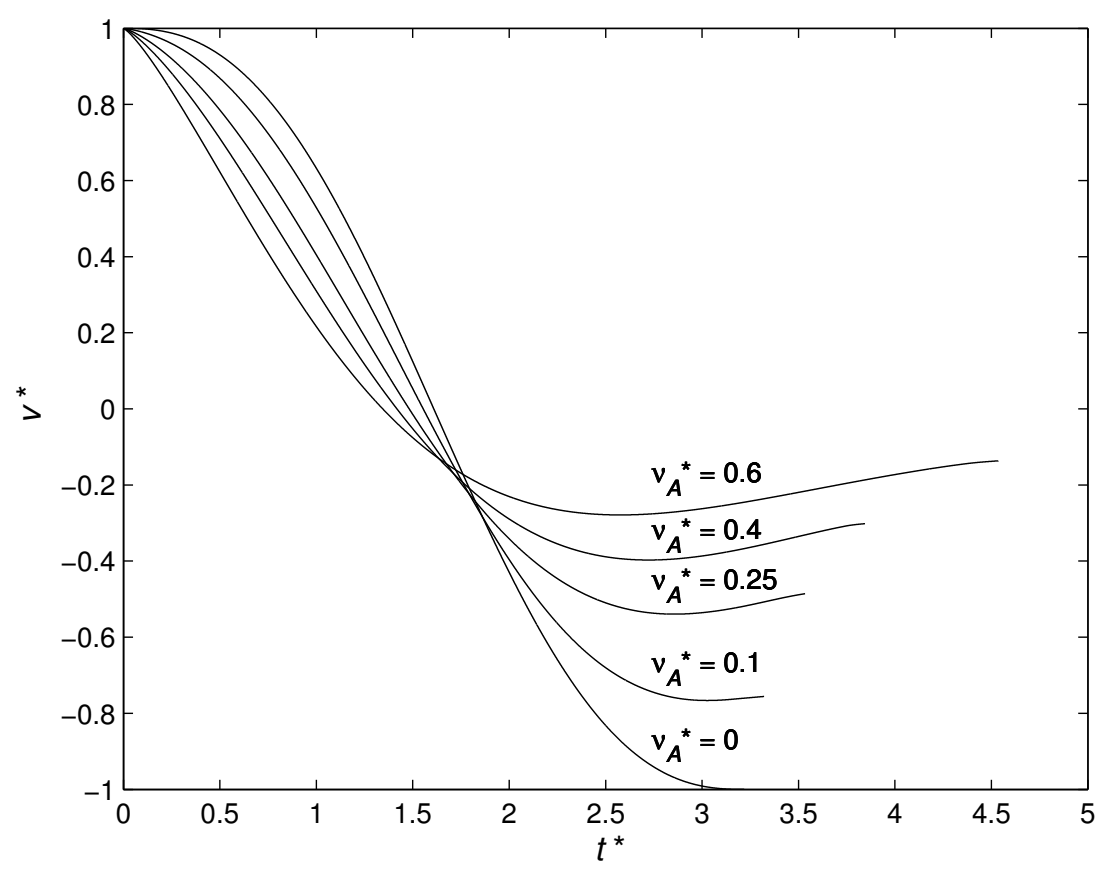

Figura 20 - Velocidade adimensional (modelo não-linear, Eq. (4.53)).

Uma expressão para valores de um parâmetro $\alpha$ plotado no trabalho de Tsuji, Tanaka e Ishida (1992) é apresentada por Antypov e Elliott (2011) e reescrita para o 


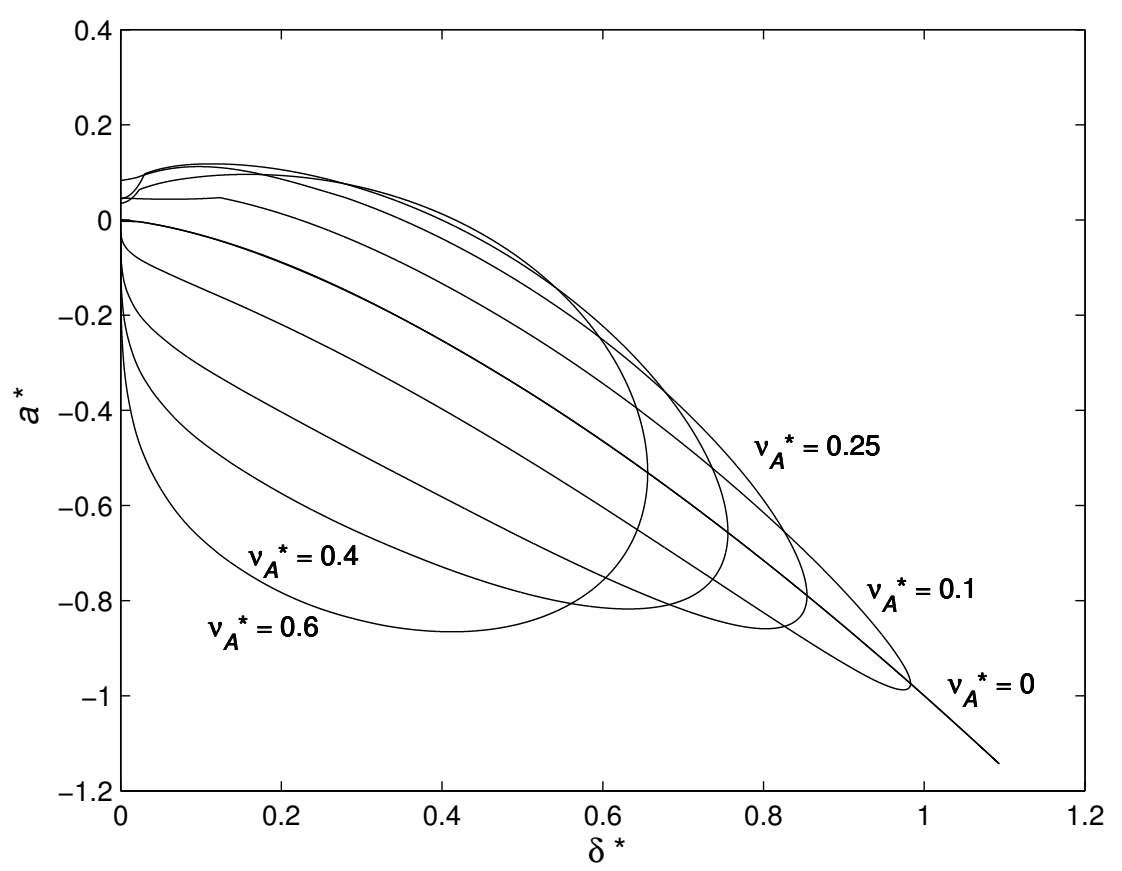

Figura 21 - Aceleração adimensional (modelo não-linear, Eq. (4.53)).

Tabela 10 - Parâmetros adimensionais para o modelo não-linear (4.53).

\begin{tabular}{llllllll}
\hline$\nu_{A}^{*}$ & $e_{n}$ & $t_{\max }^{*}$ & $\delta_{\max }^{*}$ & $\bar{\delta}_{n}^{*}$ & $t_{c, n}^{*}$ & $a^{*}(0)$ & $a^{*}\left(t_{c, n}^{*}\right)$ \\
\hline 0 & 1.0000 & 1.6090 & 1.0936 & 0.6833 & 3.2181 & 0 & 0 \\
0.02 & 0.9453 & 1.5976 & 1.0693 & 0.6682 & 3.2366 & -0.0018 & 0.0034 \\
0.04 & 0.8936 & 1.5864 & 1.0465 & 0.6537 & 3.2562 & -0.0036 & 0.0050 \\
0.06 & 0.8446 & 1.5754 & 1.0243 & 0.6396 & 3.2768 & -0.0054 & 0.0061 \\
0.08 & 0.7982 & 1.5647 & 1.0032 & 0.6260 & 3.2985 & -0.0072 & 0.0032 \\
0.1 & 0.7542 & 1.5542 & 0.9830 & 0.6128 & 3.3213 & -0.0089 & 0.0089 \\
0.15 & 0.6536 & 1.5288 & 0.9358 & 0.5815 & 3.3836 & -0.0134 & 0.0107 \\
0.2 & 0.5648 & 1.5046 & 0.8931 & 0.5524 & 3.4540 & -0.0179 & 0.0100 \\
0.25 & 0.4864 & 1.4815 & 0.8542 & 0.5251 & 3.5338 & -0.0224 & 0.0116 \\
0.3 & 0.4168 & 1.4595 & 0.8185 & 0.4993 & 3.6243 & -0.0268 & 0.0122 \\
0.35 & 0.3550 & 1.4383 & 0.7858 & 0.4748 & 3.7273 & -0.0313 & 0.0097 \\
0.4 & 0.3001 & 1.4181 & 0.7557 & 0.4514 & 3.8449 & -0.0358 & 0.0096 \\
0.45 & 0.2513 & 1.3987 & 0.7279 & 0.4288 & 3.9801 & -0.0402 & 0.0086 \\
0.5 & 0.2079 & 1.3801 & 0.7022 & 0.4069 & 4.1368 & -0.0447 & 0.0076 \\
0.55 & 0.1695 & 1.3621 & 0.6782 & 0.3853 & 4.3200 & -0.0492 & 0.0049 \\
0.6 & 0.1356 & 1.3449 & 0.6559 & 0.3639 & 4.5368 & -0.0536 & 0.0057 \\
0.7 & 0.0803 & 1.3122 & 0.6156 & 0.3205 & 5.1163 & -0.0626 & 0.0036 \\
0.8 & 0.0400 & 1.2818 & 0.5801 & 0.2737 & 6.0353 & -0.0715 & 0.0017 \\
0.9 & 0.0141 & 1.2533 & 0.5487 & 0.2186 & 7.7508 & -0.0805 & 0.0004 \\
\hline
\end{tabular}


coeficiente de amortecimento $\nu_{A}^{*}$ como $\left(e_{n}>0\right)$ :

$$
\nu_{A}^{*}=\frac{\alpha}{2}=-\frac{\sqrt{5}}{2} \frac{\ln e_{n}}{\sqrt{\ln ^{2} e_{n}+\pi^{2}}}
$$

Tsuji, Tanaka e Ishida (1992) e Antypov e Elliott (2011) deduziram um mapeamento entre o oscilador Hertiziano com força viscosa proporcional a potência 1/4 e o modelo massamola-amortecedor linear. Os autores demostraram que para um apropriado escalonamento dos eixos de coordenadas, ambos os modelos seguem a mesma trajetória. Baseando nos trabalhos de Tsuji, Tanaka e Ishida (1992) e Antypov e Elliott (2011), e desde que

$$
v^{*}\left(t^{*}\right)=\delta_{n}^{* \prime}=\frac{d \delta_{n}^{*}}{d t^{*}} \quad \delta_{n}^{* \prime \prime}=\frac{d \delta_{n}^{*}}{d t^{*}} \frac{d v^{*}}{d \delta_{n}^{*}}=v^{*} \frac{d v^{*}}{d \delta_{n}^{*}}
$$

a Eq. (4.53) pode ser reescrita como:

$$
v^{*} \frac{d v^{*}}{d \delta_{n}^{*}}+2 \nu_{A}^{*} \delta_{n}^{* 1 / 4} v^{*}+\delta_{n}^{* 3 / 2}=0
$$

Substituindo a função $\delta_{n}^{*}$ por uma função $x(t)$ tal que (ANTYPOV; ELLIOTT, 2011):

$$
\delta_{n}^{*}=A x^{n} \quad d \delta_{n}^{*}=n A x^{n-1} d x
$$

a Eq. (4.60) fica transformada em:

$$
v^{*} \frac{d v^{*} A^{-1} x^{1-n}}{n d x}+2 \nu_{A}^{*} A^{1 / 4} x^{n / 4} v^{*}+A^{3 / 2} x^{3 n / 2}=0
$$

Note que se $1-n=n / 4(n=4 / 5)$, as potências de $x$ são as mesmas para o primeiro e segundo termos. Dividindo a Eq. (4.62) por $x^{1 / 5}$ resulta em

$$
v^{*} \frac{5 d v^{*} A^{-1}}{4 d x}+2 \nu_{A}^{*} A^{1 / 4} v^{*}+A^{3 / 2} x=0
$$

Escolhendo um valor para $A$ em que os coeficientes dos primeiro e terceiro termos da Eq. (4.63) se tornam idênticos, isto é, $5 A^{-1} / 4=A^{3 / 2}$ ou $A=(5 / 4)^{2 / 5}$, a Eq. (4.63) é reescrita pela substituição do valor de $A$ e pela normalização através da divisão por $A=(5 / 4)^{3 / 5}$, tornando-se:

$$
v^{*} \frac{d v^{*}}{d x}+2 \nu_{A}^{*} \frac{2}{\sqrt{5}} v^{*}+x=0 .
$$

Assumindo que as velocidades permanecem inalteradas (ANTYPOV; ELLIOTT, 2011), isto é,

$$
v^{*}\left(t^{*}\right)=\hat{v}\left(\tau_{1}\right) \quad \hat{v}\left(\tau_{1}\right)=\frac{d x}{d \tau_{1}}=x^{\prime},
$$

pode-se reescrever a Eq. (4.64) como

$$
\hat{v} \frac{d \hat{v}}{d x}+2 \nu_{A}^{*} \frac{2}{\sqrt{5}} \hat{v}+x=x^{\prime \prime}+2 \nu_{A}^{*} \frac{2}{\sqrt{5}} x^{\prime}+x=0 .
$$


Comparando a Eq. (4.66) com a Eq. (4.31) do modelo linear, por similaridade, pode-se escrever:

$$
\nu_{A}^{*}=\frac{\sqrt{5}}{2} \nu^{*}
$$

Usando as Eqs. (4.67) e (4.32) resulta na Eq. (4.58) para o cálculo do coeficiente de amortecimento do modelo não-linear. Observa-se que esse procedimento descrito por Tsuji, Tanaka e Ishida (1992) e Antypov e Elliott (2011) reescalona a sobreposição ou deformação através da Eq. (4.61) da forma:

$$
\delta_{n}^{*}=(5 / 4)^{2 / 5}(x)^{4 / 5}
$$

Com os valores dos coeficientes de restituição, obtidos numericamente e mostrados na Tabela 10, pode-se obter, aproximadamente, os valores do coeficiente $\nu_{A}^{*}$ dessa mesma tabela usando a Eq. (4.58).

Os modelos linear e não-linear (Eqs. 4.16 e 4.51), respectivamente, resultam em um coeficiente de restituição independente da velocidade de impacto. Estudos experimentais realizados por Goldsmith (1960) mostram uma diminuição monotônica pequena de $e_{n}$ com a velocidade de impacto (GOLDSMITH, 1960; KUWABARA; KONO, 1987).

Como mencionado anteriormente Hunt e Grossley (1975) introduziram um termo para o cálculo do amortecimento da forma $\lambda \delta_{n}(t)^{p} \dot{\delta_{n}}(t)$, e consideraram o valor $p=3 / 2$ para uma esfera colidindo uma placa. Para esse caso, uma força de contato dissipativa contínua é escrita como:

$$
F\left(\delta_{n}\right)=\eta_{n, B} \delta_{n}^{3 / 2} \dot{\delta_{n}}
$$

Usando a Eq. (4.69), a equação do movimento da sobreposição normal é expressa por:

$$
m_{e f f} \ddot{\delta}_{n}+\eta_{n, B} \delta_{n}^{3 / 2} \dot{\delta}_{n}+k_{n, H z} \delta_{n}^{3 / 2}=0
$$

onde $\eta_{n, B}$ é o coeficiente de amortecimento não-linear para esse modelo contínuo. Usando na Eq. (4.70) os parâmetros adimensionais da Eq. (4.52), pode-se escrever a equação de deslocamento para a sobreposição normal como:

$$
\delta_{n}^{* \prime \prime}+2 \nu_{B}^{*} \delta_{n}^{* 3 / 2} \delta_{n}^{* \prime}+\delta_{n}^{* 3 / 2}=0
$$

onde como anteriormente as aspas indicam derivadas em relação ao tempo adimensional $t^{*}$. O parâmetro $\nu_{B}^{*}$ é o coeficiente de amortecimento adimensional dado por:

$$
\nu_{B}^{*}=\frac{1}{2} \frac{\eta_{n, B} v_{0}}{k_{n, H z}}
$$


Observa-se, através da Eq. (4.72), que o coeficiente de restituição diminui com a velocidade de impacto. Resolvendo numericamente a equação da sobreposição adimensional, (4.71), com condições iniciais $\delta_{n}^{*}(0)=0$ e $\delta_{n}^{* \prime}(0)=1$, determina-se a sobreposição máxima adimensional, a duração do contato adimensional e a sobreposição média adimensional, através do uso, respectivamente, das (4.55), (4.56), (4.57), durante o período de contato. As Figuras 22, 23, 24 apresentam curvas para o modelo não-linear com potência no termo de amortecimento $3 / 2$, da sobreposição adimensional versus o tempo adimensional, da velocidade adimensional versus o tempo adimensional e da aceleração adimensional versus o tempo adimensional, para valores do coeficiente de amortecimento $\nu_{B}^{*}=0,0.1,0.25,0.4,0.6$. Nota-se na Fig. 24 que a força de contato é contínua entre o início e o final do impacto entre os corpos, isto é, a aceleração é igual ao valor zero nesses dois instantes.

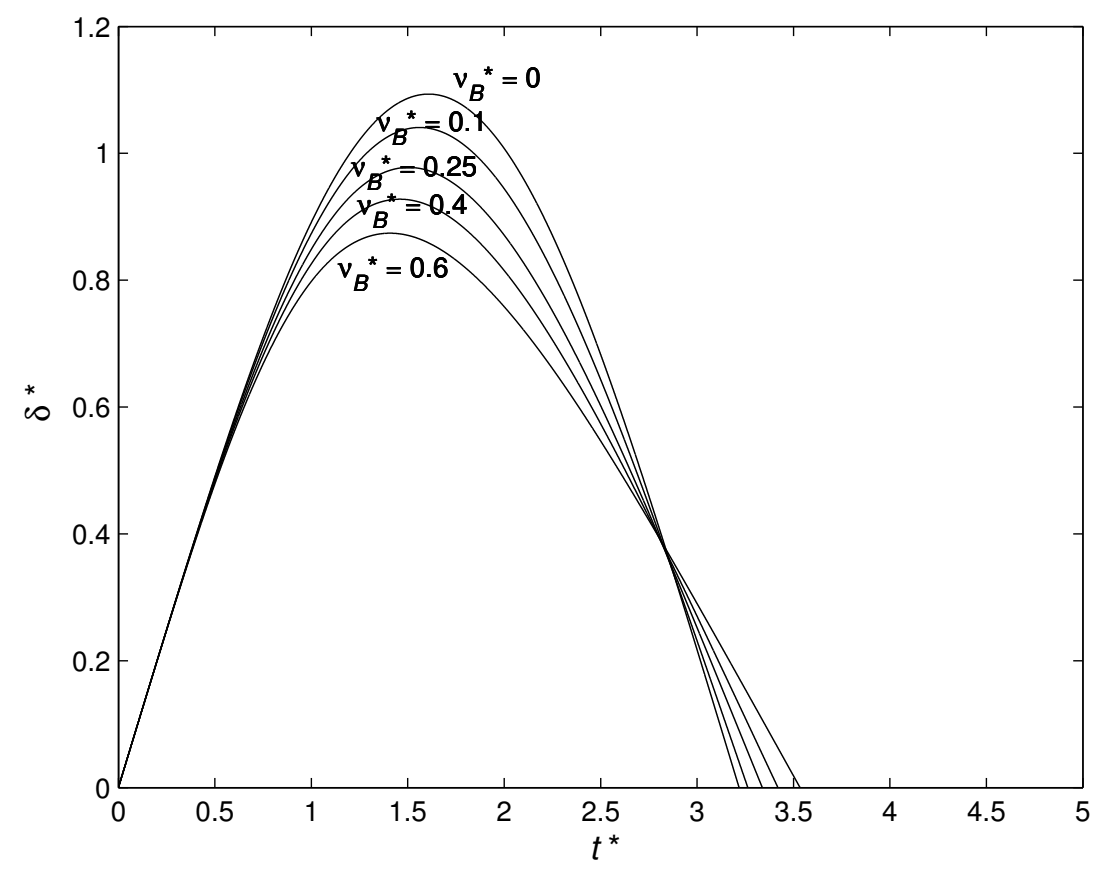

Figura 22 - Sobreposição adimensional (modelo não-linear, Eq. (4.71)).

A Tabela 11 mostra, para os diversos valores do coeficiente de amortecimento adimensional, $\left(\nu_{B}^{*}\right)$, os valores adimensionais dos seguintes parâmetros: coeficiente de restituição, $e_{n}$, instante da sobreposição máxima, $t_{\text {max }}^{*}$, sobreposição máxima, $\delta_{\text {max }}^{*}$, sobreposição média, $\bar{\delta}_{n}^{*}$, duração do contato, $t_{c, n}^{*}$, aceleração inicial, $a^{*}(0)$ e aceleração final, $a^{*}\left(t_{c, n}^{*}\right)$. Esses parâmetros são calculados numericamente por meio das Eqs. (4.55), (4.56) e (4.57). O coeficiente de restituição e aceleração são calculados, utilizando, respectivamente, as seguintes expressões: $e_{n}=-\delta_{n}^{* \prime}\left(t_{c, n}^{*}\right)$ e $a^{*}=v^{* \prime}$. Na Tabela 11 observa-se, também, que a aceleração é nula no início e no final do impacto produzindo uma força contínua nesses dois instantes.

Para determinar uma relação entre o coeficiente de amortecimento adimensional 


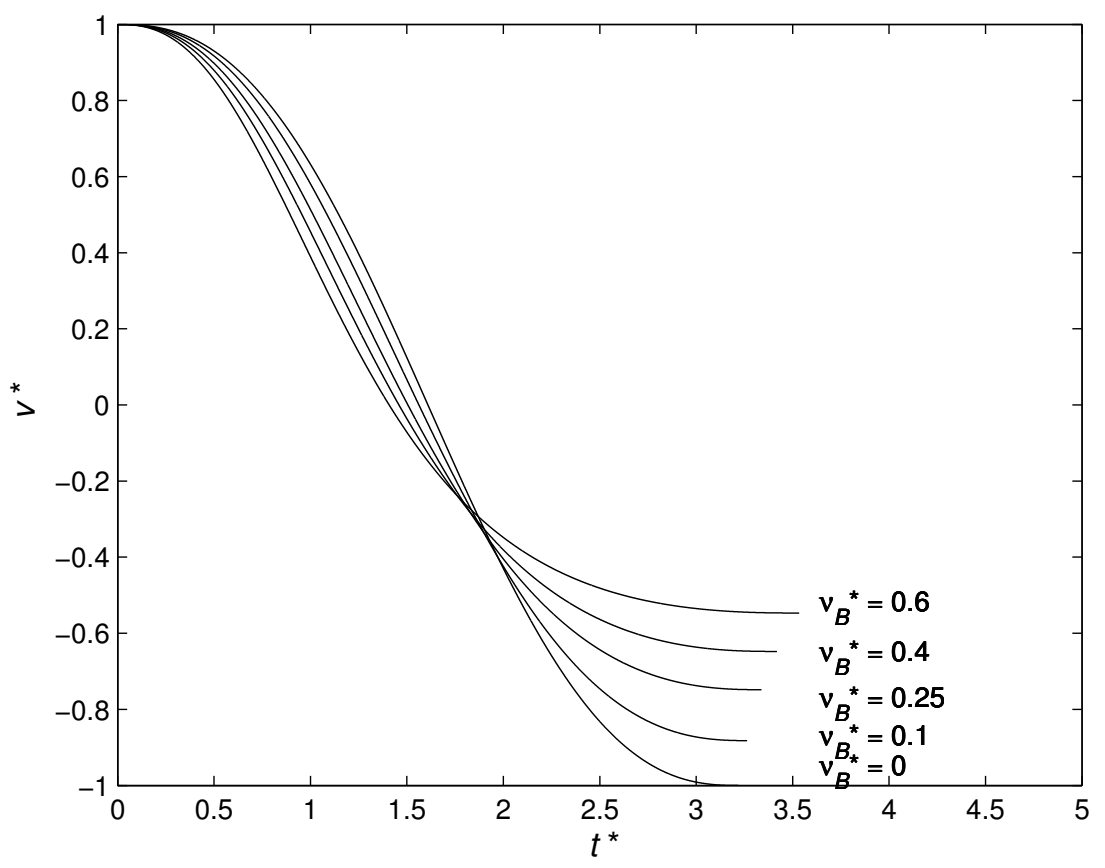

Figura 23 - Velocidade adimensional (modelo não-linear, Eq. (4.71)).

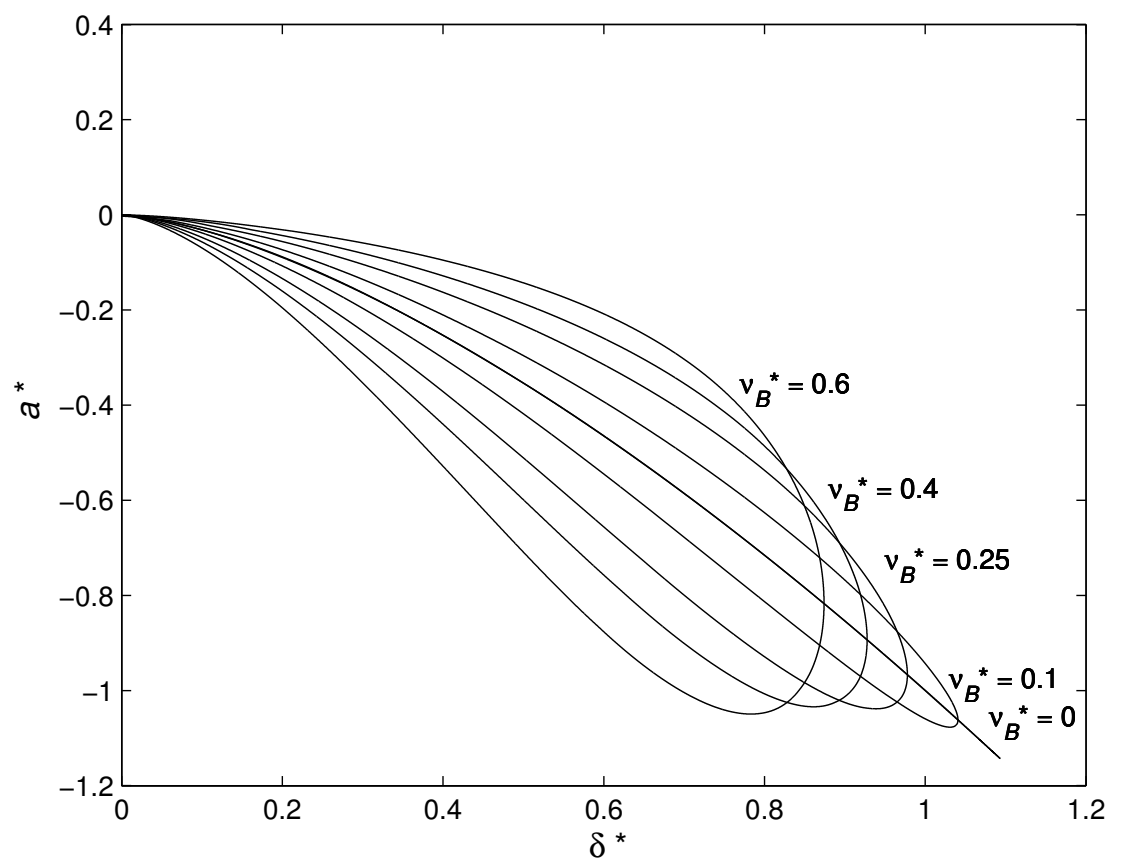

Figura 24 - Aceleração adimensional (modelo não-linear, Eq. (4.71)). 
Tabela 11 - Parâmetros adimensionais para o modelo não-linear, Eq. (4.71).

\begin{tabular}{llllllll}
\hline$\nu_{B}^{*}$ & $e_{n}$ & $t_{\max }^{*}$ & $\delta_{\max }^{*}$ & $\bar{\delta}_{n}^{*}$ & $t_{c, n}^{*}$ & $a^{*}(0)$ & $a^{*}\left(t_{c, n}^{*}\right)$ \\
\hline 0 & 1.0000 & 1.6090 & 1.0934 & 0.6833 & 3.2181 & 0 & 0 \\
0.02 & 0.9740 & 1.5990 & 1.0819 & 0.6762 & 3.2268 & 0 & 0 \\
0.04 & 0.9494 & 1.5893 & 1.0710 & 0.6693 & 3.2356 & 0 & 0 \\
0.06 & 0.9259 & 1.5799 & 1.0605 & 0.6627 & 3.2447 & 0 & 0 \\
0.08 & 0.9035 & 1.5709 & 1.0504 & 0.6563 & 3.2539 & 0 & 0 \\
0.1 & 0.8822 & 1.5622 & 1.0407 & 0.6501 & 3.2633 & 0 & 0 \\
0.15 & 0.8329 & 1.5417 & 1.0179 & 0.6355 & 3.2875 & 0 & 0 \\
0.2 & 0.7885 & 1.5228 & 0.9970 & 0.6220 & 3.3125 & 0 & 0 \\
0.25 & 0.7484 & 1.5052 & 0.9778 & 0.6094 & 3.3382 & 0 & 0 \\
0.3 & 0.7120 & 1.4888 & 0.9599 & 0.5977 & 3.3646 & 0 & 0 \\
0.35 & 0.6786 & 1.4734 & 0.9433 & 0.5867 & 3.3916 & 0 & 0 \\
0.4 & 0.6480 & 1.4589 & 0.9277 & 0.5763 & 3.4192 & 0 & 0 \\
0.45 & 0.6197 & 1.4452 & 0.9131 & 0.5665 & 3.4472 & 0 & 0 \\
0.5 & 0.5936 & 1.4323 & 0.8994 & 0.5573 & 3.4757 & 0 & 0 \\
0.55 & 0.5694 & 1.4200 & 0.8864 & 0.5485 & 3.5046 & 0 & 0 \\
0.6 & 0.5469 & 1.4083 & 0.8742 & 0.5401 & 3.5339 & 0 & 0 \\
0.7 & 0.5062 & 1.3865 & 0.8515 & 0.5245 & 3.5936 & 0 & 0 \\
0.8 & 0.4704 & 1.3666 & 0.8309 & 0.5102 & 3.6547 & 0 & 0 \\
0.9 & 0.4388 & 1.3482 & 0.8122 & 0.4970 & 3.7169 & 0 & 0 \\
1.0 & 0.4107 & 1.3311 & 0.7949 & 0.4848 & 3.7803 & 0 & 0 \\
1.05 & 0.3978 & 1.3230 & 0.7868 & 0.4790 & 3.8123 & 0 & 0 \\
\hline
\end{tabular}

não-linear , $\nu_{B}^{*}$, em função do coeficiente de restituição, utiliza-se o mesmo procedimento de Antypov e Elliott (2011). Substituindo as relações (4.59) na Eq. (4.71) obtém-se $n=2 / 5$ e $A=(5 / 2)^{2 / 5}$. Assumindo que as velocidades permanecem inalteradas, conforme:

$$
v^{*}\left(t^{*}\right)=\hat{v}\left(\tau_{2}\right) \quad \hat{v}\left(\tau_{2}\right)=\frac{d x}{d \tau_{2}}=x^{\prime},
$$

tem-se:

$$
x^{\prime \prime}+2 \nu_{B}^{*} x^{\prime}+1=0 .
$$

A sobreposição é reescalonada pela Eq. (4.61) da forma:

$$
\delta_{n}^{*}=(5 / 2 x)^{2 / 5} .
$$

Como não é possível relacionar a Eq. (4.74) com o modelo linear, resolve-se a Eq. (4.74), com condições iniciais $x(0)=0$ e $x^{\prime}(0)=1$ obtendo-se:

$$
\begin{gathered}
x\left(\tau_{2}\right)=\frac{1}{4 \nu_{B}^{* 2}}\left(2 \nu_{B}^{*}+1-2 \nu_{B}^{*} \tau_{2}-\left(2 \nu_{B}^{*}+1\right) \exp \left(-2 \nu_{B}^{*} \tau_{2}\right)\right) \quad \text { se } \quad \nu_{B}^{*}>0 \\
x\left(\tau_{2}\right)=\left(1-\frac{\tau_{2}}{2}\right) \tau_{2} \quad \text { se } \quad \nu_{B}^{*}=0 \\
\text { e como } e_{n}=-\delta_{n}^{* \prime}\left(t_{c, n}^{*}\right)=-v^{*}\left(t_{c, n}^{*}\right)=-x^{\prime}\left(\tau_{c, 2}\right) \text { tem-se: } \\
e_{n}=\frac{1}{2 \nu_{B}^{*}}\left(1-\left(2 \nu_{B}^{*}+1\right) \exp \left(-2 \nu_{B}^{*} \tau_{2, c}\right)\right) .
\end{gathered}
$$


No final do contato a sobreposição é nula $x\left(\tau_{2, c}\right)=0$, e de acordo com a Eq. (4.76) a duração do contato reescalonada, $\tau_{2, c}$, é calculada por:

$$
\begin{array}{r}
\left(2 \nu_{B}^{*}+1\right) \exp \left(-2 \nu_{B}^{*} \tau_{2, c}\right)+2 \nu_{B}^{*} \tau_{2, c}-\left(2 \nu_{B}^{*}+1\right)=0 \quad \text { se } \quad \nu_{B}^{*}>0 \\
\tau_{2, c}=2 \quad \text { se } \quad \nu_{B}^{*}=0
\end{array}
$$

e relacionando a Eq. (4.77) com a Eq. (4.78) o coeficiente de restituição é representado por $e_{n}=\tau_{2, c}-1$. Observa-se nas Eqs. (4.77) e (4.78) que não é possível encontrar uma relação explícita para $\nu_{B}^{*}$ em função de $e_{n}$. Na literatura existem diversas relações aproximadas para o cálculo do coeficiente de amortecimento $\eta_{n, B}$ ou $\nu_{B}^{*}$. Hunt e Grossley (1975) propuseram a seguinte relação para o cálculo do coeficiente de amortecimento $\eta_{n, B}$ :

$$
\eta_{n, B}=\frac{3}{2} \frac{k_{n, H z}\left(1-e_{n}\right)}{v_{0}}
$$

Substituindo a Eq.(4.79) na Eq. (4.72), o coeficiente de amortecimento adimensional é dado por:

$$
\nu_{B}^{*}=\frac{3}{4}\left(1-e_{n}\right)
$$

Utilizando a análise feita por Hunt e Grossley (1975), Lankarani e Nikravesh (1990) e Lankarani e Nikravesh (1994) propuseram um modelo para $\eta_{n, B}$ através da seguinte relação:

$$
\eta_{n, B}=\frac{3}{4} \frac{k_{n, H z}\left(1-e_{n}^{2}\right)}{v_{0}}
$$

Como anteriormente, utilizando as Eqs. (4.72) e (4.81), o coeficiente de amortecimento adimensional é calculado por:

$$
\nu_{B}^{*}=\frac{3}{8}\left(1-e_{n}^{2}\right)
$$

Os modelos propostos por Hunt e Grossley (1975) e Lankarani e Nikravesh (1990), são válido para altos valores do coeficiente de restituição, como o impacto entre metais. Flores et al. (2011) avaliaram a dissipação de energia do amortecimento modelando o processo de contato como um sistema de um grau de liberdade obtendo as seguintes expressões para $\eta_{n, B}$ e $\nu_{B}^{*}$ :

$$
\begin{gathered}
\eta_{n, B}=\frac{8}{5} \frac{k_{n, H z}\left(1-e_{n}\right)}{e_{n} v_{0}} \\
\nu_{B}^{*}=\frac{4}{5} \frac{\left(1-e_{n}\right)}{e_{n}}
\end{gathered}
$$


Hu et al. (2011) propuseram uma expressão para o coeficiente de amortecimento adimensional obtida através de ajuste não-linear realizado numericamente. A relação obtida pelos autores é válida para $\left.e_{n} \in[0.05,1.0]\right)$ e representada por:

$$
\nu_{B}^{*}=-\frac{1}{2} \ln e_{n} \frac{6.66264}{3.85238+\ln e_{n}}
$$

Jordam-Caserta (2015) derivou uma relação aproximada para o calculo de $\nu_{B}^{*}$. A autora baseou no trabalho de $\mathrm{Hu}$ et al. (2011) acrescentando um novo parâmetro multiplicando o termo logaritmo do denominador da Eq. (4.85). Jordam-Caserta (2015), também, utilizou ajuste não linear, entretanto a faixa de valores do coeficiente de restituição é um pouco mais restrita, isto é, $e_{n} \in[0.3978,1.0],\left(\nu_{B}^{*} \in[0,1.05]\right)$. Entretanto, observa-se que nessa faixa de valores ocorrem diversos casos práticos de tipos de contato. A expressão obtida por Jordam-Caserta (2015) é a seguinte (ver também (JORDAM-CASERTA; NAVARRO; CABEZAS-GóMEZ, 2016)):

$$
\nu_{B}^{*}=-\frac{1}{2} \ln e_{n} \frac{8.693764}{5.573076+1.966760 \ln e_{n}}
$$

A Tabela 12 exibe uma comparação com os valores de $\nu_{B}^{*}$ obtidos pelas expressões (4.80), (4.82), (4.84), (4.85) e (4.86) com os valores de $e_{n}$ e $\nu_{B}^{*}$ calculados numericamente de acordo com a Tabela 11 . Observa-se que para altos valores de $e_{n}\left(e_{n}>\approx 0.94\right)$, os modelos (Eqs. 4.80, 4.82, 4.84, e 4.86) se aproximam dos valores de $\nu_{B}^{*}$ (ver segunda coluna da Tabela 12). O modelo proposto por Flores et al. (2011), Eq.(4.84), aproxima da solução numérica para valores do coeficiente de restituição no seguinte intervalo aproximado: $\left(\approx 0.64<e_{n} \leq 1.0\right)$. Os valores obtidos por ajuste numérico pelas Eqs. (4.85 e 4.86) estão próximos dos valores numéricos de $\nu_{B}^{*}$ mostrados na Tabela 12 para faixas específicas de $e_{n}$. Para o intervalo $e_{n} \in[0.3978,1.0],\left(\nu_{B}^{*} \in[0,1.05]\right)$, o erro relativo médio para a Eq. (4.86) é de $0.799 \%$ e para a Eq. (4.85) é de $5.105 \%$. Dessa forma é recomendado o uso da Eq. (4.86) no intervalo $e_{n} \in[0.3978,1.0]$ e a Eq. (4.85) fora desse intervalo, isto é, para o valor do coeficiente de restituição $e_{n} \in[0.05,0.4]$.

Diversos trabalhos apresentam modelos para o cálculo da duração de contato durante a colisão, tais como: Lankarani e Nikravesh (1994), Antypov e Elliott (2011), Navarro e Braun (2013), Kruggel-Emden et al. (2007), entre outros. Utilizando a Tabela 11 deriva-se uma nova relação para o cálculo do tempo de contato adimensional, $t_{c, n}^{*}$, por meio de ajuste não-linear por mínimos quadrados para a faixa do coeficiente de amortecimento adimensional $\nu_{B}^{*} \in[0,1.05]\left(\Delta_{\nu_{B}^{*}}=0.01\right)$. A relação para $t_{c, n}^{*}$ em função do coeficiente de restituição $e_{n} \in[0.3978,1.0]$ é expressa por:

$$
t_{c, n}^{*}=3.21806\left(1-0.425661\left|\ln e_{n}\right|^{1.164355}\right)^{-0.347025}
$$


Tabela 12 - Comparação dos valores de $\nu_{B}^{*}$ para diversos modelos da força dissipativa.

\begin{tabular}{lllllll}
\hline$e_{n}$ & $\nu_{B}^{*}$ & $\nu_{B}^{*}$ (Eq.4.80) & $\nu_{B}^{*}$ (Eq.4.82) & $\nu_{B}^{*}$ (Eq.4.84) & $\nu_{B}^{*}$ (Eq.4.85) & $\nu_{B}^{*}$ (Eq.4.86) \\
\hline 1.0000 & 0 & 0 & 0 & 0 & 0 & 0 \\
0.9740 & 0.02 & 0.0195 & 0.0192 & 0.0214 & 0.0229 & 0.0207 \\
0.9494 & 0.04 & 0.0379 & 0.0370 & 0.0426 & 0.0455 & 0.0413 \\
0.9259 & 0.06 & 0.0556 & 0.0535 & 0.0640 & 0.0679 & 0.0617 \\
0.9035 & 0.08 & 0.0724 & 0.0689 & 0.0854 & 0.0901 & 0.0821 \\
0.8822 & 0.1 & 0.0884 & 0.0831 & 0.1068 & 0.1120 & 0.1023 \\
0.8329 & 0.15 & 0.1253 & 0.1149 & 0.1605 & 0.1660 & 0.1525 \\
0.7885 & 0.2 & 0.1586 & 0.1419 & 0.2146 & 0.2190 & 0.2023 \\
0.7484 & 0.25 & 0.1887 & 0.1650 & 0.2689 & 0.2710 & 0.2518 \\
0.7120 & 0.3 & 0.2160 & 0.1849 & 0.3236 & 0.3221 & 0.3011 \\
0.6786 & 0.35 & 0.2411 & 0.2023 & 0.3789 & 0.3728 & 0.3504 \\
0.6480 & 0.4 & 0.2640 & 0.2175 & 0.4346 & 0.4228 & 0.3996 \\
0.6197 & 0.45 & 0.2852 & 0.2310 & 0.4909 & 0.4725 & 0.4490 \\
0.5936 & 0.5 & 0.3048 & 0.2429 & 0.5477 & 0.5216 & 0.4985 \\
0.5694 & 0.55 & 0.3229 & 0.2534 & 0.6050 & 0.5704 & 0.5482 \\
0.5469 & 0.6 & 0.3398 & 0.2628 & 0.6628 & 0.6188 & 0.5982 \\
0.5062 & 0.7 & 0.3704 & 0.2789 & 0.7804 & 0.7151 & 0.6991 \\
0.4704 & 0.8 & 0.3972 & 0.2920 & 0.9007 & 0.8109 & 0.8014 \\
0.4388 & 0.9 & 0.4209 & 0.3028 & 1.0232 & 0.9060 & 0.9056 \\
0.4107 & 1.0 & 0.4420 & 0.3117 & 1.1478 & 1.0006 & 1.0118 \\
0.3978 & 1.05 & 0.4517 & 0.3157 & 1.2111 & 1.0479 & 1.0657 \\
\hline
\end{tabular}

A Tabela 13 apresenta uma comparação da duração de contato para o modelo não-linear (Eq. 4.71) através dos valores obtidos da Tabela 11 e da Eq. (4.87). O erro relativo médio obtido foi de $0.0236 \%$. Desta forma a relação, Eq. (4.87), representa uma boa aproximação para o cálculo de $t_{c, n}^{*}$.

As forças tangenciais elásticas nos modelos não-lineares A (4.53) e B (4.71) são calculadas usando a Eq. (4.37). Portanto, a força elástica total nesses modelos é dada por:

$$
\mathbf{F}_{i j}^{S}=-\left[k_{n, H z} \delta_{n}{ }^{3 / 2} \mathbf{n}_{i j}+k_{t, H z} \delta_{n}{ }^{1 / 2} \delta_{\mathbf{t}}\right]
$$

O cálculo das forças tangencias dissipativas relacionam com a rigidez da mola tangencial e com o coeficiente de restituição tangencial por meio das Eqs. (4.54) e (4.58) no modelo A e das Eqs. (4.72) e (4.86) no modelo B. Portanto, as forças dissipativas totais são expressas nos modelos A e B, respectivamente, por:

$$
\begin{gathered}
\mathbf{F}_{i j}^{D}=-\sqrt{5} \delta_{n}{ }^{1 / 4}\left[\sqrt{\left(m_{e f f} k_{n, H z}\right)} \frac{\left|\ln e_{n}\right|}{\sqrt{\ln ^{2} e_{n}+\pi^{2}}} \mathbf{V}_{n, i j}+\sqrt{\left(m_{e f f} k_{t, H z}\right)} \frac{\left|\ln e_{t}\right|}{\sqrt{\ln ^{2} e_{t}+\pi^{2}}} \mathbf{V}_{t, i j}\right] \\
\mathbf{F}_{i j}^{D}=-\frac{\delta_{n}^{3 / 2}}{v_{0}}\left[k_{n, H z} \frac{8.693764\left|\ln e_{n}\right|}{5.573076+1.966760 \ln e_{n}} \mathbf{V}_{n, i j}+k_{t, H z} \frac{8.693764\left|\ln e_{t}\right|}{5.573076+1.966760 \ln e_{t}} \mathbf{V}_{t, i j}\right]
\end{gathered}
$$


Tabela 13 - Duração da colisão, $t_{c, n}^{*}$, para o modelo não-linear , Eq. (4.71).

\begin{tabular}{llll}
\hline$\nu_{B}^{*}$ & $e_{n}$ & $t_{c, n}^{*}$, Tabela 11 & $t_{c, n}^{*}$, Eq. $(4.87)$ \\
\hline 0 & 1.0000 & 3.2181 & 3.2181 \\
0.02 & 0.9740 & 3.2268 & 3.2250 \\
0.04 & 0.9494 & 3.2356 & 3.2334 \\
0.06 & 0.9259 & 3.2447 & 3.2424 \\
0.08 & 0.9035 & 3.2539 & 3.2518 \\
0.1 & 0.8822 & 3.2633 & 3.2615 \\
0.15 & 0.8329 & 3.2875 & 3.2865 \\
0.2 & 0.7885 & 3.3125 & 3.3123 \\
0.25 & 0.7484 & 3.3382 & 3.3387 \\
0.3 & 0.7120 & 3.3646 & 3.3655 \\
0.35 & 0.6786 & 3.3916 & 3.3927 \\
0.4 & 0.6480 & 3.4192 & 3.4202 \\
0.45 & 0.6197 & 3.4472 & 3.4481 \\
0.5 & 0.5936 & 3.4757 & 3.4763 \\
0.55 & 0.5694 & 3.5046 & 3.5049 \\
0.6 & 0.5469 & 3.5339 & 3.5339 \\
0.7 & 0.5062 & 3.5936 & 3.5930 \\
0.8 & 0.4704 & 3.6547 & 3.6538 \\
0.9 & 0.4388 & 3.7169 & 3.7164 \\
1.0 & 0.4107 & 3.7803 & 3.7810 \\
1.05 & 0.3978 & 3.8123 & 3.8141 \\
\hline
\end{tabular}

A seguir são comparados os dois modelos não-lineares de esfera-suave (A - Eq.(4.53)) e B - Eq. (4.71))) discutidos no presente trabalho. Analisando a duração do contato, $t_{c, n}^{*}$, com o coeficiente de restituição na direção normal, $e_{n}$, para quatro valores do coeficiente de amortecimento $\left(\nu_{A, B}^{*}=[0.02,0.04,0.1,0.3]\right)$ observa-se que a duração de contato é menor $(0.30 \%, 0.63 \%, 1.75 \%, 7.17 \%)$ em todos os casos para o modelo B. O modelo não-linear B também resulta em colisões mais elásticas para as partículas, pois os valores dos coeficientes de restituição obtidos são maiores dos que os do modelo A $(3.04 \%, 6.23 \%, 17.0 \%, 70.8 \%)$. A aceleração no final da colisão assume valores nulos ou próximos de zero para ambos os modelos, entretanto o modelo A tem características atrativas durante o final do contato (ver Figura 21).

A seguir são comparados os modelos não-lineares A e B com as medidas experimentais do coeficiente de restituição e da duração da colisão. Para esse propósito utiliza-se o procedimento descrito por Stevens e Hrenya (2005) e os dados experimentais que os autores obtiveram para colisão binária entre esferas de aço inoxidável (STEVENS; HRENYA, 2005). As propriedades dos materiais são: módulo de Young de $193 \mathrm{GPa}$; coeficiente de Poisson de 0.35 ; densidade de $8030 \mathrm{~kg} / \mathrm{m}^{3}$; e raio de $1.27 \mathrm{~cm}$. A faixa das velocidades de impacto é de $V_{i m p}=0.2 \mathrm{~m} / \mathrm{s}-1.5 \mathrm{~m} / \mathrm{s}$. Seguindo o trabalho de Stevens e Hrenya (2005), é escolhido um parâmetro de entrada de tal modo que as predições do modelo equiparem aos dos dados experimentais do coeficiente de restituição $(e=0.879)$ na velocidade intermediária 
de impacto de $V_{i m p}=0.84 \mathrm{~m} / \mathrm{s}$.

A Figura 25 mostra as predições dos modelos (A e B) e os dados experimentais para o coeficiente de restituição em função da velocidade de impacto. Observa-se que o modelo $\mathrm{B}$ e os dados experimentais apresentam uma tendência de decaimento, isto é o coeficiente de restituição diminui com a velocidade de impacto. No modelo A (linha tracejada na Figura 25), o coeficiente de restituição é independente da velocidade de impacto. Esse comportamento é inconsistente com os dados reais. A Figura 26 ilustra a variação da duração da colisão com a velocidade de impacto. Ambos modelos (A e B) apresentam resultados precisos em comparação com os dados experimentais. Nesse gráfico nota-se também que os dois modelos de esfera-suave são consistentes com os dados experimentais, isto é, a duração do contato diminui com o aumento da velocidade de impacto.

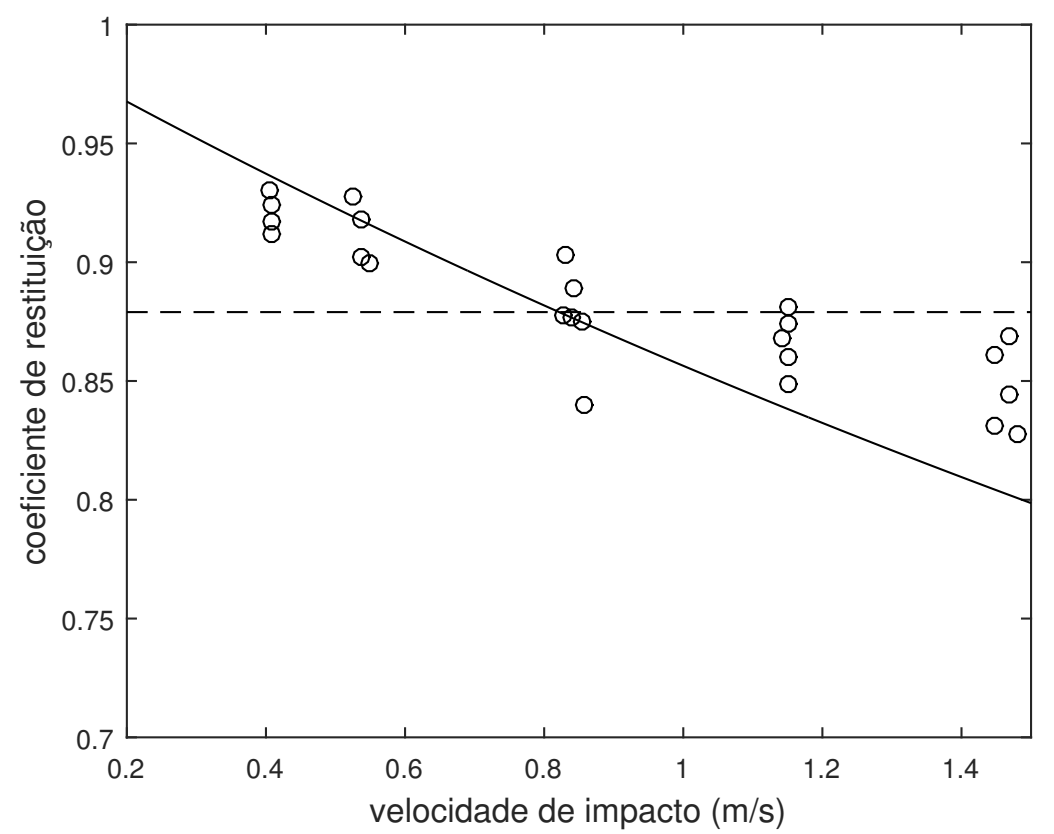

Figura 25 - Comparação dos dados experimentias (circulos (STEVENS; HRENYA, 2005)) com os modelos de esfera suave para o coeficiente de restituição (modelo A: linha tracejada; modelo B: linha contínua).

Com essas características físicas consistente do modelo B, inclusive com a duração do contato menor, esse modelo resultará em tempos computacionais menores o que é fundamental para a simulação de sistemas complexos, como de escoamentos gás-sólido e de granulares.

\subsection{Coeficiente de rigidez - modelo linear}

Nesta seção é descrito um método para determinar o coeficiente de rigidez para o modelo linear, $k_{n}$, tomando como equivalente os modelos não-lineares descritos anteriormente. Essa analogia é feita de três maneiras diferentes, isto é, através da sobreposição 


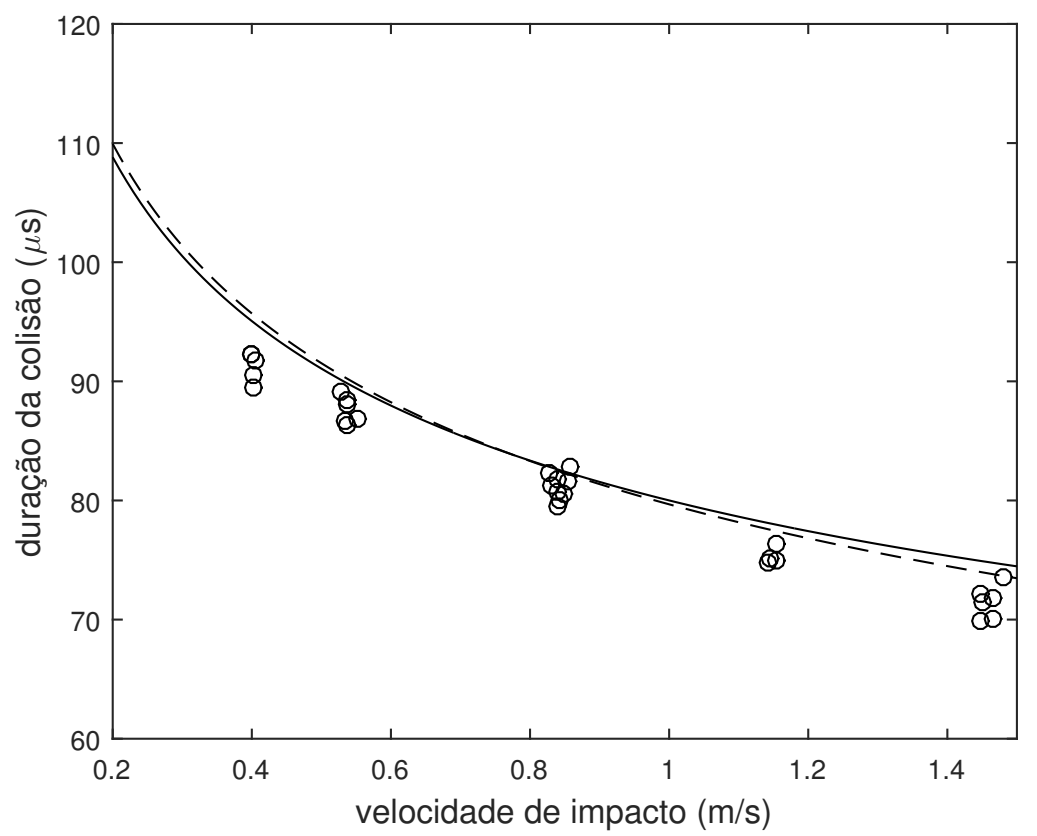

Figura 26 - Comparação dos dados experimentias (circulos (STEVENS; HRENYA, 2005)) com os modelos de esfera suave para a duração da colisão (modelo A: linha tracejada; modelo B: linha contínua).

máxima, da duração do contato e da sobreposição média. Considerando a sobreposição máxima, para $e_{n}<1.0$, a equivalência é dada por:

$$
\delta_{\max L}=\delta_{\max N L}
$$

Substituindo a sobreposição máxima do modelo linear $\left(\delta_{\max L}\right)$ pela Eq. $(4.27)$

$$
\left(v_{0} / \Omega_{0}\right) \exp \left(-\frac{\arctan (\beta)}{\beta}\right)=\delta_{\max N L}
$$

Substituindo a sobreposição máxima do modelo não-linear $\left(\delta_{\max N L}\right)$ pelo valor adimensional $\delta_{\operatorname{maxNL}}^{*}=\delta_{\max N L}\left(\frac{k_{n, H z}}{v_{0}^{2} m_{e f f}}\right)^{2 / 5}$ (Eq. 4.52):

$$
\left(v_{0} / \Omega_{0}\right) \exp \left(-\frac{\arctan (\beta)}{\beta}\right)=\delta_{\operatorname{maxNL}}^{*}\left(\frac{k_{n, H z}}{v_{0}^{2} m_{e f f}}\right)^{-2 / 5}
$$

Isolando $\Omega_{0}$

$$
\Omega_{0}=\frac{v_{0}}{\delta_{\max H S D-A, B}^{*}} \exp \left(-\frac{\arctan (\beta)}{\beta}\right)\left(\frac{k_{n, H z}}{v_{0}^{2} m_{\text {eff }}}\right)^{2 / 5}
$$

Substituindo $\Omega_{0}$ por $\Omega_{0}=\sqrt{k_{n} / m_{\text {eff }}}$

$$
\sqrt{k_{n} / m_{\text {eff }}}=\frac{v_{0}}{\delta_{\max N L}^{*}} \exp \left(-\frac{\arctan (\beta)}{\beta}\right)\left(\frac{k_{n, H z}}{v_{0}^{2} m_{e f f}}\right)^{2 / 5}
$$

Isolando $k_{n}$, obtém-se

$$
k_{n}=\frac{1}{\delta_{\max N L}^{* 2}}\left(v_{0}^{1 / 2} m_{e f f}^{1 / 4} k_{n, H z}\right)^{4 / 5}\left(\exp \left(-\frac{\arctan (\beta)}{\beta}\right)\right)^{2}
$$


Considerando a equivalência entre a duração do contato linear e a duração de contato não-linear, tem-se, para $e_{n}<1.0$ :

$$
t_{c, n L}=t_{c, n N L}
$$

Substituindo a duração do contato linear $\left(t_{c, n L}\right)$ pela Eq. (4.28)

$$
\frac{\pi}{\Omega_{0}} \sqrt{\left(1+\frac{1}{\beta^{2}}\right)}=t_{c, n N L}
$$

Substituindo a duração do contato não-linear $\left(t_{c, n N L}\right)$ pelo seguinte valor adimensional $t_{c, n N L}^{*}=t_{c, n N L}\left(\frac{k_{n, H z} v_{0}^{1 / 2}}{m_{e f f}}\right)^{2 / 5}($ Eq. 4.52$)$ :

$$
\frac{\pi}{\Omega_{0}} \sqrt{\left(1+\frac{1}{\beta^{2}}\right)}=t_{c, n N L}^{*}\left(\frac{k_{n, H z} v_{0}^{1 / 2}}{m_{e f f}}\right)^{-2 / 5}
$$

Isolando $\Omega_{0}$

$$
\Omega_{0}=\frac{\pi}{t_{c, n N L}^{*}} \sqrt{\left(1+\frac{1}{\beta^{2}}\right)}\left(\frac{k_{n, H z} v_{0}^{1 / 2}}{m_{e f f}}\right)^{2 / 5}
$$

Substituindo $\Omega_{0}=\sqrt{k_{n} / m_{\text {eff }}}$ e isolando $k_{n}$ tem-se que:

$$
k_{n}=\frac{1}{t_{c, n N L}^{* 2}}\left(v_{0}^{1 / 2} m_{e f f}^{1 / 4} k_{n, H z}\right)^{4 / 5} \pi^{2}\left(1+\frac{1}{\beta^{2}}\right)
$$

De modo similar, para a sobreposição média a equivalência para $e_{n}<1.0$ é dada por:

$$
\bar{\delta}_{L}=\bar{\delta}_{N L}
$$

Substituindo a sobreposição média linear $\left(\bar{\delta}_{L}\right)$ pela Eq. (4.29)

$$
\frac{v_{0}}{\pi \Omega_{0}} \frac{-\beta}{\sqrt{1+\beta^{2}}}\left(1+\exp \left(\frac{\pi}{\beta}\right)\right)=\bar{\delta}_{N L}
$$

Substituindo a sobreposição média adimensional $\bar{\delta}_{\max L L}^{*}=\bar{\delta}_{N L}\left(\frac{k_{n, H z}}{v_{0}^{2} m_{\text {eff }}}\right)^{2 / 5}$ (Eq. 4.52), isolando $\Omega_{0}$, substituindo $\Omega_{0}=\sqrt{k_{n} / m_{\text {eff }}}$ e isolando $k_{n}$, obtém-se:

$$
k_{n}=\frac{1}{\bar{\delta}_{N L}^{* 2}}\left(v_{0}^{1 / 2} m_{e f f}^{1 / 4} k_{n, H z}\right)^{4 / 5} \frac{\beta^{2}}{\pi^{2}\left(1+\beta^{2}\right)}\left(1+\exp \left(\frac{\pi}{\beta}\right)\right)^{2}
$$

onde $\delta_{\max L L}^{*}, t_{c, N L}^{*}$, e $\bar{\delta}_{N L}^{*}$ são parâmetros adimensionais calculados para os modelos nãolineares pelos algoritmos numéricos dados pelas Eqs. (4.55), (4.56) e (4.57), respectivamente.

Para as colisões perfeitamente elástica $\left(e_{n}=1.0\right)$, a sobreposição máxima, a duração do contato e a sobreposição média são expressas pelas Eqs. (4.105), (4.106) e (4.107), respectivamente:

$$
k_{n}=\frac{1}{\delta_{\operatorname{maxNL}}^{* 2}}\left(v_{0}^{1 / 2} m_{e f f}^{1 / 4} k_{n, H z}\right)^{4 / 5}
$$




$$
\begin{aligned}
& k_{n}=\frac{\pi^{2}}{t_{c, n N L}^{* 2}}\left(v_{0}^{1 / 2} m_{e f f}^{1 / 4} k_{n, H z}\right)^{4 / 5} \\
& k_{n}=\frac{4}{\pi^{2} \bar{\delta}_{N L}^{* 2}}\left(v_{0}^{1 / 2} m_{e f f}^{1 / 4} k_{n, H z}\right)^{4 / 5}
\end{aligned}
$$

Foerster et al. (1994) realizaram um experimento para medir as propriedades de colisão entre duas pequenas esferas e entre uma pequenas esfera e uma superfície plana. Utilizando os dados experimentais do trabalho de Foerster et al. (1994) é determinado o coeficiente de rigidez para o modelo linear. Considera-se as esferas como partículas de vidro de diâmetro $3.18 \mathrm{~mm}$ colidindo a uma velocidade de $v_{0}=1 \mathrm{~m} / \mathrm{s}$. As propriedades das partículas de vidro são: massa específica $\rho=2500 \mathrm{~kg} / \mathrm{m}^{3}$, módulo de Young $E=$ $71 \mathrm{GPa}$, coeficiente de Poisson $\sigma=0.22$ e coeficiente de restituição normal $e_{n}=0.97$ (ver (FOERSTER et al., 1994)). Utilizando esses valores são calculados: massa efetiva $m_{\text {eff }}=2.105 \times 10^{-5} \mathrm{~kg}$, raio efetivo $r_{\text {eff }}=7.950 \times 10^{-4} \mathrm{~m}$, módulo de Young efetivo $E_{e f f}=37.306 \mathrm{GPa}$, coeficiente de rigidez não-linear $k_{n, H z}=1.4025 \times 10^{9} \mathrm{~N} / \mathrm{m}^{1.5}$. A Tabela 14 mostra os valores determinados para o coeficiente de rigidez linear para uma colisão binária entre duas esferas.

\begin{tabular}{|c|c|c|c|c|}
\hline Modelo & $\nu_{A, B}^{*}$ & $\begin{array}{l}\delta_{\max N L-A, B}^{*} \\
k_{n}[\mathrm{MN} / \mathrm{m}](\text { Eq.4.96) }\end{array}$ & $\begin{array}{l}t_{c, n N L-A, B}^{*} \\
k_{n}[\mathrm{MN} / \mathrm{m}](\text { Eq.4.101) }\end{array}$ & $\begin{array}{l}\bar{\delta}_{n N L-A, B}^{*} \\
k_{n}[\mathrm{MN} / \mathrm{m}] \text { (Eq.4.104) }\end{array}$ \\
\hline $\mathrm{A}$ & 0.0108 (Eq.4.58) & 1.0802 & 3.2280 & 0.6751 \\
\hline & & 2.0045 & 2.2836 & 2.0804 \\
\hline B & 0.0265 (Eq.4.85) & 1.0783 & 3.2296 & 0.6739 \\
\hline & & 2.0115 & 2.2813 & 2.0877 \\
\hline B & 0.0240 (Eq.4.86) & $\begin{array}{l}1.0797 \\
2.0063\end{array}$ & $\begin{array}{l}3.2285 \\
2.2829\end{array}$ & $\begin{array}{l}0.6748 \\
2.0822\end{array}$ \\
\hline
\end{tabular}

Tabela 14 - Coeficiente de rigidez normal para o modelo linear: colisão binária.

A seguir é determinado o coeficiente de rigidez linear para uma colisão entre uma esfera de vidro e uma superfície plana de alumínio. A velocidade de impacto é $v_{0}=1.35 \mathrm{~m} / \mathrm{s}$. Como anteriormente os dados experimentais são obtidos de Foerster et al. (1994). Para a superfície de alumínio são utilizadas as seguintes propriedades (FOERSTER et al., 1994): massa específica $\rho_{w}=2700 \mathrm{~kg} / \mathrm{m}^{3}$, módulo de Young $E_{w}=69 \mathrm{GPa}$, coeficiente de Poisson $\sigma_{w}=0.33$ e coeficiente de restituição normal para a colisão com a superfície plana $e_{n}=$ 0.831. Utilizando esses valores são calculados: massa efetiva $m_{e f f}=4.209 \times 10^{-5} \mathrm{~kg}$, raio efetivo $r_{\text {eff }}=1.590 \times 10^{-3} \mathrm{~m}$, módulo de Young efetivo $E_{\text {eff }}=37.998 \mathrm{GPa}$, e coeficiente de rigidez não-linear $k_{n, H z}=2.0202 \times 10^{9} \mathrm{~N} / \mathrm{m}^{1.5}$. Os valores para o coeficiente de rigidez linear para a colisão com a parede são apresentados na Tabela 15.

Observa-se na Tabela 14 que os valores dos coeficientes de amortecimentos adimensionais $\left(\nu_{A}^{*}, \nu_{B}^{*}\right)$ são pequenos porque o coeficiente de restituição normal está perto de uma unidade. Para esse caso, ambos os modelos (A e B) resultam em valores similares. 
Tabela 15 - Coeficiente de rigidez normal para o modelo linear: colisão com a parede.

\begin{tabular}{lllll}
\hline Modelo & $\nu_{A, B}^{*}$ & $\delta_{\max L-A, B}^{*}$ & $t_{c, n N L-A, B}^{*}$ & $\bar{\delta}_{n N L-A, B}^{*}$ \\
& & $k_{n}[\mathrm{MN} / \mathrm{m}](\mathrm{Eq} .4 .96)$ & $k_{n}[\mathrm{MN} / \mathrm{m}](\mathrm{Eq} .4 .101)$ & $k_{n}[\mathrm{MN} / \mathrm{m}]$ (Eq.4.104) \\
\hline $\mathrm{A}$ & 0.0658 (Eq.4.58) & 1.0181 & 3.2830 & 0.6356 \\
& & 3.3751 & 3.8421 & 3.5279 \\
$\mathrm{~B}$ & 0.1682 (Eq.4.85) & 1.0101 & 3.2965 & 0.6305 \\
& & 3.4288 & 3.8106 & 3.5856 \\
$\mathrm{~B}$ & 0.1545 (Eq.4.86) & 1.0160 & 3.2897 & 0.6342 \\
& & 3.3895 & 3.8263 & 3.5431 \\
\hline
\end{tabular}

Quando se compara os três métodos para o cálculo de $k_{n}$, Eqs. (4.96), (4.101) e (4.104), recomenda-se como uma estimativa inicial para $k_{n}$ (para o caso examinado aqui, isto é, colisão simples normal entre duas partículas esféricas) a abordagem da sobreposição média (Eq.4.104), embora quaisquer das duas outras abordagens apresentadas (sobreposição máxima ou duração do contato) ou outras disponíveis na literatura são possíveis de serem utilizadas. Dessa forma, para a colisão binária entre duas esferas de vidro, o valor obtido para o coeficiente de rigidez na direção normal para o modelo linear é aproximadamente $k_{n}=2.08 \mathrm{MN} / \mathrm{m}$.

Os valores de $\nu_{B}^{*}$ nas Tabelas 14 e 15 não são exatos pois foram determinados por equações ajustadas (Eqs.4.85 e 4.86). Na Tabela 15, ambos modelos não-lineares (A e B) também resultam em valores similares para o caso analisado, isto é, $e_{n}=0.831 \mathrm{e}$ $v_{0}=1.35 \mathrm{~m} / \mathrm{s}$. Nota-se que o coeficiente de restituição na direção normal para o modelo não-linear B é dependente da velocidade de impacto. Existem diversos modelos não-lineares para estimar os parâmetros de colisão durante o impacto normal (por exemplo, (TSUJI; TANAKA; ISHIDA, 1992), (HUNT; GROSSLEY, 1975), (KUWABARA; KONO, 1987)). Dependendo do problema de contato de partículas, como colisão entre esferas viscoelásticas, um modelo contínuo (como o modelo não-linear B) poderia ser utilizado para evitar atrações não físicas durante o final do impacto. No caso de colisão de esferas viscoelástica, outro modelo com o amortecimento proporcional a raiz quadrada da sobreposição $(p=1 / 2)$ foi proposto por Kuwabara e Kono (1987). Stevens e Hrenya (2005) compararam diversos modelos de esfera suava com experimentos usando partículas de aço em impacto normal. Os dados experimentais obtidos por Stevens e Hrenya (2005) mostram que o tempo de colisão medido no trabalho dos autores está na faixa de $10 \%$ do tempo predito pela teoria de Hertz. Dessa forma, no problema estudado por Foerster et al. (1994) (colisão com a parede, tabela 15), uma estimativa para o coeficiente de rigidez normal pode ser obtida pelo modelo não-linear "A" (Eq. 4.53) ou "B" (Eq. 4.71) em conjunto com a abordagem da sobreposição média (Eq. 4.104), resultando um valor para o coeficiente de rigidez na direção normal de aproximadamente $k_{n}=3.53 \mathrm{MN} / \mathrm{m}$ (modelo "A"), $k_{n}=3.59 \mathrm{MN} / \mathrm{m}$ (modelo "B", Eq. 4.85) ou $k_{n}=3.54 \mathrm{MN} / \mathrm{m}$ (modelo "B", Eq. 4.86) . O algoritmo proposto pela Eq. (4.57) para o cálculo da sobreposição média pode ser utilizado em conjunto com outros modelos não-lineares de contato pelo método da esfera-suave. 


\subsection{Partícula em queda livre}

Nesta seção é analisada a queda livre de uma partícula e seus saltos com a colisão com uma superfície. A Figura 27 mostra o modelo de uma bola caindo sobre uma superfície estacionária. Este problema clássico tem sido aplicado por diversos autores (p.ex. (CHEN; DRUMM; GUIOCHON, 2007), (FLORES et al., 2011), (GARG et al., 2012), (JANKOWSKI, 2005), (YE; LI; ZHU, 2009)). Usando o trabalho de Garg et al. (2012) e Chen, Drumm e Guiochon (2007), analisa-se um partícula de diâmetro de $0.2 \mathrm{~m}$ caindo e batendo sobre uma parede. Os parâmetros da partícula são (CHEN; DRUMM; GUIOCHON, 2007): massa específica $\rho=2600 \mathrm{~kg} / \mathrm{m}^{3}$, módulo de Young da partícula $E=1.6916 \mathrm{MPa}$, módulo de Young da superfície $E_{w}=5.0748 \mathrm{MPa}$, coeficiente de Poisson da partícula e da parede $\sigma=\sigma_{w}=0$, e dois valores para o coeficiente de restituição normal $\left(e_{n}=0.9\right.$ e $\left.e_{n}=0.7\right)$ para a colisão com a superfície. Além dessas propriedades são também utilizados os seguintes valores: altura inicial da queda $h_{0}=0.5 \mathrm{~m}$ e aceleração da gravidade $g=9.81 \mathrm{~m} / \mathrm{s}^{2}$ (ver Tabela 16). A velocidade de impacto da partícula com a superfície é $v_{0}=-\sqrt{2 g\left(h_{0}-r_{p}\right)}=-2.801 \mathrm{~m} / \mathrm{s}$, onde $r_{p}$ é o raio da partícula.

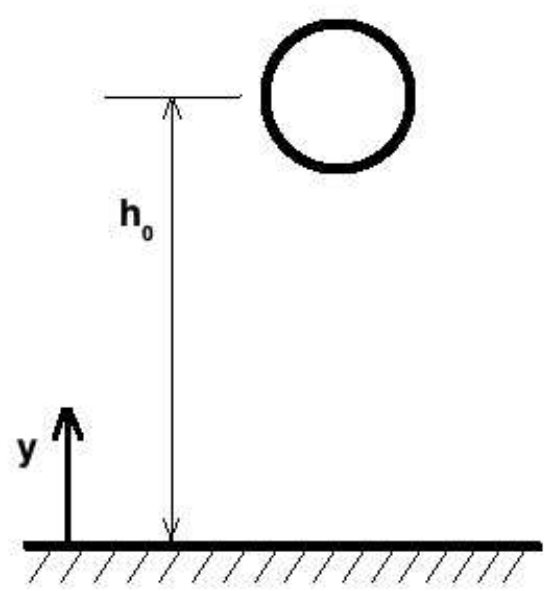

Figura 27 - Esquema da partícula em queda livre.

Tabela 16 - Parâmetros de simulação para a partícula em queda livre.

\begin{tabular}{ll}
\hline Altura da queda & $h=0.5 \mathrm{~m}$ \\
Diâmetro da partícula & $d=0.2 \mathrm{~m}$ \\
Massa específica da partícula & $\rho=2600 \mathrm{~kg} / \mathrm{m}^{3}$ \\
Módulo de Young da partícula & $E=1.6916 \mathrm{MPa}$ \\
Módulo de Young da parede & $E_{w}=5.0748 \mathrm{MPa}$ \\
Coeficiente de restituição partícula-parede & $e_{w}=0.9 \mathrm{e} e_{w}=0.7$ \\
Coeficiente de Poisson partícula e parede & $\sigma_{w}=0$ \\
Aceleração da gravidade & $g=9.81 \mathrm{~m} / \mathrm{s}^{2}$ \\
\hline
\end{tabular}




\subsubsection{Modelo linear}

Nesta seção determina-se a cinemática da partícula durante a colisão usando o modelo linear. Esse modelo requer como dado de entrada o coeficiente de rigidez da mola. O cálculo de $k_{n}$ é feito pelo procedimento descrito na seção 4.3. Utilizando os dados da Tabela 16 são calculados: massa efetiva $m_{\text {eff }}=10.891 \mathrm{~kg}$, raio efetivo $r_{\text {eff }}=0.1 \mathrm{~m}$, módulo de Young efetivo $E_{\text {eff }}=1268700 \mathrm{~Pa}$, e coeficiente de rigidez não-linear $k_{n, H z}=5.3493 \times 10^{5} \mathrm{~N} / \mathrm{m}^{1.5}$. Os valores do coeficiente de rigidez linear são mostrados na Tabela 17.

Tabela 17 - Coeficiente de rigidez normal para o modelo linear: partícula em queda livre.

\begin{tabular}{lllll}
\hline Modelo & $e_{n}$ & $\delta_{\max N L-A, B}^{*}$ & $t_{c, n N L-A, B}^{*}$ & $\bar{\delta}_{N L-A, B}^{*}$ \\
& $\nu_{A, B}^{*}[\mathrm{~N} / \mathrm{m}]($ Eq.4.96) & $k_{n}[\mathrm{~N} / \mathrm{m}]($ Eq.4.101) & $k_{n}[\mathrm{~N} / \mathrm{m}](\mathrm{Eq} .4 .104)$ \\
\hline $\mathrm{A}$ & 0.9 & 1.0492 & 3.2537 & 0.6555 \\
& $0.0375($ Eq.4.58) & $7.6294 \times 10^{4}$ & $8.6899 \times 10^{4}$ & $7.9355 \times 10^{4}$ \\
$\mathrm{~B}$ & 0.9 & 1.0437 & 3.2604 & 0.6520 \\
& $0.0937($ Eq.4.85) & $7.7092 \times 10^{4}$ & $8.6543 \times 10^{4}$ & $8.0199 \times 10^{4}$ \\
$\mathrm{~B}$ & 0.9 & 1.0478 & 3.2564 & 0.6546 \\
& $0.0854($ Eq.4.86) & $7.6498 \times 10^{4}$ & $8.6752 \times 10^{4}$ & $7.9569 \times 10^{4}$ \\
$\mathrm{~A}$ & 0.7 & 0.9578 & 3.3529 & 0.5961 \\
& $0.1261($ Eq.4.58) & $7.2895 \times 10^{4}$ & $8.2795 \times 10^{4}$ & $7.7705 \times 10^{4}$ \\
$\mathrm{~B}$ & 0.7 & 0.9465 & 3.3861 & 0.5889 \\
& $0.3399($ Eq.4.85) & $7.4636 \times 10^{4}$ & $8.1177 \times 10^{4}$ & $7.9637 \times 10^{4}$ \\
$\mathrm{~B}$ & 0.7 & 0.9537 & 3.3744 & 0.5936 \\
& $0.3183($ Eq.4.86) & $7.3520 \times 10^{4}$ & $8.1740 \times 10^{4}$ & $7.8370 \times 10^{4}$ \\
\hline
\end{tabular}

Observa-se na Tabela 17 que, para altos valores do coeficiente de restituição $\left(e_{n}=0.9\right)$, ambos modelos não-lineares (A e B) fornecem pequenos valores dos coeficientes de amortecimento $\left(\nu_{A}^{*}, \nu_{B}^{*}\right)$. Comparando os três métodos para a determinação de $k_{n}$ (Eqs. 4.96, 4.101 e 4.104), o procedimento da sobreposição média (Eq. 4.104) em conjunto com os modelos não-lineares, "A" (Eq.4.53) ou "B" (Eq. 4.71), fornece uma estimativa para o cálculo do coeficiente de rigidez linear, isto é, $k_{n}=7.94 \times 10^{4} \mathrm{~N} / \mathrm{m}$ (modelo "A"), $k_{n}=8.02 \times 10^{4} \mathrm{~N} / \mathrm{m}$ (modelo "B", Eq. 4.85) ou $k_{n}=7.96 \times 10^{4} \mathrm{~N} / \mathrm{m}$ (modelo "B", Eq. 4.86) . Para o coeficiente de restituição de $e_{n}=0.7$, o coeficiente de rigidez também pode ser estimado pelo método da sobreposição média e os modelos não-lineares $k_{n}=7.77 \times 10^{4} \mathrm{~N} / \mathrm{m}$ ("A"), $k_{n}=7.96 \times 10^{4} \mathrm{~N} / \mathrm{m}$ ("B", Eq. 4.85$)$ ou $k_{n}=7.84 \times 10^{4} \mathrm{~N} / \mathrm{m}$ ("B", Eq. 4.86). O método da sobreposição média é vantajoso em relação aos métodos da duração de contato e da sobreposição máxima por ser um método que considera todo o domínio tempo durante a colisão ao invés de usar propriedades instantâneas.

A seguir calcula-se o histórico temporal da posição e da velocidade durante a queda e colisão da partícula. Nas simulações é utilizado o código aberto MFIX ("Multiphase Flow with Interphase eXchanges”) ((SYAMLAL; ROGERS; O’BRIEN, 1993), (SYAMLAL, 1998)) desenvolvido no NETL ("National Energy Technology Laboratory, USA") que tem 
sido amplamente usado para simulações hidrodinâmicas, transferência de calor e massa, e reações químicas em leitos fluidizados borbulhantes e circulantes. A versão do código que calcula o método dos elementos discretos é chamada de MFIX-DEM (ver (GARG et al., 2010)) e possui métodos programados para a determinação das forças de interação entre o fluido e a partícula. O teste para determinar a cinemática da partícula em queda livre, também, é descrito no trabalho desenvolvido por Garg et al. (2012). Os valores utilizados nas simulação são os mostrados na Tabela 16. No cálculo das forças de contato é utilizado o modelo linear. Primeiramente é simulado a partícula em queda livre com o coeficiente de restituição igual a 0.9 . Para esse valor de $e_{n}$ é utilizado o seguinte coeficiente de rigidez linear $k_{n}=7.94 \times 10^{4} \mathrm{~N} / \mathrm{m}$. A Figura 28 ilustra a posição do centro da partícula $y$ e a velocidade da partícula $v$ em função do tempo durante o período de $t=0 \mathrm{~s}$ até $t=1.6 \mathrm{~s}$ ). Com o contato modelado linearmente a partícula salta a cada impacto com a superfície perdendo energia devido às deformações. Observa-se na figura que a duração do impacto ocorre quando os valores da posição do centro da partículas são $y<0.1 \mathrm{~m}$, onde as forças repulsivas atuam. Durante o intervalo total de simulação, $t=1.6 \mathrm{~s}$, a partícula colide com a superfície três vezes.

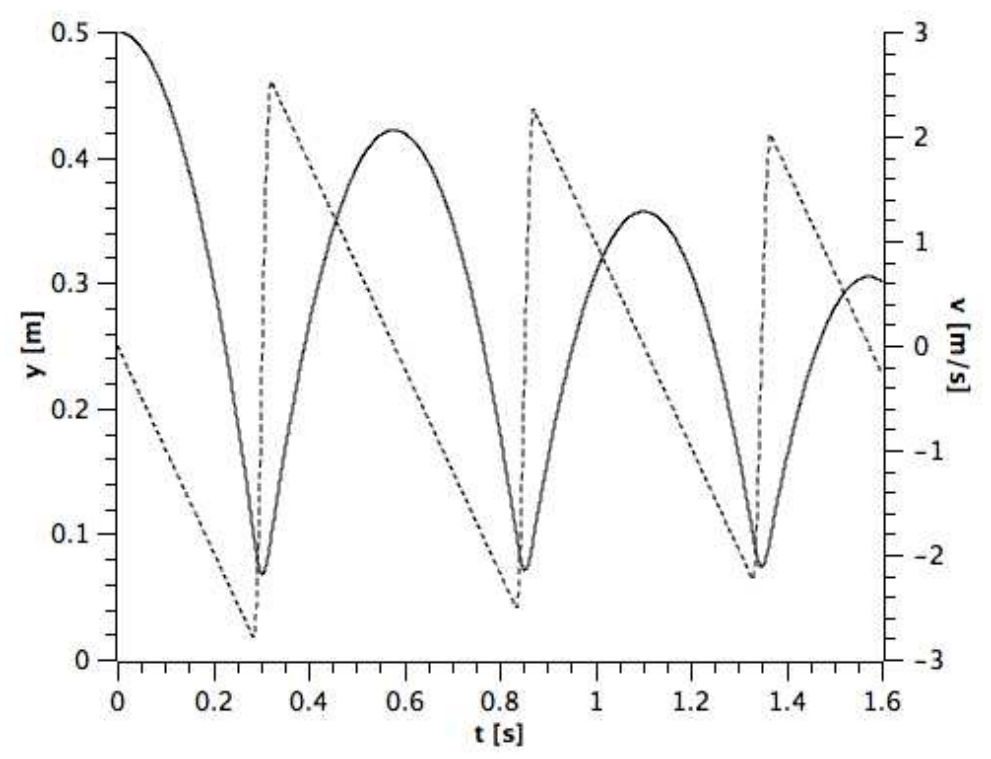

Figura 28 - Modelo linear $\left(k_{n}=7.94 \times 10^{4} \mathrm{~N} / \mathrm{m}\right.$ e $e_{n}=0.9$ : posição do centro da partícula $y$ (linha sólida); velocidade da partícula $v$ (linha tracejada).

A Figura 29 mostra os resultados das simulações para o coeficiente de restituição igual a 0.7 . Nesse caso o coeficiente de rigidez utilizado é $k_{n}=7.77 \times 10^{4} \mathrm{~N} / \mathrm{m}$ conforme determinado anteriormente. Na Figura 29 são plotados os parâmetros cinemáticos do movimento da partícula (posição do centro da partícula y e velocidade da partícula $v$ versus o tempo). De modo similar ao caso analizado anteriormente a partícula bate com a superfície perdendo energia. Como o coeficiente de restituição é muito menor que uma unidade, a partícula salta menos diminuindo a amplitude até permanecer parada no solo. Na Figura 29, observa-se sete colisões da partícula com a superfície. Falcon et al. (1998) 
apresentaram um estudo experimental do comportamento inelástico de uma bola saltando repetidamente em uma superfície horizontal estática.

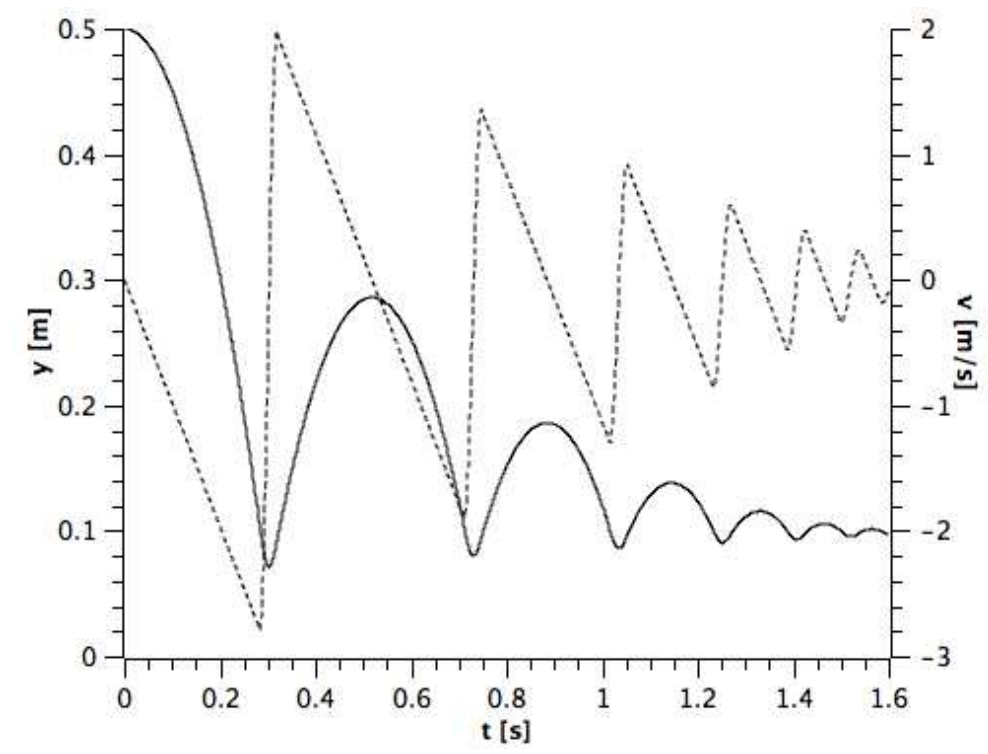

Figura 29 - Modelo linear $\left(k_{n}=7.77 \times 10^{4} \mathrm{~N} / \mathrm{m}\right.$ e $e_{n}=0.7$ : posição do centro da partícula $y$ (linha sólida); velocidade da partícula $v$ (linha tracejada).

\subsubsection{Modelo não-linear}

Como no modelo linear da Seção 4.4.1, os dados das simulações foram extraídos do trabalho de Chen, Drumm e Guiochon (2007). A velocidade da primeira colisão da partícula com a superfície é $v_{0}=-2.801 \mathrm{~m} / \mathrm{s}$. No cálculo das forças de contato dos modelos não-lineares foram utilizadas as Eqs. (4.36), (4.37), (4.45) e (4.46). O parâmetro $p$ das Eqs. (4.45) e (4.46) tem o valor $p=1 / 4$ para o modelo "A" (Eqs. 4.53 e 4.58 ) e $p=3 / 2$ para o modelo "B" (Eqs. 4.71 e 4.86). As Figuras 30 e 31 mostram a posição do centro da partícula $y$ e a velocidade da partículas $v$ versus o tempo (de $t=0 \mathrm{~s}$ até $t=1.6 \mathrm{~s}$ ) para os modelos "A" e "B", respectivamente e $e_{n}=0.9$. Como o coeficiente de restituição tem o valor perto da unidade, os efeitos de amortecimento são pequenos e, também, a elasticidade não-linear não altera as curvas da posição e velocidade da partícula em comparação com o modelo linear (ver Figura 28).

As mesmas propriedades cinemáticas (posição e velocidade) são calculadas para um coeficiente de restituição de 0.7. As Figuras 32 e 33 ilustram essas propriedades para os modelos não-lineares, "A" e "B", respectivamente. Para ambos modelos, a esfera bate na superfície e perde energia mecânica durante o contato. No modelo "A" a partícula colide por seis vezes parando na superfície após essa última colisão. No caso do modelo "B" (Fig. 33) a esfera perde menos energia em cada contato, resultando em um menor número de colisões (cinco) durante o mesmo intervalo de $1.6 \mathrm{~s}$. 


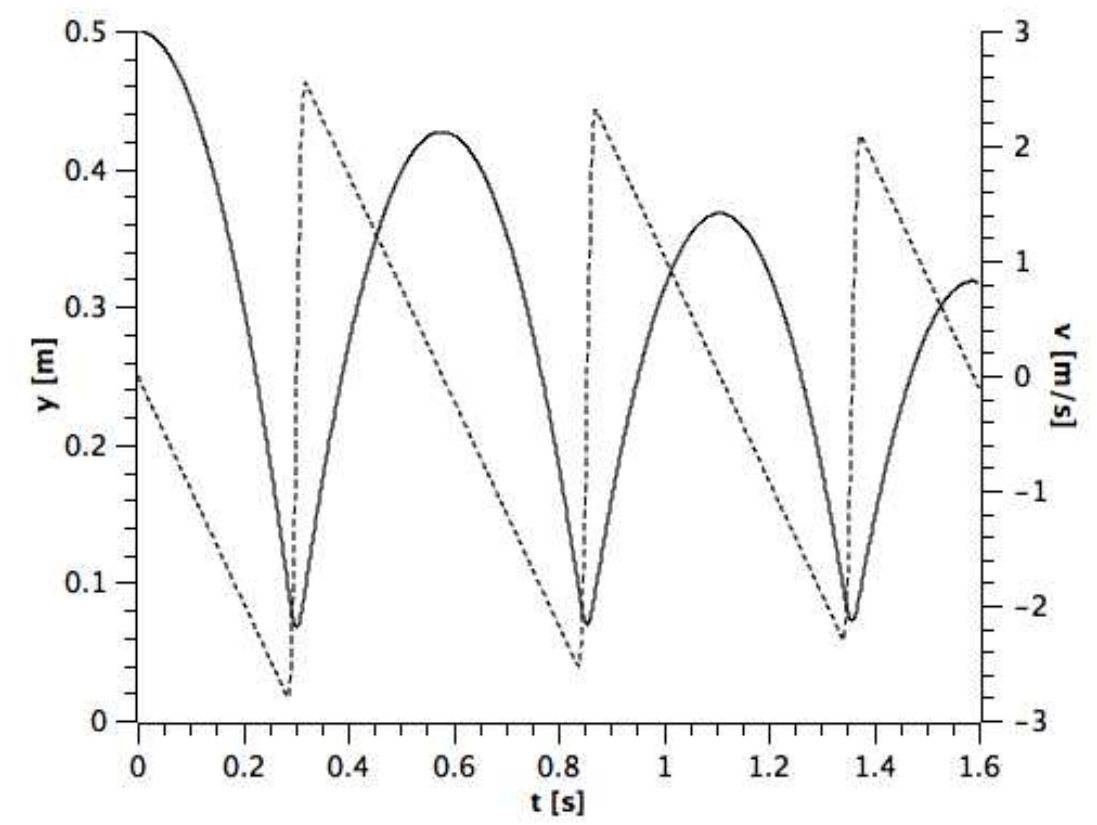

Figura 30 - Modelo não-linear "A" $\left(e_{n}=0.9\right)$ : posição do centro da partícula y (linha sólida); velocidade da partícula $v$ (linha tracejada).

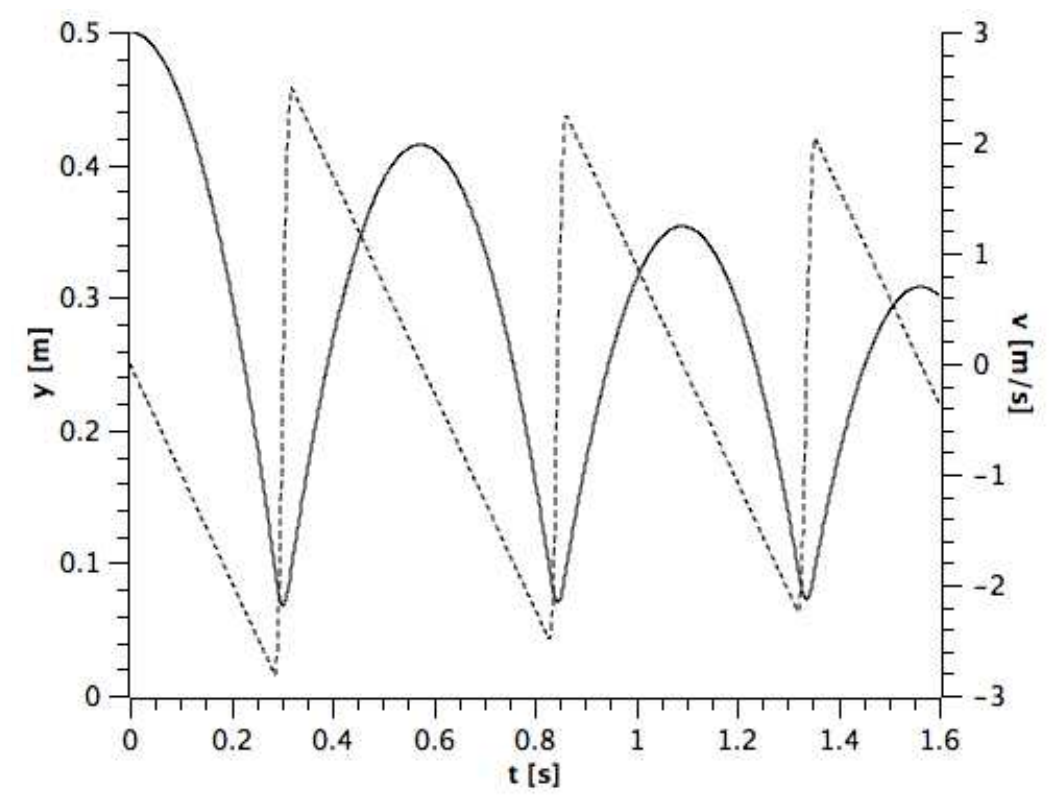

Figura 31 - Modelo não-linear "B" $\left(e_{n}=0.9\right)$ : posição do centro da partícula y (linha sólida); velocidade da partícula $v$ (linha tracejada).

Comparando as Figuras 30,31 e 32, 33 observa-se que as maiores diferenças entre os modelos não-lineares "A" e "B" ocorrem para valores pequenos do coeficiente de restituição (no presente caso para $e_{n}=0.7$ ). Para altos valores as diferenças são muito pequenas.

Observa-se que a perda de energia é menor no modelo "B" produzindo menos impactos e que a duração de cada impacto também é menor nesse modelo. Essa característica é relevante quando se considera sistemas com um número grande de partículas economizando tempo computacional. 


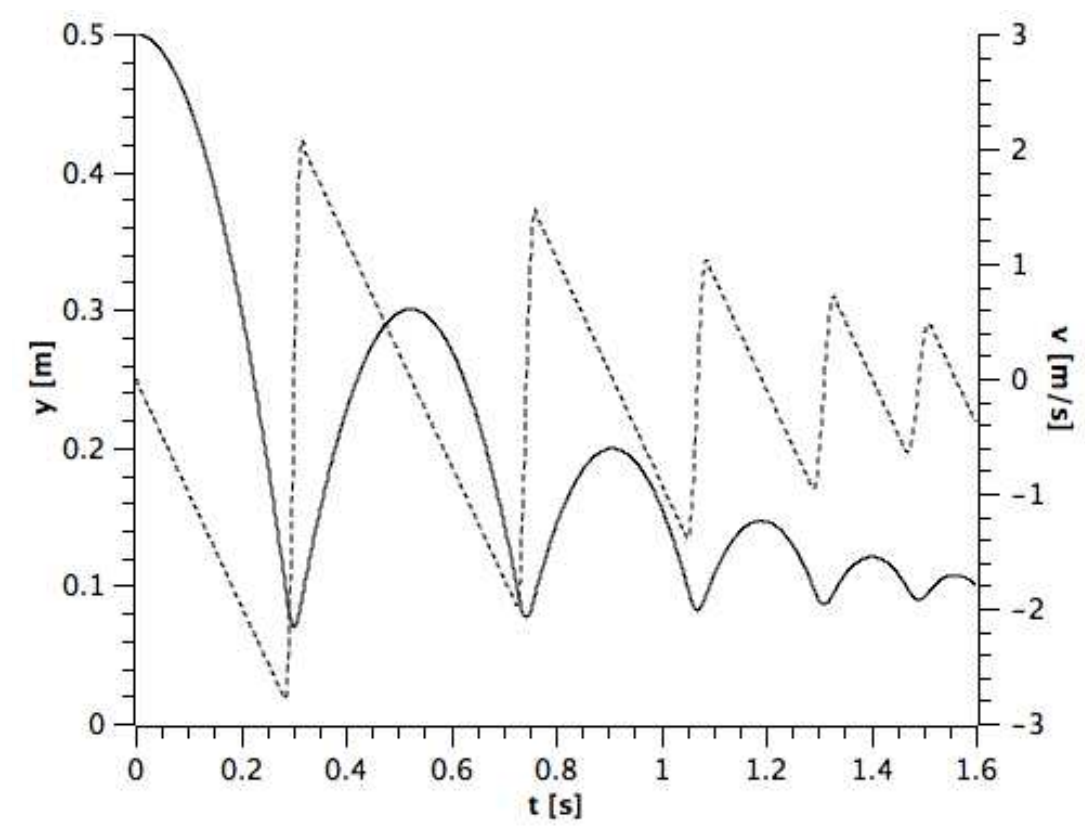

Figura 32 - Modelo não-linear "A" $\left(e_{n}=0.7\right)$ : posição do centro da partícula y (linha sólida); velocidade da partícula $v$ (linha tracejada).

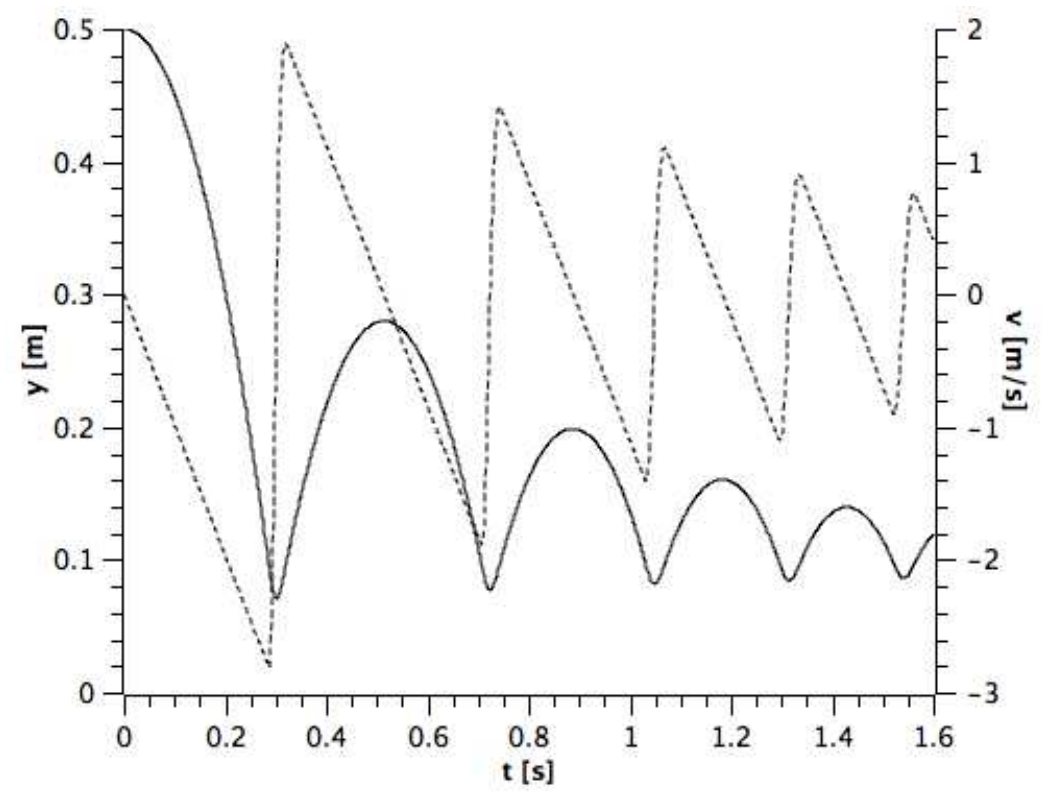

Figura 33 - Modelo não-linear "B" $\left(e_{n}=0.7\right)$ : posição do centro da partícula y (linha sólida); velocidade da partícula $v$ (linha tracejada). 


\section{Sistemas com Fonte de Potência Limitada}

\subsection{Introdução}

No presente capítulo analisa-se um sistema não-ideal do tipo motor elétrico com oscilador mecânico em que não somente o motor afeta o movimento oscilatório mas também o oscilador tem influência no movimento do motor. Esse fenômeno foi descoberto por Sommerfeld (1902) e é baseado no Princípio da Conservação da Energia (ver (SOMMERFELD, 1902), (TIMOSHENKO, 1937)). Conhecido como "Efeito Sommerfeld" o fenômeno foi estudado por Kononenko (1969) em seu livro sobre vibração de sistemas mecânicos com fonte de potência limitada. Arnold Sommerfeld descobriu um "sumidoro de energia" na região de ressonância de uma estrutura excitada por um motor desbalanceado, ou seja, uma grande transferência da energia da fonte para a vibração da estrutura na ressonância ao invés de aumentar a rotação do motor. Quando a potência da fonte aumenta, a estrutura vibra mais e a velocidade de rotação do motor aumenta e quanto mais próxima a rotação do motor estiver da região de ressonância, a fonte deverá fornecer mais potência para aumentar a rotação do motor uma vez que essa energia é transferida para aumentar a amplitude de oscilação da estrutura, ou seja, uma grande aumento da potência da fonte resulta em um pequeno acréscimo na rotação do motor e um grande aumento na amplitude de oscilação do sistema mecânico até que aumentando a potência da fonte resulta no fenômeno de salto com uma diminuição brusca na amplitude de vibração da estrutura e um grande aumento na rotação do motor, sendo esse o fenômeno conhecido como efeito Sommerfeld. O salto também pode ocorrer no sentido inverso, isto é, diminuindo a rotação do motor.

Segundo Kononenko (1969, página 9) uma fonte de energia ideal é aquela que atua em um sistema oscilatório mas não experimenta nenhuma influência recíproca do sistema. Em tais fontes existe uma lei específica de ação no sistema oscilatório independentemente das condições de movimento do sistema, como uma força externa com uma dada frequência e amplitude, uma velocidade constante imposta, ou outra lei de ação sobre o sistema. O efeito de uma fonte de energia ideal sobre um sistema oscilatório pode ser representado por funções explícitas do tempo. Ainda, de acordo com Kononenko (1969), uma fonte de energia não-ideal atua sobre o sistema oscilatório e ao mesmo tempo sofre uma ação recíproca do sistema. Alterações nos parâmetros do sistema podem ser acompanhadas por alterações nas condições de trabalho da fonte de energia. Essa interação torna-se mais ativa quando a fonte de energia tem uma potência muito limitada. Como a influência da fonte de energia não-ideal sobre o sistema oscilatório depende do seu estado de movimento, é impossível expressar essa ação como uma função explícita do tempo. Assim, um sistema 
oscilatório com uma fonte não-ideal deve ser considerado como um sistema autônomo (KONONENKO, 1969).

Diversos autores examinaram o efeito Sommerfeld, tais como Balthazar et al. (2004), Balthazar e Pontes (2005), Cveticanin (2010), Kononenko (1969), Krasnopolskaya e Shvets (1993), Nayfeh e Mook (1976), Sommerfeld (1902), entre outros. Krasnopolskaya e Shvets estudaram e encontraram pela primeira vez caos em sistemas oscilantes com fonte de potência limitada (ver (KRASNOPOLSKAYA; SHVETS, 1990b; KRASNOPOLSKAYA; SHVETS, 1990a; KRASNOPOLSKAYA; SHVETS, 1991; KRASNOPOLSKAYA; SHVETS, 1993)).

Existem várias aplicações que utilizam o impacto para produzir vibrações em sistemas mecânicos, como transportadores vibratórios, máquinas de perfuração, misturadores de pó, entre outros. Diversos autores estudam a influência do impacto na vibração de sistemas, tais como, Liu et al. (2013) que estudou vibro-impacto em um sistema confinado em uma cápsula, Ing et al. (2010) conduziu investigações experimentais e semi-analíticas de um oscilador com impacto, Zukovic e Cveticanin (2009) analisaram o caos em mecanismos mecânicos não-ideais de um sistema com folga, Souza et al. (2005) estudaram uma partícula colidindo as paredes de uma sistema não-ideal e também o controle de sistema em situações caóticas, Moraes et al. (2013) investigaram a influência de fontes ideais e não-ideais sobre a dinâmica de um sistema não-linear com vibro-impacto, Blazejczyk-Okolewska et al. (1999) analisaram a mecânica caótica em sistemas com impacto e atrito, Chatterjee, Mallik e Ghosh (1995) estudaram amortecimento com impacto em sistemas não-lineares, Nordmark (1991) analisou o movimento não-periódico causado pelo limite entre a condição de impacto em um oscilador de impacto, entre outros trabalhos.

No presente capítulo é estudado a vibração devido o impacto de um sistema mecânico não-ideal em uma parede rígida. A modelagem do impacto entre o sistema mecânico e a parede rígida é baseada na hipótese que o impacto entre os corpos ocorre sobre deformações locais. Desta forma, durante o contato o sistema mecânico é modificado sendo composto por outro oscilador não-linear acoplado contendo elementos de mola e de amortecedor. Essa abordagem é similar ao Método dos Elementos Discretos para sistemas particulados modelados com o mecanismo "esfera-suave" (ver capítulo 4). O efeito Sommerfeld é obtido analiticamente e numericamente para o sistema mecânico proposto.

\subsection{Modelo do sistema mecânico não-ideal}

O sistema mecânico não-ideal é modelado como um sistema oscilatório com um motor elétrico desbalanceado localizado adjacente a uma parede rígida (ver Figura 34). O sistema oscilatório tem um massa, $M$, e o motor tem uma massa desbalanceada, $m$, e uma excentricidade, $d$. O momento de inércia de massa das partes rotativas é $J$. O oscilador está 
conectado com uma parede à esquerda através de uma mola linear $(\alpha=1)$ ou não-linear $(\alpha \neq 1)$ com rigidez, $k$, e um amortecedor linear com constante de amortecimento, $c$. $\mathrm{O}$ torque do motor elétrico é função da velocidade angular, $\dot{\phi}$. A distância entre o oscilador e parede de colisão é expressa por $G$. A origem do sistema Cartesiano de coordenadas é localizado na posição em que o sistema mecânico está em repouso. O efeito da força gravitacional atuando na massa, $m$, é desprezado. A energia cinética, $T$, energia potencial, $V$, função de dissipação, $\Phi$, e forças generalizadas, $Q_{x}$ e $Q_{\phi}$ são expressas como

$$
\begin{gathered}
T=\frac{1}{2} M \dot{x}^{2}+\frac{1}{2} m(\dot{x}-d \dot{\phi} \sin (\phi))^{2}+\frac{1}{2} m(d \dot{\phi} \cos (\phi))^{2}+\frac{1}{2} J \dot{\phi}^{2} \\
V=\frac{1}{\alpha+1} k x^{\alpha+1} \\
\Phi=\frac{1}{2} c \dot{x}^{2} \\
Q_{x}=0 ; \quad Q_{\phi}=M_{m}(\dot{\phi})
\end{gathered}
$$

onde $d \ll 1$, os pontos representam as derivadas temporais $\dot{()}=d() / d t$ e $M_{m}$ é o torque do motor elétrico.

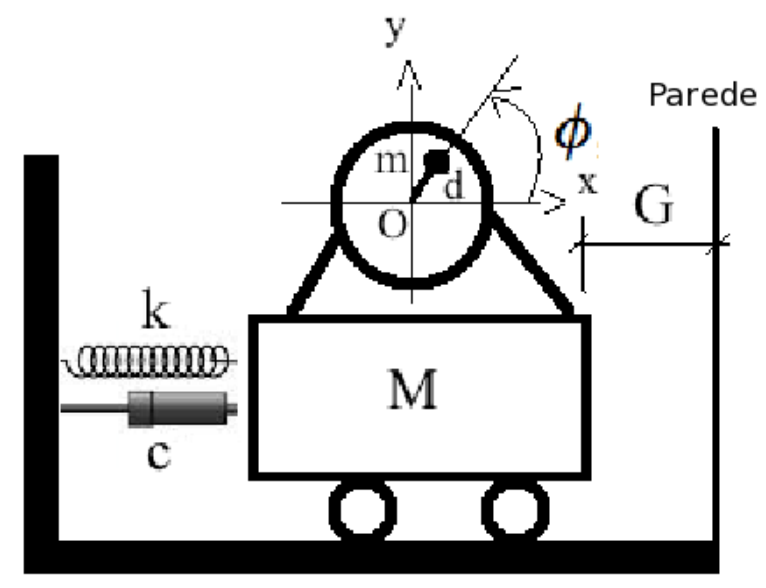

Figura 34 - Modelo do sistema mecânico não-ideal.

As equações de movimento do sistema mecânico são obtidas através das equações de Lagrange:

$$
\begin{aligned}
& \frac{\mathrm{d}}{\mathrm{d} t}\left(\frac{\partial T}{\partial \dot{x}}\right)-\frac{\partial T}{\partial x}+\frac{\partial V}{\partial x}+\frac{\partial \Phi}{\partial \dot{x}}=Q_{x} \\
& \frac{\mathrm{d}}{\mathrm{d} t}\left(\frac{\partial T}{\partial \dot{\phi}}\right)-\frac{\partial T}{\partial \phi}+\frac{\partial V}{\partial \phi}+\frac{\partial \Phi}{\partial \dot{\phi}}=Q_{\phi}
\end{aligned}
$$


resultando nas seguintes equações diferenciais:

$$
\begin{array}{r}
(M+m) \ddot{x}+c \dot{x}+k x|x|^{\alpha-1}+F_{I}=m d\left(\ddot{\phi} \sin (\phi)+\dot{\phi}^{2} \cos (\phi)\right) \\
\left(J+m d^{2}\right) \ddot{\phi}=m d \ddot{x} \sin (\phi)+M_{m}(\dot{\phi})
\end{array}
$$

onde $F_{I}$ é a força de impacto. No presente trabalho durante o impacto o sistema mecânico será acoplado a outro oscilador para o cálculo da força de impacto. A colisão ocorre na direção normal e considerando que o impacto entre os corpos ocorre em um breve intervalo de tempo, desprezando as forças não-conservativas, segundo a Eq. (4.36), tem-se que $(\beta=3 / 2)$ :

$$
\begin{aligned}
& F_{I}=k_{I} \hat{x}|\hat{x}|^{\beta-1}=k_{I} \hat{x}^{3 / 2}, \quad \text { se } \quad x>G>0 \\
& F_{I}=0, \quad \text { se } \quad x \leq G,
\end{aligned}
$$

onde a rigidez $k_{I} \gg k$ e $\hat{x}=x-G$.

O movimento do motor elétrico de corrente contínua é governando por equações elétricas e mecânicas (ver, por exemplo, (BELATO et al., 2001)):

$$
\begin{array}{r}
L \frac{\mathrm{d} i(t)}{\mathrm{d} t}=V(t)-R i(t)-K_{E} \dot{\phi} \\
M_{m}=K_{T} i(t)-J_{m} \ddot{\phi}-c_{m} \dot{\phi}-T_{f}
\end{array}
$$

onde $L$ é a indutância térmica, $i(t)$ é a corrente elétrica, $V(t)$ é a voltagem, $R$ é a resistência elétrica, $K_{E}$ é a constante da voltagem, $K_{T}$ é a constante do torque, $J_{m}$ é o momento de inércia de massa do rotor, $c_{m}$ é o coeficiente das perdas internas e $T_{f}$ é a constante de atrito do motor. Seguindo o trabalho de Belato et al. (2001), foi utilizado um modelo simplificado do motor elétrico considerando $c_{m}=0, T_{f}=0$, e sendo a constante elétrica de tempo $L / R$ menor do que a constante de tempo mecânica $R J / K_{E} K_{T}$, as equações elétrica e mecânica simplificadas do motor para uma voltagem constante são escritas como:

$$
\begin{array}{r}
V=R i-K_{E} \dot{\phi} \\
M_{m}=K_{T} i-J_{m} \ddot{\phi}
\end{array}
$$

Considerando que o momento de inércia de massa do rotor, $J_{m}$, está incluído no momento de inércia das partes rotativas, $J$, o torque do motor, $M_{m}$, é expresso por:

$$
M_{m}(\dot{\phi})=V_{m}^{*}-C_{m}^{*} \dot{\phi}
$$


onde $V_{m}{ }^{*}=K_{T} V / R$ e $C_{m}{ }^{*}=K_{E} K_{T} / R$ são parâmetros característicos do motor.

Para uma voltagem nominal $V_{0}$ pode-se escrever:

$$
M_{m, 0}(\dot{\phi})=M_{0}\left(1-\frac{\dot{\phi}}{\Omega_{0}}\right)
$$

onde $M_{0}=K_{T} V_{0} / R$ é a constante de torque do motor, $\Omega_{0}=V_{0} / K_{E}$ é a velocidade angular (sem carga) constante do motor (ver (DIMENTBERG et al., 1997)).

A solução das equações de movimento é obtida com as seguintes condições iniciais

$$
x(0)=x_{0}, \quad \dot{x}(0)=0, \quad \phi(0)=0, \quad \dot{\phi}(0)=\omega_{0} .
$$

A coordenada espacial $x$ e o tempo $t$ são normalizados de acordo com:

$$
z=x / l_{0} \quad \tau=\Omega_{0} t,
$$

onde $l_{0}$ é o comprimento inicial não-deformado da mola. Usando as relações (5.13) nas equações diferenciais (5.6), as equações de movimento adimensionalizadas são expressas por (ver, p.ex., (CVETICANIN; ZUKOVIC, 2015a)):

$$
\begin{array}{r}
z^{\prime \prime}+\varepsilon \gamma z^{\prime}+f_{1}(z)=\varepsilon q_{1}\left(\phi^{\prime \prime} \sin \phi+\phi^{\prime 2} \cos \phi\right) \\
\phi^{\prime \prime}=\varepsilon\left(q_{2} z^{\prime \prime} \sin \phi+M_{1}\left(\phi^{\prime}\right)\right)
\end{array}
$$

e a força conservativa adimensional $f_{1}(z)$ é dada por:

$$
\begin{aligned}
& f_{1}(z)=p^{2} z|z|^{\alpha-1}+p_{I}{ }^{2} \hat{z}^{3 / 2}, \quad \text { se } \quad z>z^{*}>0 \\
& f_{1}(z)=p^{2} z|z|^{\alpha-1}, \quad \text { se } \quad z \leq z^{*},
\end{aligned}
$$

onde

$$
\begin{array}{r}
p=\frac{\omega^{*}}{\Omega_{0}}, \quad \omega^{* 2}=\frac{k l_{0}{ }^{\alpha-1}}{M+m}, \quad p_{I}=\frac{\omega_{I}^{*}}{\Omega_{0}}, \quad \omega_{I}^{* 2}=\frac{k_{I} l_{0}{ }^{1 / 2}}{M+m}, \quad z^{*}=\frac{G}{l_{0}}, \\
\hat{z}=\frac{\hat{x}}{l_{0}}=z-z^{*} \quad q_{1}{ }^{*}=\varepsilon q_{1}=\left(\frac{m}{M+m}\right)\left(\frac{d}{l_{0}}\right) \quad q_{2}^{*}=\varepsilon q_{2}=\left(\frac{d}{l_{0}}\right) \frac{m l_{0}{ }^{2}}{\left(J+m d^{2}\right)}, \\
\gamma^{*}=\varepsilon \gamma=\left(\frac{m}{M+m}\right)\left(\frac{c}{\Omega_{0} m}\right) \quad M_{1}\left(\phi^{\prime}\right)^{*}=\varepsilon M_{1}\left(\phi^{\prime}\right)=\frac{V_{m}{ }^{*}-C_{m}{ }^{*} \Omega_{0} \phi^{\prime}}{\left(J+m d^{2}\right) \Omega_{0}{ }^{2}}
\end{array}
$$

com as condições iniciais adimensionalizadas:

$$
z(0)=x_{0} / l_{0}, \quad z^{\prime}(0)=0, \quad \phi(0)=0, \quad \phi^{\prime}(0)=\omega_{0} / \Omega_{0}
$$

Nas Eqs. (5.14) a linha denota a derivada em relação ao tempo adimensional $\tau$ e pelas características dos parâmetros físicos do sistema as variáveis $q_{1}{ }^{*}, q_{2}{ }^{*}, \gamma^{*}, M_{1}\left(\phi^{\prime}\right)^{*}$ têm 
valores pequenos em comparação a unidade. Dessa forma essas variáveis são tratadas como produtos de um pequeno parâmetro positivo $\varepsilon \ll 1$. Usando a expressão (5.14) obtém-se:

$$
\begin{array}{r}
z^{\prime \prime}+f_{1}(z)=-\varepsilon\left(\gamma z^{\prime}-q_{1} \phi^{\prime 2} \cos \phi\right) \\
\phi^{\prime \prime}=\varepsilon\left(M_{1}\left(\phi^{\prime}\right)-q_{2} f_{1}(z) \sin \phi\right)
\end{array}
$$

A solução das equações diferenciais (5.18) é obtida usando o procedimento descrito em Cveticanin e Zukovic (2015a). Se o parâmetro $\varepsilon=0$, as equações (5.18) são transformadas em duas equações diferenciais desacopladas da forma:

$$
\begin{aligned}
z^{\prime \prime}+f_{1}(z) & =0, \\
\phi^{\prime \prime} & =0 .
\end{aligned}
$$

Quando o sistema não está em contato com a parede e a função $f_{1}(z)$ é linear a primeira equação de (5.19) tem solução exata na forma de uma função trigonométrica. Quando o sistema está em contato com a parede tem-se que $p_{I} \gg p$ de forma que se pode aproximar a função $f_{1}$ por:

$$
\begin{aligned}
& f_{1}(z) \approx p_{I}^{2} \hat{z}^{3 / 2}, \quad \text { se } \quad z>z^{*}>0, \\
& f_{1}(z)=p^{2} z|z|^{\alpha-1}, \quad \text { se } \quad z \leq z^{*},
\end{aligned}
$$

Considerando o Capítulo 3 da presente tese e os trabalhos de Zukovic e Cveticanin (2009), Cveticanin e Pogany (2012), Cveticanin e Zukovic (2015a), entre outros, pode-se aproximar a solução da primeira equação de (5.19) por uma solução do tipo trigonométrica que representa satisfatoriamente a solução exata:

$$
\begin{array}{r}
z=a \cos (\omega(a) t+\xi), \\
\phi^{\prime}=\Omega,
\end{array}
$$

onde os parâmetros $a, \xi$, e $\Omega$, são constantes que devem satisfazer as condições iniciais (5.17). A frequência de vibração $\omega$ depende da amplitude de vibração $a$ (ver Capítulo 3). O procedimento utilizado na presente seção, para encontrar a solução da equação diferencial de segunda ordem levemente não-linear, foi proposto por originalmente por Krylov e Bogoliubov (1947). Seja o caso ressonante (ver Kononenko (1969)), quando a diferença entre a frequência da vibração do sistema, $\omega$, e a frequência de excitação, $\Omega$ são pequenas, isto é,

$$
\Omega-\omega(a)=\varepsilon \sigma
$$

onde $\varepsilon \sigma \ll 1$. Utilizando a Eq. (5.22) e o procedimento de Krylov e Bogoliubov (1947), a solução (5.21) e a sua primeira derivada são

$$
\begin{array}{r}
z=a \cos (\phi+\xi), \\
z^{\prime}=-a \Omega \sin (\phi+\xi),
\end{array}
$$


em que a solução do sistema (5.18) requer uma pertubação das equações (5.23), isto é, ver Cveticanin e Zukovic (2015a):

$$
z=a(\tau) \cos (\phi(\tau)+\xi(\tau))
$$

e

$$
z^{\prime}=-a(\tau) \Omega(\tau) \sin (\phi(\tau)+\xi(\tau))
$$

A primeira derivada da Eq. (5.24) é dada por:

$$
z^{\prime}=a^{\prime} \cos (\phi+\xi)-a\left(\Omega+\xi^{\prime}\right) \sin (\phi+\xi),
$$

e comparando (5.26) com a equação (5.25), conclui-se que:

$$
a^{\prime} \cos (\phi+\xi)-a \xi^{\prime} \sin (\phi+\xi)=0 \text {. }
$$

A segunda derivada temporal da Eq. (5.24) resulta em:

$$
z^{\prime \prime}=-a^{\prime} \Omega \sin (\phi+\xi)-a \Omega\left(\Omega+\xi^{\prime}\right) \cos (\phi+\xi)-a \Omega^{\prime} \sin (\phi+\xi),
$$

e, portanto, substituindo as Eqs. (5.24), (5.25), e (5.28) na primeira equação de (5.18), obtém-se:

$$
-a^{\prime} \Omega \sin (\phi+\xi)-a \Omega\left(\Omega+\xi^{\prime}\right) \cos (\phi+\xi)-a \Omega^{\prime} \sin (\phi+\xi)+f_{1}\left(a(\cos (\phi+\xi))=\varepsilon f_{2},\right.
$$

onde $f_{2}=\gamma a \Omega \sin (\phi+\xi)+q_{1} \phi^{\prime 2} \cos \phi=\gamma a \Omega \sin (\phi+\xi)+q_{1} \Omega^{2} \cos \phi$.

A solução aproximada (5.21) satisfaz aproximadamente a primeira equação de (5.19) obtendo-se a seguinte relação:

$$
-a \omega^{2} \cos (\phi+\xi)+f_{1}(a(\cos (\phi+\xi)) \approx 0 .
$$

e usando a Eq. (5.30) reescreve-se a Eq. (5.29) como:

$$
-a^{\prime} \Omega \sin (\phi+\xi)-a\left(\Omega^{2}-\omega^{2}\right) \cos (\phi+\xi)-a \Omega \xi^{\prime} \cos (\phi+\xi)-a \Omega^{\prime} \sin (\phi+\xi)=\varepsilon f_{2},
$$

Utilizando a Eq. (5.27) na Eq. (5.31) obtém-se:

$$
a^{\prime}=-\frac{\varepsilon}{\Omega} f_{2} \sin (\phi+\xi)-a \frac{\left(\Omega^{2}-\omega^{2}\right)}{\Omega} \sin (\phi+\xi) \cos (\phi+\xi)-\frac{\Omega^{\prime}}{\Omega} a \sin ^{2}(\phi+\xi),
$$

A segunda equação de (5.18) pode ser transformada em duas equações de primeira ordem utilizando a Eq. (5.21), isto é,

$$
\begin{gathered}
\Omega^{\prime}=\varepsilon f_{3}, \\
\phi^{\prime}=\Omega,
\end{gathered}
$$


onde $f_{3}=M_{1}(\Omega)-q_{2} a \omega^{2} \cos (\phi+\xi) \sin \phi$

Além das Eqs. (5.33) as duas equações a seguir formam um sistema de primeira ordem com quatro equações correspondente ao sistema das equações (5.18):

$$
\begin{array}{r}
a^{\prime}=-\frac{\varepsilon}{\Omega}\left[f_{2}+f_{3} a \sin (\phi+\xi)\right] \sin (\phi+\xi)-a \frac{\left(\Omega^{2}-\omega^{2}\right)}{\Omega} \sin (\phi+\xi) \cos (\phi+\xi), \\
a \xi^{\prime}=-\frac{\varepsilon}{\Omega}\left[f_{2}+f_{3} a \sin (\phi+\xi)\right] \cos (\phi+\xi)-a \frac{\left(\Omega^{2}-\omega^{2}\right)}{\Omega} \cos ^{2}(\phi+\xi),
\end{array}
$$

As Eqs. (5.33) e (5.34) são complexas e portanto será utilizado um procedimento para obter soluções aproximadas dessas equações. As ressonâncias serão eliminadas usando $\Omega(\tau)-\omega(a)=\varepsilon \sigma$ e um procedimento de média (ver (KRYLOV; BOGOLIUBOV, 1947), (NAYFEH, 2004), (ZUKOVIC; CVETICANIN, 2009), (CVETICANIN; ZUKOVIC, 2015a), entre outros). As equações médias serão obtidas sobre um período de vibração. Substituindo as expressões de $f_{2}$ e $f_{3}$ nas Eqs. (5.34) e (5.33) resulta:

$$
\begin{array}{r}
a^{\prime}=-\varepsilon \gamma a \sin ^{2}(\phi+\xi)-\varepsilon q_{1} \Omega \cos \phi \sin (\phi+\xi)-\frac{\varepsilon}{\Omega} M_{1}(\Omega) a \sin ^{2}(\phi+\xi) \\
+\frac{\varepsilon}{\Omega} q_{2} a^{2} \omega^{2} \cos (\phi+\xi) \sin ^{2}(\phi+\xi) \sin \phi-a \frac{\left(\Omega^{2}-\omega^{2}\right)}{\Omega} \sin (\phi+\xi) \cos (\phi+\xi), \\
a \xi^{\prime}=-\varepsilon \gamma a \sin (\phi+\xi) \cos (\phi+\xi)-\varepsilon q_{1} \Omega \cos \phi \cos (\phi+\xi)-\frac{\varepsilon}{\Omega} M_{1}(\Omega) a \sin (\phi+\xi) \cos (\phi+\xi) \\
+\frac{\varepsilon}{\Omega} q_{2} a^{2} \omega^{2} \cos ^{2}(\phi+\xi) \sin (\phi+\xi) \sin \phi-a \frac{\left(\Omega^{2}-\omega^{2}\right)}{\Omega} \cos ^{2}(\phi+\xi),
\end{array}
$$

e

$$
\begin{array}{r}
\Omega^{\prime}=\varepsilon M_{1}(\Omega)-\varepsilon q_{2} a \omega^{2} \cos (\phi+\xi) \sin \phi, \\
\phi^{\prime}=\Omega .
\end{array}
$$

As Eqs. (5.35) e (5.36) são resolvidas usando o procedimento das médias de Krylov e Bogoliubov (1947). A média das funções é feita sobre o período de $2 \pi$ da variável $\phi$, resultando em:

$$
\begin{array}{r}
a^{\prime}=-\varepsilon\left(\frac{a M_{1}(\Omega)}{2 \Omega}+\frac{a \gamma}{2}+\frac{q_{1} \Omega}{2} \sin (\xi)+\frac{q_{2} a^{2} \omega^{2}}{8 \Omega} \sin (\xi)\right) \\
\xi^{\prime}=-\frac{\left(\Omega^{2}-\omega^{2}\right)}{2 \Omega}-\frac{\varepsilon q_{1} \Omega}{2 a} \cos (\xi)+\frac{\varepsilon q_{2} a \omega^{2}}{8 \Omega} \cos (\xi) \\
\Omega^{\prime}=\varepsilon\left(M_{1}(\Omega)+\frac{q_{2} a \omega^{2}}{2} \sin \xi\right),
\end{array}
$$

Considerando a resposta em regime permanente as Eqs. (5.37) têm a seguinte forma:

$$
\begin{array}{r}
-\left(\frac{a M_{1}(\Omega)}{\Omega}+a \gamma\right)=\left(q_{1} \Omega+\frac{q_{2} a^{2} \omega^{2}}{4 \Omega}\right) \sin (\xi) \\
\frac{\left(\Omega^{2}-\omega^{2}\right)}{\Omega} a=\varepsilon\left(-q_{1} \Omega+\frac{q_{2} a^{2} \omega^{2}}{4 \Omega}\right) \cos (\xi) \\
M_{1}(\Omega)=-\frac{q_{2} a \omega^{2}}{2} \sin \xi,
\end{array}
$$


Caso o momento de inércia de massa das partes da rotativa (que inclui o momento de inércia do rotor), $J$, tenha um valor grande, os parâmetros adimensionais das Eqs. (5.16) podem ser escritos como $q_{2}{ }^{*}=\varepsilon^{2} q_{2}$ e $M_{1}\left(\phi^{\prime}\right)^{*}=\varepsilon^{2} M_{1}\left(\phi^{\prime}\right)$. Com essa condição a Eq. (5.33) é reescrita como:

$$
\begin{array}{r}
\Omega^{\prime}=\varepsilon^{2} f_{3}, \\
\phi^{\prime}=\Omega,
\end{array}
$$

e a Eq. (5.32) é dada por:

$$
a^{\prime}=-\frac{\varepsilon}{\Omega} f_{2} \sin (\phi+\xi)-a \frac{\left(\Omega^{2}-\omega^{2}\right)}{\Omega} \sin (\phi+\xi) \cos (\phi+\xi)-\frac{\varepsilon^{2} f_{3}}{\Omega} a \sin ^{2}(\phi+\xi),
$$

Desprezando os termos de ordem $\mathcal{O}\left(\varepsilon^{2}\right)$ obtém-se

$$
\begin{array}{r}
a^{\prime}=-\frac{\varepsilon}{\Omega} f_{2} \sin (\phi+\xi)-a \frac{\left(\Omega^{2}-\omega^{2}\right)}{\Omega} \sin (\phi+\xi) \cos (\phi+\xi), \\
a \xi^{\prime}=-\frac{\varepsilon}{\Omega} f_{2} \cos (\phi+\xi)-a \frac{\left(\Omega^{2}-\omega^{2}\right)}{\Omega} \cos ^{2}(\phi+\xi),
\end{array}
$$

e substituindo $f_{2}$ e reescrevendo as equações (5.35) e (5.36), tem-se:

$$
\begin{gathered}
a^{\prime}=-\varepsilon \gamma a \sin ^{2}(\phi+\xi)-\varepsilon q_{1} \Omega \cos \phi \sin (\phi+\xi)-a \frac{\left(\Omega^{2}-\omega^{2}\right)}{\Omega} \sin (\phi+\xi) \cos (\phi+\xi), \\
a \xi^{\prime}=-\varepsilon \gamma a \sin (\phi+\xi) \cos (\phi+\xi)-\varepsilon q_{1} \Omega \cos \phi \cos (\phi+\xi)-a \frac{\left(\Omega^{2}-\omega^{2}\right)}{\Omega} \cos ^{2}(\phi+\xi),
\end{gathered}
$$

e

$$
\begin{array}{r}
\Omega^{\prime}=\varepsilon^{2} M_{1}(\Omega)-\varepsilon^{2} q_{2} a \omega^{2} \cos (\phi+\xi) \sin \phi, \\
\phi^{\prime}=\Omega .
\end{array}
$$

E de forma semelhante que as Eqs. (5.37) a média sobre o período de vibração resulta em:

$$
\begin{array}{r}
a^{\prime}=-\varepsilon\left(\frac{a \gamma}{2}+\frac{q_{1} \Omega}{2} \sin (\xi)\right) \\
\xi^{\prime}=-\frac{\left(\Omega^{2}-\omega^{2}\right)}{2 \Omega}-\frac{\varepsilon q_{1} \Omega}{2 a} \cos (\xi) \\
\Omega^{\prime}=\varepsilon^{2}\left(M_{1}(\Omega)+\frac{q_{2} a \omega^{2}}{2} \sin \xi\right),
\end{array}
$$

e a resposta em regime permanente de (5.44) é:

$$
\begin{array}{r}
-a \gamma=q_{1} \Omega \sin (\xi) \\
\frac{\left(\Omega^{2}-\omega^{2}\right)}{\Omega} a=-\varepsilon q_{1} \Omega \cos (\xi) \\
M_{1}(\Omega)=-\frac{q_{2} a \omega^{2}}{2} \sin \xi
\end{array}
$$


Eliminando $\xi$ das Eqs. (5.45) obtém-se duas relações para o gráfico da amplitude em função da frequência, isto é, $a-\Omega$ :

$$
\left(q_{1} \Omega\right)^{2}=a^{2}\left[\gamma^{2}+\frac{1}{\varepsilon^{2}}\left(\frac{\left(\Omega^{2}-\omega^{2}\right)}{\Omega}\right)^{2}\right]
$$

e

$$
a^{2} \gamma \frac{\omega^{2}}{\Omega}=2 \frac{q_{1}}{q_{2}} M_{1}(\Omega),
$$

em que a solução das equações (5.46) e (5.47) depende da frequência do oscilador (linear ou não-linear durante o impacto) e do torque do motor. A frequência exata de um oscilador conservativo com não-linearidade não-inteira é dada por (ver (CVETICANIN, 2009b) e (CVETICANIN; POGANY, 2012)):

$$
\omega=\omega_{\alpha} \sqrt{p^{* 2}} a^{(\alpha-1) / 2}
$$

onde

$$
\omega_{\alpha}=\sqrt{\frac{\alpha+1}{2}} \frac{\sqrt{\pi} \Gamma\left(\frac{3+\alpha_{1}}{2\left(\alpha_{1}+1\right)}\right)}{\Gamma\left(\frac{1}{\alpha_{1}+1}\right)},
$$

onde $p^{*}=p$ se $z \leq z^{*}$ ou $p^{*}=p_{I}$ se $z>z^{*}$ (a aproximação 5.20 com $\hat{z}=z^{*}$ fornece a posição relativa) e $\Gamma$ é a função gamma. No caso $p^{*}=p_{I}$ e com as Eqs. (5.46), (5.48), a amplitude relativa é aproximadamente nula e a amplitude (total) do movimento à direita é aproximadamente $z^{*}$. O período exato de vibração é dado pela Eq. (3.48). Caso $\alpha=1$ tem-se que $\omega_{1}=1$ e a frequência $\omega^{2}=p^{* 2}$ e para $\alpha=3 / 2$ obtém-se $\omega_{3 / 2}=0.95469$ e $\omega^{2}=0.91144 p^{* 2} a^{1 / 2}$. O torque do motor pode ser reescrito como

$$
M_{1}(\Omega)=M_{2}-C_{m} \Omega
$$

onde $M_{2}=\frac{V_{m}{ }^{*}}{\varepsilon^{2}\left(J+m d^{2}\right) \Omega_{0}{ }^{2}}$ é um parâmetro adimensional de controle que é função da voltagem do motor elétrico e $C_{m}=\frac{C_{m}{ }^{*}}{\varepsilon^{2}\left(J+m d^{2}\right) \Omega_{0}}$.

Substituindo as Eqs. (5.48) e (5.50) nas Eqs. (5.46) e (5.47) resulta em:

$$
\left(q_{1} \Omega\right)^{2}=a^{2}\left[\gamma^{2}+\frac{1}{\varepsilon^{2}}\left(\frac{\left(\Omega^{2}-\omega_{\alpha}^{2} p^{* 2} a^{(\alpha-1)}\right)}{\Omega}\right)^{2}\right]
$$

e

$$
a^{2} \gamma \frac{\omega_{\alpha}^{2} p^{* 2} a^{(\alpha-1)}}{\Omega}=2 \frac{q_{1}}{q_{2}}\left(M_{2}-C_{m} \Omega\right),
$$

Para se determinar os pontos característicos nas curvas $a-\Omega$ onde o efeito do salto ocorre deve-se igualar os gradientes $d a / d \Omega$ para as curvas dadas pelas equações (5.51) e (5.52) (ver (CVETICANIN; ZUKOVIC, 2015b)). Como a solução para se obter o ponto característico $\left(a_{P}, \Omega_{P}\right)$ é não-linear e de alta complexidade, Cveticanin e Zukovic (2015b) sugerem um procedimento aproximado para calcular um ponto $\left(a_{P}^{\prime}, \Omega_{P}^{\prime}\right)$, onde a bifurcação 
da Eq.(5.51) aparece de tal forma que $P^{\prime}$ está próximo ao ponto exato $P$. Dessa forma, usando a Eq. (5.48) a frequência no ponto de pico é dada por :

$$
\Omega_{P^{\prime}}=\omega_{P^{\prime}}=\omega_{\alpha} \sqrt{p^{* 2}} a_{P^{\prime}}(\alpha-1) / 2,
$$

Substituindo a Eq.(5.53) na Eq. (5.51) a amplitude de pico, $a_{P^{\prime}}$, é dada por:

$$
a_{P^{\prime}}=\left(\frac{\gamma}{\omega_{\alpha} \sqrt{p^{* 2}} q_{1}}\right)^{\frac{2}{\alpha-3}}
$$

e usando a Eq. (5.54) na Eq. (5.53) obtém-se a frequência de pico, $\Omega_{P^{\prime}}$ :

$$
\Omega_{P^{\prime}}=\left(\frac{\gamma}{q_{1}}\right)^{\frac{\alpha-1}{\alpha-3}}\left(\omega_{\alpha} \sqrt{p^{* 2}}\right)^{\frac{2}{3-\alpha}}
$$

Usando as Eqs. (5.54) e (5.55) com a Eq. (5.52) obtém-se o valor do parâmetro de controle do torque do motor, $M_{2 P^{\prime}}$ :

$$
M_{2 P^{\prime}}=C_{m}\left(\frac{\gamma}{q_{1}}\right)^{\frac{\alpha-1}{\alpha-3}}\left(\omega_{\alpha} \sqrt{p^{* 2}}\right)^{\frac{2}{3-\alpha}}+\frac{q_{2}}{2}\left(\frac{\gamma}{q_{1}}\right)^{\frac{2 \alpha}{\alpha-3}}\left(\omega_{\alpha} \sqrt{p^{* 2}}\right)^{\frac{6}{3-\alpha}}
$$

e para esse valor de $M_{2 P^{\prime}}$ o efeito Sommerfeld aparece.

A figura 35 (retirada do artigo (ZUKOVIC; CVETICANIN, 2009)) mostra um exemplo de uma curva característica da intersecção entre o motor (A, B, C) e a curva do movimento do sistema mecânico em regime permanente. As curvas $\mathrm{A}$ e $\mathrm{C}$ definem o fenômeno do salto sendo dois o número de soluções em regime para essas curvas. Para as curvas entre A e C (como a curva B) existem 3 soluções em regime. As curvas externas as curvas A e C apresentam somente uma solução em regime, isto é, para valores pequenos ou altos de $M_{2}$. As soluções na linha contínua são estáveis e as na linha tracejada são instáveis.

A figura 36 (retirada do artigo (CVETICANIN; ZUKOVIC, 2015a)) mostra quatro pontos característicos (P, Q, R, S) em que ocorre o fenômeno do salto. Com o aumento do parâmetro de controle $V_{m}\left(M_{2}\right)$ a frequência do motor aumenta e a amplitude de vibração também aumenta até o ponto $\mathrm{P}$ onde ocorre o salto fazendo o sistema passar para o ponto Q com grande diminuição de amplitude e grande aumento da frequência. No processo inverso quando $V_{m}$ diminui a frequência diminui até o ponto R onde novamente ocorre o salto fazendo o sistema passar para o ponto S (aumento de amplitude e diminuição da frequência). A linha pontilhada no gráfico representa uma região em que o sistema não percorre. 


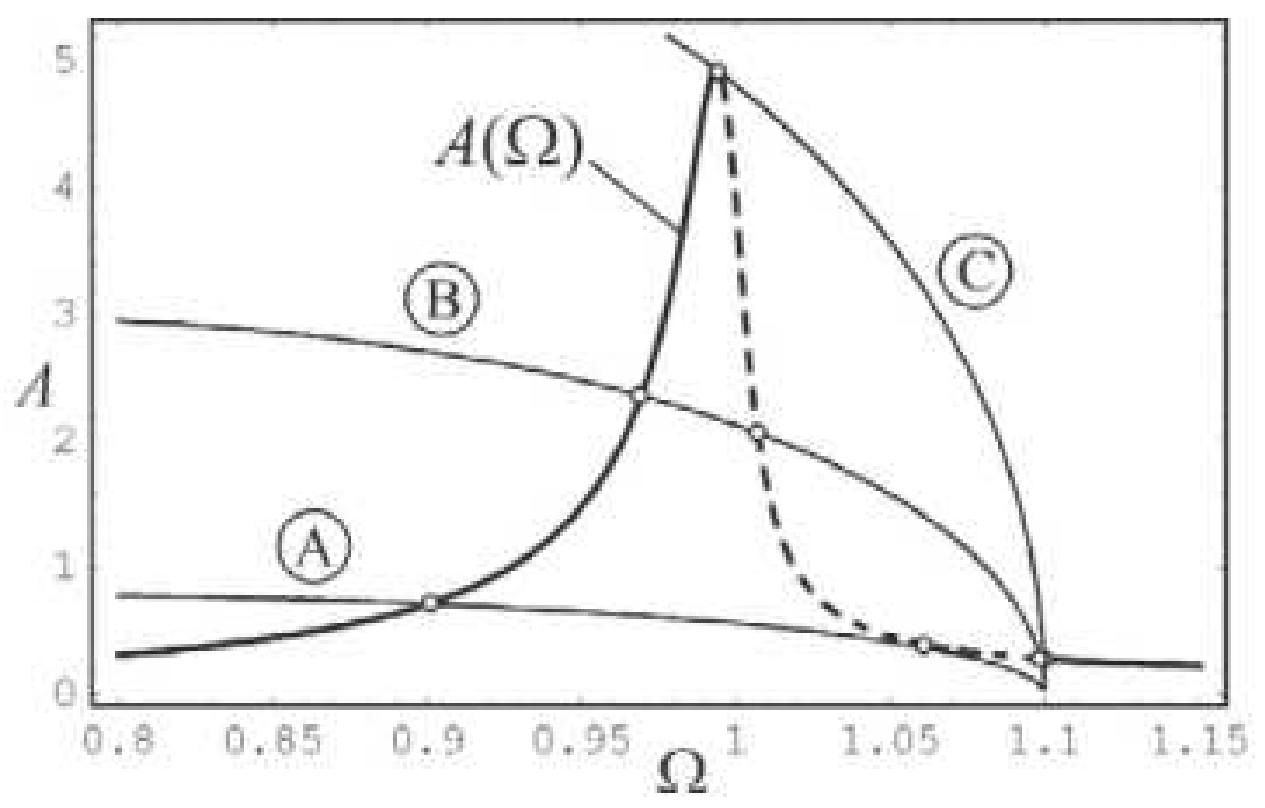

Figura 35 - Curva característica de amplitude e frequência para o sistema e o motor (Adaptado de (ZUKOVIC; CVETICANIN, 2009).

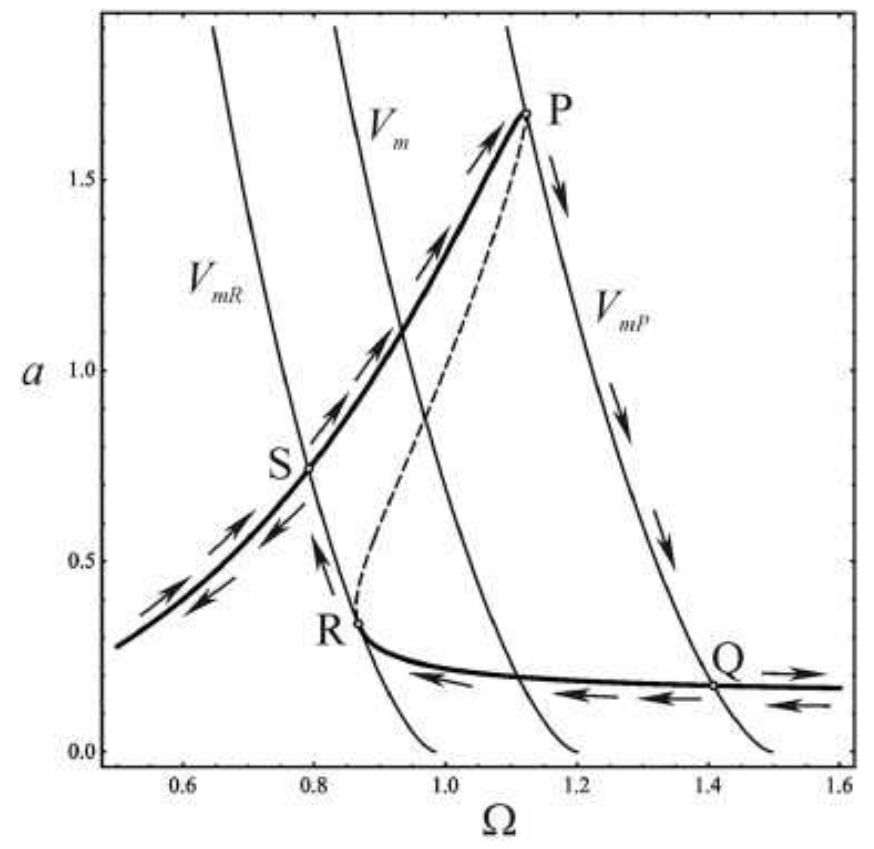

Figura 36 - Pontos característicos na curva de amplitude e frequência (Adaptado de (CVETICANIN; ZUKOVIC, 2015a). 


\subsection{Exemplos numéricos de um sistema mecânico não-ideal}

\subsubsection{Sistema mecânico linear}

Nesta seção é apresentado um exemplo de um motor elétrico conectado com um oscilador mecânico linear. Os parâmetros de simulação adotados são (ver Cveticanin e Zukovic (2015a)): $q_{1}=0.15, q_{2}=0.05, \gamma=0.1$ e $C_{m}=1.0$. Nota-se que os parâmetros $q_{1}=0.15, q_{2}=0.05$ são muito menores que uma unidade definindo, portanto, um valor de $\varepsilon=0.1$. Considera-se uma estrutura linear, $\alpha=1$, com dois valores de rigidez elástica $p^{2}=0.81$ e $p^{2}=1.0$.

A figura 37a apresenta a curva de amplitude versus frequência do sistema mecânico (Eq. 5.51) e do motor elétrico (Eq. 5.52) para $p^{2}=0.81$. Para o motor elétrico são plotadas cinco curvas $\left(M_{2}=0.85 ; M_{2}=0.9155\right.$, curva do tipo A do gráfico $35 ; M_{2}=0.92$, curva do tipo B do gráfico $35 ; M_{2}=0.9273$, curva do tipo C do gráfico $35 ; M_{2}=1.0$ ). Observa-se no gráfico da Figura 37b que representa uma amplificação da Figura 37a para a visualização do fenômeno do salto (efeito Sommerfeld). Com o do aumento do valor do parâmetro de controle, $M_{2}$, a frequência aumenta até a frequência de pico $\Omega_{P^{\prime}}=0.9$ (Eq. (5.55) e após esse ponto o sistema salta do ponto $\mathrm{P}$ para o $\mathrm{Q}$ diminuindo a amplitude e aumentando a frequência (curva para $M_{2}=0.9273$ ). No processo inverso, quando a frequência diminui o salto ocorre entre os pontos $\mathrm{R}$ e $\mathrm{S}$ (curva para $M_{2}=0.9155$ ), (ver Fig. 36).

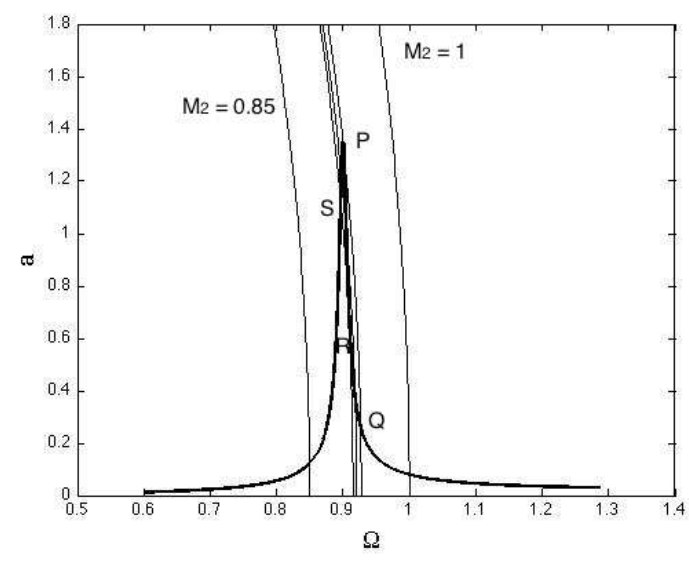

(a)

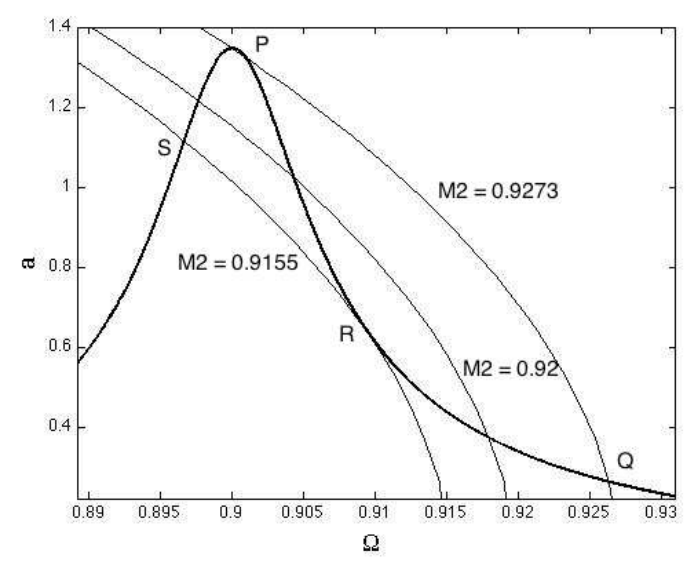

(b)

Figura 37 - Curva característica de amplitude e frequência para o sistema linear e o motor elétrico $\left(\alpha=1, p^{2}=0.81\right)$ : (a) $0.6 \leq \Omega \leq 1.3$; (b) $0.89 \leq \Omega \leq 0.93$ (amplificação).

A figura 38 apresenta a curva de amplitude versus frequência do sistema mecânico e do motor elétrico para $p^{2}=1.0$. As curvas do motor são para os valores: $M_{2}=0.85$, $M_{2}=1.0175, M_{2}=1.03, M_{2}=1.0375$ e $M_{2}=1.2$. Observa-se no gráfico da Figura 38b uma amplificação da Figura 38a em que visualizam-se os pontos P, Q, R, e S referente ao efeito Sommerfeld. Com o do aumento do valor do parâmetro de controle, $M_{2}$, a frequência 
aumenta até a frequência de pico $\Omega_{P^{\prime}}=1.0$ (Eq. (5.55) e após esse ponto o sistema salta do ponto $\mathrm{P}$ para o $\mathrm{Q}$ diminuindo amplitude e aumentando a frequência (curva para $\left.M_{2}=1.0375\right)$. No processo inverso, quando a frequência diminui o salto ocorre entre os pontos R e S (curva para $M_{2}=1.0175$ ), (ver Fig. 36).

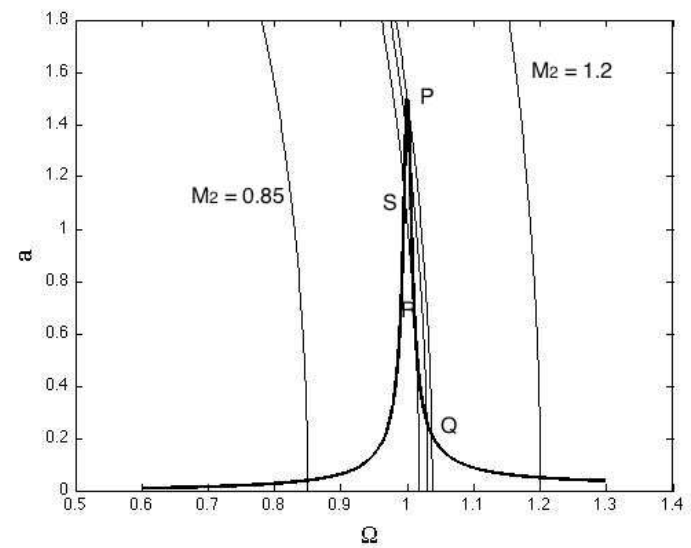

(a)

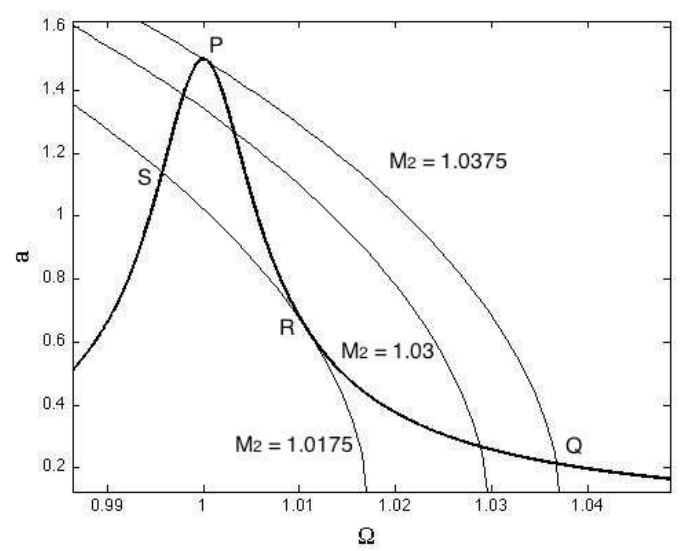

(b)

Figura 38 - Curva característica de amplitude e frequência para o sistema linear e o motor elétrico $\left(\alpha=1, p^{2}=1.0\right)$ : (a) $0.6 \leq \Omega \leq 1.3$; (b) $0.99 \leq \Omega \leq 1.04$ (amplificação).

A seguir apresenta-se as curvas em regime e para o estado não-estacionário de movimento do sistema com $p^{2}=0.81$. Os gráficos das figuras 39, 40 e 41 foram obtidos numericamente resolvendo o seguinte sistema de equações diferenciais:

$$
\begin{array}{r}
z^{\prime \prime}+\varepsilon \gamma z^{\prime}+f_{1}(z)=\varepsilon q_{1}\left(\phi^{\prime \prime} \sin \phi+\phi^{\prime 2} \cos \phi\right) \\
\phi^{\prime \prime}=\varepsilon^{2}\left(q_{2} z^{\prime \prime} \sin \phi+M_{2}-C_{m} \phi^{\prime}\right)
\end{array}
$$

onde a força $f_{1}(z)$ é calculada pela Eq. (5.15) e as condições iniciais são dadas por (5.17). O intervalo de tempo de simulação foi $0 \leq \tau \leq 5000$. A figura 39 mostra o histórico no tempo para o parâmetro de controle do motor $M_{2}=0.92$. As condições iniciais utilizadas foram: $z(0)=0, z^{\prime}(0)=0, \phi(0)=0$ e $\phi^{\prime}(0)=0$. Com essas condições iniciais obtém-se um ponto estável do gráfico 37 , isto é, $(z, \Omega) \approx(1.2,0.9)$ (ver Fig. 41a).

A figura 40 apresenta, também, o histórico no tempo para o parâmetro de controle do motor $M_{2}=0.92$. Nessa figura as condições iniciais utilizadas foram: $z(0)=0$, $z^{\prime}(0)=0, \phi(0)=0$ e $\phi^{\prime}(0)=M_{2}$, obtendo o segundo ponto estável (ver figura 37), ou seja, $(z, \Omega)=\approx(0.38,0.918)$ (ver Fig. 41b). Dessa forma ambas soluções, aproximada analítica (Eqs. 5.51 e 5.52) e numérica (5.57), apresentam uma boa concordância.

A figura 41 apresenta o histórico no tempo da frequência $(\Omega-\tau)$ para duas condições iniciais distintas: $z(0)=0, z^{\prime}(0)=0, \phi(0)=0$ e $\phi^{\prime}(0)=0.0$ (figura 41a) e $z(0)=0$, $z^{\prime}(0)=0, \phi(0)=0$ e $\phi^{\prime}(0)=M_{2}$ (figura 41b). Observa-se que dependendo se a frequência do motor está aumentando ou diminuindo os valores obtidos no regime permanente são diferentes. 


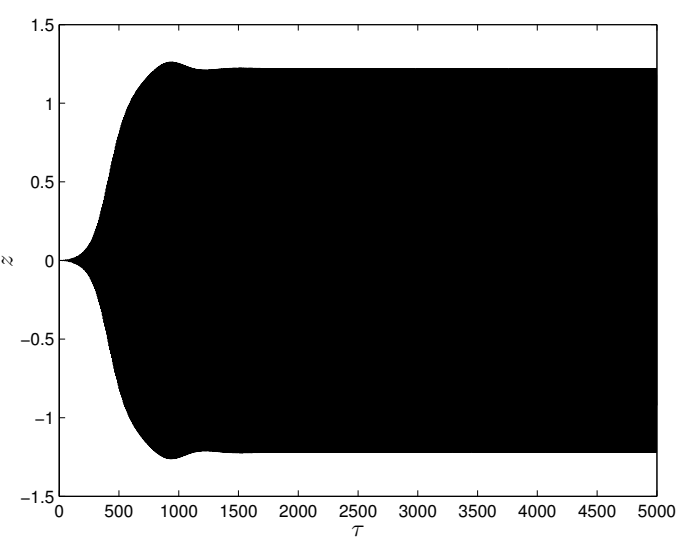

(a)

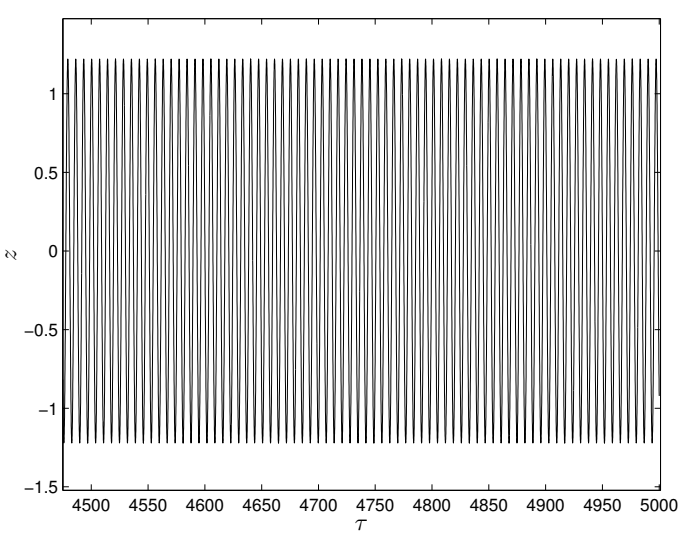

(b)

Figura 39 - Histórico no tempo $z-\tau$ para $\alpha=1, p^{2}=0.81, M_{2}=0.92(z(0)=0$, $z^{\prime}(0)=0, \phi(0)=0$ e $\left.\phi^{\prime}(0)=0.0\right)$ : (a) movimento não-estacionário; (b) regime permanente.

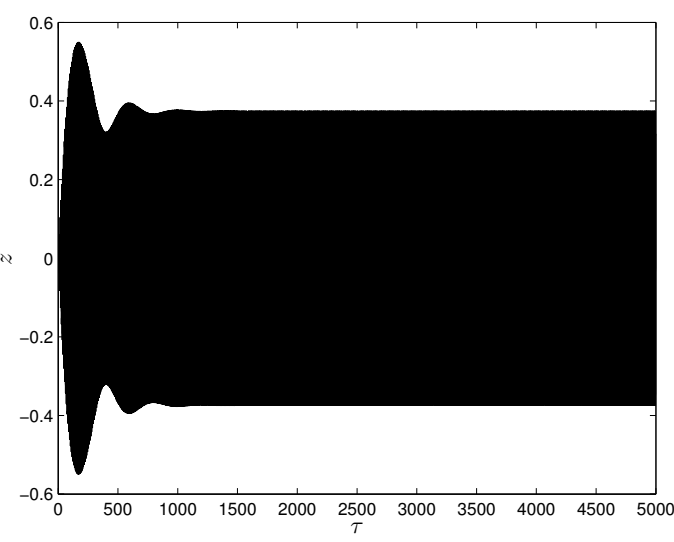

(a)

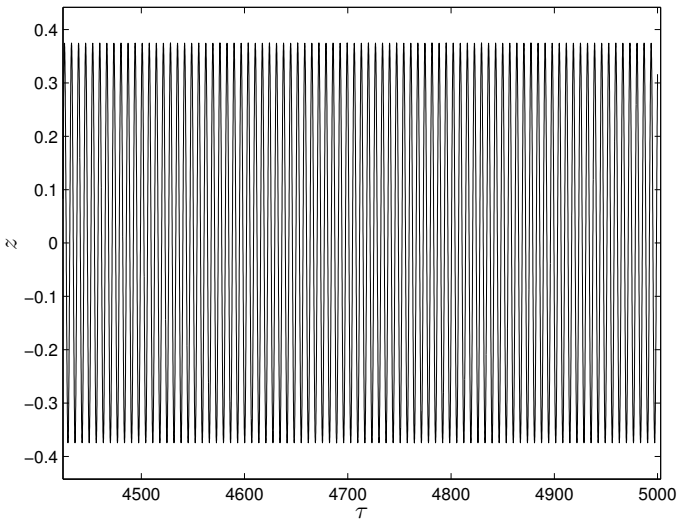

(b)

Figura 40 - Histórico no tempo $z-\tau$ para $\alpha=1, p^{2}=0.81, M_{2}=0.92(z(0)=0$, $z^{\prime}(0)=0, \phi(0)=0$ e $\phi^{\prime}(0)=M_{2}$ ): (a) movimento não-estacionário; (b) regime permanente.

A seguir analisa-se o sistema mecânico impactando uma parede sólida com rigidez $p_{I}^{2}=10000$. A figura 42 apresenta o plano de fase para o parâmetro de controle $M_{2}=0.92$ considerando o regime permanente, isto é, a partir de $80 \%$ do tempo transcorrido. $\mathrm{Na}$ figura 42a são utilizadas as condições iniciais $z(0)=0, z^{\prime}(0)=0, \phi(0)=0$ e $\phi^{\prime}(0)=0.0$ e a parede está localizada na posição $z^{*}=0.8$ e na figura $42 \mathrm{~b}$ as condições iniciais são $z(0)=0$, $z^{\prime}(0)=0, \phi(0)=0$ e $\phi^{\prime}(0)=M_{2}$ com a parede localizada em $z^{*}=0.35$. Observa-se em ambos os casos que a parede limita o movimento à direita do bloco afetando também o movimento à esquerda pela inércia do movimento. 


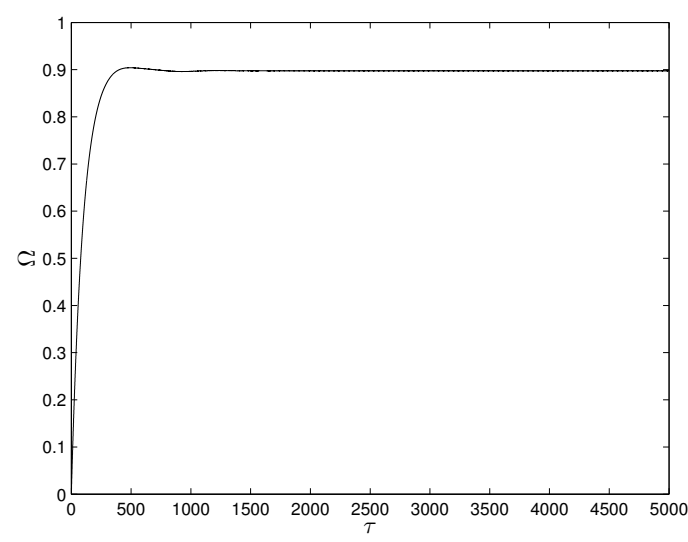

(a)

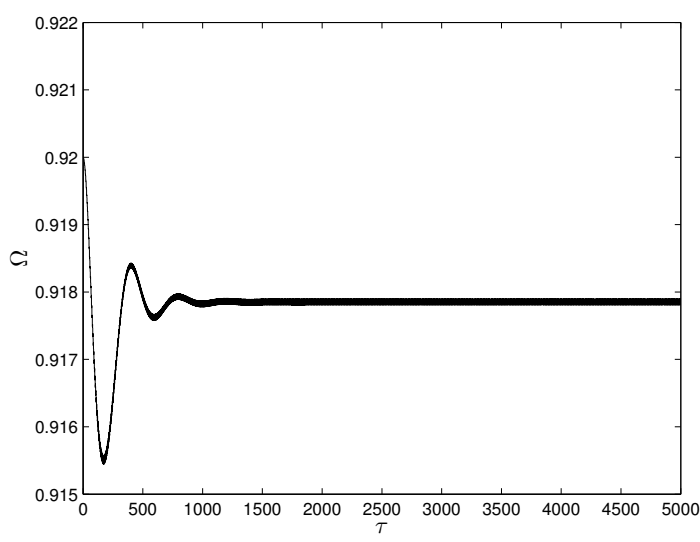

(b)

Figura 41 - Histórico no tempo $\Omega-\tau$ para $\alpha=1, p^{2}=0.81, M_{2}=0.92$ : (a) $z(0)=0$, $z^{\prime}(0)=0, \phi(0)=0$ e $\phi^{\prime}(0)=0.0 ;(\mathrm{b}) z(0)=0, z^{\prime}(0)=0, \phi(0)=0 \mathrm{e}$ $\phi^{\prime}(0)=M_{2}$.

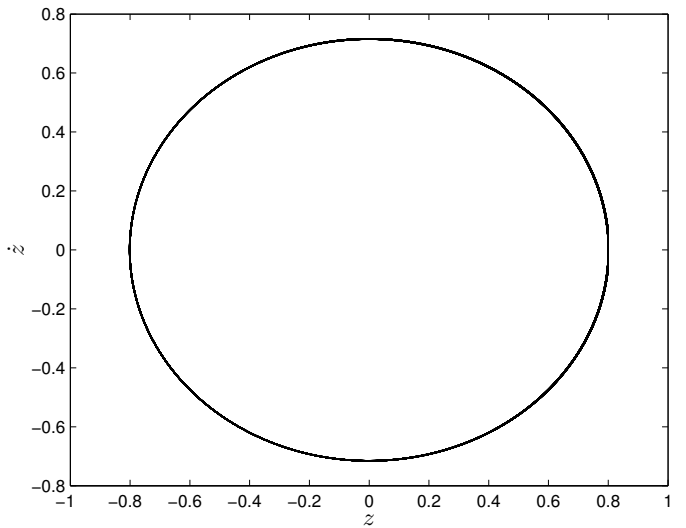

(a)

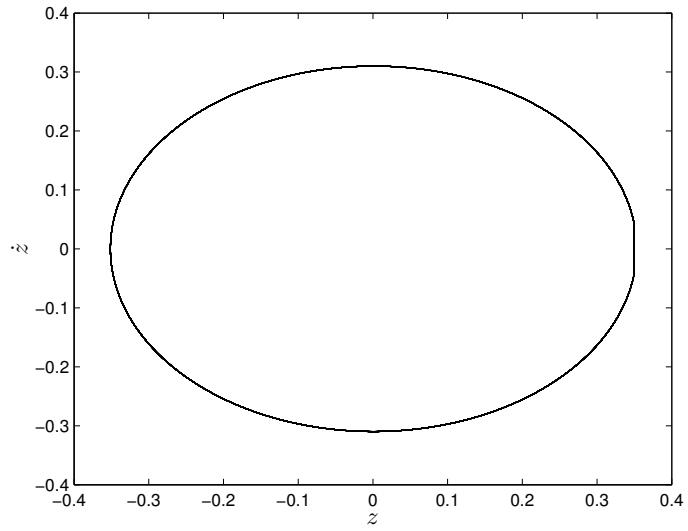

(b)

Figura $42-$ Plano de fase $z-z^{\prime}$ para $\alpha=1, p^{2}=0.81, M_{2}=0.92$ : (a) $z^{*}=0.8, z(0)=0$, $z^{\prime}(0)=0, \phi(0)=0$ e $\phi^{\prime}(0)=0.0 ;(\mathrm{b}) z^{*}=0.35, z(0)=0, z^{\prime}(0)=0, \phi(0)=0$ e $\phi^{\prime}(0)=M_{2}$. 


\subsubsection{Sistema mecânico não-linear}

Nesta seção é apresentado um exemplo de um motor elétrico conectado com um oscilador mecânico não-linear. Existem diversos materiais com propriedades não-lineares. Jutte (2008) propõe uma metodologia para o estudo de molas não-lineares. Na presente seção serão analisados três tipos de materiais elásticos através da variação do expoente $\alpha$, isto é, $\alpha=4 / 3 \operatorname{com} p^{2}=0.81, \alpha=5 / 3 \operatorname{com} p^{2}=0.81$ e $\alpha=2 / 3 \operatorname{com} p^{2}=0.36$. Os outros parâmetros adotados nas simulações são semelhantes aos da seção anterior, isto é, $C_{m}=1.0, q_{1}=0.15, q_{2}=0.05$ e $\gamma=0.1$, onde nota-se que $q_{1} \ll 1, q_{2} \ll 1$ e $\gamma \ll 1$.

A figura 43 apresenta a curva de amplitude versus frequência do sistema mecânico (Eq. 5.51) e do motor elétrico (Eq. 5.52) para $\alpha=4 / 3$ e $p^{2}=0.81$. A frequência constante que é função da ordem de não-linearidade $\alpha$ é dada por: $\omega_{4 / 3}{ }^{2}=0.93927$ (ver Eq. 5.49). As curvas de amplitude-frequência do motor elétrico são plotadas para diversos valores do parâmetro de controle: $M_{2}=1.1, M_{2}=M_{2 P^{\prime}}=0.9497$ (curva do salto entre os pontos P e Q), $M_{2}=0.8$ (curva intermediária) e $M_{2}=0.6302$ (curva do salto entre os pontos R e S) e $M_{2}=0.45$. Observa-se no gráfico da Figura 43 o fenômeno do salto (efeito Sommerfeld). Com o aumento do valor do parâmetro de controle, $M_{2}$, a frequência aumenta até a frequência do ponto P onde ocorre o salto, isto é, diminuição da amplitude e aumento da frequência (ponto Q). Partindo de um alto torque do motor e diminuindo a voltagem, a frequência diminui até o ponto $\mathrm{R}$ onde ocorre o salto em que o sistema estabiliza no regime permanente no ponto S com um pequeno aumento da amplitude e pequena diminuição na frequência.

A figura 44 apresenta a curva de amplitude versus frequência do sistema mecânico (Eq. 5.51) e do motor elétrico (Eq. 5.52) para $\alpha=5 / 3$ e $p^{2}=0.81$. A frequência constante que é função da ordem de não-linearidade $\alpha$ é dada por: $\omega_{5 / 3}^{2}=0.88513$ (ver Eq. 5.49). As curvas de amplitude-frequência do motor elétrico são plotadas para diversos valores do parâmetro de controle: $M_{2}=1.15, M_{2}=M_{2 P^{\prime}}=0.9868$ (curva do salto entre os pontos $\mathrm{P}$ e Q), $M_{2}=0.7$ (curva intermediária), $M_{2}=0.3655$ (curva do salto entre os pontos R e S) e $M_{2}=0.25$. Observa-se, também, no gráfico da Figura 44 o fenômeno do salto (efeito Sommerfeld). Como no caso do gráfico da Figura 43, com o aumento do valor do parâmetro de controle, $M_{2}$, a frequência aumenta até a frequência do ponto $\mathrm{P}$ onde ocorre o salto, isto é, diminuição da amplitude e aumento da frequência (ponto Q). Partindo de um alto torque do motor e diminuindo a voltagem, a frequência diminui até o ponto $\mathrm{R}$ onde ocorre o salto em que o sistema estabiliza no regime permanente no ponto S com um pequeno aumento da amplitude e pequena diminuição na frequência (ver Fig. 44b).

A seguir resolve-se numericamente o sistema de equações diferenciais (5.57) para as ordens de não-linearidades $\alpha=4 / 3$ e $\alpha=5 / 3$ no intervalo de tempo adimensional $0 \leq \tau \leq 5000$. A figura 45 apresenta simulações para as condições iniciais $z(0)=0$, $z^{\prime}(0)=0, \phi(0)=0$ e $\phi^{\prime}(0)=0$ e não-linearidade $\alpha=4 / 3$ com $p^{2}=0.81$ equivalente ao 


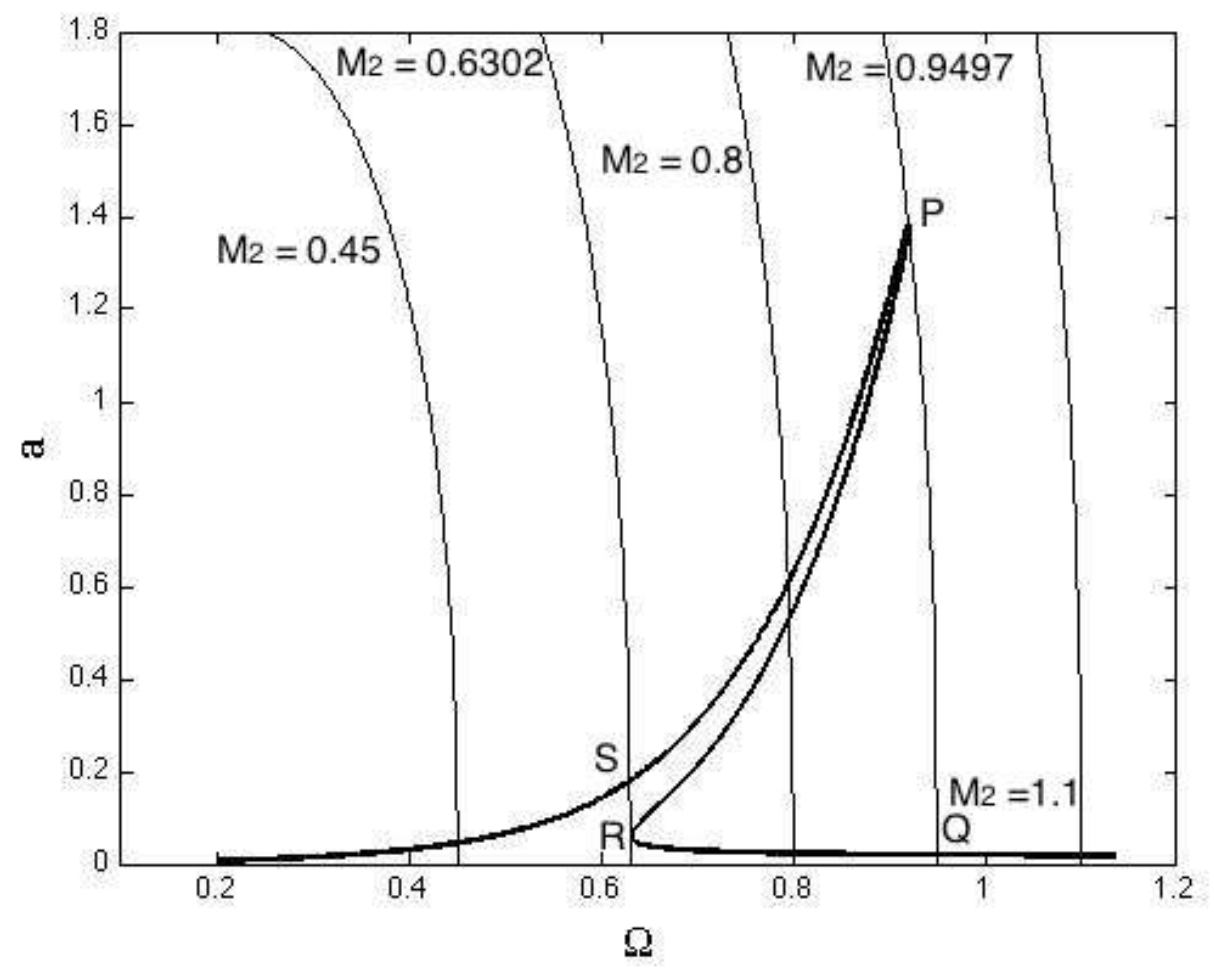

Figura 43 - Curva característica de amplitude e frequência para o sistema não-linear e o motor elétrico $\left(\alpha=4 / 3\right.$ e $\left.p^{2}=0.81\right)$.

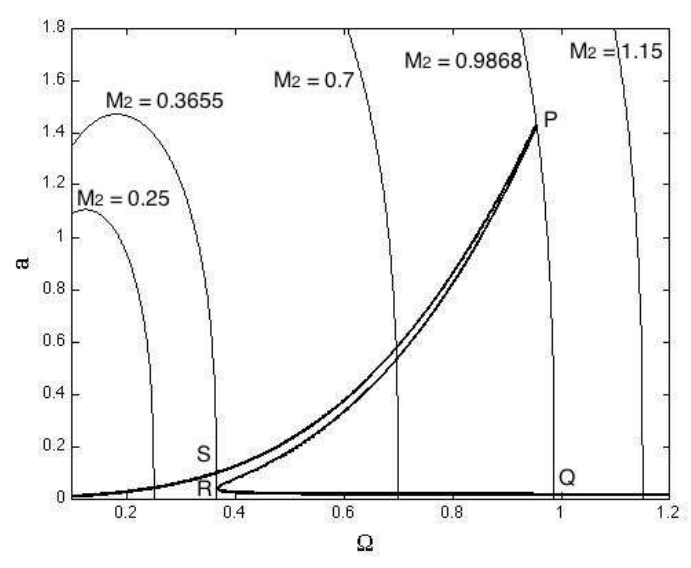

(a)

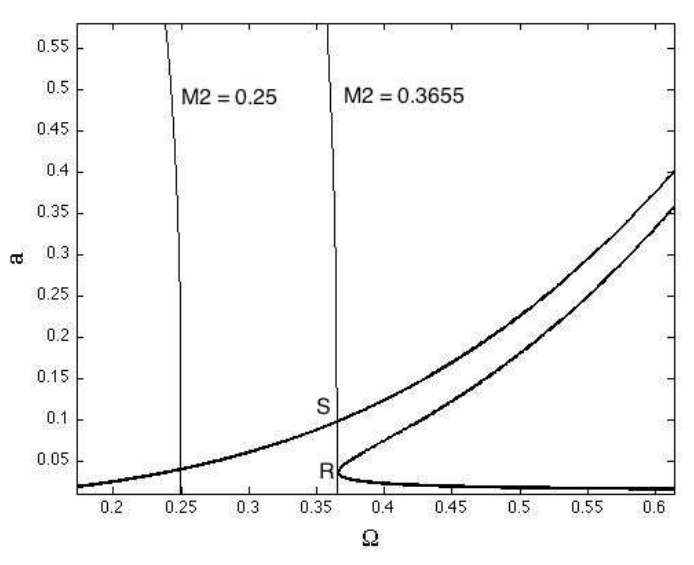

(b)

Figura 44 - Curva característica de amplitude e frequência para o sistema não-linear e o motor elétrico $\left(\alpha=5 / 3\right.$ e $p^{2}=0.81$ ): (a) $0 \leq \Omega \leq 1.2$; (b) $0.2 \leq \Omega \leq 0.6$ (amplificação). 
gráfico 43 para $M_{2}=0.8$. A figura 45 a mostra o histórico no tempo para a posição $z$ que entra em regime permanente em $\tau \approx 1000$ com uma amplitude $a \approx 0.611$. A figura 45b mostra o histórico no tempo para a frequência $\Omega$ que entra em regime permanente em $\tau \approx 2000$ com uma frequência $\Omega \approx 0.795$. Esses valores estão em concordância com os ilustrados na intersecção das curvas de amplitude do sistema e torque do motor (Fig. 43) para $M_{2}=0.8$.

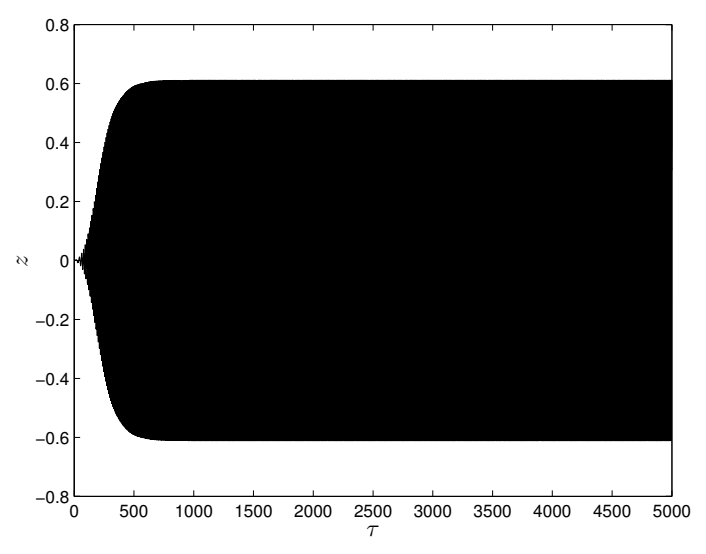

(a)

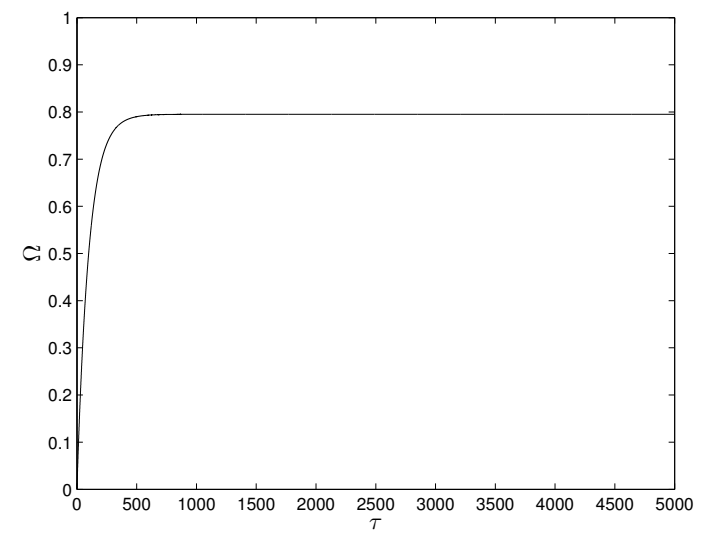

(b)

Figura 45 - Histórico no tempo para $\alpha=4 / 3, M_{2}=0.8, p^{2}=0.81,\left(z(0)=0, z^{\prime}(0)=0\right.$, $\phi(0)=0$ e $\left.\phi^{\prime}(0)=0.0\right)$ : (a) $z-\tau ;$ (b) $\Omega-\tau$.

A figura 46 apresenta simulações para as condições iniciais $z(0)=0, z^{\prime}(0)=0$, $\phi(0)=0$ e $\phi^{\prime}(0)=1$ e não-linearidade $\alpha=4 / 3$ com $p^{2}=0.81$ (ver gráfico 43 para $M_{2}=0.8$ ) em que se obtém a segunda solução estável em regime permanente quando $\tau \approx 1000$. A figura 46a mostra o histórico no tempo para a posição $z$ que entra em regime permanente em $\tau \approx 1000$ com uma amplitude $a \approx 0.0227$. A figura 46b mostra o histórico no tempo para a frequência $\Omega$ em que a frequência converge para $\Omega=0.8$. Esses valores também estão em concordância com os ilustrados na intersecção das curvas de amplitude do sistema e torque do motor (Fig. 43) para $M_{2}=0.8$.

A figura 47 apresenta simulações para as condições iniciais $z(0)=0, z^{\prime}(0)=0$, $\phi(0)=0$ e $\phi^{\prime}(0)=0$ e não-linearidade $\alpha=5 / 3 \operatorname{com} p^{2}=0.81$ equivalente ao gráfico 44 para $M_{2}=0.7$. A figura 47 a mostra o histórico no tempo para a posição $z$ que entra em regime permanente em $\tau \approx 1000$ com uma amplitude $a \approx 0.575$. A figura $47 \mathrm{~b}$ mostra $\mathrm{o}$ histórico no tempo para a frequência $\Omega$ que entra em regime permanente em $\tau \approx 1000$ com uma frequência $\Omega \approx 0.696$. Esses valores estão em concordância com os ilustrados na intersecção das curvas de amplitude do sistema e torque do motor (Fig. 44a) para $M_{2}=0.7$.

A figura 48 apresenta simulações para as condições iniciais $z(0)=0, z^{\prime}(0)=0$, $\phi(0)=0$ e $\phi^{\prime}(0)=1$ e não-linearidade $\alpha=5 / 3$ com $p^{2}=0.81$ (ver gráfico 44 para $\left.M_{2}=0.7\right)$ em que se obtém a segunda solução estável em regime permanente quando 


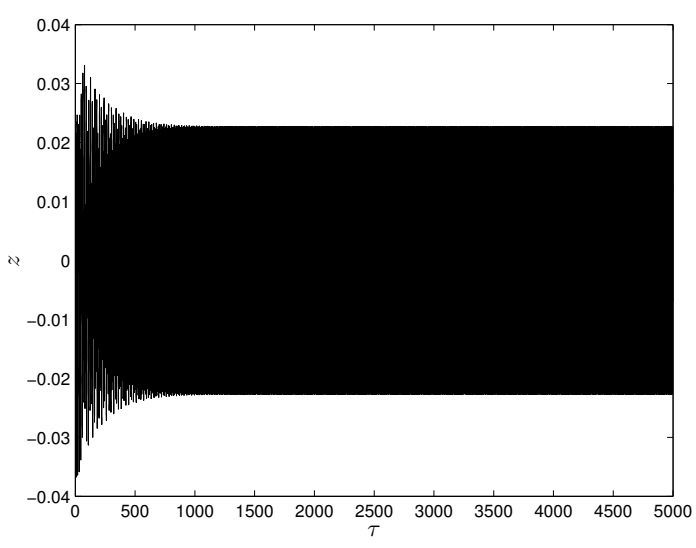

(a)

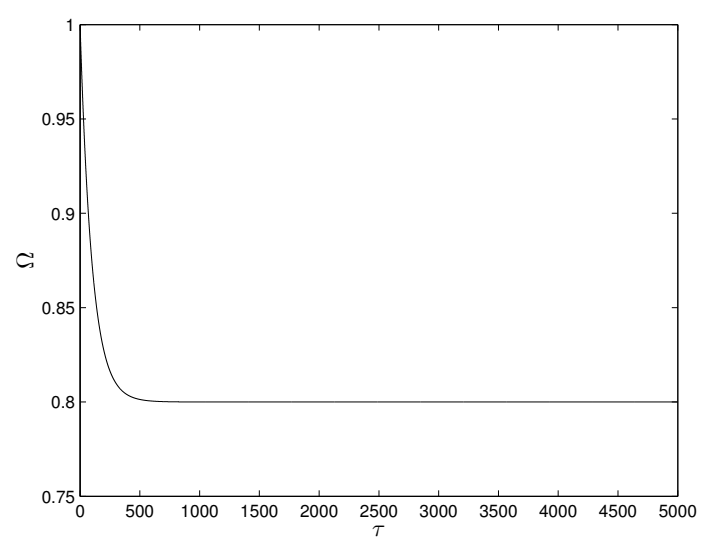

(b)

Figura 46 - Histórico no tempo para $\alpha=4 / 3, M_{2}=0.8, p^{2}=0.81,\left(z(0)=0, z^{\prime}(0)=0\right.$, $\phi(0)=0$ e $\left.\phi^{\prime}(0)=1.0\right)$ : (a) $z-\tau$; (b) $\Omega-\tau$.

$\tau \approx 1000$. A figura 48a mostra o histórico no tempo para a posição $z$ que entra em regime permanente em $\tau \approx 1000$ com uma amplitude $a \approx 0.0165$. A figura 48b mostra o histórico no tempo para a frequência $\Omega$ em que a frequência converte para $\Omega=0.7$. Esses valores também estão em concordância com os ilustrados na intersecção das curvas de amplitude do sistema e torque do motor (Fig. 44a) para $M_{2}=0.7$.

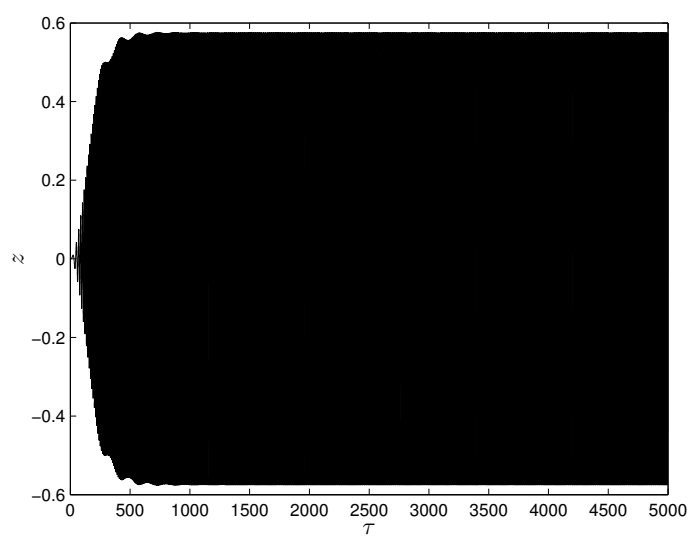

(a)

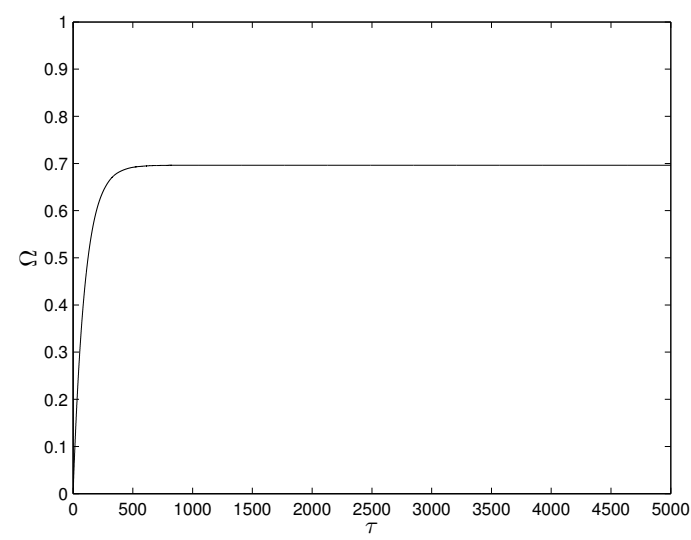

(b)

Figura 47 - Histórico no tempo para $\alpha=5 / 3, M_{2}=0.7, p^{2}=0.81,\left(z(0)=0, z^{\prime}(0)=0\right.$, $\phi(0)=0$ e $\left.\phi^{\prime}(0)=0.0\right)$ : (a) $z-\tau$; (b) $\Omega-\tau$.

A figura 49 apresenta a curva de amplitude versus frequência do sistema mecânico (Eq. 5.51) e do motor elétrico (Eq. 5.52) para $\alpha=2 / 3$ e $p^{2}=0.36$. Nesse caso, como $\alpha<1$ o gráfico de amplitude do sistema tem uma inclinação para o lado esquerdo, contrário ao caso das figuras 43 e 44, sendo o valor da frequência é $\omega_{2 / 3}^{2}=1.06844$ (ver Eq. 5.49). As curvas de amplitude-frequência do motor elétrico são plotadas para diversos valores do parâmetro de controle: $M_{2}=1.2, M_{2}=M_{2 P^{\prime}}=0.9085$ (curva do salto entre os pontos $\mathrm{P}$ e Q), $M_{2}=0.8$ (curva intermediária), $M_{2}=M_{2 R^{\prime}}=0.6359$ (curva do salto entre os 


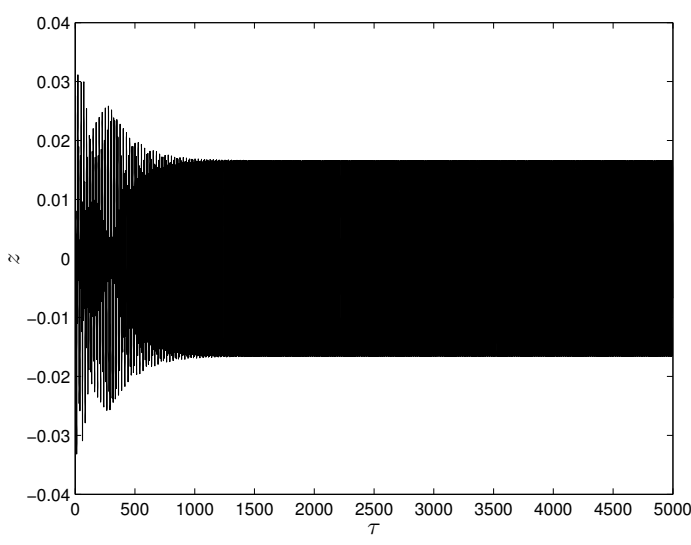

(a)

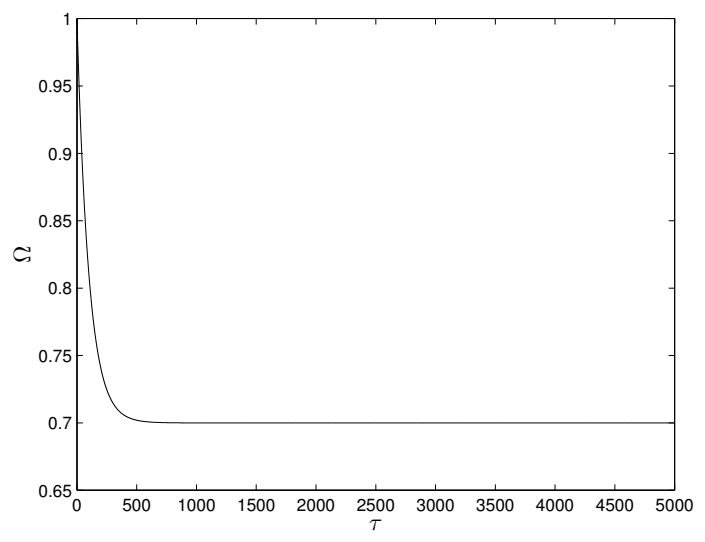

(b)

Figura 48 - Histórico no tempo para $\alpha=5 / 3, M_{2}=0.7, p^{2}=0.81,\left(z(0)=0, z^{\prime}(0)=0\right.$, $\phi(0)=0$ e $\left.\phi^{\prime}(0)=1.0\right)$ : (a) $z-\tau$; (b) $\Omega-\tau$.

pontos R e S) e $M_{2}=0.45$ (curva com um ponto estável). Como no caso da figuras 43 e 44 o fenômeno do salto (efeito Sommerfeld) ocorre na figura 49 entre os pontos P e Q (aumentando a frequência) e entre $\mathrm{R}$ e $\mathrm{S}$ (diminuindo a frequência). Caso fosse escolhido o valor de $p^{2}=0.81$ (rigidez maior) a curva de amplitude do sistema se deslocaria para a direita e o efeito Sommerfeld poderia não aparecer se não fosse aumentado o torque do motor.

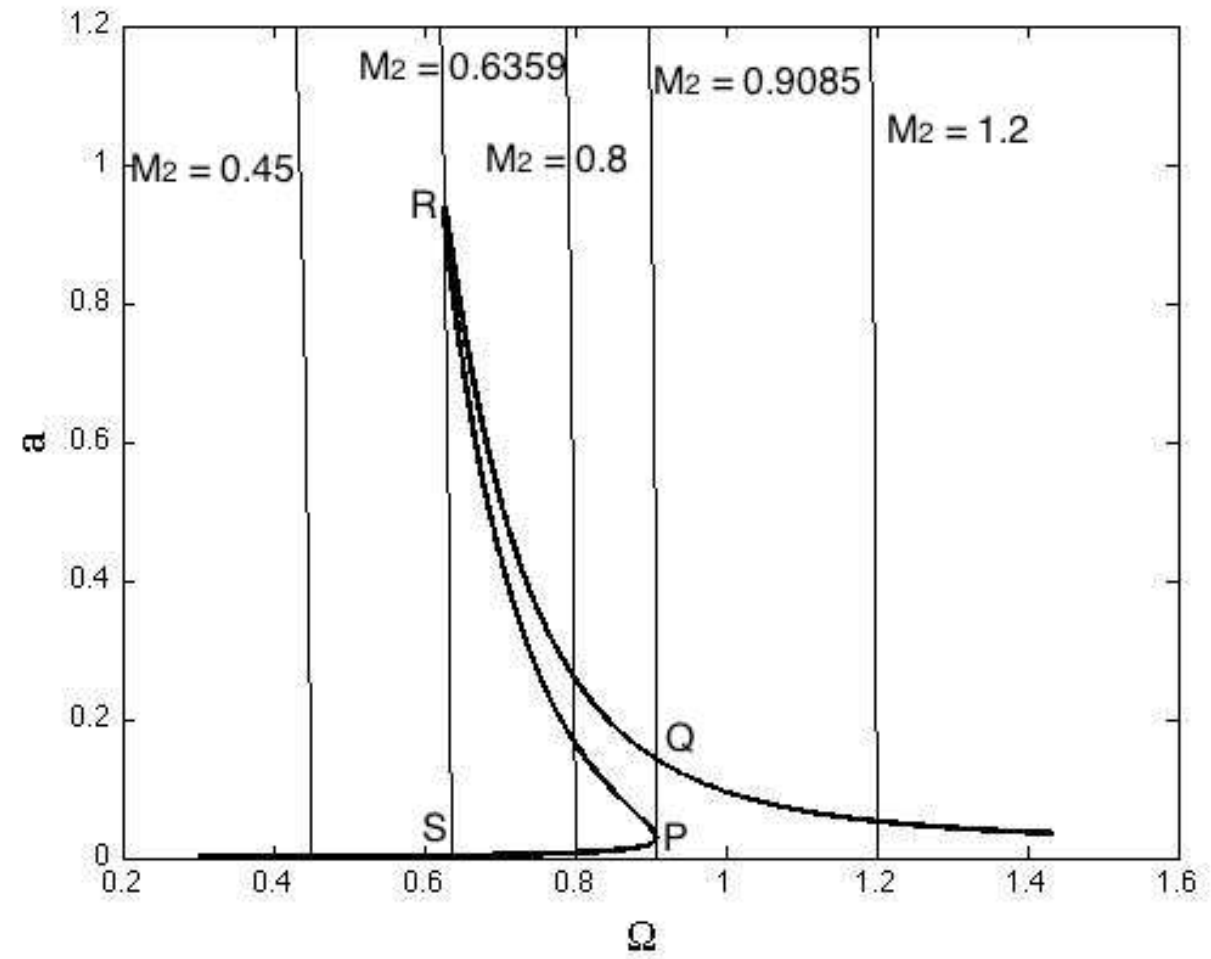

Figura 49 - Curva característica de amplitude e frequência para o sistema não-linear e o motor elétrico $\left(\alpha=2 / 3\right.$ e $\left.p^{2}=0.36\right)$. 
A seguir analisa-se o sistema mecânico com não-linearidade impactando uma parede sólida com rigidez $p_{I}^{2}=10000$. As figuras 50 e 51 apresentam o histórico no tempo e o plano de fase para a ordem de não-linearidade $\alpha=4 / 3$, a rigidez do sistema $p^{2}=0.81$ e o parâmetro de controle $M_{2}=0.8$. Os gráficos da Figura 50 são para as condições iniciais $z(0)=0, z^{\prime}(0)=0, \phi(0)=0$ e $\phi^{\prime}(0)=0.0$ e a posição da parede $z^{*}=0.4$, enquanto, os gráficos da Figura 51 são para as condições iniciais $z(0)=0, z^{\prime}(0)=0, \phi(0)=0$ e $\phi^{\prime}(0)=1.0$ e a posição da parede $z^{*}=0.018$. O gráfico da Figura 50a entra em regime no instante aproximado de $\tau=1000$ limitando a amplitude de movimento do bloco em $a \approx 0.4$ que é menor do que o movimento sem a parede $\approx 0.6$ (ver Fig. $45 \mathrm{a}$ ). O gráfico da figura 51a mostra a posição $z$ que entra em regime permanente em $\tau \approx 2000$. Observa-se no gráfico que além de limitar o movimento à direita do bloco $\left(z^{*} \approx 0.018\right)$ o movimento à esquerda é amplificado passando no regime de $\approx-0.02$ para o valor de $\approx-0.06$ devido à não-linearidade da mola do sistema mecânico. Os limites de posição e velocidade são observados nos planos de fase das figuras 50b e 51b.

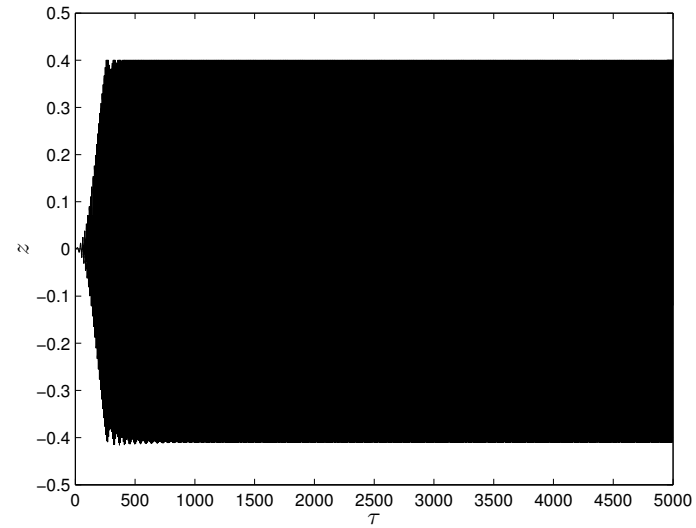

(a)

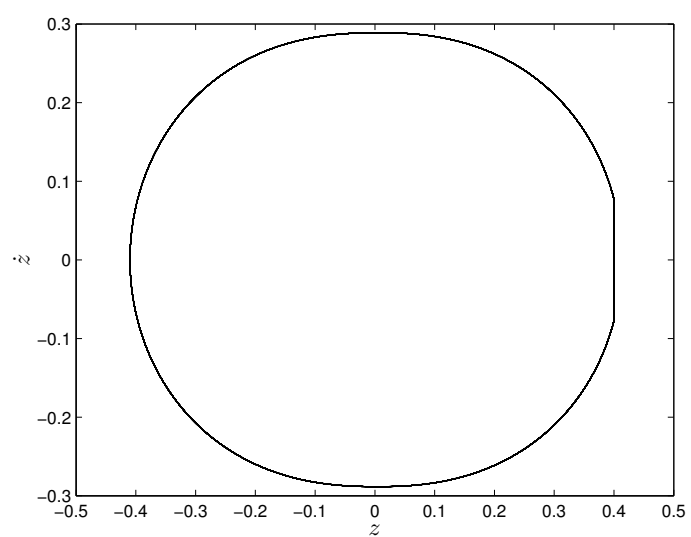

(b)

Figura 50 - Diagramas de impacto, $\alpha=4 / 3, p^{2}=0.81, M_{2}=0.8, z^{*}=0.4, z(0)=0$, $z^{\prime}(0)=0, \phi(0)=0$ e $\phi^{\prime}(0)=0.0$ : (a) Histórico no tempo $z-\tau$; (b) Plano de fase $z-z^{\prime}$. 


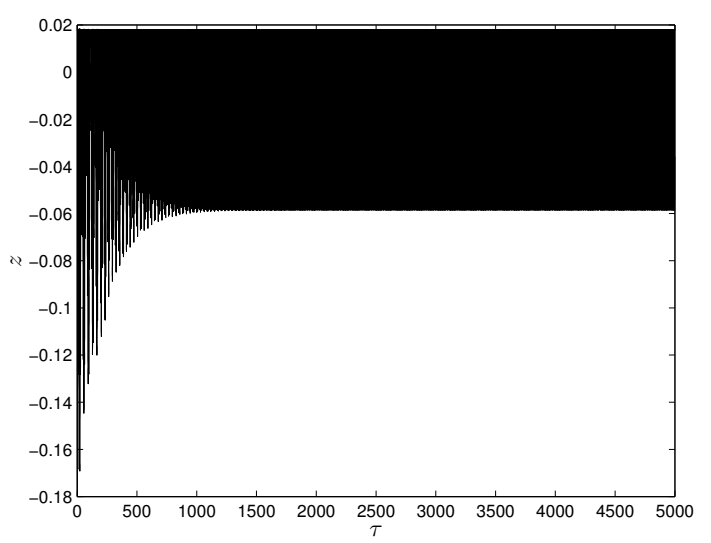

(a)

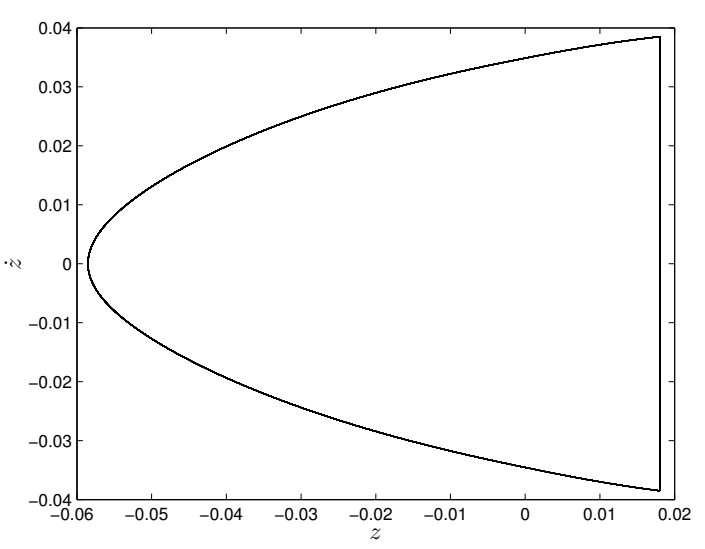

(b)

Figura 51 - Diagramas de impacto, $\alpha=4 / 3, p^{2}=0.81, M_{2}=0.8, z^{*}=0.018, z(0)=0$, $z^{\prime}(0)=0, \phi(0)=0$ e $\phi^{\prime}(0)=1.0$ : (a) Histórico no tempo $z-\tau$; (b) Plano de fase $z-z^{\prime}$. 


\subsubsection{Sistema mecânico colidindo em um parede com forças dissipativas}

Nesta seção analisa-se o sistema mecânico linear $(\alpha=1)$ considerando a colisão normal com a parede simulada com forças conservativas (Eq. 4.36) e dissipativas (Eq. 4.47), (ver (NAVARRO; BALTHAZAR; BRASIL, 2014)). As equações de movimento do sistema mecânico acoplado ao motor elétrico são:

$$
\begin{array}{r}
(M+m) \ddot{x}+c \dot{x}+k x+F_{I}=m d\left(\ddot{\phi} \sin (\phi)+\dot{\phi}^{2} \cos (\phi)\right) \\
\left(J+m d^{2}\right) \ddot{\phi}=m d \ddot{x} \sin (\phi)+V_{m}{ }^{*}-C_{m}{ }^{*} \dot{\phi}
\end{array}
$$

onde a força de impacto é dada por $\left(c_{I} \gg c\right.$ e $\left.k_{I} \gg k\right)$ :

$$
\begin{aligned}
& F_{I} \approx c_{I} x^{1 / 4} \dot{x}+k_{I} x^{3 / 2}, \quad \text { se } \quad x>G>0 \\
& F_{I}=0, \quad \text { se } \quad x \leq G
\end{aligned}
$$

onde $k_{I}$ é a rigidez não-linear (Eq. 4.38) e $c_{I}$ é o coeficiente de amortecimento não-linear (Eq. 4.49). As condições inciais são dadas por:

$$
x(0)=x_{0}, \quad \dot{x}(0)=0, \quad \phi(0)=0, \quad \dot{\phi}(0)=\omega_{0} .
$$

Os parâmetros do sistema mecânico utilizado nas simulações estão listados na Tabela 18. O motor elétrico usado é um de corrente contínua da Maxon ${ }^{\circledR}$ de $200 \mathrm{~W}$ modelo RE50 com $50 \mathrm{~mm}$ de diâmetro. A massa desbalanceada do rotor $m$ faz parte do motor e equivale a $20 \%$ da massa do motor. A massa do conjunto $M$ inclui a massa do bloco e a a massa do motor sem a massa desbalanceada, isto é, $M=M_{b}+M_{m}-m=3.88 \mathrm{~kg}$ e, portanto, $M+m=4.1 \mathrm{~kg}$. Nesta seção o momento de inércia de massa das partes rotativas será o do próprio rotor, isto é, $J=J_{m}$. Utilizando os dados da Tabela 18 determinam-se os parâmetros $\Omega_{0}=623.3 \mathrm{rad} / \mathrm{s} \approx 5950 \mathrm{rpm}, M_{0}=8.971 \mathrm{Nm}, \omega^{*}=8.55 \mathrm{rad} / \mathrm{s}, C_{m}{ }^{*}=0.01439 \mathrm{Nms}$, $\gamma^{*}=3.91 \times 10^{-4}, q_{1}^{*}=0.01363$ e $q_{2}^{*}=1.5682$. O parâmetro $q_{2}^{*}>1$ e, portanto, as simplificações anteriores não são válidas. Devido a complexidade de se derivar soluções analíticas para o sistema de equações, será utilizado o método numérico de Runge-Kutta para a obtenção das soluções numericamente.

As equações (5.58) são resolvidas numericamente com as condições iniciais (5.60). Inicialmente analisa-se esse sistema sem considerar o impacto, isto é, considerando o valor de $G$ muito alto. O parâmetro de controle utilizado é a voltagem $V$ de entrada do motor elétrico. O tempo total de simulação considerado é de $t=10$ min sendo que o regime permanente foi alcançado nos últimos 2 min de simulação. As simulações são feitas para duas voltagens do motor elétrico, isto é, $V=0.336 \mathrm{~V}\left(V_{m}{ }^{*}=0.1256 \mathrm{Nm}\right)$ e $V=24.0 \mathrm{~V}\left(V_{m}{ }^{*}=8.971 \mathrm{Nm}\right)$. As condições iniciais utilizadas foram: $x(0)=0 \mathrm{~m}$, $\dot{x}(0)=0 \mathrm{~m} / \mathrm{s}, \phi(0)=0 \mathrm{rad}, \dot{\phi}(0)=0 \mathrm{rad} / \mathrm{s}$. As figuras 52 e 53 mostram o plano de fase $\phi-\dot{\phi}$ e os históricos no tempo $\phi-t, \dot{\phi}-t$, respectivamente, para as voltagens $V=0.336 \mathrm{~V}$ e $V=24.0 \mathrm{~V}$. A Figura 52 mostra que, em regime permanente, o bloco alcança uma 
Tabela 18 - Parâmetros do sistema mecânico para a simulação numérica.

\begin{tabular}{ll}
\hline Massa do motor & $M_{m}=1.1 \mathrm{~kg}$ \\
Massa desbalanceada & $m=0.22 \mathrm{~kg}$ \\
Massa do bloco sem o motor & $M_{b}=3 \mathrm{~kg}$ \\
Excentricidade & $d=0.0127 \mathrm{~m}$ \\
Momento de inércia de massa do motor & $J_{m}=5.36 \times 10^{-5} \mathrm{kgm}^{2}$ \\
Coeficiente de rigidez da mola & $k=300 \mathrm{~N} / \mathrm{m}$ \\
Coeficiente de amortecimento & $c=1 \mathrm{Ns} / \mathrm{m}$ \\
Voltagem nominal do motor & $V_{0}=24 \mathrm{~V}$ \\
Constante de torque & $K_{T}=0.0385 \mathrm{Nm} / \mathrm{A}$ \\
Constante de voltagem & $K_{E}=0.0385 \mathrm{~V} / \mathrm{rads}^{-1}$ \\
Resistência elétrica & $R=0.103 \Omega$ \\
Comprimento não deformado da mola & $l_{0}=0.05 \mathrm{~m}$ \\
\hline
\end{tabular}

amplitude de deslocamento de $z=0.0238 \mathrm{~m}$ (por exemplo no instante $t=598.9 \mathrm{~s}$ ) e uma velocidade angular média do motor de $\dot{\phi}=8.566 \mathrm{rad} / \mathrm{s}$. Na Figura $53(V=24.0 \mathrm{~V})$ observa-se, no regime permanente, que bloco alcança uma amplitude de deslocamento menor $\left(z=6.816 \times 10^{-4} \mathrm{~m}\right.$, por exemplo no instante $\left.t=553 \mathrm{~s}\right)$, mas a velocidade linear $\dot{x}$ é aumentada e, também, a velocidade angular média do motor para $\dot{\phi}=623.1 \mathrm{rad} / \mathrm{s}$ ou $\dot{\phi}=5950 \mathrm{rpm}$ que é a rotação nominal do motor $\left(\Omega_{0}\right)$.

A figura 54 mostra os resultados numéricos no regime permanente das curvas de amplitude e frequência. As curvas são plotadas para a voltagem do motor (frequência do motor) crescendo (asterisco, $*$ ) e decrescendo (círculo, o). Na figura a voltagem do motor está na faixa de $V=0.3 \mathrm{~V}\left(V_{m}{ }^{*}=0.1121 \mathrm{Nm}\right)$ até $V=2.0 \mathrm{~V}\left(V_{m}{ }^{*}=0.7476 \mathrm{Nm}\right)$ com variação de $\Delta V=0.01 \mathrm{~V}$. A figura $54 \mathrm{~b}$ representa uma visão amplificada da figura $54 \mathrm{a}$ onde pode-se observar que a ressonância ocorre em $\dot{\phi} \approx \omega^{*}=8.55 \mathrm{rad} / \mathrm{s}$. Observa-se na figura 54 que os mesmos valores são obtidos independentemente da variação da direção da voltagem (frequência) e nesse caso o efeito Sommerfeld não é observado nitidamente, e isso se deve ao fato do oscilador linear (sem impacto) estar sendo excitado por um motor com alta potência (ver Figs. 54a e 54b).

A seguir o mesmo sistema mecânico é analisado impactando uma parede rígida localizada a uma determinada distância. Quando o bloco está em contato com a parede o sistema mecânico é alterado da forma que o mesmo acopla com a parede através de um oscilador com uma alta rigidez e um alto amortecimento. Os parâmetros de colisão utilizados nas simulações numéricas estão listados na Tabela 19. O bloco é feito de aço e colide com uma parede de contreto localizada a uma distância de $G=1.5 \mathrm{~mm}$. Utilizando os dados da Tabela 19 são calculados os seguintes parâmetros: módulo de Young efetivo, $E_{\text {eff }}=31.456 \mathrm{GPa}$, massa efetiva, $m_{\text {eff }}=3.88 \mathrm{~kg}$, raio de curvatura efetivo, $r_{\text {eff }}=0.0725 \mathrm{~m}$, rigidez não linear da parede, $k_{I}=1.129 \times 10^{10} \mathrm{~N} / \mathrm{m}^{3 / 2}$ (Eq. 4.38) e coeficiente de amortecimento não-linear da parede, $c_{I}=3009.96 \mathrm{~kg} /\left(\mathrm{sm}^{1 / 4}\right)$ (Eq. 4.49). 


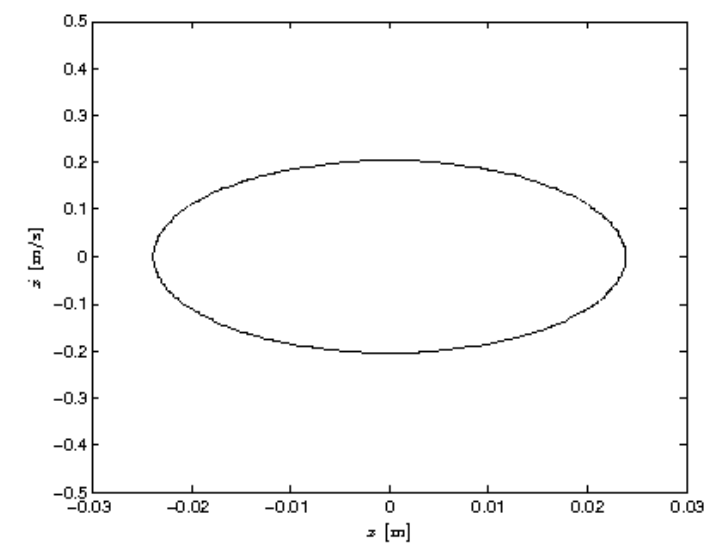

(a)

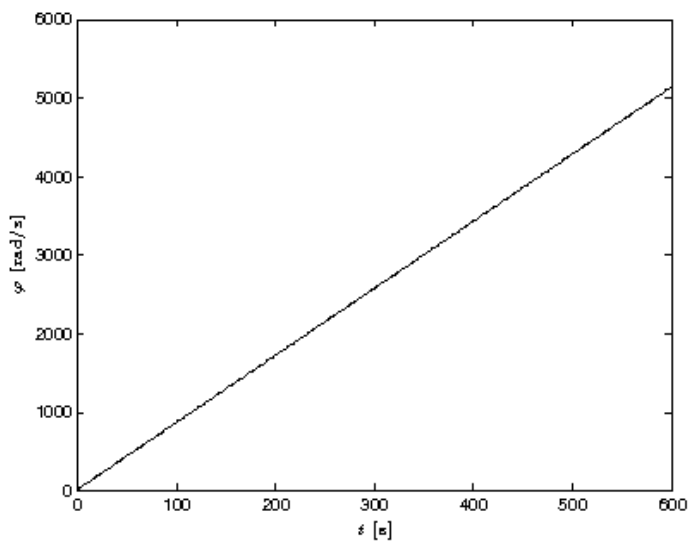

(b)

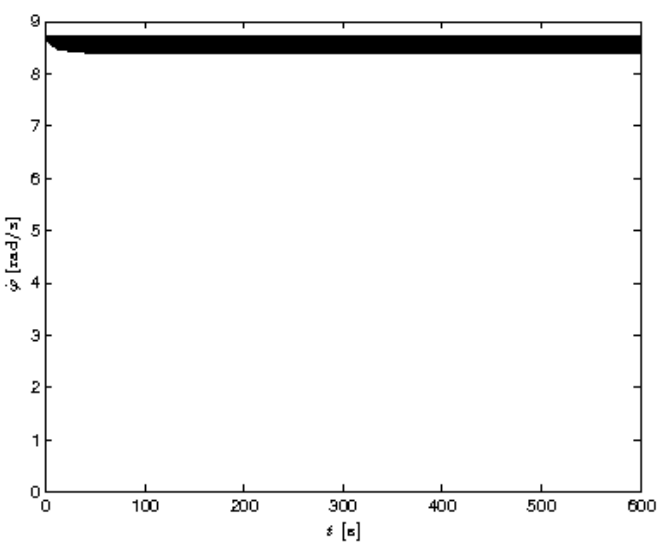

(c)

Figura 52 - Diagramas para $V=0.336 \mathrm{~V}$ : (a) retrato de fase; (b) histórico no tempo $\phi-t$; (c) histórico no tempo $\dot{\phi}-t$.

Tabela 19 - Parâmetros de Colisão.

\begin{tabular}{ll}
\hline Distância do impacto & $G=1.5 \mathrm{~mm}$ \\
Massa específica do bloco & $\rho=7850 \mathrm{~kg} / \mathrm{m}^{3}$ \\
Módulo de Young do bloco & $E=210 \mathrm{GPa}$ \\
Coeficiente de Poisson do bloco & $\sigma=0.29$ \\
Raio de curvatura do bloco & $r=0.0725 \mathrm{~m}$ \\
Massa específica da parede & $\rho_{w}=2500 \mathrm{~kg} / \mathrm{m}^{3}$ \\
Módulo de Young da parede & $E_{w}=35 \mathrm{GPa}$ \\
Coeficiente de Poisson da parede & $\sigma_{w}=0.2$ \\
Coeficiente de restituição na direção normal entre o bloco e a parede & $e_{n}=0.98$ \\
\hline
\end{tabular}




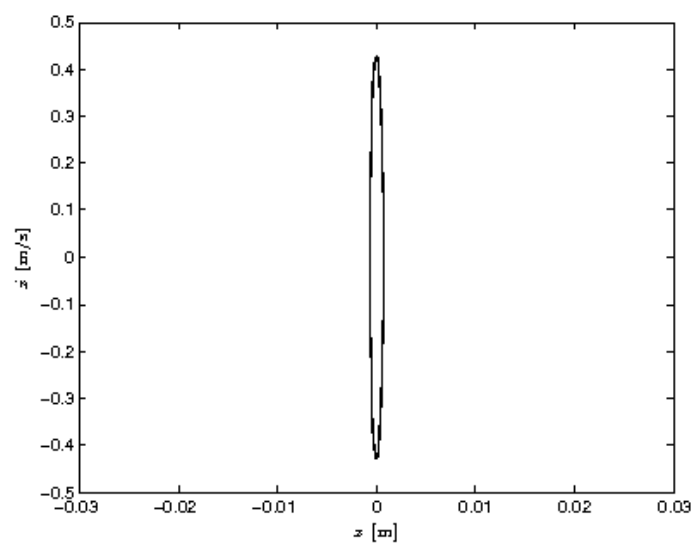

(a)

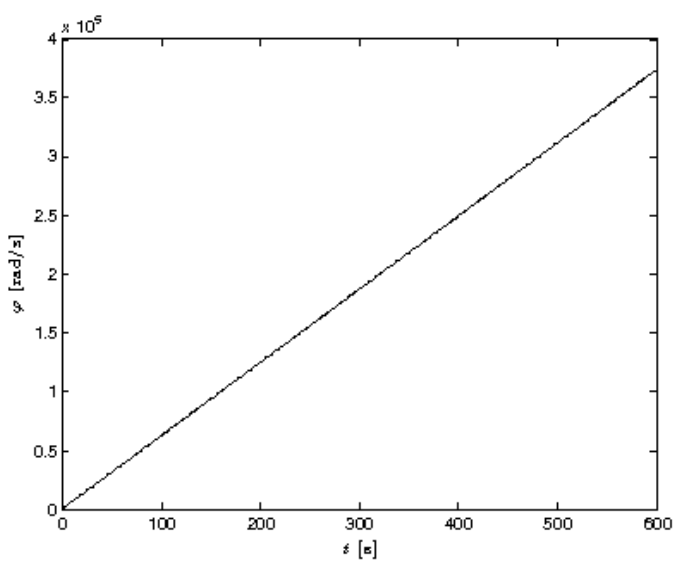

(b)

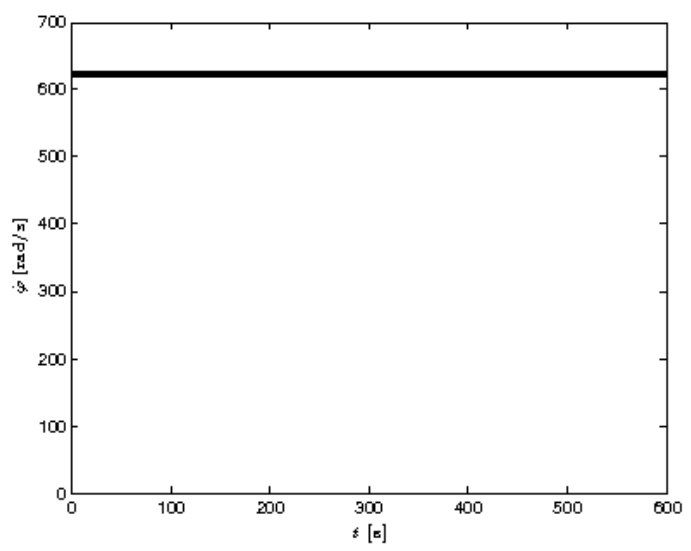

(c)

Figura 53 - Diagramas para $V=24.0 \mathrm{~V}$ : (a) retrato de fase; (b) histórico no tempo $\phi-t$; (c) histórico no tempo $\dot{\phi}-t$.

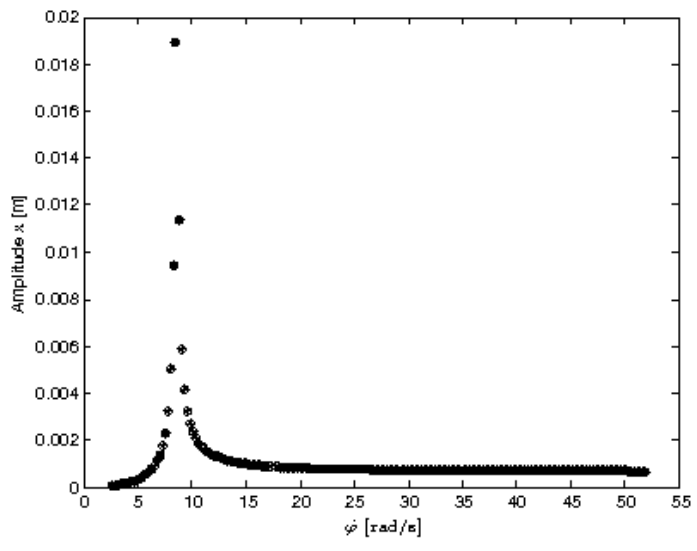

(a)

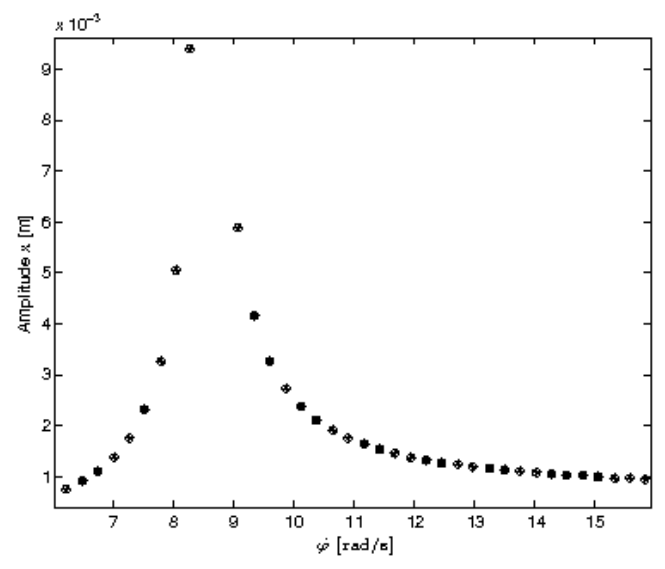

(b)

Figura 54 - Curvas de amplitude-frequência: (a) $5 . .50 \mathrm{rad} / \mathrm{s}$; (b) $7 . .15 \mathrm{rad} / \mathrm{s}$ (amplificação). 
A figura 55 ilustra o diagrama de histórico no tempo $x-t$, sendo que na Fig. $55 \mathrm{a}$ o tempo total de simulação escolhido é de $600 \mathrm{~s}$ e na Figura $55 \mathrm{~b}$ é de $10 \mathrm{~s}$ ( $V=0.336 \mathrm{~V}$, $\left.\dot{\phi} \approx \omega^{*}\right)$. O valor máximo do deslocamento $x$ é igual a $1.5 \mathrm{~mm}$, onde o contato ocorre durante um pequeno intervalo de tempo. Durante esse pequeno $\Delta t$, o sistema mecânico fica conectado a outro sistema não-linear do tipo massa mola amortecedor. Observa-se na Figura 55b que o contato com a parede ocorreu por nove vezes. No regime permanente, o valor máximo do deslocamento ocorre à esquerda do movimento $\approx-0.0025 \mathrm{~m}$ (p.ex., em $t=511 \mathrm{~s})$.

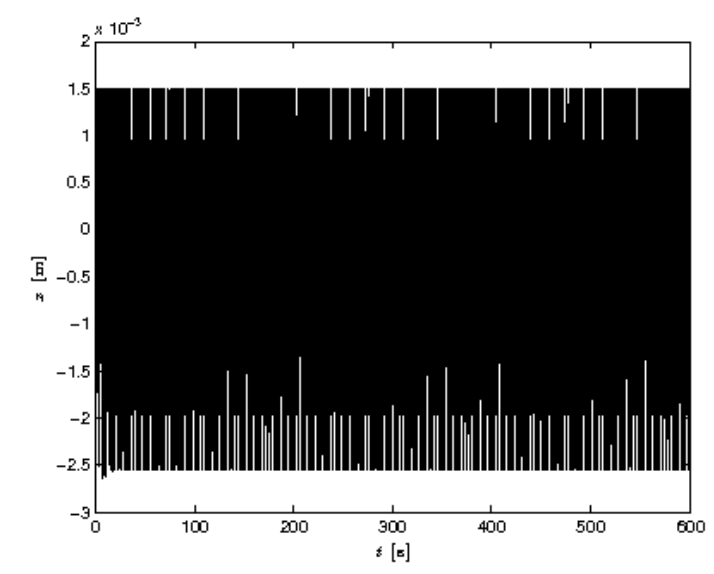

(a)

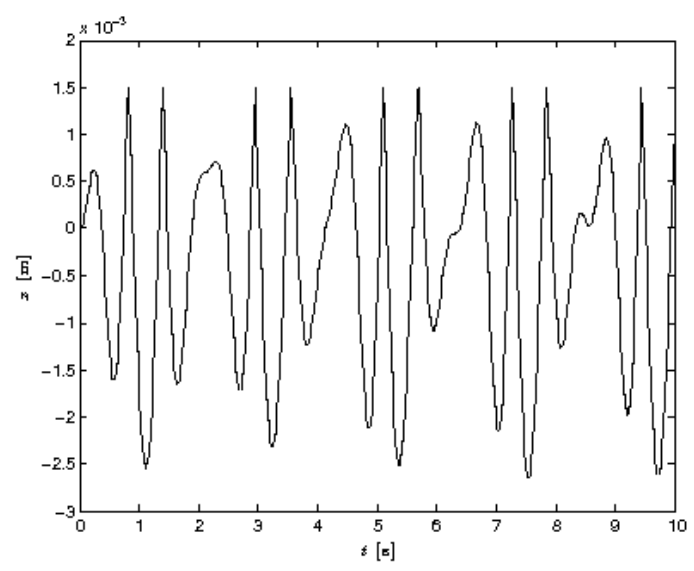

(b)

Figura 55 - Curvas de histórico no tempo $(V=0.336 \mathrm{~V})$ : (a) $0 \leq t \leq 600 \mathrm{~s}$; (b) $0 \leq t \leq$ $10 \mathrm{~s}$.

A figura 56 mostra os diagramas de planos de fase para uma voltagem do motor de $V=0.336 \mathrm{~V}$ equivalente a um torque de $V_{m}{ }^{*}=0.1256 \mathrm{Nm}$. O valor da posição da parede $x=1.5 \mathrm{~mm}$ é o limite positivo do deslocamento. Quando o sistema sofre impacto a não-linearidade da parede resulta também em variação do deslocamento à esquerda do bloco limitando-o (ver Figs. 55a e 56a). A figura 56b ilustra uma amplificação (região retangular marcada na Figura 56a) onde podemos observar no plano de fase no regime diversas linhas em órbitas.

A figura 57 apresenta a curva de amplitude-frequência obtida numericamente do sistema mecânico impactando na parede considerando a amplitude do movimento à esquerda. O parâmetro de controle voltagem do motor elétrico $V$ é mantido até que se alcance o regime permanente e após é alterado com uma variação de $\Delta V=0.01 \mathrm{~V}$ para se obter o novo regime permanente. A região de variação de $\dot{\phi}$ plotada na Figura 57 representa onde ocorre ressonância. Os valores numéricos obtidos para uma determinada de voltagem $V$ são utilizados como condições iniciais para um próximo valor de voltagem. Observa-se que para um aumento lento da rotação do motor, a amplitude de vibração do sistema aumenta até o ponto "A" $(V=0.66 \mathrm{~V}, x=0.04 \mathrm{~m}, \dot{\phi}=14.89 \mathrm{rad} / \mathrm{s})$. Para o próximo valor da voltagem a amplitude de $x$ é muito menor do que no ponto "A". Assim, incrementando 


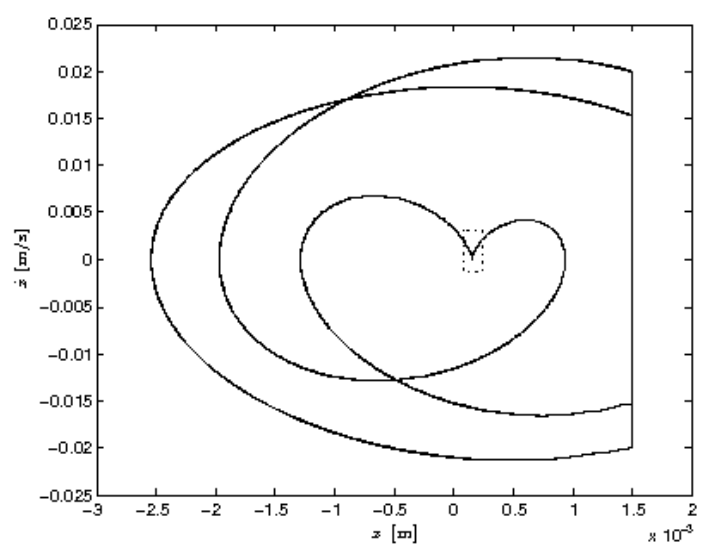

(a)

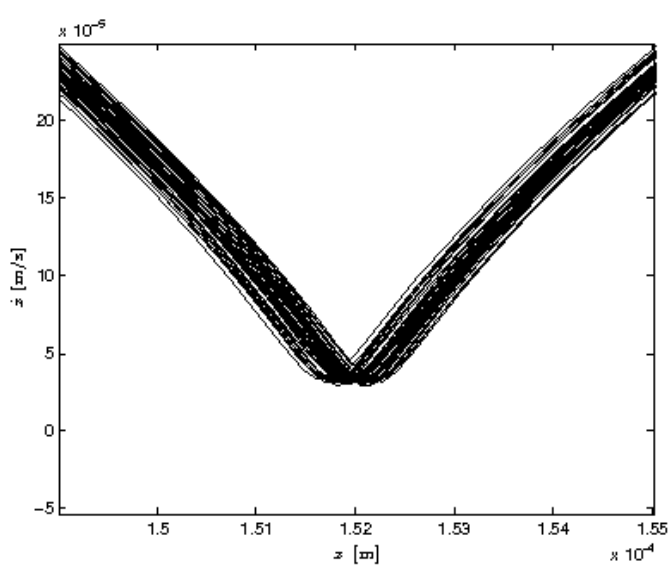

(b)

Figura 56 - Diagramas de planos de fase: (a) $V=0.336 \mathrm{~V}$; (b) $V=0.336 \mathrm{~V}$ (amplificação).

o parâmetro de controle $V$ causa uma diminuição da amplitude de $x$ e um aumento da frequência - ponto "B" ( $V=0.67 \mathrm{~V}, x=0.000899 \mathrm{~m}, \dot{\phi}=17.4 \mathrm{rad} / \mathrm{s})$. Após o ponto "B" o aumento do parâmetro de controle causa um decréscimo na amplitude com aumento da rotação do motor. No sentido inverso, isto é, decrementando lentamente a rotação do motor, a amplitude de vibração movimenta à esquerda ao longo da curva de amplitude-frequência (Fig. 57) até o ponto "C" $(V=0.46 \mathrm{~V}, x=0.0014 \mathrm{~m}, \dot{\phi}=11.95 \mathrm{rad} / \mathrm{s})$. Para o próximo valor de voltagem uma mudança no padrão de comportamento da amplitude $x$ aparece o ponto "D" ( $V=0.45 \mathrm{~V}, x=0.003 \mathrm{~m}, \dot{\phi}=11.66 \mathrm{rad} / \mathrm{s})$, sendo a amplitude aumentada lentamente e a frequência alterada em salto. Após o ponto "D", o parâmetro de controle produz um decrescimento na amplitude e na rotação do motor. Os saltos entre os pontos "A" e "B" e entre os pontos "C" e "D" caracterizam o efeito Sommerfeld que foi observado no sistema mecânico não-ideal com não-linearidade introduzida no contato do bloco com a parede. 


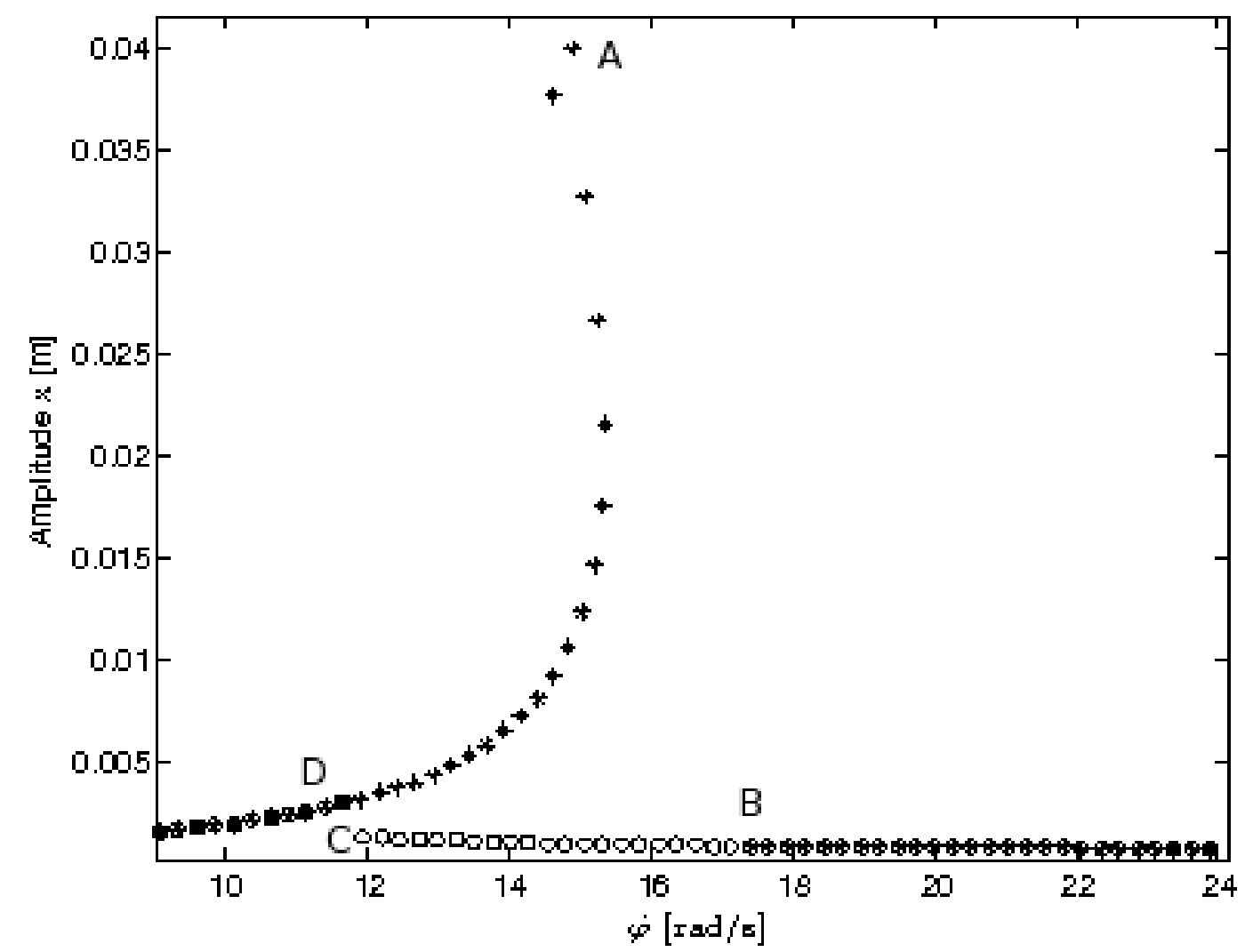

Figura 57 - Curva de amplitude-frequência (asteriscos - para aumento de $V$; Círculos para diminuição de $V$ ). 


\section{Conclusões e Recomendações}

A presente tese trata do estudo de não-linearidades racionais existentes na modelagem de osciladores mecânicos com a proposta de soluções aproximadas e análises numéricas para problemas específicos. Dessa forma, dentro da vasta gama de aplicações, descreve-se no presente trabalho problemas de vibração livre em mecânica não-linear (ver Capítulo 3), problemas envolvendo modelos de contato usando a abordagem de esfera-contato (ver Capítulo 4), e análise do efeito Sommerfeld em problemas não-ideais lineares e não-lineares envolvendo contato (ver Capítulo 5). Os desenvolvimentos apresentados nesses capítulos foram obtidos durante a realização de várias pesquisas (como por exemplo, Navarro e Braun (2013), Navarro, Balthazar e Brasil (2014), Navarro e Cveticanin (2016), Jordam-Caserta, Navarro e Cabezas-Gómez (2016), entre outros estudos).

Retomando o objetivo principal desta tese, isto é, o de estudar a colisão de osciladores mecânicos não-ideais com não-linearidade de ordem fracionária, verifica-se que o mesmo foi alcançado com os estudos apresentados nos Capítulos 3, 4 e 5, cujas conclusões são detalhadas a seguir.

O capítulo 3 trata do estudo de vibração livre de osciladores mecânicos altamente não-lineares com ordem de não-linearidade inteira ou fracionária. Na seção 3.1 apresenta-se um estudo de vibração livre em osciladores conservativos com condições iniciais particulares. Nessa seção utilizou-se uma metodologia denominada de abordagem Hamiltoniana, proposta por He (2010), para a obtenção de soluções aproximadas dos osciladores mecânicos. Essa abordagem foi aplicada em equações com soma de não-linearidades não-inteiras. A solução aproximada proposta é na forma trigonométrica, sendo que com a utilização da referida abordagem obtém-se uma relação entre a amplitude e a frequência de oscilação. A solução aproximada foi comparada em problemas com um ou mais termos conservativos. Foi proposto um procedimento para a estimativa dos erros médios e o mesmo foi aplicado em diversos casos exemplos. Com base nos resultados obtidos pode-se concluir o seguinte:

1. Para osciladores com um termo conservativo não-linear e ordem de não-linearidade $\alpha$ até 5.0, o período aproximado de vibração fornece valores precisos quando comparados com os períodos obtidos numericamente. Para valores maiores de $\alpha$ o período de vibração diverge. Para estender os valores de $\alpha$ recomenda-se utilizar outras ordens para a função trigonométrica.

2. Os erros calculados para o período de vibração dependem dos parâmetros do sistema, da ordem de não-linearidade e da amplitude inicial. Observa-se que, para valores extremamente altos da amplitude de vibração, a variação da amplitude tem influência desprezível no erro entre os períodos de vibração aproximado e exato para um determinado 
oscilador mecânico. Para valores altos de amplitude, o valor limite do erro depende das propriedades do oscilador.

3. Utilizando uma função trigonométrica de primeira ordem, o erro relativo médio mostrou que a forma da solução aproximada altera muito rapidamente quando a ordem de não-linearidade é muito alta ou pequena em relação à linear, produzindo altos valores de erros. O oscilador conservativo não-linear puro apresenta um valor limite (máximo) do erro relativo (ver Tabela 2). Por exemplo, para um oscilador quadrático puro, o erro limite é 0.12047 e para o oscilador cúbico o erro é de 0.23385 .

Na seção 3.2 apresenta-se uma generalização da seção 3.1, isto é, a análise de problemas de vibração livre de osciladores conservativos modelados com equações diferenciais com condições iniciais gerais. É proposto um método para obter a relação da frequência de oscilação em função da amplitude inicial, da velocidade inicial e dos parâmetros do sistema. Investiga-se, também, um procedimento para a estimativa do erro médio entre a solução aproximada e a exata. São propostos dois algoritmos computacionais como alternativas para a determinação da frequência, amplitude, fase e erros relativos das soluções quando as soluções analíticas são complexas ou não estão disponíveis. Considerando os resultados obtidos na presente seção conclui-se o seguinte:

1. Os osciladores mecânicos governados pela equação de Duffing possuem um erro relativo para o período de vibração, $\Delta_{T}$, que converge para $2.172 \%$ quando $(A \rightarrow \infty, v)$ e para $8.264 \%$ quando $(A=0, v \rightarrow \infty)$. Nesse mesmo tipo de oscilador, o erro relativo médio, $\Delta$, converge para 0.233854 quando $(A \rightarrow \infty, v)$ e para 0.282597 quando $(A=0, v \rightarrow \infty)$.

2. No caso de osciladores conservativos não-lineares puros, os erros relativos para o período de vibração, $\Delta_{T}$, e para a solução média, $\Delta$, diminuem com o aumento de $\alpha$ até $\alpha=1$, onde os erros são nulos, e além desse ponto $(\alpha>1)$ os erros relativos começam a aumentar. De acordo com a Tabela 8 , onde a velocidade inicial é nula $(v=0)$ existe um limite ou máximos valores para os erros, isto é, $\Delta_{T}(A, 0)=\Delta_{T}(A \rightarrow \infty, v)$ e $\Delta(A, 0)=\Delta(A \rightarrow \infty, v)$. Os valores mínimos para os erros relativos ocorrem para valores menores de $A$ com o aumento da potência $\alpha$.

A seção 3.3 mostra um procedimento, baseado na expansão em série de Taylor, para determinar soluções aproximadas de osciladores mecânicos não-lineares com nãolinearidades inteira e não-inteira. A ideia de utilizar séries infinitas para resolver equações diferenciais é de Newton (ver (NEWTON, 1671) e (HAIRER; NØRSETT; WANNER, 1993)). A solução é dada pela expansão truncada de Taylor em torno de um determinado instante, ver (BERVILLIER, 2012). Na presente tese aplicou-se esse procedimento para equações com não-linearidade não-inteira (ver Eq. 3.141) utilizando os coeficientes de uma série de potência derivada por Euler ((EULER, 1748, parágrafo 76), ver também (KNUTH, 1997), (HENRICI, 1956) e (HAIRER; NØRSETT; WANNER, 1993)). O procedimento proposto foi aplicado em três osciladores mecânicos (um conservativo com não-linearidade 
fracionária, um não-conservativo com não-linearidade cúbica e outro não-conservativo com não-linearidade fracionária). Comparando as soluções aproximadas com as numéricas obtidas por Runge-Kutta, observa-se uma grande acuracidade na solução para longos intervalos de tempo. Entretanto, como a solução tem a base na expansão em série, além da dificuldade de convergência longe do instante de aproximação, mesmo aplicando os aproximantes de Padé pode ser que a técnica não seja eficaz para outros tipos de osciladores, isto é, com parâmetros de simulação diferentes ou variações nos modelos dos osciladores mecânicos.

No capítulo 4 é apresentado um método para o cálculo de forças de contato entre corpos através de um modelo desenvolvido por Cundall e Strack (1979), denominado de modelo de "esfera-suave". Esse modelo é composto por um sistema massa-mola-amortecedor e são estudados os parâmetros cinemáticos da colisão (posição, velocidade e aceleração) para sistemas lineares e não-lineares. Para o uso do modelo linear deve-se inferir os coeficientes de rigidez e de amortecimento. O cálculo do coeficiente de amortecimento é determinado em função do coeficiente de restituição e da rigidez. Para a estimativa do coeficiente de rigidez (modelo linear) é proposta uma analogia com os modelos não-lineares de três modos diferentes, isto é, através da sobreposição máxima do impacto (Eq. 4.96), da duração do contato (Eq. 4.101) e da sobreposição média do impacto (Eq. 4.104). No caso não-linear são analisados dois sub-modelos que diferem pela forma de cálculo da força dissipativa, denominados no presente trabalho de modelos A e B. Para esses modelos o coeficiente de rigidez é calculado através das propriedades dos materiais usando a teoria de Hertz. O modelo A dispõe de solução analítica para se calcular o coeficiente de amortecimento não-linear. O modelo não-linear proposto por Hunt e Grossley (1975) (denominado de modelo B) que é função da velocidade de impacto e não apresenta descontinuidades entre o início e o fim do contato, apresenta somente soluções aproximadas para o cálculo do coeficiente de amortecimento. No caso desse modelo não-linear B são derivadas duas novas relações, uma para o cálculo de coeficiente não-linear de amortecimento (Eq.4.86, ver (JORDAM-CASERTA; NAVARRO; CABEZAS-GóMEZ, 2016)) e outra para o cálculo do tempo de contato (Eq.4.87) em que os erros percentuais em relação às soluções numéricas são muito pequenos para a faixa de aplicação proposta. Os modelos analisados, linear e os dois não-lineares (A e B), são aplicados a um problema dinâmico simples de uma esfera em queda livre impactando sobre uma superfície rígida.

O capítulo 5 apresenta o estudo da vibração de um sistema mecânico não-ideal colidindo em uma parede rígida. A característica de elasticidade do sistema mecânico não tem limitações, isto é, pode ser linear, cúbica, com não-linearidade pequena ou grande, ou com qualquer ordem de não-linearidade (inteira ou fracionária). Esse tipo de problema também foi estudado por Cveticanin e Zukovic (2015a) sendo que nesta tese foi incorporada a colisão, isto é, foi analisado o mesmo tipo de sistema não-ideal podendo sofrer impacto em uma parede rígida. A modelagem do impacto foi feita através da incorporação ao 
sistema mecânico original de um outro oscilador não-linear contendo elementos de mola e amortecedor, similarmente ao Método dos Elementos Discretos para sistemas particulados modelados com o mecanismo "esfera-suave" (ver capítulo 4). O efeito Sommerfeld foi obtido analiticamente e numericamente em um sistema mecânico não-ideal com rigidez linear e não-linear. A metodologia desenvolvida foi aplicada também em um sistema contendo valores dimensionais para um motor elétrico da Maxon. O método estudado nesse capítulo pode ser estendido para outros problemas de vibro-impacto e para estudos experimentais.

Considerando os estudos realizados na presente tese seguem-se as seguintes recomendações de trabalhos futuros:

1. Desenvolver um procedimento aproximado para analisar problemas de vibração forçada de osciladores conservativos não-lineares com não-linearidades racionais modelados com equações diferenciais com condições iniciais gerais.

2. Aplicar o procedimento baseado na expansão em série de Taylor para outros osciladores mecânicos visando a generalização desse procedimento, pois este tem dificuldade de convergência mesmo considerando os aproximantes de Padé. Aplicar também essa técnica para osciladores não-conservativos com não-linearidades racionais sujeitos à excitação forçada.

3. Fazer a caracterização analítica da curva de amplitude-frequência na presença do impacto e, também, estudar o efeito Sommerfeld experimentalmente comparando com os resultados obtidos no capítulo 5 .

4. Aplicar os diversos modelos de contato desenvolvidos no capítulo 4 para o estudo de problemas de vibração de osciladores mecânicos não-ideais com colisão. Um modelo a ser testado é o modelo contínuo especificado pela Eq. (4.70). Além da análise de problemas envolvendo colisão normal, estudada no capítulo 4, desenvolver outros trabalhos em que os sistemas mecânicos estejam sujeitos às colisões oblíquas. 


\section{Referências}

ADOMIAN, G. Nonlinear Stochastic Operator Equations. Orlando, Florida, USA: Academic Press Inc., 1986.

AGUILERA-NAVARRO, M. C. K. et al. Os aproximantes de padé. Matemática Universitária, n. 26/27, p. 49-66, 1999.

AKBARZADE, M.; KARGAR, A. Accurate analytical solutions to nonlinear oscillators by means of the Hamiltonian approach. Mathematical Methods in the Applied Sciences, John Wiley \& Sons, Ltd, v. 34, n. 17, p. 2089-2094, 2011.

AKBARZADE, M.; KARGAR, A. Application of the Hamiltonian approach to nonlinear vibrating equations. Mathematical and Computer Modelling, v. 54, n. 9-10, p. $2504-2514$, 2011.

AKBARZADE, M.; KHAN, Y. Dynamic model of large amplitude non-linear oscillations arising in the structural engineering: analytical solutions. Mathematical and Computer Modelling, v. 55, n. 3-4, p. $480-489,2012$.

AKBARZADE, M.; LANGARI, J. Solution of nonlinear oscillators using global error minimization method. Adv. Studies Theor. Phys, v. 5, p. 349-356, 2011.

ALDER, B. J.; WAINWRIGHT, T. E. Phase transition for a hard sphere system. The Journal of Chemical Physics, AIP, v. 27, n. 5, p. 1208-1209, 1957.

ALVES, J. et al. A comparative study of the viscoelastic constitutive models for frictionless contact interfaces in solids. Mechanism and Machine Theory, v. 85, p. 172 $188,2015$.

AMORE, P.; ARANDA, A. Improved lindstedt-poincaré method for the solution of nonlinear problems. Journal of Sound and Vibration, Elsevier, v. 283, p. 1115-1136, 2005.

ANTYPOV, D.; ELLIOTT, J. A. On an analytical solution for the damped Hertzian spring. Europhysics Letter, v. 94, n. 5, p. 50004, 2011.

ARIO, I.; WATSON, A. Structural stability of multi-folding structures with contact problem. Journal of Sound and Vibration, v. 324, n. 1-2, p. 263 - 282, 2009.

ASKEY, R. A.; ROY, R. Gamma function. In: OLVER, F. W. J. et al. (Ed.). NIST Handbook of Mathematical Functions. New York, NY, USA: National Institute of Standards and Technology, Cambridge University Press, 2010. cap. 5, p. 135-147.

ATANACKOVIC, T.; ACHENBACH, M. A least square solution and error estimate for some vibration problems. ZAMM, Wiley Online Library, v. 65, n. 1, p. 57-59, 1985.

BAKER, G. A. Essentials of Padé Approximants. New York, NY, USA: Academic Press, 1975. 
BALTHAZAR, J. et al. A review of new vibration issues due to non-ideal energy sources. In: UDWADIA, F. E.; WEBER, H. I.; LEITMAN, G. (Ed.). Dynamics Systems and Control (Stability and Control: Theory. Methods and Applications). Boca Raton, FL: CRC Press, 2004. v. 22, p. 237-258.

BALTHAZAR, J.; PONTES, B. On friction induced nonideal vibrations: A source of fatigue. In: INMAN, D. J. et al. (Ed.). Damage Prognosis-For Aerospace, Civil and Mechanical Systems. New York, NY: John Wiley and Sons, 2005.

BAYAT, M.; PAKAR, I. On the approximate analytical solution to non-linear oscillation systems. Shock and vibration, IOS Press, v. 20, n. 1, p. 43-52, 2013.

BAYAT, M.; PAKAR, I.; CVETICANIN, L. Nonlinear free vibration of systems with inertia and static type cubic nonlinearities: an analytical approach. Mechanism and Machine Theory, Elsevier, v. 77, p. 50-58, 2014.

BELATO, D. et al. Chaotic vibrations of a nonideal electro-mechanical system. International Journal of Solids and Structures, v. 38, p. 1699-1706, 2001.

BELENDEZ, A. et al. Notes on "Application of the Hamiltonian approach to nonlinear oscillators with rational and irrational elastic terms". Mathematical and Computer Modelling, v. 54, n. 11-12, p. $3204-3209,2011$.

BENYAHIA, S.; GALVIN, J. E. Estimation of numerical errors related to some basic assumptions in discrete particle methods. Industrial \& Engineering Chemistry Research, v. 49, n. 21, p. 10588-10605, 2010.

BERVILLIER, C. Status of the differential transformation method. Applied Mathematics and Computation, v. 218, n. 20, p. 10158 - 10170, 2012.

BLAZEJCZYK-OKOLEWSKA, B. et al. Chaotic Mechanics in Systems with Impacts and Friction. Singapore: World Scientific, 1999. v. 36. (Series on Nonlinear Science Series A, v. 36$)$.

BUCHHOLTZ, V.; PöSCHEL, T. Numerical investigations of the evolution of sandpiles. Physica A, v. 202, p. 390-401, 1994.

BYRD, P. F.; FRIEDMAN, M. D. Handbook of Elliptic Integrals for Engineers and Scientists. [S.l.]: Springer-Verlag Berlin Heidelberg, 1971. (Die Grundlehren der mathematischen Wissenschaften, 67).

CAMPBELL, C. Shear flows in granular material. Tese (PhD Thesis) - California Institute of Technology, 1982.

CHATTERJEE, S.; MALLIK, A.; GHOSH, A. On impact dampers for non-linear vibration systems. Journal of Sound and Vibration, v. 187, n. 3, p. 403 - 420, 1995.

CHEN, F.; DRUMM, E. C.; GUIOCHON, G. Prediction/verification of particle motion in one dimension with the discrete-element method. International Journal of Geomechanics, v. 7, p. 344-352, 2007.

COPPOLA, V.; RAND, R. Averaging using elliptic functions: approximation of limit cycles. Acta Mechanica, Springer-Verlag, v. 81, n. 3-4, p. 125-142, 1990. 
CROWE, C.; SOMMERFELD, M.; TSUJI, Y. Multiphase Flows with Droplets and Particles. Boca Raton: CRC Press, 1997.

CUNDALL, P. A.; STRACK, O. D. L. A discrete numerical model for granular assemblies. Géotechnique, v. 29, n. 1, p. 47-65, 1979.

CUNNINGHAM, W. J. Introduction to Nonlinear Analysis. New York, NY, USA: McGraw-Hill Book Company, Inc., 1958.

CVETICANIN, L. The approximate solving methods for the cubic duffing equation based on the jacobi elliptic functions. International Journal of Nonlinear Sciences and Numerical Simulation, v. 10, n. 11-12, p. 1491-1516, 2009.

CVETICANIN, L. Oscillator with fraction order restoring force. Journal of Sound and Vibration, v. 320, n. 4-5, p. $1064-1077,2009$.

CVETICANIN, L. Dynamics of the non-ideal mechanical systems: a review. Journal of the Serbian Society for Computational Mechanics, p. 75-86, 2010.

CVETICANIN, L. On the truly nonlinear oscillator with positive and negative damping. Applied Mathematics and Computation, v. 243, p. 433 - 445, 2014.

CVETICANIN, L.; KALAMI-YAZDI, M.; ASKARI, H. Analytical approximations to the solutions for a generalized oscillator with strong nonlinear terms. Journal of Engineering Mathematics, v. 77, n. 1, p. 211-223, 2012.

CVETICANIN, L. et al. Application of Hamiltonian approach to the generalized nonlinear oscillator with fractional power. International Journal of Nonlinear Sciences and Numerical Simulation, v. 11, n. 12, p. 997-1002, 2010.

CVETICANIN, L.; KOVACIC, I.; RAKARIC, Z. Asymptotic methods for vibrations of the pure non-integer order oscillator. Computers $\&$ Mathematics with Applications, v. 60, n. 9 , p. $2616-2628,2010$.

CVETICANIN, L.; POGANY, T. Oscillator with a sum of noninteger-order nonlinearities. Journal of Applied Mathematics, Hindawi Publishing Corporation, v. 2012, n. 649050, p. $1-20,2012$.

CVETICANIN, L.; ZUKOVIC, M. Motion of a motor-structure non-ideal system. European Journal of Mechanics - A/Solids, v. 53, p. 229 - 240, 2015.

CVETICANIN, L.; ZUKOVIC, M. Non-ideal mechanical system with an oscillator with rational nonlinearity. Journal of Vibration and Control, v. 21, p. 2149-2164, 2015.

DAVIES, H. G. Random vibration of a beam impacting stops. Journal of Sounde and Vibration, v. 68, n. 4, p. 479-487, 1980.

DIMENTBERG, M. et al. Dynamics of an unbalanced shaft interacting with a limited power supply. Nonlinear Dynamics, Kluwer Academic Publishers, v. 13, n. 2, p. 171-187, 1997.

DURMAZ, S.; DEMIRBAG, S. A.; KAYA, M. High order Hamiltonian approach to nonlinear oscillators. International Journal of Nonlinear Sciences and Numerical Simulation, v. 11, n. 8, p. 565-570, 2010. 
DURMAZ, S.; DEMIRBAG, S. A.; KAYA, M. Hamiltonian approach to multiple coupled nonlinear oscillators. Acta Physica Polonica A, v. 121, n. 1, p. 47-49, 2012.

DURY, C. M.; RISTOW, G. H. Radial segregation in a two-dimensional rotating drum. J. Phys. I France, v. 7, n. 5, p. 737-745, 1997.

EL-SHAHED, M. Application of differential transform method to non-linear oscillatory systems. Communications in Nonlinear Science and Numerical Simulation, v. 13, n. 8, p. $1714-1720,2008$.

EULER, L. Introductio in Analysin Infinitorum. Lausannae: Academia Imperialis Scientiarum, 1748. VIII. (1 - Opera Mathematica, VIII).

FALCON, E. et al. Behavior of one inelastic ball bouncing repeatedly off the ground. The European Physical Journal B - Condensed Matter and Complex Systems, v. 3, n. 1, p. 45-57, 1998.

FARZANEH, Y.; TOOTOONCHI, A. A. Global error minimization method for solving strongly nonlinear oscillator differential equations. Computers $\& 5$ Mathematics with Applications, Elsevier, v. 59, p. 2887-2895, 2010.

FIDLIN, A. Nonlinear Oscillations in Mechanical Engineering. Berlin, Heidelberg: Springr-Verlag, 2006.

FINLAYSON, B. A. The Method of Weighted Residuals and Variational Principles. New York, NY, USA: Academic Press, 1972.

FLORES, P. et al. On the continuous contact force models for soft materials in multibody dynamics. Multibody System Dynamics, v. 25, p. 357-375, 2011.

FOERSTER, S. F. et al. Measurements of the collision properties of small spheres. Physics of Fluids, v. 6, n. 3, p. 1108-1115, 1994.

GARG, R. et al. Documentation of open-source MFIX-DEM software for gas-solids flows. Morgantown, WV, USA, 2010. Disponível em: < https://mfix.netl.doe.gov/documentation/ dem_doc_2010.pdf $>$.

GARG, R. et al. Open-source MFIX-DEM software for gas-solids flows: Part I-Verification studies. Powder Technology, v. 220, p. 122 - 137, 2012.

GOLDSCHMIDT, M. J. V.; BEETSTRA, R.; KUIPERS, J. A. M. Hydrodynamic modelling of dense gas-fluidised beds: Comparison and validation of $3 \mathrm{~d}$ discrete particle and continuum models. Powder Technology, v. 142, n. 1, p. 23 - 47, 2004.

GOLDSMITH, W. Impact. London, England: Edward Arnold Publishers Ltd., 1960.

GOPALAKRISHNAN, P.; TAFTI, D. Development of parallel DEM for the open source code MFIX. Powder Technology, v. 235, p. 33 - 41, 2013.

HAFF, P. K.; WERNER, B. T. Computer simulation of the mechanical sorting of grains. Powder Technology, v. 48, n. 3, p. 239 - 245, 1986.

HAIRER, E.; NØRSETT, S. P.; WANNER, G. Solving ordinary differential equations I: nonstiff problems. Berlin, Heidelberg: Springer-Verlag, 1993. v. 8. (Springer Series in Computational Mathematics, v. 8). 
HE, J.-H. Homotopy perturbation technique. Computer Methods in Applied Mechanics and Engineering, Elsevier, v. 178, n. 3, p. 257-262, 1999.

HE, J.-H. Variational iteration method-a kind of non-linear analytical technique: some examples. International Journal of Non-linear Mechanics, Elsevier, v. 34, n. 4, p. 699-708, 1999.

HE, J.-H. Preliminary report on the energy balance for nonlinear oscillations. Mechanics Research Communications, Elsevier, v. 29, n. 2, p. 107-111, 2002.

HE, J.-H. Homotopy perturbation method: a new nonlinear analytical technique. Applied Mathematics and Computation, Elsevier, v. 135, n. 1, p. 73-79, 2003.

HE, J.-H. Variational approach for nonlinear oscillators. Chaos, Solitons 86 Fractals, Elsevier, v. 34, n. 5, p. 1430-1439, 2007.

HE, J.-H. Hamiltonian approach to nonlinear oscillators. Physics Letters, Section A: General, Atomic and Solid State Physics, v. 374, n. 23, p. 2312 - 2314, 2010.

HE, J.-H.; ZHONG, T.; L., T. Hamiltonian approach to duffing-harmonic equation. International Journal of Nonlinear Sciences and Numerical Simulation, v. 11, p. 43-46, 2010 .

HENRICI, P. Automatic computations with power series. J. ACM, ACM, New York, NY, USA, v. 3, n. 1, p. 10-15, 1956.

HERIŞANU, N.; MARINCA, V. Accurate analytical solutions to oscillators with discontinuities and fractional-power restoring force by means of the optimal homotopy asymptotic method. Computers $\&$ Mathematics with Applications, v. 60, n. 6, p. 1607 $1615,2010$.

HERTZ, H. Über die Berührung fester elastischer Körper. Journal für die Reine und Angewandte Mathematik, v. 92, p. 156 - 171, 1882.

HOEF, M. A. van der et al. Multiscale modeling of gas-fluidized beds. Advances in Chemical Engineering, v. 31, p. 65 - 149, 2006.

HOOMANS, B. P. B. et al. Discrete particle simulation of bubble and slug formation in a two-dimensional gas-fluidised bed: A hard-sphere approach. Chemical Engineering Science, v. 51, n. 1, p. 99 - 118, 1996.

HU, G. et al. On the determination of the damping coefficient of non-linear spring-dashpot system to model hertz contact for simulation by discrete element method. Journal of Computers, v. 6, n. 5, p. $984-988,2011$.

HUNT, K. H.; GROSSLEY, F. R. E. Coefficient of restitution interpreted as damping in vibroimpact. ASME Journal of Applied Mechanics, v. 7, p. 440 - 445, 1975.

IBRAHIM, R. A. Vibro-impact Dynamics: Modeling, Mapping and Applications. Berlin, Heidelberg: Springer-Verlag, 2009. v. 43. (Lecture Notes in Applied and Computational Mechanics, v. 43).

ING, J. et al. Bifurcation analysis of an impact oscillator with a one-sided elastic constraint near grazing. Physica D: Nonlinear Phenomena, v. 239, n. 6, p. 312 - 321, 2010. 
JANKOWSKI, R. Non-linear viscoelastic modelling of earthquake-induced structural pounding. Earthquake Engineering and Structural Dynamics, v. 34, n. 6, p. 595-611, 2005.

JIAO, Y. et al. An aftertreatment technique for improving the accuracy of adomian's decomposition method. Computers $\&$ Mathematics with Applications, v. 43, n. 6, p. $783-$ $798,2002$.

JOHNSON, K. L. Contact Mechanics. Cambridge, UK: Cambridge University Press, 1985.

JORDAM-CASERTA, A. Desenvolvimento de relações não-lineares para mecanismos de contato por meio de estudo analítico e numérico. Tese (Doutorado, 172p.) — Escola de Engenharia de São Carlos, Universidade de São Paulo, São Carlos, 2015.

JORDAM-CASERTA, A.; NAVARRO, H. A.; CABEZAS-GóMEZ, L. Damping coefficient and contact duration relations for continuous nonlinear spring-dashpot contact model in \{DEM\}. Powder Technology, v. 302, p. $462-479,2016$.

JUTTE, C. V. Generalized synthesis methodology of nonlinear springs for prescribed load-displacement functions. Tese (Doutorado) — The University of Michigan, 2008.

KAC, V.; CHEUNG, P. Quantum Calculus. New York, NY, USA: Springer-Verlag, 2002. (Universitext).

KETTERHAGEN, W. R. et al. Granular segregation in discharging cylindrical hoppers: A discrete element and experimental study. Chemical Engineering Science, v. 62, n. 22, p. $6423-6439,2007$.

KHAN, N. A.; JAMIL, M.; ARA, A. Multiple-parameter Hamiltonian approach for higher accurate approximations of a nonlinear oscillator with discontinuity. International Journal of Differential Equations, v. 2011, n. 649748, 2011.

KNUTH, D. E. The Art or Computer Programming: Seminumerical Algorithms. Third edition. Berkeley, California: Addison Wesley Longman, 1997. v. 2.

KOnOnEnKO, V. O. Vibrating System with a Limited Power Supply. London: Iliffe Books Ltd., 1969.

KOVACIC, I.; RAKARIC, Z.; CVETICANIN, L. A non-simultaneous variational approach for the oscillators with fractional-order power nonlinearities. Applied Mathematics and Computation, v. 217, n. 8, p. 3944 - 3954, 2010.

KRASNOPOLSKAYA, T. S.; SHVETS, A. Y. Chaotic interactions in a pendulum energy source system (in russian). Prikl. Mekh, v. 26, n. 5, p. 90-96, 1990.

KRASNOPOLSKAYA, T. S.; SHVETS, A. Y. Regular and chaotic surface waves in a liquid in a cylindrical tank (in russian). Prikl. Mekh, v. 26, n. 8, p. 85-93, 1990.

KRASNOPOLSKAYA, T. S.; SHVETS, A. Y. Chaos in dynamics of machines with a limited power supply. In: MECHANICAL ENGINEERING FACULT, CZECH TECHNICAL UNIVERSITY OF PRAGUE. Proceeding of the 8th World Congress on the Theory of Machines and Mechanisms. Prague, Czechoslovakia, 1991.

KRASNOPOLSKAYA, T. S.; SHVETS, A. Y. Chaos in vibrating systems with a limited power-supply. Chaos: An Interdisciplinary Journal of Nonlinear Science, v. 3, n. 3, p. 387-395, 1993. 
KRUGGEL-EMDEN, H. et al. Review and extension of normal force models for the discrete element method. Powder Technology, v. 171, n. 3, p. 157 - 173, 2007.

KRYLOV, N.; BOGOLIUBOV, N. N. Introduction to Nonlinear Mechanics. Princeton, N. J., USA: Princeton University Press, 1947.

KUWABARA, G.; KONO, K. Restitution coefficient in a collision between two spheres. Japanese Journal of Applied Physics, v. 26, p. 1230 - 1233, 1987.

LAN, Y.; ROSATO, A. D. Macroscopic behavior of vibrating beds of smooth inelastic spheres. Physics of Fluids, v. 7, p. 1818 - 1831, 1995.

LAN, Y.; ROSATO, A. D. Convection related phenomena in granular dynamics simulations of vibrated beds. Physics of Fluids, v. 12, p. 3616 - 3624, 1997.

LANKARANI, H. M.; NIKRAVESH, P. E. A contact force model with hysteresis damping for impact analysis of multibody systems. Journal of Mechanical Design, v. 112, p. 369 376, 1990.

LANKARANI, H. M.; NIKRAVESH, P. E. Continuous contact force models for impact analysis in multibody systems. Nonlinear Dynamics, v. 5, p. 193 - 207, 1994.

LIU, J.-F. He's variational approach for nonlinear oscillators with high nonlinearity. Computers $\&$ Mathematics with Applications, v. 58, n. 11-12, p. 2423 - 2426, 2009.

LIU, Y. et al. Modelling of a vibro-impact capsule system. International Journal of Mechanical Sciences, v. 66, p. 2 -11, 2013.

LU, H. et al. Prediction of particle motion in a two-dimensional bubbling fluidized bed using discrete hard-sphere model. Chemical Engineering Science, v. 60, n. 12, p. 3217 $3231,2005$.

LUN, C. K. K.; SAVAGE, S. B. The effects on an impact velocity dependent coefficient of restitution on stresses developed by sheared granular materials. Acta Mechanica, v. 63, n. 1 , p. 15-44, 1986.

MICKENS, R. Comments on the method of harmonic balance. Journal of Sound and Vibration, v. 94, p. 456 - 460, 1984.

MICKENS, R. Oscillations in an $x^{4 / 3}$ potential. Journal of Sound and Vibration, v. 246, n. 2 , p. $375-378,2001$.

MINDLIN, R. Compliance of elastic bodies in contact. ASME Journal of Applied Mechanics, v. 16, p. 259-268, 1949.

MINDLIN, R.; DERESIEWICZ, H. Elastic spheres in contact under varying oblique forces. ASME Journal of Applied Mechanics, v. 20, p. 327-344, 1953.

MOMANI, S. Analytical approximate solutions of nonlinear oscillators by the modified decomposition method. International Journal of Modern Physics C, v. 15, n. 07, p. 967-979, 2004.

MOMANI, S.; ERTüRK, V. S. Solutions of non-linear oscillators by the modified differential transform method. Computers 83 Mathematics with Applications, v. 55, n. 4, p. $833-842,2008$. 
MORAES, F. H. et al. Influence of ideal and non-ideal excitation sources on the dynamics of a nonlinear vibro-impact system. Journal of Theoretical and Applied Mechanics, v. 51, n. 3 , p. $763-774,2013$.

MüLLER, P.; PöSCHEL, T. Oblique impact of frictionless spheres: on the limitations of hard sphere models for granular dynamics. Granular Matter, v. 14, p. 115-120, 2012.

NAVARRO, H. A.; BALThAZAR, J. M.; BRASIL, R. M. L. R. F. Vibrations due to impact in a non ideal mechanical system with a non-linear hertizian contact model. In: ASME. ASME 2014 International Design Engineering Technical Conferences and Computers and Information in Engineering Conference. Buffalo, NY, USA, 2014. (26th Conference on Mechanical Vibration and Noise, DETC2014-34145), p. V008T11A041.

NAVARRO, H. A.; BRAUN, M. P. de S. Determination of the normal spring stiffness coefficient in the linear spring-dashpot contact model of discrete element method. Powder Technology, v. 246, p. $707-722,2013$.

NAVARRO, H. A.; CVETICANIN, L. Amplitude-frequency relationship obtained using hamiltonian approach for oscillators with sum of non-integer order nonlinearities. Applied Mathematics and Computation, v. 291, p. 162-171, 2016.

NAYFEH, A. H. Introduction to Perturbation Techniques. New York, NY, USA: John Wiley \& Sons, 1981.

NAYFEH, A. H. Perturbations Methods. Germany: Wiley-VCH, 2004. (Physics Textbook).

NAYFEH, A. H.; MOOK, D. T. Nonlinear Oscillations. New York, NY: John Wiley and Sons, 1976.

NEWTON, I. The Method of Fluxions and Infinite Series : with its Application to the Geometry of Curve-lines. London: London: Henry Woodfall (1736), 1671.

NORDMARK, A. Non-periodic motion caused by grazing incidence in an impact oscillator. Journal of Sound and Vibration, v. 145, n. 2, p. 279 - 297, 1991.

NOURAZAR, S.; MIRZABEIGY, A. Approximate solution for nonlinear duffing oscillator with damping effect using the modified differential transform method. Scientia Iranica, v. 20, n. 2 , p. $364-368,2013$.

PADé, H. Sur la représentation approchée d'une fonction par des fractions rationnelles. Annales scientifiques de École Normale Supérieure, v. 3 (série), n. 9 (tome), p. 3-93 (supplément), 1892.

PADTHE, A. K. et al. Feedback stabilization of snap-through buckling in a preloaded two-bar linkage with hysteresis. International Journal of Non-Linear Mechanics, v. 43, n. 4 , p. $277-291,2008$.

PILIPCHUK, V. Strongly nonlinear vibrations of damped oscillators with two nonsmooth limits. Journal of Sound and Vibration, v. 302, n. 1-2, p. 398 - 402, 2007.

PILIPCHUK, V. N. Nonlinear Dynamics: Between Linear and Impact Limits. Berlin, Heidelberg: Springer-Verlag, 2010. v. 52. (Lecture Notes in Applied and Computational Mechanics, v. 52). 
PUKHOV, G. E. Computational structure for solving differential equations by taylor transformations. Cybernetics and Systems Analysis, v. 14, n. 383-390, 1978.

PUKHOV, G. E. Expansion fourmulas for differential transforms. Cybernetics and Systems Analysis, v. 17, p. 460-464, 1981.

PUKHOV, G. E. Differential transforms and circuit theory. International Journal of Circuit Theory and Applications, Wiley Subscription Services, Inc., A Wiley Company, v. 10, n. 3, p. 265-276, 1982.

ROSENBERG, R. M. The ateb(h)-functions and their properties. Quarterly of Applied Mathematics, v. 21, n. 1, p. 37-47, 1963.

ROY, R.; OLVER, R. A. A. F. W. J.; WONG, R. Algebraic and analytic methods. In: OLVER, F. W. J. et al. (Ed.). NIST Handbook of Mathematical Functions. New York, NY, USA: National Institute of Standards and Technology, Cambridge University Press, 2010. cap. 1, p. 1-39.

SADEGHZADEH, S.; KABIRI, A. Application of higher order Hamiltonian approach to the nonlinear vibration of micro electro mechanical systems. Latin American Journal of Solids and Structures, scielo, v. 13, n. 3, p. 478 - 497, 2016.

SHäFER, J.; DIPPEL, S.; WOLF, D. E. Force schemes in simulations of granular materials. Journal de Physique I France, v. 6, n. 1, p. 5-20, 1996.

SHAW, S. W.; HOLMES, P. J. A periodically forced piecewise linear oscillator. Journal of Sound and Vibration, v. 90, n. 1, p. 129-155, 1983.

SILBERT, L. E. et al. Granular flow down an inclined plane: Bagnold scaling and rheology. Physical Review E, v. 64, p. 051302, 2001.

SILBERT, L. E.; LANDRY, J. W.; GREST, G. S. Granular flow down a rough inclined plane: transition between thin and thick piles. Physics of Fluids, v. 15, p. 1-10, 2003.

SIMO, J. C.; HUGHES, T. J. R. Computational Inelasticity. New York: Springer-Verlag, 1997.

SOMMERFELD, A. Beitrage zum dynamischen ausbau der festigkeislehre. Z. Ver. Dtsch. Ing., n. 46, p. 391-394, 1902.

SOUZA, S. de et al. Impact dampers for controlling chaos in systems with limited power supply. Journal of Sound and Vibration, v. 279, n. 3-5, p. 955 - 967, 2005.

STEVENS, A. B.; HRENYA, C. M. Comparison of soft-sphere models to measurements of collision properties during normal impacts. Powder Technology, v. 154, n. 2-3, p. $99-$ $109,2005$.

SUN, J.; BATTAGLIA, F.; SUBRAMANIAM, S. Hybrid two-fluid DEM simulation of gas-solid fluidized beds. Journal of Fluids Engineering, v. 129, n. 11, p. 1394-1403, 2007.

SYAMLAL, M. MFIX documentation: Numerical guide. Morgantown, WV, USA, 1998. Disponível em: <https://mfix.netl.doe.gov/documentation/numerics.pdf $>$. 
SYAMLAL, M.; ROGERS, W.; O'BRIEN, T. J. MFIX documentation theory guide. Morgantown, WV, USA, 1993. Disponível em: < https://mfix.netl.doe.gov/documentation/ Theory.pdf $>$.

TIMOSHENKO, S. Vibration Problems in Engineering. Second. New York, NY, USA: D. Van Nostrand Company Inc., 1937.

TIMOSHENKO, S. P.; GOODIER, J. N. Theory of Elasticity. New York: McGraw-Hill, 1970.

TSUJI, Y.; KAWAGUCHI, T.; TANAKA, T. Discrete particle simulation of two-dimensional fluidized bed. Powder Technology, v. 77, n. 1, p. 79 - 87, 1993.

TSUJI, Y.; TANAKA, T.; ISHIDA, T. Lagrangian numerical simulation of plug flow of cohesionless particles in a horizontal pipe. Powder Technology, v. 71, n. 3, p. 239 - 250, 1992.

VENKATARANGAN, S.; RAJALAKSHMI, K. A modification of adomian's solution for nonlinear oscillatory systems. Computers 8 Mathematics with Applications, v. 29, n. 6, p. $67-73,1995$.

WALTON, O. R.; BRAUN, R. L. Viscosity, granular-temperature, and stress calculations for shearing assemblies of inelastic, frictional disks. Journal of Rheology, v. 30, n. 5, p. 949 $-980,1986$.

WANG, T.; HE, Y.; KIM, D. R. Granular temperature and rotational characteristic analysis of a gas-solid bubling fluidized be under different gravities using discrete hard sphere model. Powder Technology, v. 271, p. 35-48, 2015.

WATANABE, T. Forced vibration of continous system with nonlinear boundary conditions. ASME Journal of Mechanical Design, v. 100, p. 487-491, 1978.

WEBER, M. W. Simulation of cohesive particle flows in granular and gas-solid systems. Tese (PhD Thesis) — University of Colorado, Boulder, Colorado, USA, 2004.

XU, L.; HE, J.-H. Determination of limit cycle by Hamiltonian approach for strongly nonlinear oscillators. International Journal of Nonlinear Sciences and Numerical Simulation, v. 11, n. 12, p. 1097-1101, 2010.

YAZDI, M.; TEHRANI, P. Rational variational approaches to strong nonlinear oscillations. International Journal of Applied and Computational Mathematics, Springer, p. 1-15, 2016.

YE, K.; LI, L.; ZHU, H. A note on the Hertz contact model with nonlinear damping for pounding simulation. Earthquake Engineering and Structural Dynamics, v. 38, n. 9, p. 1135-1142, 2009.

YIGIT, A. S.; ULSOY, A. G.; SCOTT, R. A. Spring-dashpot models for the dynamics of a radially rotating beam with impact. Journal of Sound and Vibration, v. 142, n. 3, p. 515 $-525,1990$.

YILDIRIM, A. et al. Analysis of nonlinear oscillations of a punctual charge in the electric field of a charged ring via a Hamiltonian approach and the energy balance method.

Computers and Mathematics with Applications, v. 62, n. 1, p. 486 - 490, 2011. 
YILDIRIM, A.; SAADATNIA, Z.; ASKARI, H. Application of the Hamiltonian approach to nonlinear oscillators with rational and irrational elastic terms. Mathematical and Computer Modelling, v. 54, n. 1-2, p. 697 - 703, 2011.

YILDIRIM, A. et al. Higher order approximate periodic solutions for nonlinear oscillators with the Hamiltonian approach. Applied Mathematics Letters, v. 24, n. 12, p. 2042 - 2051, 2011.

ZHOU, J. K. Differential Transformation and its Applications for Electrical Circuits. Wuhan, China: Huazhong University Press, 1986.

ZHU, H. P. et al. Discrete particle simulation of particulate systems: Theoretical developments. Chemical Engineering Science, v. 62, n. 13, p. 3378 - 3396, 2007.

ZHU, H. P. et al. Discrete particle simulation of particulate systems: A review of major applications and findings. Chemical Engineering Science, v. 63, n. 23, p. 5728 - 5770, 2008.

ZHU, Q.; ISHITOBI, M. Chaos and bifurcations in a nonlinear vehicle model. Journal of Sound and Vibration, v. 275, n. 3-5, p. 1136 - 1146, 2004.

ZUKOVIC, M.; CVETICANIN, L. Chaos in non-ideal mechanical system with clearance. Journal of Vibration and Control, v. 15, n. 8, p. 1229-1246, 2009. 